\title{
Lahcen Darhouani
}

\section{LES TECHNOLOGIES DE L'INFORMATION ET DE LA COMMUNICATION (TIC) COMME MOTEUR DE L'INNOVATION DANS LES CONOMIES TERRITORIALES}

\author{
Thèse présentée \\ à la Faculté des études supérieures de l'Université du Québec à Chicoutimi \\ comme exigence partielle du programme de doctorat \\ en Développement Régional \\ offert coinjointenent à l'Université du Québec à Chicoutimi \\ et à l'Université du Québec à Rimouski \\ pour l'obtention du grade de Philosophiæ doctor ( $\mathrm{Ph} . \mathrm{D}$.
}

\author{
FACULTÉ DES SCIENCES HUMAINES \\ UNIVERSITÉ DU QUÉBEC À CHICOUTIMI \\ CHICOUTIMI
}

Mai 2005

(C) Lahcen Darhouani, 2005 


\section{Bibliothèque}

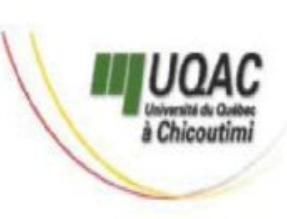

\section{Mise en garde/Advice}

Afin de rendre accessible au plus Motivated by a desire to make the grand nombre le résultat des results of its graduate students' travaux de recherche menés par ses research accessible to all, and in étudiants gradués et dans l'esprit des accordance with the rules règles qui régissent le dépôt et la governing the acceptation and diffusion des mémoires et thèses diffusion of dissertations and produits dans cette Institution, theses in this Institution, the I'Université du Québec à Université du Québec à Chicoutimi (UQAC) est fière de Chicoutimi (UQAC) is proud to rendre accessible une version make a complete version of this complète et gratuite de cette œuvre. work available at no cost to the reader.

L'auteur conserve néanmoins la The author retains ownership of the propriété du droit d'auteur qui copyright of this dissertation or protège ce mémoire ou cette thèse. thesis. Neither the dissertation or Ni le mémoire ou la thèse ni des thesis, nor substantial extracts from extraits substantiels de ceux-ci ne it, may be printed or otherwise peuvent être imprimés ou autrement reproduced without the author's reproduits sans son autorisation. permission. 


\section{Table des matières}

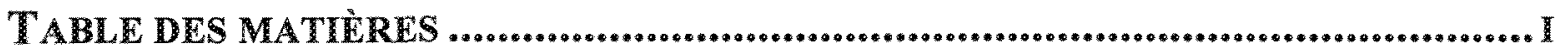

LISTE DES TABLEAUX

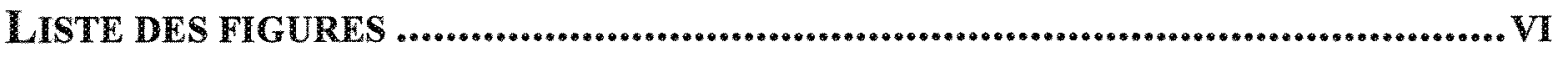

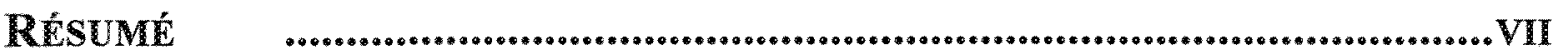

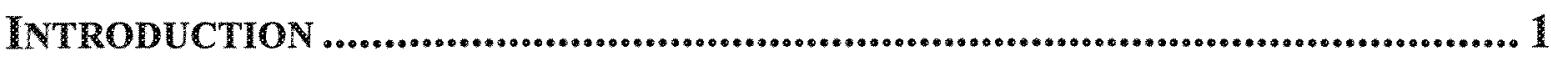

CHAPITRE 1 : L'INFORMATIQUE, LE CHANGEMENT TECHNOLOGIQUE ET LES TIC

1.1. Le chamgement technologique..................................................................... 11

1.1.1. Le changement technologique et l'innovation ..........................................12

1.1.2. L'informatique à la source du changement technologique............................15

1.1.3. L'influence d'Internet .................................................................................21

1.2. Le changement technologique et l'entreprise............................................. 25

1.2.1. Effets des changements technologiques dans le monde des

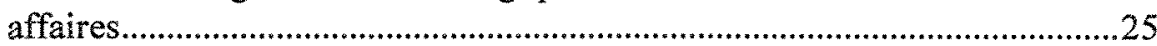

1.2.2. Dimensions économiques d'un changement technologique..............................

1.2.3. Effets des changements technologiques sur la socialisation ...........................31

1.3. Exigences conjoncturelles et TIC.................................................................. 35

1.3.1. L'influence des TIC .................................................................................36

1.3.2. Les TIC accélèrent le temps et suppriment la distance..................................40

1.3.3. Les TIC : entre l'État et le secteur privé.......................................................41

1.4. La conjoncture québécoise ............................................................................. 48

Conclusion ..................................................................................................................... 52

\section{CHAPITRE 2 : L'INFORMATION, SĖVE DES RÉSEAUX D'ENTREPRISES}

2.1. L'information ..................................................................................................... 55

2.1.1. Types d'information.....................................................................................5

2.1.2. Sources et immatérialité de l'information ....................................................56

2.1.3. Rôles et effets de l'information ...................................................................58

2.1.4. L'information comme source de changement..............................................61

2.2. Les réseaux : caractéristiques et avantages ................................................. 62

2.2.1. Réseau ou alliance ................................................................................65

2.2.2. Réseaux et information .............................................................................66

2.2.3. Réseaux et communication..........................................................................69

2.3. Les réseaux d'entreprises et l'apprentissage .............................................. 71

2.3.1. Réseaux d'entreprises et circulation des connaissances ...............................71

2.3.2. L'apprentissage et l'innovation....................................................................76

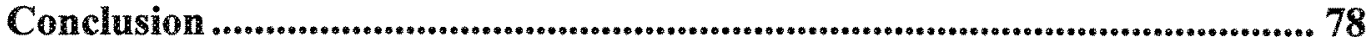


CHAPTRE 3: LES EFFETS DE LA TAILLE DES FIRMES

3.1. Aux origines du débat..................................................................................... 82

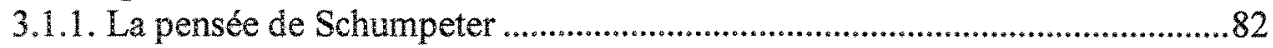

3.1.2. Interprétation et utilité des perspectives de Schumpeter ..............................85

3.2. L'innovation s'accroût avec la taille de la firme ........................................ 87

3.3. Pas de relation entre la taille de l'entreprise et l'innovation ................ 92

3.4. Une relation négative entre la taille de la firme et

l'innovation ................................................................................................... 95

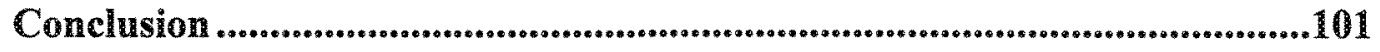

\section{CHAPITRE 4 : L'INFLUENCE DU TERRITORE ET DU MILIEU}

4.1. Milieu innovateur et systèmes d'innovation .............................................105

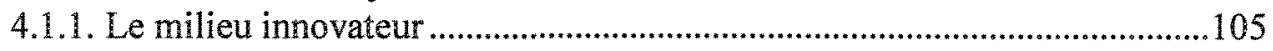

4.1.2. Le système national d'innovation ..............................................................111

4.1.3. Le système régional d'innovation .............................................................116

4.1.4. Le système spatial d'innovation..................................................................119

4.2. Les TIC diversifient les horizons ................................................................120

4.3. De nouvelles donnes conjoncturelles : la proximité et la distance ......................................................................................................124

4.3.1. La proximité, une notion au contour extensible..........................................125

4.3.2. La proximité et l'apprentissage .................................................................130

Conclusion

\section{CHAPITRE 5 : TERRITOIRES ÉTUDIÉS ET OFFRE D'INFORMATION}

5.1. Les territoires étudiés....................................................................................135

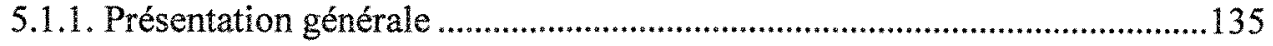

5.1.2. L'activité économique …………………………………………………....138

5.1.3. Caractéristiques particulières ......................................................................141

5.2. Synergies de développement .......................................................................146

5.3. À l'appui de l'entreprise, l'information disponible.................................150

5.3.1. L'information de sources privées..............................................................150

5.3.2. L'information de sources publiques.........................................................153

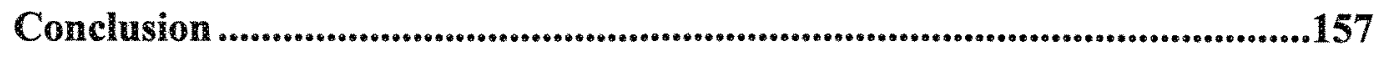

\section{Chapttre 6 : Problématique et orientation de la} RECHERCHE

6.1. Les variables

6.1.1. Les besoins, la demande et la circulation de l'information .......................160

6.1.1.1. Les sources d'information .....................................................161

6.1.1.2. Les moyens d'interaction. 
6.1.1.3. Les types d'information.......................................................162

6.1.1.4. Les activités extérieures........................................................163

6.1.1.5. Les systèmes de communication...........................................163

6.1.1.6. Le classement de l'information .................................................164

6.1.2. L'innovation technologique.................................................................164

6.1.2.1. Le soutien extérieur.................................................................165

6.1.2.2. Le branchement à l'Internet......................................................165

6.1.2.3. L'usage du Web ....................................................................165

6.1.2.4. Le commerce électronique......................................................1 166

6.1.2.5. Le matériel informatique et les logiciels ...............................166

6.2. Les orientations méthodologiques.....................................................168

6.2.1. Position du problème .................................................................................169

6.2.2. La question centrale de la recherche ......................................................170

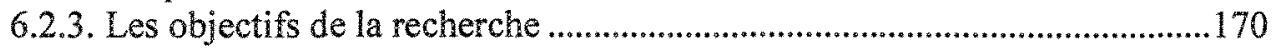

\section{CHAPITRE 7 : LES DONNÉES RECUEILLIES}

7.1. Le profil des entreprises....................................................................................173

7.2. L'information : besoins, demande et circulation......................................177

7. 2.1. Les sources d'informations économiques..................................................177

7. 2.2. Les moyens d'interaction.........................................................................179

7. 2.3. Les types d'information............................................................................180

7. 2.4. Les activités à l'extérieur......................................................................182

7. 2.5. L'affiliation à des réseaux d'entreprises....................................................183

7. 2.6. Les systèmes de communication interne .................................................184

7. 2.7. Le classement de l'information .................................................................185

7.3. L'appropriation de la nouvelle technologie..............................................187

7. 3.1. Le branchement des entreprises à Internet ..............................................187

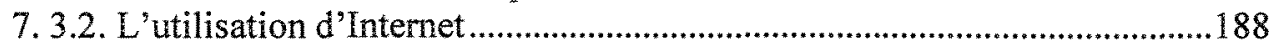

7. 3.3. L'usage du Web et de son potentiel .......................................................190

7. 3.4. Le soutien extérieur................................................................................191

7. 3.5. Le matériel informatique et les logiciels ................................................192

7.4. L'enseignement des données recueillies................................................193

7.4.1. Sources d'information ..............................................................................195

7.4.2. Moyens d'interaction .....................................................................................196

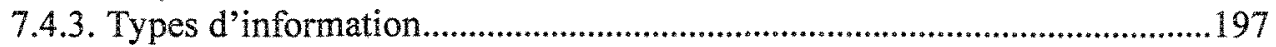

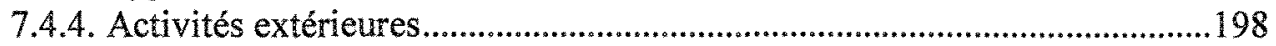

7.4.5. Moyens de communication interne ..........................................................200

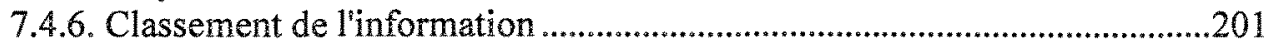

7.4.7. Soutien extérieur ………………………….............................................202

7.4.8. L'usage de l'Internet................................................................................203

7.4.9. L'appropriation du matériel informatique ....................................................204

7.4.10. L'appropriation des logiciels ..................................................................205

Conclusion ..................................................................................................................................211 


\section{CHAPITRE 8 : LEÇONS CONCURRENTES TIRÉES DE L'ANALYSE ET DES OBSERVATIONS SUR LE TERRAIN}

8.1. Le vécu entrepreneurial ..........................................................................213

8.1.1. Le matériel informatique et les logiciels ....................................................213

8.1.2. Les effets de la taille d'une entreprise .......................................................218

8.1.3. Le commerce électronique et l'exploitation du potentiel du

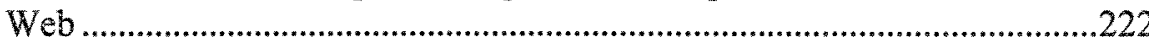

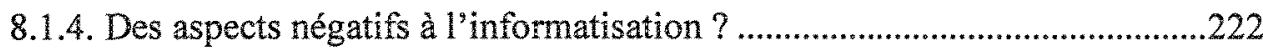

8.1.5. L'entreprise et son milieu ..........................................................................2223

8.1.5.1. Le facteur régional .....................................................................223

8.1.5.2. Les TIC induisent des changements sociaux..........................224

8.2. Perspectives ......................................................................................................225

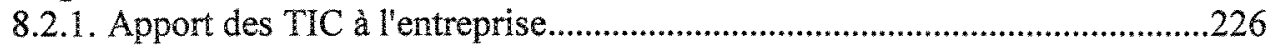

8.2.1.1. Les besoins informationnels..................................................226

8.2.1.2 L'innovation technologique ................................................222

8.2.2. Des possibilités à explorer davantage ...........................................................228

8.2.2.1. Pour l'entreprise : des orientations à considérer ........................228

8.2.2.2. L'appui des pouvoirs publics ...................................................229

8.2.2.3. Horizons technologiques des territoires étudiés........................230

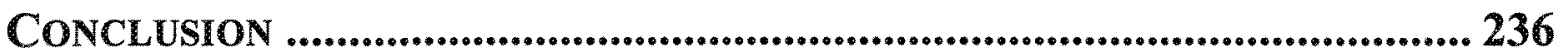

BIBLIOGRAPHIE ..................................................................................... 242 


\section{Liste des tableaux}

Tableau 1: La région, cadre spatial pour l'étude de l'innovation ........................................................116

Tableau 2: Obstacles au développement dans divers types de système régional d'innovation ............117

Tableau 3: Les MRC étudiées : population et superficie ........................................................................ 136

Tableau 4: Variation du taux de l'emploi des 15-64 ans et variation du taux de chômage, les quatre

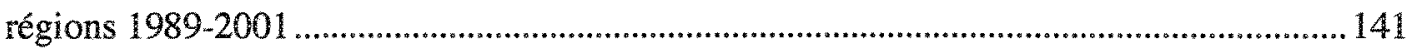

Tableau 5: Population de 15 ans et plus selon la participation au marché du travail, les quatre régions concernées, 2001

Tableau 6: Population de 15 ans ế plus selon la participation au marché du travail, les quatre MRC étudiées, 2001

Tableau 7: Population active de 15 ans et plus selon les industries les plus importantes, quatre régions concernées, 2001

Tableau 8: Population active de 15 ans et plus selon les industries les plus importantes, les quatre MRC étudiées, 2001 144

Tableau 9: Les entreprises de l'échantillon selon le secteur d'activité, (en \%) ....................................174

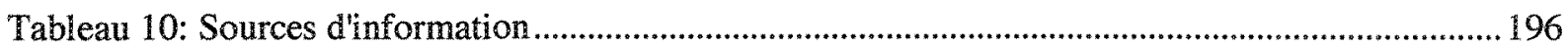

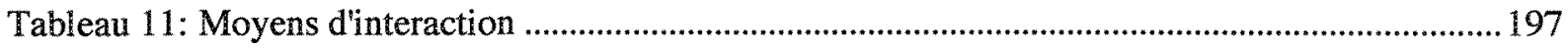

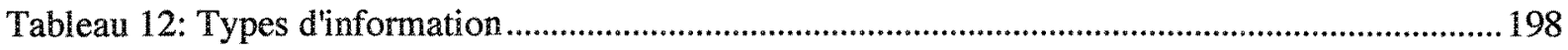

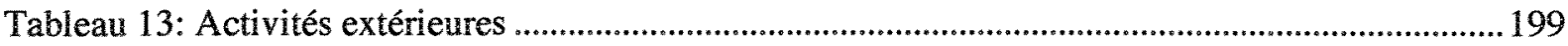

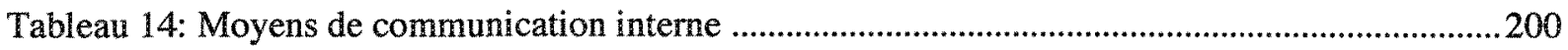

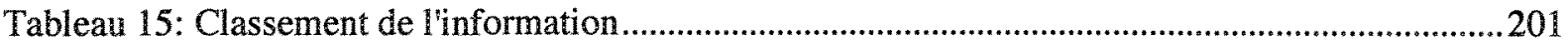

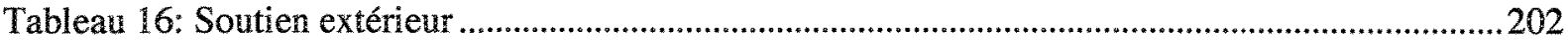

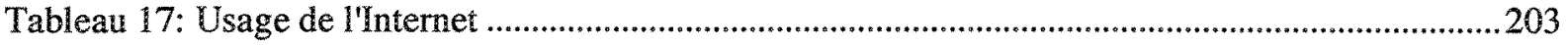

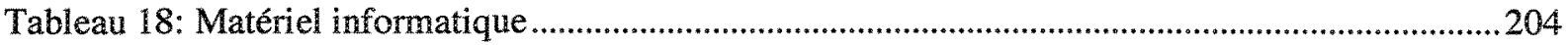

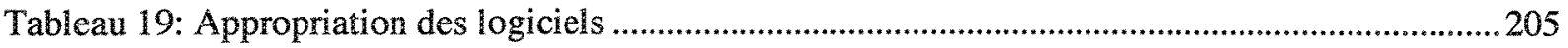




\section{Liste des figures}

Figure 1: Le modèle de Schumpeter 86

Figure 2: Répartition des entreprises de l'échantillon selon leur territoire d'appartenance, (en \%) ... 173

Figure 3: Répartition des entreprises selon le nombre de places d'affaires, en $\%$............................... 175

Figure 4: Répartition des entreprises selon le nombre d'employés, en \%........................................... 175

Figure 5 : Répartition des entreprises selon le nombre d'années d'opération, ...................................... 176

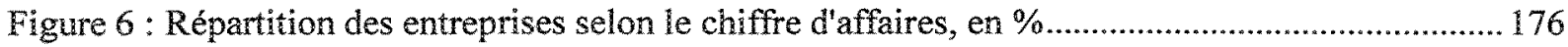

Figure 7: Répartition des entreprises selon les sources de concurrence, en $\% \ldots \ldots \ldots \ldots \ldots \ldots \ldots \ldots \ldots \ldots \ldots \ldots . . . . . . . . .177$

Figure 8: L'affiliation des entreprises à des réseaux ............................................................................... 184

Figure 9: Les systèmes de communication adoptés par les entreprises, tout l'échantillon .................. 185

Figure 10 : Le classement de l'information, tout l'échantillon, en \% ...................................................186

Figure 11: Le branchement des entreprises à l'Internet.......................................................................188

Figure 12: L'usage de l'Internet, tout l'échantillon, en \%............................................................... 189

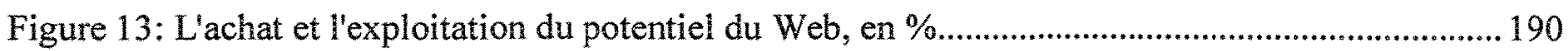

Figure 14: Le soutien extérieur recherché par les entreprises, en \% .................................................191

Figure 15: Comparaison des quatre territoires de l'étude à partir d'un indice global : Sources

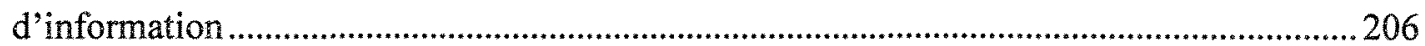

Figure 16: Comparaison des quatre territoires : Moyens d'interaction ................................................207

Figure 17: Comparaison des quatre territoires: Types d'information...................................................207

Figure 18: Comparaison des quatre territoires: Activités extérieures ................................................208

Figure 19: Comparaison des quatre territoires: Moyens de communication interne ...........................208

Figure 20: Comparaison des quatre territoires: Classement de l'information .....................................209

Figure 21: Comparaison des quatre territoires: Soutien extérieur......................................................209

Figure 22: Comparaison des quatre territoires: L'usage de I'Internet...................................................210

Figure 23: Comparaison des quatre territoires: Appropriation du materiel informatique et des logiciels 


\section{Résumé}

Dans un espace géographique donné, le développement inégal des régions est la résultante de plusieurs facteurs qui ont historiquement désavantagé les régions périphériques par rapport aux pôles de croissance métropolitains. Cette situation n'est pourtant pas inéluctable. L'avènement des technologies de l'information et de la communication (TIC) a produit une nouvelle donne, où sont réinventés les modèles d'affaires et disparaissent les barrières spatiotemporelles existant entre fournisseurs d'information et de biens, distributeurs et consommateurs.

La présente étude, réalisée dans quatre municipalités régionales de comté (MRC) du Québec, montre que les dirigeants d'entreprises y sont conscients de la puissance des TIC; qu'en les intégrant de mieux en mieux dans la conduite de leurs opérations, ils protègent l'avenir de leur organisation et concourent au renforcement du dynamisme économique et social de leur territoire. L'appropriation et l'usage des outils informatiques représentent une contribution essentielle au nécessaire rattrapage des territoires périphériques et à l'atteinte d'un développement satisfaisant.

Par :

Lahcen arhouani

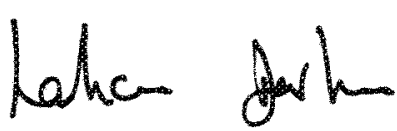

$429.05-05$.

Directeur de la thèse :

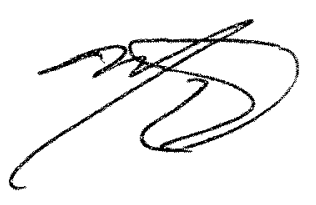




\section{INTRODUCTION}

L'activité économique, de même que l'innovation et tout autre phénomène lié à la nouvelle économie, est rarement répartie de façon égale entre territoires ou même dans un territoire donné. Il y a des raisons à cette inégalité et des conséquences, que les scientifiques tentent de cerner, afin d'aider les décideurs dans l'aménagement d'un développement régional plus cohérent. C'est que la répartition et la localisation de l'activité économique - et partant de l'activité sociale - répondent au jeu d'influences diverses, parmi lesquelles comptent pour beaucoup les éléments caractéristiques d'un territoire. Selon Courlet (2001), le territoire est une notion complexe, qui a de multiples facettes. C'est un acteur qui a sa propre logique d'évolution. Il émane d'une logique collective, ancrée dans un contexte largement dominé par un ensemble d'institutions sociales, qui produisent des normes et génèrent des valeurs. Mais avant tout un territoire est un espace où les acteurs sont appelés à coopérer, à vivre ensemble. L'ensemble des éléments propres à un territoire : histoire, géographie, organisation interne, institutions locales, liens avec d'autres organisations à l'échelon régional, national et international, constitue et particularise ce qu'on peut appeler un système territorial.

Naturellement, le système territorial, ainsi que les autres facteurs de nature économique ou socioculturelle, ont une importance considérable, voire déterminante, dans la décision d'implanter une entreprise dans un territoire, avec les répercussions qui s'ensuivent sur la vie économique et sociale. La prise en considération de ces influences agit sur les pratiques des acteurs de développement par rapport aux ressources disponibles et précise les moyens dont ils disposent au moment d'affronter les incertitudes inhérentes à leur champ d'action. 
Les dynamiques propres à un système territorial déterminent ses chances d'évoluer en un système d'innovation. On réfère par là à la capacité des acteurs d'un territoire à en promouvoir les éléments favorables à la réalisation d'activités propres à valoriser les productions locales. Quand il s'agit d'un territoire dont le développement a pris du retard c'est souvent le cas des territoires périphériques - les acteurs peuvent avoir à faire des efforts supplémentaires d'imagination, d'appropriation technologique et parfois sauter des étapes. L'arrivée massive des technologies d'information et de communication (TIC) stimule les dynamiques économiques et sociales de développement d'un territoire : partout, «les TIC et le commerce électronique bénéficient à une large gamme de processus économiques. Au niveau de l'entreprise, les TIC et leurs application améliorent et accélèrent la communication et permettent une gestion plus efficace des ressources de l'entreprise [...] Au niveau interentreprises, l'Internet et le commerce électronique ont le potentiel de diminuer les cô̂ts des transactions tout en les accélérant et en les rendant plus fiables» (OCDE $2004: 12$ ).

Le territoire n'est pas qu'un espace, une plate-forme neutre, support d'activités diverses. Il est fait des composantes - certaines favorables, d'autres moins - dont l'acteur doit tenir compte en cherchant à profiter des unes et à pallier les autres. Espace géographique où les entreprises évoluent dans un cadre commun, le territoire participe en un sens à l'articulation de l'action économique et sociale. Il est par conséquent dans l'intérêt bien compris des acteurs de coordonner leurs actions et de coopérer pour affronter collectivement les imprévus. Une solidarité inter-organisationnelle peut dès lors s'établir, l'intensité des rivalités diminuer et la concertation progresser, créant un contexte avantageux pour toutes les entreprises.

Solidarité, concertation, des valeurs qui ne se concrétisent pas sans la circulation continue d'un fluide entre les acteurs : l'information et sa communication. En rapport avec l'origine étymologique, on peut voir la communication comme le lien qui s'établit entre correspondants qui échangent de l'information par l'intermédiaire d'un moyen de transmission (Laramée 2001). Dans le cas ici considéré, l'informatique est à la fois le véhicule de l'information, le support de la communication et le principe de la société dite de l'information. C'est la société où domine l'information comme communication d'un savoir de la société informationnelle : une forme particulière d'organisation sociale dans laquelle la création, le traitement et la transmission de l'information deviennent les sources premières de la productivité et du pouvoir (Castells 1998: 42). La productivité est «définie habituellement [...] comme le 
rapport, en volume, d'une production sur un ou plusieurs facteurs de production» (OCDE $2001: 11$ ).

Outre les capitaux, l'appropriation par l'entreprise d'une nouvelle technologie - de conception, fabrication ou gestion - dépend de l'effort préalable d'information puis de décisions éclairées d'investissement. À cet égard il y a des régions gagnantes et d'autres qui le sont moins. Pour les régions comme pour les entreprises, l'avantage vient de ce que, par suite de forts investissements dans les technologies, elles connaissent d'importants gains de productivité, de croissance et d'efficacité. Ce sont les régions gagnantes (Benko et Lipiez 1995). Les régions perdantes seraient celles où l'on n'a pas ou pu améliorer la technologie ou se l'approprier.

Lorsque choisie de façon à bien correspondre aux objectifs de l'entreprise, une nouvelle technologie apporte avec elle efficacité, efficience et capacité concurrentielle accrue :

technology is a central ingredient in economic development. It is behind the proliferation of products and of weapons. It permits entrepreneurs to get an edge on competitors, and it allows one region to be more prosperous than another. Much of what we call technological change is the process of learning by people and, through them, by organizations and nations (Malecki 1991: xi).

Si l'appropriation d'une technologie impose nécessairement un effort de la part des individus et des adaptations dans les entreprises, ses retombées s'étendent au-delà et provoquent éventuellement des changements dans les institutions et organisations de leur environnement: «les stratégies mises en oeuvre par les entreprises en faveur des TIC et du commerce électronique ont des retombées globalement positives sur la performance de l'entreprise [...] L'utilisation des TIC peut contribuer à optimiser la performance des entreprises, en termes de part de marché, de gamme de produits, d'individualisation de l'offre et de réponse à la demande» (OCDE $2004: 14$ ). À terme, l'adoption suffisamment généralisée des technologies de pointe devient, pour un territoire, un puissant levier de progrès économique et de renouveau social (OCDE 2001). C'est ce genre de dynamique qui, souvent, creuse l'écart entre les entreprises et entre les régions. 
En somme, tout indique que nous sommes définitivement entrés dans l'ère numérique; que les applications de la numérisation, notamment les TIC, seront de plus en plus gages de progrès et de prospérité pour les entreprises et ultimement pour les sociétés. On note toutefois une appropriation fort inégale des technologies de pointe, tant chez les individus que dans l'entreprise, ce qui présente une problématique de nature à stimuler l'intérêt des chercheurs. En fait, on ignore comment les rapports à l'informatisation et aux TIC conditionnent cette inégalité, et l'influence que peut avoir cet écart sur certaines contraintes territoriales telles que la distance, la disponibilité des ressources, l'absence d'une économie d'échelle, la densité démographique, etc. Il y a donc là une vaste zone grise autour de l'informatisation et des TIC, de leur appropriation, de leur influence, et c'est vis-à-vis de cette incertitude que s'articulent les débats entre spécialistes depuis quelque temps.

Dans les décennies récentes, les chercheurs ont utilisé différentes approches, diversifié leurs horizons, pour tenter d'expliquer l'évolution des systèmes socio-économiques, soit du point de vue macro ou microéconomique. L'informatisation récente et ses applications croissantes dans l'activité humaine, tout en facilitant les tâches pour l'utilisateur, ne les a pas simplifiées pour le chercheur. Pour le spécialiste des sciences régionales, le rôle des TIC dans les territoires, et dans les entreprises qui s'y trouvent, est devenu une question centrale: comment et à quel degré on se les approprie, ce qu'on en fait à l'interne et à l'externe, et les conséquences qui en résultent pour le développement. Tel est le thème directeur de la présente recherche.

Si la science régionale se construit «une représentation du monde, au sens de représentation mentale abstraite qui prend son sens dans le cadre d'idéologies et de problématiques» (Bailly 1992 : 4), il fallait que les choix conceptuels et méthodologiques adoptés pour cette étude s'exercent concrètement dans un cadre spatial représentatif. En effet, le choix d'un cadre spatial approprié est important. Il détermine jusqu'à un certain point la nature des problématiques étudiées et les dimensions de l'analyse :

suivant les échelles privilégiées apparaissent des arrangements spatiaux et des pratiques différentes. [...] Les différences d'échelle produisent fréquemment des différences de nature des problèmes étudiés ; du niveau scalaire dépend la finesse de l'analyse (Bailly 1992: 4). 
Pour les fins de cette recherche, l'unité spatiale choisie est le territoire des municipalités régionales de comté, (MRC). Par leurs dimensions, par leurs caractéristiques, les MRC s'imposaient comme un choix logique.

Une MRC est constituée du groupement des municipalités locales situées dans un territoire donné; les MRC constituent au Québec des entités territoriales bien différenciées, rurales et urbaines, et de tailles relativement importantes. Ces territoires ont une vocation supra-locale, qui leur donne un statut intermédiaire entre le niveau local, étroitement lié à la vie quotidienne, et les paliers supérieurs, régionaux puis national. La taille intermédiaire de la MRC, entre l'échelon local et les échelons supérieurs, devait permettre de circonscrire l'étude et de mesurer la capacité des entreprises à s'approprier convenablement les TIC. Dans le cadre de cette étude, notre choix s'est porté sur quatre territoires MRC, dont les caractéristiques en font des champs de recherche raisonnablement représentatifs.

Deux points de vue, complémentaires, orientent notre démarche. D'une part, l'approche en terme d'économie d'information ouvre des perspectives intéressantes, autant théoriques qu'empiriques. Elle reflète une dynamique économique et sociale fortement influencée par l'information:

on utilise la notion d'économie d'information pour souligner qu'une des caractéristiques majeures des économies développées contemporaines tient à l'ampleur et à la diversité des usages qu'elles font de l'information sous toutes ses formes et à tous les niveaux. [...]. Dans une acception plus complète, cette économie de l'information prend acte aussi de la montée des connaissances et du rôle accru des savoirs qui semblent aller de pair avec ces évolutions technologiques (Petit $1998: 16$ ).

D'autre part, cette recherche s'intéresse principalement à la pénétration des technologies d'information et de communication (TIC) dans l'activité économique, tout en n'oubliant pas que ces mêmes technologies pénètrent aussi la vie sociale. Et que dans les deux cas il y a des répercussions sur le développement.

Des repères nouveaux orientent les activités économiques, accompagnés d'une accélération des processus liés à la production et à la consommation, «as an outcome of the manner in which technology is generated, acquired and utilized» (Malecki 1991 :113). Ces repères sont 
souvent déterminés par les enjeux conséquents à une économie dite d'information. Ils sont influencés par les pratiques des entreprises; pratiques qui s'insèrent dans un cadre multiorganisationnel, influencent les processus de prise de décision et déterminent la place qu'occupent ces entreprises au sein des systèmes en place. Plusieurs facteurs liés au cadre commun et à la place de l'entreprise dans un système multi-organisationnel influent sur sa productivité et sa capacité concurrentielle. Ils contribuent aussi puissamment à définir les stratégies qu'adopte l'entreprise pour affronter ses problèmes de production et les contraintes du marché. Au regard des possibilités d'innovation et de progrès qu'offrent l'évolution informatique et les TIC, notre étude considère les entreprises sous quatre aspects principaux: l'appropriation de linformatique, la taille des entreprises, l'utilisation du Web et les réseaux d'entreprises.

Sous-jacent à cette recherche se trouve un questionnement, à savoir : pourquoi voit-on dans certains territoires l'innovation fleurir, et se faire rare dans d'autres? Comment les technologies contribuent-elles à faire la différence? S'il est vrai que les systèmes qui permettent la communication, l'organisation et le traitement de l'information sont vitaux pour l'entreprise, il y a lieu de bien voir les choix que font les dirigeants d'entreprises, et surtout les raisons à la base de ces choix, quant à l'utilisation des moyens classiques ou des nouveaux systèmes. Cet exercice s'avère utile pour comprendre comment les acteurs économiques et les territoires intègrent les anciens et les nouveaux systèmes informationnels et laisse pressentir l'influence avantageuse des TIC pour le développement.

Le présent travail comprend huit chapitres. Le premier est consacré aux changements technologiques. Il s'agit d'abord d'explorer les questions que soulèvent le changement technologique et l'innovation. L'accent est mis sur les pratiques entrepreneuriales et les technologies nouvelles, considérées à la source des changements en cours. Il s'agit de voir comment ces changements ont provoqué des transformations profondes dans des contextes variés. L'avènement de l'Internet, de même que les exigences conjoncturelles, ont accéléré ces transformations. En fait, l'arrivée massive des TIC a modifié les rapports au temps et à la distance. Pour cette raison, l'État et le secteur privé ont accordé une importance particulière aux composantes technologiques, surtout celles qui ont des effets marqués sur le monde des affaires en général, et sur l'înnovation en particulier. 
S'il est nécessaire pour l'entreprise de se tenir techniquement à jour, il lui est aussi indispensable de disposer de toute l'information pertinente à son fonctionnement. $\mathrm{A}$ cet égard il y a lieu de considérer l'apport du réseautage. C'est l'objet du $2^{\mathrm{e}}$ chapitre. La littérature relative à ces questions indique que bien souvent c'est au sein des réseaux d'entreprises que s'établissent de nouvelles formes de solidarité, de coopération et de concertation entre les acteurs d'un territoire. Lieux d'interaction, où circulent les connaissances et l'information stratégique, les réseaux sont à juste titre considérés comme des lieux d'apprentissage collectif et comme tremplins de l'innovation.

L'influence de la taille des entreprises sur l'innovation a donné lieu chez les chercheurs à des vues contradictoires, que résume le troisième chapitre. Aux origines du débat, les perspectives de Schumpeter, à première vue paradoxales, sont à cet égard incontournables, car elles ont fourni des idées permettant de mieux comprendre les comportements d'entreprises par rapport à l'innovation, et montré que le contexte où se déroule une étude à ce sujet influe nécessairement sur les conclusions. La littérature plus récente relative à ces questions est par la suite passée en revue.

Au quatrième chapitre sont abordées des notions importantes pour le spécialiste des sciences régionales : le territoire, le milieu et les rapports qu'ils entretiennent avec l'innovation. Une première section analyse les écrits ayant traité de milieu innovateur et de systèmes d'innovation. L'examen de la littérature spécialisée permet de dégager des éléments forts et certaines lacunes relevant de l'usage de notions comme le milieu innovateur et les systèmes régional, national et spatial d'innovation. Par son importance théorique et les conclusions pratiques qu'il entraine, le recours à ces notions aide à comprendre les dynamiques de nos sociétés. En fait, l'usage croissant des TIC semble avoir quelque peu brouillé les pistes, car il a changé les représentations que les acteurs se font de la proximité et de la distance; cela ressort d'une partie de la littérature spécialisée. Ce nouveau regard fait prendre conscience d'une possibilité inestimable, offerte par la proximité, réelle ou virtuelle: loccasion d'apprendre collectivement.

L'examen des questions théoriques et des concepts qui constituent la plateforme de cette recherche était un préalable au cinquième chapitre, qui décrit et tente de saisir le dynamisme économique et social des quatre territoires retenus pour cette étude : la Communauté Urbaine 
de Québec, Saguenay, Rivière-du-Loup et Manicouagan. L'intérêt porte principalement sur les activités économiques et le contexte social : cela a semblé important pour comprendre et comparer les synergies respectives de développement. Le rôle de l'information quant à ces synergies est ensuite précisé. Une attention particulière est donc accordée aux sources privées et publiques d'information.

Le cadre adopté pour cette recherche amenait à définir, au sixième chapitre, les variables qui devraient ensuite être mesurées sur le terrain. Définies en fonction de notre problématiq̨ue de départ et des objectifs poursuivis, ces variables concernent trois axes principaux: 1 . l'appropriation du matériel informatique et des logiciels, et leur usage dans les entreprises; 2. les besoins, la demande et la circulation de l'information. Il s'agit des sources d'informations économiques, des moyens d'interaction et des types d'information. De même, l'intérêt porte sur les systèmes de communication interne et sur le classement de l'information; 3 . 11 est enfin question des réseaux d'entreprises et des activités sociales et d'appropriation du matériel informatique et des logiciels. Ces variables ont conduit à formuler notre hypothèse relative aux axes de recherches précités

Le chapitre sept présente les données cueillies au cours de l'enquête et s'ouvre sur une description du profil général des entreprises. L'attention se porte ensuite sur l'exploitation de l'information : besoins, demande, sources et circulation de l'information. La section suivante traite de l'innovation technologique dans l'entreprise, particulièrement les TIC. L'interprétation des résultats de l'enquête occupe la dernière section de ce chapitre. Notre méthode d'analyse est d'abord décrite. C'est à l'aide des tableaux dynamiques croisés obtenus et des graphiques que nous avons comparé les quatre territoires étudiés.

Le huitième et dernier chapitre présente les perspectives que l'on peu dégager à la fois des données recueillies et de ce que nous avons observé sur le terrain. Les idées qui y sont présentées résument les leçons tirées des discussions que nous avons eues à de multiples occasions avec les principaux acteurs de développement et avec une centaine de dirigeants d'entreprises. Particulièrement pour les PME implantées en territoires périphériques, des perspectives intéressantes se dégagent de la présente recherche, et aussi quelques enseignements. On peut y constater que l'appropriation du matériel informatique et des logiciels crée des dynamiques nouvelles de développement. L'usage des TIC a des effets 
avantageux, que n'empêchent ni les incommodités d'un territoire, ni la taille plus ou moins grande des entreprises qui y sont implantées. Ces effets, observables partout, suggèrent qu'il est du plus haut intérêt, pour les entreprises québécoises, de s'engager résolument dans une appropriation croissante des technologies prometteuses de succès. Il apparait de plus que les entreprises auraient avantage à diversifier leurs sources d'information et que les pouvoirs publics devraient s'impliquer de façon plus soutenue dans le développement régional.

Enfin, une conclusion générale sert à récapituler les principales étapes de cette recherche et à en présenter les constatations les plus saillantes. 


\section{CHAPITRE 1}

\section{L'INFORMATIQUE, LE CHANGEMENT TECHNOLOGIQUE ET LES TIC}

Une lecture attentive de la littérature spécialisée permet de reconnaître les améliorations d'ordre technique et, encore davantage les avancées technologiques, qui ont historiquement constitué des changements aux répercussions considérables. Divers aspects du changement se présentent à la réflexion : les conditions de son apparition, de son acceptation, et son influence sur l'économie et la société où il survient. De nos jours le changement s'est fortement accéléré et plusieurs chercheurs $\mathrm{y}$ ont vu un phénomène singulier qu'il convient d'étudier. Ordinairement, le changement assure la continuité d'un développement dont les grands axes existaient préalablement. Mais à l'évidence les transformations actuelles sont d'un tout autre ordre par leur magnitude, leur célérité et leur complexité. La nouveauté semble se manifester non seulement dans le déferlement d'objets issus d'une technologie de pointe, mais surtout dans l'évolution des pratiques quotidiennes associées à l'usage de ces objets.

Il y a changement technologique quand l'usage des objets issus d'une technologie novatrice se généralise, engendre des pratiques nouvelles dans la vie quotidienne, des initiatives dénotant l'entrée des acteurs dans le nouvel ordre économique et social; mais un usage qui, parfois, est aussi à l'origine de difficultés et de crises pour certains acteurs incapables de suivre le rythme. 
Ces manifestations du changement technologique n'ont rien d'accidentel; elles découlent de la complexité même des rapports que les acteurs entretiennent avec l'univers technologique. Elles reflètent également une tendance, avantageuse pour certains acteurs et certains territoires, alors que surviennent ailleurs des crises récurrentes qui ébranlent l'économie et la société.

Par rapport à ces nouvelles réalités, le changement technologique semble avoir des conséquences qui oscillent entre deux tendances diamétralement opposées. L'une affirme que l'adoption de technologies nouvelles stimule le dynamisme des entreprises et des territoires, incite les acteurs à en exploiter le potentiel et en définitive favorise la prospérité; les publications de l'OCDE vont dans ce sens, car elles avancent que «le changement technologique est le moteur de la croissance économique à long terme, et du relèvement du niveau de vie» (OCDE 2001: 119). L'autre tendance remet en question les avantages du nouvel ordre technologique, le considérant responsable de problèmes économiques et sociaux tels que l'instabilité, la perte d'emplois, etc.

Il parait donc indiqué d'examiner, à la lumière de la littérature pertinente, comment les changements technologiques s'imbriquent dans les pratiques existantes et conditionnent les dynamiques économiques et sociales. Une première section porte sur la distinction entre changement technologique et innovation. Suit un examen de la présence de plus en plus ubiquitaire de l'informatique, de son influence grandissante dans l'activité économique et la vie sociale. Une dernière section considère la nouvelle conjoncture provoquée par les technologies de l'information et de la communication (TIC), particulièrement Internet, et les rôles respectifs qui y sont impartis à l'État et au secteur privé.

\subsection{Le chamgement technologique}

Le rôle des technologies, pour le développement dans les milieux, les territoires, attire à bon droit l'attention des chercheurs. La présente section montre l'importance des changements technologiques en cours et les effets de ces changements sur le développement des économies et des sociétés. Les rapports existant entre ces changements et l'innovation sont passés en revue, puis l'influence de l'informatique comme source de ces changements. Il est ensuite question de l'avancée technologique majeure que représente le réseau Internet. 


\subsubsection{Le changement technologique ef linnovation}

Dans la plupart des pays, il existe une collaboration suivie entre l'État, luniversité et l'entreprise. Les gouvernements mettent sur pied divers programmes pour activer la R\&D : subventions à la recherche universitaire, avantages fiscaux aux entreprises, etc. Naturellement, cet effort stimule le changement technologique qu'on peut dès lors considérer comme la résultante d'initiatives variées devant permettre aux acteurs de développement, individuels ou collectifs, de s'adapter aux exigences de la conjoncture du moment. Un changement technologique renvoie en général à deux dimensions différentes, précisées par l'OCDE. "Par technologie, on entend "les moyens connus au moment considéré pour transformer des ressources en produits réclamés par l'économie"'» (OCDE 2001: 11). De plus, on doit faire une distinction entre

la technologie corporelle et incorporelle. Les évolutions de la technologie corporelle sont les avancées dans la conception et la qualité de nouvelles générations de capital et de produits intermédiaires: les machines et les équipements incarnent les fruits de la recherche réalisée par la branche qui produit les biens d'investissements, et d'autres accèdent aux résultats de ces recherches en achetant des biens d'équipement ou des biens intermédiaires nouveaux. La variation de la technologie incorporelle, en revanche, a trait aux avancées de la science, aux plans techniques et aux formules ainsi qu'à la diffusion de la connaissance sur la manière de faire les choses, y compris l'amélioration de la gestion et les changements organisationnels. Cette distinction est importante, parce que la diffusion de l'évolution technique corporelle est tributaire de transactions sur le marché : les investissements en biens de capital ou en produits intermédiaires améliorés se poursuivront jusqu'à ce que leur contribution marginale à la création de revenus devienne égale à leur coût d'utilisation, lui-même fonction du prix du bien d'équipement sur le marché. En revanche, la diffusion de la technique incorporelle ne suppose pas forcément de transactions sur le marché : l'information peut circuler librement, et son utilisation par une personne ne restreint normalement pas la capacité des autres personnes à l'utiliser aussi (OCDE 2001 : 119).

Il est donc important de prendre en compte cette distinction entre l'évolution technique corporelle et incorporelle. Cette distinction montre la complexité de tout changement technologique et de ses effets sur la vie quotidienne. Elle permet aussi de comprendre comment un changement technologique résulte d'une démarche réfléchie par laquelle se localisent dans un certain espace géographique les possibilités de progrès qu'apporte l'innovation. 
Comme le changement technologique, l'innovation prend des formes diverses et les changements qu'elle provoque amplifient l'influence des acteurs sur les dynamiques économiques et sociales de développement. Dans sa Théorie de l'évolution, Schumpeter a identifié cinq cas d'innovation : 1. La fabrication d'un produit nouveau; 2. L'introduction d'une méthode de production nouvelle; 3. L'ouverture d'un nouveau débouché ; 4. La conquête d'une nouvelle source de matières premières; 5 . La mise en oeuvre d'une nouvelle méthode d'organisation de production (Schumpeter $1935: 93$ ).

Cette distinction est intéressante car elle permet de voir comment une innovation sert, dans toutes ses étapes et quelles que soient sa nature et sa dimension, non seulement à enrichir le cadre général qui la produit, mais dans bien des cas à le dépasser. Toute innovation a également des effets profonds, mais qui varient selon le contexte général et surtout selon sa capacité à surmonter les obstacles à la productivité et à la croissance. Schumpeter précise que l'innovation rend plus avantageux le recours aux nouveaux produits ef aux nouvelles méthodes qui

ne concurrencent pas les anciens produits et les anciennes méthodes sur un pied d'égalité, mais avec une supériorité décisive qui peut signer larrêt de mort de ces derniers. Tel est le processus par lequel le progrès pénètre dans une société capitaliste. Pour échapper au risque d'être battue sur ses prix, toute entreprise est finalement obligée de suivre les pionniers, de procéder à son tour à des investissements et, aux fins d'être en mesure de le faire, de remettre en jeu une fraction de ses profits, c'est-à-dire d'accumuler. En conséquence, tout le monde accumule (Schumpeter 1947:53).

Le développement d'une innovation, comme sa généralisation, renvoie à l'un des plus importants mécanismes de la vie économique et sociale, celui de la créativité et de la richesse qui résultent d'interactions diverses, tant individuelles que collectives. On affirme ainsi que toute innovation repose d'abord sur ce qu'ont cré les devanciers et reflète l'effort individuel et collectif visant à dépasser les limites de la vie quotidienne, à faciliter le travail, fabriquer à moindre coût de meilleurs produits, etc. Schumpeter décrit bien l'utilité inappréciable de l'innovation:

Le plus petit acte qu'accomplit quotidiennement un homme, implique un travail intellectuel quantitativement immense : non seulement il faudrait que chaque écolier et chaque maitre de cet enfant soit un géant de l'esprit dépassant toute 
mesure humaine, sil créait pour soi par un acte individuel, conscient, systématique ce quili sait et ce quil utilise; mais il faudrait encore que chaque homme soit un géant par son intelligence pénétrante des conditions de la vie sociale et par sa volonté, pour traverser seulement sa vie quotidienne, sil lui fallait chaque fois acquérir par un travail intellectuel les petits actes dont elle est faite, et leur donner une forme dans un acte créateur. Ceci ne vaut pas seulement pour la connaissance et l'activité dans les limites des fonctions générales de la vie individuelle et sociale, et pour les principes qui, relevant de la pensée, du cceur, de l'action, dominent cette activité, et sont les fruits d'efforts millénaires. Ceci vaut encore pour les produits de temps plus courts et d'une nature spéciale, qui permettent l'accomplissement des devoirs de la vie professionnelle. Précisément les choses, dont l'exécution exigerait d'après ce qui précède, un travail d'une puissance immense, ne demandent aucun travail individuel particulier; elles qui devraient être spécialement difficiles sont en réalité faciles ; ce qui demanderait une capacité surhumaine, est accessible sans défaillance frappante aux moins doués pourvu qu'ils aient un esprit droit (Schumpeter 1935 : 343-344).

Si ce passage montre que l'innovation se bâtit sur des acquis, elle résulte aussi, la plupart du temps, d'un travail assidu et patient. Car elle ne se fonde pas uniquement sur un désir de nouveauté, mais répond ordinairement à des préoccupations concrètes. Par ailleurs les conséquences de l'innovation ne sont pas toujours immédiatement apparentes. C'est pourquoi il importe de considérer son instauration à la lumière de disciplines telles que l'histoire et la sociologie, qui offrent une perspective apte à guider l'action et à l'harmoniser avec les exigences conjoncturelles.

Il arrive qu'une innovation, globalement bénéfique, soit mal reçue, par référence indue à des critères sociohistoriques, ou par peur du changement. Dans la vie sociale comme dans l'activité économique, des acteurs résistent et vont jusqu'à rejeter l'innovation. Comme l'avait constaté Schumpeter, ces comportements ont souvent leur source dans «les groupes menacés par la nouveauté, puis dans la difficulté à trouver la coopération nécessaire de la part des gens dont on a besoin, enfin dans la difficulté à amener les consommateurs à suivre» (Schumpeter $1935: 348$ ). Le développement résulte de cette dualité entre l'innovation et la stagnation, entre l'acceptation de la nouveauté et son rejet. Évidemment, l'innovation et la nouveauté parviennent généralement à s'imposer ; mais il reste que la résistance qu'elles rencontrent rythme des dynamiques de développement naturellement mouvantes. L'économie, comme la société, 
ne se développe pas simplement à une allure régulière. Elle est, au contraire, constamment révolutionnée de l'intérieur par des initiatives nouvelles, c'est-àdire par lintrusion dans la structure productive, telle qu'elle existe à un moment donné, de nouvelles marchandises ou de nouvelles méthodes de production ou de nouvelles possibilités commerciales. Toutes les structures existantes et toutes les conditions de vie des affaires sont soumises à un processus de transformation continue. Toute situation est bouleversée avant qu'elle ait eu le temps de se réaliser complètement. Dans la société capitaliste, progrès économique est synonyme de bouleversement (Schumpeter 1947:53).

Les idées qu'avait proposées Schumpeter il y a plus d'un demi-siècle sont bien fondées et demeurent valables. Toutefois, la complexité des réalités économiques et sociales d'aujourd'hui rend nécessaire d'aller au-delà.

Malgré les critiques qu'on peut élever contre les idées de Schumpeter, sa contribution attire toujours l'attention, comme en fait foi la littérature scientifique. Le principe de "destruction créatrice" que Schumpeter avait avancé sert encore à articuler les idées des chercheurs et à mieux appréhender des réalités complexes. Ce principe désigne le processus par lequel les composantes internes d'une structure économique interagissent, permettant à celle-ci de se renouveler continuellement. Ce principe est utile pour qui veut comprendre comment l'évolution économique résulte souvent de mutations, de discontinuités et de ruptures. On doit à Schumpeter d'avoir formulé une vision novatrice des facteurs endogènes de développement d'une structure ou d'un modèle.

Néanmoins, bien que son auvre permette de mieux comprendre les mécanismes de l'évolution économique et sociale, les phénomènes étudiés par Schumpeter continuent, dans le présent contexte, de susciter un questionnement légitime.

\subsubsection{L'informatique à la source du changement technologique}

Depuis l'avènement de l'informatique, il n'est pas exagéré de parler de mutations dans l'activité économique et la vie sociale. Plusieurs chercheurs se sont efforcés, de divers points de vue, de saisir la nature et l'ampleur des changements observés. Malgré une réalité complexe difficilement appréhendable dans sa totalité, on peut tenter de dégager les grands axes de ces changements et d'en pressentir l'orientation. 
Le fonctionnement d'un système informatique met en œuvre deux groupes d'éléments complémentaires, l'un concret (le matériel), l'autre intangible (les logiciels). Mais si puissant soit-il, ce qui donne vie au matériel, c'est le logiciel. Entre eux s'exerce constamment une influence réciproque, le progrès de l'un se réalisant grâce au progrès de l'autre. Un logiciel est un ensemble d'informations, de règles codées dans un langage que l'ordinateur peut traduire en instructions lui permettant d'exécuter une tâche spécifique. Écrit dans un langage de programmation, "un logiciel est un texte actif» (Lévy 1992: 34), qui transforme les spécifications décrivant une fonction en une information sur la conduite d'un processus précis. Il «est à la fois une action et un processus [...] il est à la fois technologie et expression [...] il est un cas "d'espèce" qui comporte une signification prospective pour l'ensemble de l'industrie» (Zimmermann, $1995: 182$ ). On peut considérer le logiciel sous plusieurs aspects. Du point de vue industriel, il peut être utilisé pour régler le fonctionnement des moyens de production. Dans le commerce, il tient à jour l'inventaire de la marchandise, la liste des clients, la comptabilité. Il y a aussi le logiciel libre, dont le code source est en accès libre.

En somme, une infinité de logiciels affectés à des fonctions particulières: logiciels d'application, d'exploitation, de traitement de texte, d'enseignement, de jeu, antivirus, etc. On comprend pourquoi les logiciels sont une manifestation des avancées technologiques qui participent largement à forger l'ensemble des représentations sociales. Le logiciel est donc l'élément central du monde de l'informatique; il est indispensable au développement d'un matériel informatique de plus en plus sophistiqué et performant.

Aujourd'hui on ne peut guère trouver de secteur d'activité où l'informatique ne joue pas un rôle central, inévitablement accompagné d'un changement technologique. Mais il est difficile de se faire une idée claire de la façon dont un changement dans la technologie agit sur l'activité économique et la vie sociale. Plusieurs publications de l'OCDE (OCDE 1998; 2000, 2001 et 2004) sont consacrées aux changements technologiques, à leur influence sur les aptitudes et comportements des acteurs, et à leurs conséquences dans le temps.

Les travaux de Dosi montrent qu'un changement technologique a souvent des effets qui varient d'un contexte à l'autre. L'auteur avait déjà affirmé l'existence de liens forts entre le changement technologique et le développement économique et social (Dosi 1982). De plus, il 
a montré que le développement des systèmes techniques a des effets qui varient beaucoup, influençant inégalement l'organisation économique et sociale de l'activité humaine. Selon l'auteur, la diversité de ces systèmes et des choix stratégiques explique la variété des trajectoires technologiques. Une trajectoire technologique est «the pattern of 'normal' problem solving activity (i.e. of 'progress') on the ground of a technological paradigm [...] (où ce paradigme technologique se définit comme un) 'model' and a 'pattern' of solution of selected technological problems, based on selected principles derived from natural sciences and on selected material technologies» (Dosi 1982: 152). Un choix est jugé pertinent lorsqu'il reflète la capacité d'un acteur à identifier ses besoins de développement et à y apporter des solutions convenables. L'originalité d'une trajectoire technologique illustre la capacité des acteurs à promouvoir les dynamiques économiques et sociales d'un véritable développement.

De même, l'importance d'un changement technologique dépend du parcours socio-historique de la collectivité. Des atouts sociaux et historiques conditionnent les actions par lesquelles celle-ci s'approprie une technologie. Ils font qu'un paradigme technologique s'accompagne de certains mécanismes qui lui servent de supports et qui le présentent comme une réponse à des contraintes conjoncturelles ou structurelles. Les jeux de pouvoir liés à certains paradigmes technologiques orientent le changement vers certaines directions, et remettent en question d'autres directions qu'il faudrait alors négliger (Dosi 1982: 152). Souvent, ces jeux de pouvoir poussent une collectivité à choisir des stratégies susceptibles de l'aider à se doter de nouveaux atouts. Parfois, une nouvelle technologie atténue les inconvénients résultant du retard technologique, de l'éloignement, etc.

On le voit, Dosi appréhende les effets d'un changement technologique en l'inscrivant dans un cadre socio-historique plus large, et en le situant par rapport aux besoins exprimés par les individus et par les groupes qu'ils forment. Certes, un changement technologique a des effets qui ne résultent pas uniquement de la performance matérielle impliquée, mais surtout des actions et des choix humains.

L'apport de Dosi a constitué un point de départ pour plusieurs scientifiques, qui ont accordé un intérêt particulier aux changements technologiques. Par exemple, Nelson et Wright (1992) soutiennent que plusieurs indicateurs liés au parcours socio-historique de l'après-guerre 
expliquent la supériorité technologique des Etats-Unis, comparativement à d'autres pays comme le Japon, la France, l'Allemagne et la Russie. Généralement, ces indicateurs correspondent à l'utilisation des technologies dans divers secteurs d'activité économique, de même qu'à la capacité d'un pays ou d'un territoire à exporter ou à diffuser la technologie qui s'y est développée (Nelson et Wright 1992 : 1955). Les aptitudes d'un pays ou d'un territoire à importer les technologies qui ont fait leurs preuves ailleurs constituent un autre indicateur significatif, qui peut expliquer la supériorité d'un territoire ou d'une nation (Nelson et Wright 1992: 1956). Les exigences du nouvel ordre mondial qui s'est mis en place depuis le début des années 1990 renforcent l'importance de ces indicateurs, qui ont encouragé le déplacement rapide et plus fréquent des compétences et des connaissances (Nelson et Wright 1992: 1959).

II semble donc qu'un changement technologique peut avoir plusieurs sources et peut engendrer des conséquences variées et souvent imprévues. Lorsqu'une composante technologique paraît devoir être plus appropriée, elle scintille dans le ciel noir d'une conjoncture instable, génératrice de l'incertitude d'acteurs en quête de performance et de gain de productivité. Amin et Cohendet ont noté que les analyses économiques tendent à négliger la diversité des modes d'apprentissage et ils ont tenté d'en articuler les aspects économiques et sociologiques :

one of the strengths of our approach, we feel, has been to bridge the gap between economic and sociological/anthropological theorization of learning and coordination in organizations. Even the most evolutionary and situated economic accounts tend to stop short of explaining the everyday practices of learning in firms, and because of this, under-estimate the degree to which communities of practice bridge the divide between formal and informal learning, between situated and corporate-wide goals, and between exploration of competences and exploitation of routines (Amin et Cohendet 2000: 113).

Par ailleurs, les dynamiques économiques et sociales en place déterminent l'ampleur que prendra un changement technologique porteur d'une orientation originale. Ce changement s'opère ordinairement selon les mécanismes qu'adopte une collectivité, une organisation, une firme, pour résoudre les problèmes qu'occasionne son développement; c'est ainsi qu'elle apprend de ses propres expériences. 
Le développement de diverses formes de réseaux amène une collectivité à modeler son apprentissage sur l'effort collectif que fournissent ses principaux acteurs, désireux de s'adapter aux exigences conjoncturelles; ces réseaux et leur implication varient en fonction de l'histoire de cette collectivité (Wright $1997: 1564$ ). En fait, le parcours historique d'une collectivité et ses caractéristiques culturelles contribuent grandement à définir la manière dont elle s'approprie les technologies; cela explique souvent la coexistence dans une même collectivité de plusieurs niveaux et réseaux technologiques (Wright 1997 :1564).

Il est donc évident que les paradigmes et les trajectoires technologiques d'une collectivité changent et se développent. L'originalité des paradigmes et des trajectoires technologiques d'une collectivité a des effets importants sur ses activités économiques et sociales. Influencée par divers processus d'apprentissage, cette originalité peut multiplier les innovations et accroître les effets de la proximité. Selon Oinas, cette proximité, aux dimensions géographiques plus ou moins étendues, $s$ 'enracine surtout dans une terre sociale et culturelle. Si bien que la localisation de l'apprentissage et de l'innovation relie deux mondes: le géographique et le socioculturel, qui font que les interactions sont une source de prospérité (Oinas 1999 et 2000). Cependant, le développement des systèmes d'innovation et d'apprentissage n'est pas nécessairement limité par la localisation des activités économiques et sociales (Storper 1996: 787).

Ces considérations invitent à ne pas attendre d'un changement technologique qu'il ait toujours des effets identiques. La diffusion d'une technologie nouvelle rend celle-ci accessible à tout le monde, à condition d'avoir les moyens financiers, politiques et autres, de se l'approprier. Dans une note éditoriale, Greenaway (1994) avait constaté qu'à l'intérieur des pays membres de l'OCDE cette diffusion, tout comme l'innovation, est déterminée par l'importance des politiques concernant les technologies, l'éducation, etc. Il écrit :

Innovation and diffusion should be seen as being jointly determined by the evolution of markets and technology. Thus technology policy spills over into education policy, science policy and competition policy. As a result, policy makers must address the nature of the relationships between institutions, and the mechanisms for promoting user-supplier networks (Greenaway 1994: 917). 
Il semble done qu'un changement technologique n'arrive à changer l'environnement dans lequel il intervient que dans la mesure où les acteurs locaux et régionaux disposent des moyens et compétences nécessaires pour l'exploiter convenablement. Malgré les avantages qu'une collectivité peut tirer de l'appropriation de composantes technologiques, les effets de celles-ci sur la productivité, la croissance et le développement ne peuvent se sentir que dans les conditions où cette appropriation enrichit les normes, les valeurs et les activités traditionnelles. D'autant que les effets de la technologie condensent les interactions dans un territoire et poussent les acteurs à profiter des forces propres à leur histoire et à leur culture. L'appropriation d'une technologie suscite donc développement et gain de productivité, et on parle alors d'innovation. Mais implantée trop rapidement ou sans égard aux humains concernés, la plus belle technologie peut provoquer des ruptures et des crises.

La littérature spécialisée montre que dans tous les domaines d'activité économique : industrie, commerce, finance, gestion, communication, les changements technologiques offrent des possibilités inouïes à qui fait l'effort de les apprivoiser (OCDE 2004). Ils changent la paysage industriel, améliorent les pratiques commerciales, accélèrent l'échange d'information et incitent les travailleurs à se mieux qualifier pour répondre à un environnement exigeant. Lorsqu'une organisation adopte des technologies nouvelles, ses objectifs tendent à se modifier. Chaque changement semble agir positivement sur «the global objective function, the well-defined choice set, and the maximizing choice rationalization of firms actions» (Nelson et Winter 1982:14). En définitive, le changement est commandé par le besoin de l'organisation qui cherche à s'adapter à son environnement et aux exigences du marché. Constamment à la recherche d'un gain de productivité et de croissance, les décideurs les plus prometteurs sont ceux qui savent adopter la technologie appropriée au moment apportun.

Derrière tout changement technologique il y a une prise de décision qui implique non seulement les compétences des décideurs, mais surtout leur capacité à opter pour des stratégies adéquates. Quand il s'agit de l'appropriation technologique, la pertinence se mesure aussi par les effets de celle-ci sur les relations humaines. On estime généralement que les technologies ont des effets importants sur les rapports humains: politiques, sociaux et culturels, dans les pays industrialisés comme dans ceux en développement. On considère aussi que ces technologies bousculent les ordres sociaux établis, car elles remettent en question des organisations enracinées, comme l'Etat, l'entreprise, l'école, etc. Dans certains cas on peut 
obvier à ces difficultés par une implantation graduée ou sélective : "Technological frontiers are developed at different levels of technological sophistication, as older and newer technologies are often developed simultaneously to serve the needs of different customer groups» (Oinas et Malecki 2002: 107).

En définitive, les changements technologiques résultent, au départ, de décisions individuelles, reprises par consensus par un groupe ou une collectivité, afin d'améliorer ou changer un procédé, une situation qui n'est plus adaptée à la conjoncture ou aux objectifs poursuivis. L'amélioration des technologies et l'évolution technologique se conjuguent avec l'histoire humaine. De nos jours toutefois, l'avènement de l'informatique et l'usage diversifié des TIC accélèrent les exigences de changement et forcent les acteurs à une vigilance accrue, parfois à l'audace.

\subsubsection{L'influence d'Internet}

La littérature consultée montre que l'Internet constitue l'une des plus importantes avancées technologiques dont les dynamiques économique et sociale de développement aient subi l'influence. Plusieurs chercheurs se sont intéressés à ses effets. Les acteurs sont attirés par sa nouveauté, mais surtout par ses avantages : «agents are always capable of discovering new technologies, new behavioural patterns, new organizational set-ups. Hence, also the continuous apperance of various forms of novelty in the system» (Dosi 1997: 1531).

La généralisation d'Internet progresse à des rythmes variés. Relativement rapide dans certains milieux d'affaires et populaires, elle est plus lente dans d'autres. Il semble que persiste dans certains milieux d'affaires et populaires une méconnaissance, voire une certaine inquiétude vis-à-vis de l'ordinateur et d'Internet. Évidemment les coûts de l'équipement nécessaire, puis de l'abonnement à Internet, ne sont pas négligeables : «les pays où l'accès à Internet est relativement bon marché ont en général une plus forte densité d'hôtes Internet» (OCDE 1999 : 22). Internet a apporté avec lui certaines valeurs, «notamment les principes d'échange égalitaire et de circulation libre et gratuite de l'information, dans le cadre d'un réseau coopératif géré par ses utilisateurs» (Sicard et Mesnier 1998:138). Parlant de limportance d'Internet dans la vie économique, de la transformation qui s'opère dans le monde des affaires 
par le commerce électronique, Industrie Canada montre par des exemples concrets les avantages que les entreprises canadiennes peuvent y trouver:

amélioration du service à la clientèle, hausse de la part de marché, création de nouvelles possibilités de promotion et de distribution à l'extérieur des marchés locaux. Exécution plus rapide des commandes. Service après vente mieux adapté aux besoins. Possibilité rapide pour les clients de commenter les produits et services obtenus. Diminution des coûts et économie de temps grâce à l'amélioration des procédures internes. Diminution du nombre de réunions. Échange plus rapide de l'information. Élimination de la paperasse. Plus grande clarté des communications internes. Diminution des coûts et économie de temps grâce à une meilleure gestion intégrée production-distribution axée sur la demande-client. Intégration de l'organisation matérielle ou logistique et de la production finale aux activités préliminaires de mise en marché et de vente. Remise du contrôle de linventaire aux ordinateurs et aux programmes informatiques (http://com-e.ic.gc.ca/francais/stories/doc/e-busf.pdf) (Consulté en novembre 2001).

Ces propos illustrent la puissance de l'Internet comme une source de l'innovation économique, d'une économie nouvelle. Les écrits de Castells montrent que la nouvelle économie s'est bâtie autour de nouvelles règles et procédures de production, de gestion et de calcul économique. Selon l'auteur, cette économie n'aurait pu se constituer sans Internet. II ajoute que l'Internet permet à l'entreprise de disposer de «la capacité d'évoluer en liaison organique avec l'innovation, les systèmes de production et la demande du marché, tout en restant concentré sur l'objectif premier de toute entreprise: gagner de l'argent. Mais le problème, c'est que la façon dont on le gagne dans la Net-économie n'est pas aussi simple et directe qu'à l'ère industrielle - car les réseaux d'ordinateurs ont aussi transformé les marchés financiers, arbitres ultimes de la valeur de chaque firme» (Castells 2002: 99). De son côté, Porter montre comment les technologies de l'information modifient les forces concurrentielles :

elles dégèlent la structure de nombreuses industries, créant ainsi le besoin et la possibilité du changement: par exemple, elles accroissent le pouvoir des acheteurs dans les industries qui assemblent des composantes achetées à des tiers. La facturation des achats et les catalogues électroniques des foumisseurs aident les acheteurs à évaluer les sources de matières et à choisir d'acheter ou de fabriquer eux-mêmes; elles élèvent les barrières à l'entrée dans les cas où elles obligent à investir lourdement dans des logiciels complexes. Ainsi, les banques présentes sur le marché des services de gestion de trésorerie pour les entreprises 
ont désormais besoin de logiciels avancés pour foumir des relevés de compte par connexion; parfois, elles doivent aussi investir dans des ordinateurs et autres équipements de meilleur niveau; les systèmes de conception et de fabrication assistées par ordinateur influencent la menace de substitution dans bien des industries en permettant de renforcer les caractéristiques des produits plus vite, plus facilement et à moindre coût; l'automatisation du traitement des commandes et de la facturation a accru la concurrence dans de nombreuses industries de distribution. Les nouvelles technologies ont élevé les coûts fixes tout en se substituant aux hommes. Il en résulte que les distributeurs doivent souvent lutter plus dur pour accroitre leur volume (Porter 1999 : 95-96).

On discerne dans ces propos un fil conducteur : l'arrivée de l'Internet dans le monde des affaires change les dynamiques économiques, incite les acteurs à s'adapter à une réalité nouvelle, à une logique plus complexe. Tout comme les TIC, Internet change la situation non seulement par sa nouveauté, mais surtout en créant un contexte différent, mouvant, où les acteurs ont à constamment réviser leurs stratégies de développement.

Il semble donc que pour mesurer l'importance que revêt, pour une société, l'appropriation d'une technologie, l'Internet par exemple, deux indicateurs paraissent particulièrement importants. Un premier doit mesurer le taux de son utilisation. Un second doit évaluer les transformations que l'usage de cette technologie produit sur les attitudes et les comportements.

Le changement que montrent ces deux indicateurs facilite l'adaptation des individus et des acteurs de développement aux exigences d'une constante remise en question de l'ancien ordre de choses. 11 y a lieu de tenir compte de l'interactivité propre aux TIC dans l'évaluation du potentiel informationnel et communicationnel qu'introduit une technologie et particulièrement Internet. Dans ce cas, le branchement des ménages aux différents réseaux suscite un intérêt particulier, alors que s'établit une corrélation positive entre la demande de nouveaux services de communication, la libéralisation des marchés et l'introduction de nouvelles donnes sociales et culturelles. Les phénomènes qui résultent de ces transformations se généralisent avec le développement d'une infrastructure d'information, comme en témoignent divers rapports de l'OCDE. Dans l'un d'eux, on peut lire qu'«à mesure que la demande de nouveaux services de communication et de radioduffusion émanant des ménages et des entreprises s'élargit et que la libéralisation des marchés se poursuit, l'infrastructure de l'information, tant annoncée, commence à prendre forme» (OCDE 1999: 24). On peut lire ailleurs que «les nouvelles 
technologies pourraient sensiblement modifier les avantages dont jouissent les territoires, à moins que des mesures rapides ne soient prises en matière d'infrastructures de télécommunication, permettant à ces technologies de bénéficier à tous les habitants, qu'ils se trouvent en milieu urbain ou rural» (OCDE $2001: 27$ ).

Il parait évident que l'écart grandit entre groupes sociaux et entre nations. Castells explique cette inégalité par les pratiques des humains, qui sont dictées par un parcours socio-historique particulier, car «Internet est un réseau de communication planétaire, mais sa pratique, sa réalité en pleine évolution sont, je l'ai dit, les produits de l'action humaine dans des conditions historiques données» (Castells 2002: 16). De son côté, l'OCDE rapporte que ce développement inégal peut se manifester à l'intérieur d'un même pays :

«outre la fracture numérique territoriale, un autre fossé numérique pourrait se développer; cette-fois-ci entre les populations. L'accès aux services publics, à l'éducation, à la formation, à la documentation et en règle générale à la communication avec le monde pourraient bien être réservés, dans un premier temps, aux habitants des grandes villes, du moins à ceux qui ont les moyens de s'équiper en matériel informatique» (OCDE $2001: 27$ ).

Si le branchement aux réseaux d'information et de communication est un indicateur important, qui témoigne de l'insertion d'une communauté au sein de la société d'information, les modalités de cette insertion montrent la préparation individuelle et collective, souvent inégale, à en tirer profit.

Les pouvoirs publics ont vu la possibilité que donne Internet de stimuler la productivité dans tous les secteurs d'activité et d'accroître la compétitivité des entreprises (OCDE 2001 : 119). Ils ont encouragé le branchement à ce réseau et, effectivement, on assiste à une progression continue de l'appropriation des technologies de pointe. De nouvelles pratiques s'établissent, entraînées par l'appropriation des TIC et le branchement à Internet, désormais essentiels à l'innovation et à la prospérité des affaires (OCDE 2000 et 2001). Les acteurs de développement sont encouragés par les avantages de ces technologies: «le courrier électronique et l'Internet peuvent améliorer les communications avec l'extérieur - autres entreprises ou consommateurs - réduire les coûts des transactions, accrôtre leur vitesse et leur fiabilité et extraire un maximum de valeur de chaque transaction de la chaîne de valeur » (OCDE $2004: 12)$. 
Toutefois on peut avancer que toutes les sociétés n'ont pas la même aisance à intégrer les nouvelles technologies et à les utiliser pour leur développement. Pourtant, ces différences influent inévitablement sur le fonctionnement des économies, de même que sur les dynamiques sociales et culturelles, et tendent à s'accentuer à mesure que ces technologies se perfectionnent. Les études portant sur les changements technologiques montrent qu'une réelle innovation, celle qui transforme des façons ancrées de penser et de faire, suscite d'abord la curiosité des acteurs, puis le désir de l'adopter pour l'avancement de leurs affaires. Des périodes d'ajustement social sont par la suite nécessaires pour qu'apparaissent dans la vie quotidienne les avantages apportés par le changement.

Pour qui veut saisir l'influence des technologies sur le développement des économies et des sociétés, il est utile de s'attarder à l'analyse du pouvoir de ces technologies d'agir sur les dynamiques en place d'en orienter l'évolution. A cette fin, il y a lieu de voir comment cette influence se traduit dans les pratiques entrepreneuriales.

\subsection{Le changement technologique et l'entreprise}

La portée d'un changement technologique n'est pas nécessairement évidente et des questions demeurent pendantes. Apparentées, mais difficilement mesurables, les conséquences et l'influence d'un changement technologique diffèrent selon les dynamiques économiques et sociales existantes. Cette réalité conduit, dans un premier temps, à s'intéresser aux effets de ces changements sur le monde des affaires.

\subsubsection{Effets des changements technologiques dans le monde des affaires}

Une lecture attentive de la littérature spécialisée permet d'avancer que, dans le monde des affaires, le changement technologique crée une situation nouvelle qui incite les entreprises à s'ajuster à la dynamique d'un marché de plus en plus international (OCDE 2004). L'entreprise n'existe pas dans un vacuum. Bien que les décisions concernant l'adoption d'une technologie soient en principe libres et puissent varier selon les acteurs et leurs compétences, il importe de prendre en considération le contexte, particulièrement le marché, qui a sa propre logique, impose certaines orientations et en écarte d'autres: «Even when the initial path of technological development is generated by "technology push", factor market forces often act 
to modify the path of technical change» (Ruttan 1997: 1524). Les lois du marché et ses fluctuations influent nécessairement sur les acteurs et les incitent à bien peser leurs décisions quant au choix d'une nouvelle technologie.

Dans un monde oủ la compétition est rude et s'internationalise, l'acteur qui veut maintenir un avantage concurrentiel a tout intérêt à faire des choix technologiques bien étudiés afin, entre autres, de pouvoir y adapter sans trop de problèmes le savoir-faire existant aux nouvelles conditions: «We live in a turbulent world dominated by an increasing rate of technological change. Economic agents, including firms and governments, are forced to adapt to technological change in order to survive in a competitive environment» (Archibugi, Howells et Michie 1999 :13). La décision d'un acteur d'implanter ou non une technologie de pointe, et le choix de la façon dont le matériel technologique sera utilisé peuvent décider de l'avenir, sinon de la survie de l'entreprise.

En effet, l'abondance et la spécialisation des logiciels, l'évolution en puissance et en polyvalence des matériels, l'application croissante de la numérisation à toutes sortes de tâches, enfin la concurrence devenue planétaire, toutes ces influences convergent vers l'accélération de l'innovation; tout en améliorant «les méthodes de fabrication des produits existants, (elles) encouragent la fabrication de produits possédant de nouvelles caractéristiques importantes et développent de nouvelles technologies d'organisation, de commercialisation et de gestion» (Ratti 1992: 4). N'est-ce pas là l'image d'une mer agitée, semée de récifs, mais bordée de riants rivages?

L'importance des changements technologiques a poussé nombre de scientifiques à s'intéresser à leurs effets sur la productivité des employés et des entreprises. Par exemple, Bill et Lichtenberg (1999) se sont intéressés aux TIC et à leurs conséquences sur la productivité. À cette fin, ils ont analysé une information variée, publique ou confidentielle, sur la diffusion et l'utilisation des ordinateurs dans les grandes entreprises. Ils ont exploité cette information pour voir comment l'usage des TIC influence la productivité des employés. Les résultats font voir que la productivité est fortement liée au nombre d'ordinateurs individuels utilisés dans une entreprise, et que la puissance des composantes technologiques importe moins que la façon de s'en servir. Les auteurs ont aussi démontré qu'il n'y a pas de différence, quant à la valeur du travail, entre l'usage et l'absence des ordinateurs. 
De leur côté, Licht et Moch (1999) se sont intéressés à l'influence de l'innovation technologique sur le secteur des services, et plus particulièrement sur les liens qui existent entre la qualité des services offerts par une entreprise et sa productivité. II ont montré qu'il existe des corrélations positives entre la qualité des services, le capital investi par l'entreprise dans les activités de R\&D et dans la formation de son capital humain. Selon les auteurs, bien qu'une innovation technologique améliore la qualité des services offerts par une entreprise, elle n'influence pas nécessairement la productivité de l'entreprise (Licht et Moch 1999: 377 ). Dans le même ordre d'idées, Blundell et al. (1999) ont étudié l'innovation technologique dans des entreprises intemationales qui offrent des services en ligne. Ils ont démontré qu'une innovation développée par une entreprise a souvent des conséquences qui dépendent largement du marché de celle-ci. Plus récemment, Porter (2003) présente une vision originale des effets des TIC sur les dynamiques économiques d'un territoire. Il avance trois hypothèses, à la lumière desquelles on peut appréhender le développement économique : 1 . Innovation is vital for long-term increases in productivity; 2 . Innovation is more than just scientific discovery and 3. There are no low-tech industries, only low-tech firms (Porter 2003 b). La combinaison de ces trois hypothèses permet à l'auteur d'établir une relation de cause à effet, d'une part entre l'innovation technologique et la productivité et, d'autre part, entre la productivité et la prospérité.

La plupart des écrits que nous venons d'aborder montrent une réalité importante : quand on se l'est appropriée convenablement, une technologie diminue les coûts d'opération par l'efficience et la rapidité accrues des moyens de production, et la qualité constante du produit. Ces résultats favorables sont le fruit de décisions judicieuses prises en amont. L'implantation d'une nouvelle technologie résulte en effet d'un processus vital pour l'entreprise : la prise de décision. Diverses raisons sous-tendent la décision d'un dirigeant d'investir pour un changement technologique d'importance. L'objectif peut être d'améliorer sa gestion interne, affermir sa capacité concurrentielle, corriger un problème environnemental, etc. La théorie économique classique rend compte du processus menant à ce genre de décision. Elle postule que le décideur est essentiellement rationnel, impartial, uniquement préoccupé d'efficacité et d'efficience ; qu'il décide après avoir supputé tous les aspects d'un projet, en fonction de son utilité. Sa décision est donc, en principe, parfaitement rationnelle, pragmatique. 
Les prémisses qui fondent cette théorie ont été remises en question, par exemple, par Daniel Kahneman, économiste et psychologue, prix Nobel d'économie en 2002. Ses recherches résumées dans sa "théorie des perspectives" (Prospect theory) montrent que les décisions de nature économique reposent bien souvent sur des critères peu sûrs, voire erronés, où l'émotivité peut avoir une part importante. En effet, dans les situations complexes, mouvantes, où l'incertitude est grande, la rationalité pure, la rectitude du jugement sont souvent altérées, à l'insu du décideur.

Le risque d'interférence d'origine émotionnelle est plus grand lorsqu'il s'agit d'une décision individuelle. Dans le cas d'une entreprise, la possibilité d'être influencé par l'émotion semble réduite, parce qu'ordinairement une question importante est passée au tamis de plusieurs personnes avant la prise de décision finale. Ces considérations sont, pour le dirigeant, un appel à la vigilance.

\subsubsection{Dimensions économiques d'un changement technologique}

Depuis les années 1970, les changements technologiques sont devenus un phénomène important et quasi continuel qui a attiré l'attention des chercheurs. Leurs études montrent que ces changements apportent des améliorations significatives en matière de croissance, d'emploi et de productivité. Les réflexions de Freeman (1987) font partie de ces travaux. L'auteur a montré comment un changement technologique se déroule en plusieurs étapes, allant de l'invention et de la créativité jusqu'à la diffusion des innovations conséquentes. Le passage d'une étape à une autre dépend de certains facteurs, notamment ceux qui favorisent la diffusion de la science et de la technologie, qui agissent non seulement sur le fonctionnement de l'entreprise, mais aussi sur ses relations avec son environnement.

La succession des changements technologiques a principalement modifié les relations qu'entretiennent les entreprises avec les éléments de leur environnement. Ratti constate qu'autour de toute entreprise existent des "espaces fonctionnels" substantiellement modifiés par les changements technologiques : l'espace de production, l'espace de marché et l'espace de soutien. La signification des deux premiers est immédiatement évidente. Quant à l'espace de soutien, on le définit "par un ensemble de relations "hors marché", ou précédant le marché, et qui expriment l'orientation stratégique de l'entreprise vis-à-vis de son 
environnement extérieur» (Ratti 1992: 55). Pour obvier aux contraintes éprouvées par son entreprise, le dirigeant en explore les aspects utiles à sa stratégie. 11 est ainsi amené, par le renouveau technologique, à s'harmoniser avec la tendance qui conduit à la prééminence de valeurs et normes planétaires, tout en ayant la sagesse de ne pas négliger le soutien rapproché de son milieu et de la dynamique locale de développement.

L'étude du changement technologique ne réfère plus seulement à l'évolution des outils de travail et des moyens de production, mais renvoie surtout à la diffusion des connaissances, du savoir, de la science et des compétences (OCDE 2001 :119). Plusieurs scientifiques ont déjà foumi des efforts importants dans ce sens. Ils ont montré comment se produit l'adaptation au changement technologique. Souvent, l'adaptation est amorcée dans la perspective de mettre les connaissances, le savoir et la science au service des activités nouvelles. Les acteurs y trouvent des occasions d'apprentissage, menant à de nouvelles compétences, utiles à l'exploitation de la technologie concernée (Jones et Newman 1995 : 898). Dans leur étude de cas, les auteurs ont démontré qu'un changement technologique a lieu selon certains processus : rejet des pratiques accoutumées, devenues caduques, et adoption de pratiques nouvelles. Ces deux processus, que les auteurs ont essayé d'appréhender à partir du principe de "destruction constructive" de Schumpeter, sont séparés par une période courte d'adaptation.

A la base de leur décision d'implanter une nouvelle technologie, les acteurs sont raisonnablement sûrs de profiter de certains avantages: hausse de la productivité, de la capacité concurrentielle, etc. et, à terme, ils escomptent un retour sur leur investissement. Cette décision ne va pas sans hésitation et il arrive qu'une firme refuse de suivre le changement à répétition et préfère attendre, considérant que les avantages prévisibles ne permettront pas de récupérer le capital engagé. Les auteurs écrivent :

We show the conditions under which 'incessant' technology change can arise, but we also show that inertial responses are an equally interesting implication when considerations of foresight and rational learning are made explicit. This arises because the incentives to absorb new technologies in general conflict with the incentives to match well with them when technologies thus can be explained either as a rational way of capturing the benefits of better matching in the future, or as a consequence of lock-in effects, which make it too expensive to release adaptive capital already accumulated (Jones et Newman 1995: 912). 
On voit 1 importance, pour un décideur, de bien évaluer les conditions d'implantation et les suites d'un changement technologique. Mais une fois résolues les modalités d'acquisition et d'appropriation, les acteurs ont à leur disposition une technologie propice à la croissance et à la productivité, surtout si le contexte, géographique, historique, social s'y prête. Toutefois quel que soit le contexte, l'adoption d'une technologie complexe gagne à se réaliser selon une démarche simple et graduée, se gardant d'écueils toujours possibles. Singh (1997) a mené une recherche à cet égard, qui lui a permis de confirmer les hypothèses suivantes: 1 . The greater the complexity of technology that a business develops, the greater its risk of failure. 2 . Technological change is positively associated with the risk of business failure. 3 . Technological expansion is positively associated with the risk of business failure. 4 . The greater the diversity of technology that a business develops, the greater its risk of failure (Singh 1997).

De son côté, Ruttan (2002) s'est intéressé aux rapports existant entre les technologies, la croissance et le développement. Il a analysé les effets des technologies dans cinq secteurs importants: l'agriculture, l'électricité, l'industrie chimique, l'informatique et la biotechnologie, et a montré que l'ampleur et la direction d'un changement technologique résultent d'ordinaire d'une mise en valeur des ressources (humaines, techniques et autres) disponibles, et de l'innovation institutionnelle. Selon l'auteur, la compétitivité, l'environnement, les politiques concernant la science et la technologie, et l'aptitude à intégrer l'ordre global qui se met en place, sont autant de facteurs qui décident de l'ampleur et de la direction qu'impose un changement technologique. La combinaison de ces facteurs peut être à l'origine d'une remise en question de l'ordre institutionnel existant et encourager l'émergence d'un autre régime plus adapté aux exigences conjoncturelles. L'auteur a repris cette idée dans un travail subséquent, en 2003, où il a montré que les jeux de pouvoir, occasionnés par l'innovation institutionnelle, conditionnent le changement technologique. Lorsque les ressources locales et régionales sont valorisées convenablement, en même temps que les aspects positifs de la culture, ces jeux de pouvoir accélèrent et orientent le changement technologique. Selon l'auteur, lorsque la combinaison de ces facteurs créée un déséquilibre important et ébranle l'ordre existant, alors s'ouvrent des nouvelles perspectives et divers aménagements deviennent possibles. 
Bref, une technologie n'arrive pas seule dans un milieu; elle porte avec elle une certaine culture, des attitudes, des comportements qu'il faut acquérir patiemment. Les dynamiques conséquentes à un changement technologique sont donc responsables de la direction que prend le développement des activités humaines. Il y a là, bien sûr, une saine mise en garde, un caveat emptor que tout entrepreneur doit considérer, sans toutefois se laisser décourager d'investir dans une nouvelle technologie. Car la récompense d'un changement technologique réussi peut être grande.

\subsubsection{Effets des changements technologiques sur la socialisation}

L'introduction d'une technologie nouvelle et sa propagation dans un territoire ont des répercussions sociales profondes, où l'on peut distinguer de nouvelles formes de socialisation. Une littérature importante montre que l'appropriation des TIC a de tels effets. Werner Rammert (1997) s'est intéressé à l'analyse des changements technologiques et à leurs effets sur les pratiques sociales. Il a mis en relief des corrélations importantes entre un changement technologique et le développement des interactions entre acteurs. Il montre que ces derniers, lorsqu'ils sont établis dans un cadre local bien circonscrit, s'approprient une technologie donnée et adaptent, ce faisant, les structures sociales locales à des exigences de niveau global. Toutefois même avec une planification préétablie, l'issue n'est guère prévisible. Selon l'auteur, «each action and each connection may be planned with deliberation and strategy; but the variation of a technology project is a blind one in the long run and on the global level» (Rammert $1997: 184$ ).

Le développement technologique à long terme ne peut pas s'expliquer uniquement par l'accumulation des stratégies individuelles, car le fonctionnement des cadres institutionnels et sociaux en place contribue au devenir technologique d'une société (Rammert 1997 : 185). C'est donc la capacité des institutions d'un territoire à s'ouvrir à une nouvelle technologie, qui permet à cette technologie éventuellement de pénétrer et d'influencer la vie sociale et culturelle.

Pour Bac (2000) et Acemoglu (2001), les nouvelles formes de socialisation se développent d'abord dans le monde du travail. La coexistence sociale des «Good Jobs» versus les «Bad Jobs» montre que plus les individus s'approprient les TIC, plus ils ont la chance d'être mieux 
rémunérés et donc de mener une vie de meilleure qualité (Acemoglu 2001). La coexistence sociale d'emplois «de qualité» ou «médiocres» montre, comme corollaire, la précarité d'emplois qui exigent beaucoup d'efforts et de performance de la part des employés; ce qui tend à créer des déséquilibres dans le marché du travail (Bac $2000: 696$ ). Ces contraintes, nouvelles et anciennes, qui marquent le fonctionnement du monde du travail, affectent d'une manière ou d'une autre la vie sociale.

De leur côté, Bartel et Sicheman (1993 et 1999) ont montré comment l'usage d'une technologie influe sur les salaires, et par conséquent le mode de vie des travailleurs. Krueger (1993) a établi que les travailleurs qui utilisent des ordinateurs gagnent $15 \%$ de plus que ceux qui n'en utilisent pas, que les étudiants diplômés d'une institution de l'enseignement supérieur utilisent davantage les ordinateurs. Cela se traduit par une demande sociale accrue pour les métiers, et en conséquence les pratiques, qui exigent le recours aux TIC.

Des conclusions semblables ressortent d'un travail qu'Allen a consacré aux effets des technologies sur les structures salariales de différents secteurs d'activité économique. Une observation importante de l'auteur est le lien qu'il a démontré entre l'usage des objets technologiques et les différentes catégories d'employés, oeuvrant dans divers secteurs manufacturiers et autres. Il apparaît ainsi que les pratiques des employés de tous les âges changent profondément par l'introduction de la technologie nouvelle (Allen $2001: 465$ ).

On peut inférer de ces recherches qu'il y a une socialisation qui s'opère à mesure que le savoir-faire, porté par les objets issus de la nouvelle technologie, agit sur les pratiques sociales ; toutefois il est bien difficile, sinon impossible de saisir exactement comment ce savoir-faire se développe. Il introduit en effet une seconde forme de socialisation, qui se manifeste à la suite de réglages inconscients, donc sans s'appuyer sur des règles prédéterminées. Ainsi, il est remarquable de voir comment l'acculturation liée au développement du savoir-faire se produit facilement et rapidement dans la vie quotidienne. On utilise un grand nombre d'objets sans penser à la somme des connaissances nécessaires à leur production. C'est particulièrement le cas des TIC. Les nouvelles façons de communiquer qu'elles permettent transforment 
radicalement l'espace et le temps, dimensions fondamentales de l'expérience humaine. Les lieux perdent la substance même de leur signification culturelle, historique et géographique, pour être intégrés dans des réseaux fonctionnels produisant un espace de flux qui se substitue à l'espace des lieux. Le temps luimême est effacé lorsque le passé, le présent et l'avenir peuvent être programmés à interagir les uns avec les autres en un même message. L'espace des flux et le temps intemporel sont ainsi les fondements matériels d'une nouvelle culture, laquelle transcende et intègre la diversité des systèmes de représentation transmis par lhistoire: la culture de la virtualité réelle où le simulacre est la réalité en gestation (Castells $1998: 424$ ).

Cette remarque peut s'appliquer particulièrement bien à la jeune génération, celle qui est née à l'ère des TIC. Les adultes d'un certain âge ne cessent de s'étonner de la facilité et de la rapidité avec lesquelles cette génération arrive à s'approprier n'importe quelle composante informatique et à s'en servir de façon inventive. Pour eux, cela va de soi. Il y a pourtant là un phénomène d'acculturation très important, aux répercussions sociales considérables, qu'on ne fait que commencer à mesurer.

Que ce soit insensiblement ou par à-coups, chacune des composantes issues de la numérisation a des conséquences pour la société; chacune contribue à modifier le fonctionnement économique et social. Il apparaît ainsi que chaque composante informatique opère dans un territoire, et sur chaque acteur, un ensemble de changements; et tout changement, si minime soit-il, s'effectue naturellement au rythme même de la vie quotidienne où s'intègre cette composante. On en arrive donc à voir dans la définition d'une composante numérique la révélation à la fois de son utilité et de sa capacité d'insertion dans l'activité économique et sociale. Une innovation peut s'imposer très rapidement, par sa seule nouveauté, à une fraction de la population, mais sa vie risque d'être éphémère. Mais si elle est utile et embellit l'existence, elle se généralisera et durera.

Plus ou moins consciemment, les acteurs importants dans un milieu, puis la population, adoptent les standards que créent naturellement les applications technologiques de la science. Ces standards représentent ce qu'est penser et agir conformément aux exigences de la conjoncture. On peut voir là l'une des raisons du succès souvent rapide d'une innovation et qui font des technologies le principal outil qui oriente le développement. Les retombées sociales conséquentes à la numérisation et à ses applications sont particulièrement visibles et montrent qu'on aime bien utiliser les nouveaux objets pour leur utilité. 
De ce qui précède, trois idées principales paraissent se dégager. D'abord, l'usage des objets fondés sur une haute technologie, particulièrement de l'informatique, encourage l'adoption de stratégies individuelles et collectives de développement, de façon à suivre les vagues de l'innovation. Ensuite, cet usage revêt un aspect social, porteur de normes et de valeurs propices à l'activité scientifique. Enfin, cet usage commande une expertise professionnelle originale, sans nécessairement devoir renoncer à l'attribution de nouvelles vocations à des techniques éprouvées.

Parmi les retombées de la numérisation, les technologies de l'information et de la communication sont à l'avant-plan. L'usage des TIC demeure cependant encore inégal parce que son appropriation, à partir d'une technologie se renouvelant sans cesse, exige l'acquisition de connaissances et d'un savoir-faire nouveau, et impose en même temps des ajustements dans l'entreprise et dans la société. Par suite des progrès technologiques, de la multiplication de produits et services inédits, la société pénètre dans un monde nouveau, complexe et séducteur, qui la transforme. Ce changement exprime le passage des sociétés, d'une époque où l'influence du potentiel technologique ne dépassait guère les frontières nationales d'un pays, à une autre période où la globalisation a rendu les systèmes technologiques ouverts sur des ensembles plus vastes. Wright rend compte de cette réalité et écrit:

In an era of pre-scientific technology, technical knowledge tended to be much more tacit, informal, and location-specific, and therfore national in character. Richard Nelson and I argue that this national technologies distinctiveness has eroded in the modern era of science-based technologies, and that much of the apparent loss of American technological leadership reflects the deeper globalization of technology itself (Wright 1997: 1565).

Globalisation de l'économie, mondialisation des échanges, ces suites de la révolution numérique et des changements technologiques, manifestes au niveau macroéconomique, ont néanmoins leurs répercussions au niveau local et, finalement, au plan individuel. C'est pourquoi la compréhension des changements technologiques et de leurs conséquences ne saurait commencer sans d'abord s'intéresser à l'usage que fait l'Homme des objets nouveaux à sa disposition. La science, et la technologie qu'elle engendre, permettent «à l'Homme, en lui fournissant des outils toujours plus performants, de construire un univers socio-économique 
propre, et d'innover par rapport à cette constructiony (Caccomo 1996: 17). L'Homme doit demeurer la raison d'être, le point de départ et d'arrivée de la technique.

Les deux premières sections de ce chapitre ont ceci d'intéressant qu'elles mettent en évidence au moins deux conclusions importantes. D'abord tout changement technologique ne peut avoir des conséquences heureuses que s'il s'inscrit dans une stratégie globale de développement. Or pour qu'une telle stratégie prenne forme, il faut que les acteurs de développement, qu'ils soient individuels ou collectifs, prennent conscience du pouvoir des technologies de pointe de stimuler les spécificités d'un territoire. À cet égard, la littérature témoigne que le développement récent de l'informatique et l'usage progressif de l'Internet ne sont que des facettes, pas nécessairement les plus spectaculaires, des profondes transformations économiques et sociales en cours dans plusieurs régions de la planète. En second lieu, ces transformations apparaissent davantage et en premier dans le monde des affaires, car les entrepreneurs cherchent constamment à suivre les vagues du renouvellement technologique, pour acquérir ou préserver un précieux avantage concurrentiel. C'est aussi dire que les changements technologiques ont d'abord une dimension économique considérable, qui n'occulte pas cependant leur forte incidence sur le développement des formes nouvelles de socialisation.

II ressort de la littérature que pour mieux connaître la contribution des TIC et des changements technologiques à l'activation des avantages d'un territoire, il faut s'efforcer de comprendre comment les structures en place conditionnent l'appropriation des TIC et, surtout, comment une conjoncture donnée façonne cette appropriation.

\subsection{Exigences conjoncturelles et TIC}

L'analyse des exigences conjoncturelles conduit à traiter de questions principalement liées au développement de certains phénomènes relatifs aux TIC et à l'accélération du temps. La réponse à ces questions amène à tenter de mesurer l'emprise du savoir scientifique et de la nouvelle économie. 


\subsubsection{L'influence des TIC}

En croissance continue, le savoir scientifique et les changements technologiques qui l'accompagnent, poussent les individus et les groupes à changer leurs attitudes et leur mode de vie. Mais tout changement comporte une part d'inconnu. D'un côté il importe, pour raison de compétitivité, de s'adapter aux vagues successives de la nouveauté technologique; de l'autre, on cherche à préserver les caractéristiques qui marquent l'identité. A la recherche de cet équilibre, le développement harmonieux de l'économie et de la société ne saurait guère se réaliser sans d'abord la maîtrise des dynamiques propres au groupe et au territoire, sans aussi une familiarité suffisante avec le monde technologique et en particulier les TIC.

L'application des technologies de l'information, devenue si courante à notre époque, suggère à Porter que ce qui fait la différence entre les régions et entre les nations, c'est l'ingéniosité à mettre ces technologies au service des secteurs économiques existants. Il estime que tous ces secteurs sont aptes à intégrer les technologies de pointe, et à les exploiter pour augmenter la productivité : «la productivité est ce qui détermine la prospérité» (Porter 2003a). L'auteur met l'accent sur la relation entre la compétitivité, la productivité et la prospérité : «le point de départ pour penser à la compétitivité est que la prospérité de n'importe quel secteur est fondamentalement conduite par la productivité» (Porter 2003a).

Cependant, il faut souligner qu'il est souvent difficile de mesurer la productivité, surtout dans le secteur des services, où l'on utilise davantage les TIC (Wolff 1999: 303). L'étude de Triplett (1999) confirme l'existence de certains avantages associés à l'usage des nouvelles technologies. Sa recherche a tenté d'illustrer la capacité des ordinateurs à influencer la croissance économique. Selon l'auteur, bien qu'il existe des liens entre l'appropriation des technologies et l'augmentation de productivité, ces technologies ne permettent pas d'atteindre complètement les objectifs visés par les acteurs : «computers are less productive than they were thought to be when decisions were made to computerize» (Triplett 1999:326). Au contraire, Lehr et Lichtenberg confirment l'existence de liens forts entre l'appropriation des ordinateurs et la croissance économique; ils soulignent cependant que pour arriver à une évaluation correcte de ces liens, 11 importe de disposer des outils pertinents. Ils écrivent: «Our principal finding, that computers - especially personal computers - do contribute positively to 
productivity growth, suggests that the traditional Information productivity paradox is largely a measurement problem» (Lehr et Lichtenberg $1999: 335$ ).

Bien qu'il ne soit pas toujours facile d'en mesurer les avantages, l'informatisation est désormais indispensable dans l'entreprise. Bouchy constate que, «comme toute révolution industrielle le permet, l'informatique innerve tous les aspects de la vie quotidienne et sans rupture, de la vie familiale à la vie professionnelle, la collectivité est entrée dans un monde où l'informatique devient l'outil privilégié de l'activité des entreprises et de la communication des hommes» (Bouchy $1994: 25$ ).

La croissance de la productivité dépend de plusieurs facteurs, mais il semble bien que les ordinateurs et les logiciels qui les gouvernent - et ce qu'on en fait, peuvent faire la différence entre la croissance et la stagnation ou le déclin. L'informatisation a rendu possible l'automatisation, qui se charge dorénavant d'une foule de tâches, itératives, dangereuses ou peu valorisantes, permettant «de concentrer les efforts de production des professionnels sur les tâches à forte valeur ajoutée, d'assurer la qualité des procédés et des produits et de produire dans des conditions de coûts et de délais connues à l'avance» (Bouchy 1994 : 24).

Il est un peu étonnant qu'on ait attendu que l'automatisation se généralise dans le domaine de la production, pour commencer à s'intéresser aux possibilités offertes par les TIC dans le monde des affaires. On a compris qu'il ne suffit pas de s'approprier quelques composantes technologiques destinées à la production, pour pouvoir profiter pleinement du potentiel de l'automatisation et rehausser la productivité. Il fallait joindre à ces composantes les technologies de l'information et de la communication, et la force de l'Internet, pour faire une entrée réussie dans la nouvelle économie et s'assurer d'y subsister. Pour la nouvelle économie, en effet, le principal outil est le numérique et ses applications, dont au premier chef les TIC. Castells s'anime en parlant de cette économie, aussi exigeante que gratifiante : elle est «fondée sur un potentiel sans précédent de croissance de la productivité, résultant de l'usage d'Internet pour toutes sortes d'entreprises engagées dans toutes sortes d'opérations; il est probable que nous entrons dans un monde économique nouveau. Ce monde n'abolit pas le cycle des affaires, ne réduit pas à néant les lois économiques, mais transforme leurs modalités et leurs effets, tout en enrichissant les règles du jeu» (Castells $2002: 14$ ). 
L'essor récent des TIC change et complexifie les structures sociales et économiques accoutumées. L'interpénétration croissante de ces structures brouille les frontières qui séparent des champs sociaux et économiques coexistants. C'est pourquoi il est difficile de saisir comment les TIC participent aux dynamiques économiques et sociales en place. En fait, si les formes de la coexistence, entre champs sociaux et économiques, apparaissent peu précises, c'est que notre société est «à la fois capitaliste et informationnelle, quand bien même elle présente des variations historiques considérables d'un pays à l'autre, selon l'histoire de chacun, sa culture, ses institutions et les relations spécifiques qu'il entretient avec le capitalisme global et la technologie de l'information» (Castells 1998 : 34).

L'examen de la littérature spécialisée permet de rapporter certaines initiatives intéressantes, visant à cerner les effets des TIC sur les dynamiques économiques et sociales de développement. Une contribution de Foray et Steinmueller (2003) décompose en deux phases le processus par lequel les TIC influent sur les dynamiques de développement. Pour reprendre le vocabulaire des auteurs, c'est à travers ces deux phases que s'opère l'inscription dans la scène économique et sociale du savoir. Dans un premier temps, l'usage des TIC tend à étoffer les connaissances et le savoir des utilisateurs, qui sont dès lors amenés à activer un ensemble de routines propres aux organisations en place, ce qui contribue à enrichir la vie quotidienne et à en dégager les tendances dominantes. Dans un deuxième temps, l'ensemble des représentations qui émergent ainsi ouvre devant les individus des possibilités qu'il leur appartient d'exploiter et d'orienter selon leurs intérêts. C'est ainsi qu'un nouvel ordre technologique prend forme, que des voies de développement s'ouvrent devant les collectivités, que s'imbrique dans les structures et la conjoncture du moment, la nouvelle donne technologique.

Les acteurs ne peuvent plus échapper aux impératifs technologiques et informationnels et ils ne peuvent plus les ignorer lorsqu'ils fixent leurs choix stratégiques. L'inquiétude, qui peut aller jusqu'à l'anxiété, guette les acteurs de développement, car ils craignent ne pas pouvoir suivre les vagues du changement: «la peur du changement est une constante historique de l'expérience humaine (tout comme, paradoxalement, la pression des audacieux pour que ça change), mais j'estime que si cette résistance, cette insatisfaction face au monde des réseaux dynamisé par Internet existe, c'est que plusieurs défis n'ont pas été relevés) (Castells 2001 : 335). Ces défis demeurent quand on n'arrive pas à reconnaitre et à utiliser le pouvoir foncier 
des TIC d'améliorer les conditions de vie des individus, des entreprises et des territoires, en créant de nouvelles dynamiques, des interactions originales génératrices d'innovation.

L'introduction d'une technologie inédite crée une certaine effervescence dont peut souffrir temporairement la dynamique économique. En même temps que l'attente d'avantages probables, il y a l'appréhension vague d'une désillusion ou d'un bouleversement irréversible des structures accoutumées. Toutefois pour l'acteur prudent, qui n'a pas craint la nouveauté et a su miser sur la communication et le savoir, les avantages peuvent être considérables : «dans le nouveau mode informationnel de développement, c'est la technologie de la production du savoir, du traitement de l'information et de la communication des symboles qui engendre la productivité» (Castells $1998: 38$ ). On peut donc s'attendre à ce que l'usage approprié de ces technologies devienne source de richesse accrue dans un territoire, parce qu'elles encouragent l'innovation, augmentent la compétitivité des entreprises et soutiennent leur avantage concurrentiel. En fait, les TIC semblent prendre dans la société une place de plus en plus importante :

Not only can the application of IT provide better ratios of value rated to effort expended in established processes for producing goods and delivering services, but it can also reframe and redirect the expenditure of humain effort, generating unanticipated payoffs of exceptionally high value. Information technology can support inventive and creative practice in the arts, design, science, engineering, education, and business (Mitchell et al. 2003: 15).

Les TIC semblent créer des dynamiques nouvelles: elles consolident les activités entrepreneuriales car elles incitent les entrepreneurs à s'encourager et à se motiver (Mitchell et al. 2003: 19); elles favorisent la généralisation de la créativité en stimulant l'interdépendance des activités culturelles, des dynamiques du marché et des politiques mises en place afin d'orienter le développement d'une nation (Mitchell et al. 2003: 21-22).

Il semble donc que les territoires périphériques, s'ils savent tirer parti de la nouvelle dynamique, peuvent faire leur place dans un contexte de mondialisation.

En mettant à la disposition des acteurs le savoir du monde, les TIC confortent leurs aspirations et leur offrent la possibilité d'élargir les frontières de l'agir. La mutation récente de l'économie est largement tributaire de ces nouveaux instruments. Castells remarque que 
cette économie, «dont le fer de lance est le cybermonde des affaires, n'est pas une économie en ligne, mais une économie propulsée par les technologies de l'information, reposant sur la main-d'œuvre autoprogrammable et organisée autour de réseaux d'ordinateurs. Telles sont apparemment les sources de la croissance de la productivité du travail, donc de la création de richesse, à l'ère de l'information» (Castells $2002: 126$ ). Les exigences conjoncturelles invitent à des pratiques nouvelles, à l'exploitation du potentiel offert par les TIC. C'est justement l'arrivée de ces technologies qui rend la nouvelle économie, malgré ses aléas, ses sursauts, toujours séduisante.

\subsubsection{Les TIC accélèrent le temps et suppriment la distance}

Dans l'économie, dans la société, les TIC agissent sur les dimensions temporelles. Entre correspondants la distance ne compte plus comme auparavant. L'art épistolaire s'étiole et fait place graduellement au courriel. La communication instantanée s'impose. L'accélération du temps suggère une perception renouvelée et une utilisation innovante des différents temps sociaux. La réalité des TIC conduit à appréhender le temps davantage en fonction d'une trame de type «passé-présent-futur», où le temps est «comme un tableau illimité où toute la durée est étalée sous le regard de l'esprit et où tous les événements possibles peuvent être situés par rapport à des points de repère fixes et déterminés. Ce n'est pas mon temps qui est ainsi organisé; c'est le temps tel qu'il est objectivement pensé par tous les hommes d'une même civilisation. Cela seul suffit déjà à faire entrevoir qu'une telle organisation doit être collective» (Durkheim $1985: 14$ ).

Rapportant cette façon de voir à l'ampleur et à l'activité des communautés virtuelles, il apparaît que dans ce cas les TIC contribuent à la dépersonnalisation des rapports sociaux, en raison de la diversité des dimensions temporelles inhérentes à leur fonctionnement. Cet aspect s'éclaire si on s'applique, par exemple, à subdiviser le temps à plusieurs niveaux. Les TIC agissent sur la multiplicité des temps sociaux qui peuvent s'appréhender sous divers angles:

le temps de longue durée et au ralenti (temps du palier écologico-morphologique par exemple); le temps "trompe-l'œil" (temps de la longue durée qui contient des virtualités de crises brusques, comme celui des théocraties charismatiques par exemple; le temps des battements irréguliers entre l'apparition et la disparition des rythmes (temps des rôles sociaux et des attitudes collectives où l'incertitude est due aux heurts, tensions, ...); le temps cyclique (temps des églises, sectes, ...) 
; le temps en retard sur lui-même (temps des symboles collectifs, par exemple, déjà dépassés à peine cristallisés, etc.); le temps de l'altemance entre retard et avenir: c'est le temps des communautés, de la monarchie liée à l'absolutisme éclairé... temps qui oscille entre conservatisme et changement; le temps en avance sur lui-même (temps des effervescences collectives, de l'innovation,...); le temps explosif : c'est le temps des créations, des révolutions... autant de phénomènes qui dissolvent passé et présent dans l'invention d'un avenir immédiatement transcendé (de Terssac et Tremblay 2000:38).

Les TIC accélèrent le temps et rendent les transactions plus fiables, et c'est dans ce sens qu'on peut dire que l'appropriation d'une composante technologique, comme l'ordinateur, a des retombées inestimables (OCDE 2004; Lehr et Lichtenberg $1999: 357$ ). On peut dire que l'usage des technologies accélère le "temps total", qui désigne le "rythme d'une vie qui n'est celle d'aucun individu en particulier, mais à laquelle tous participent» (Durkheim 1985 : 630). Le temps total est «le rythme de la vie collective qui domine et embrasse les rythmes variés de toutes les vies élémentaires dont il résulte; par suite, le temps qui l'exprime domine et embrasse toutes les durées particulières» (Durkheim 1985: 631). Les TIC influencent donc toutes les dimensions de la vie économique et sociale. Elles facilitent la mise en place de repères sociaux nouveaux et encouragent le développement de représentations sociales originales. Elles définissent le temps de travail comme un principal catalyseur de la vie sociale, même si généralement ce temps est «abstrait, imposé de l'extérieur, mesuré et sécable et surtout un temps qui conditionne tous les autres temps sociaux» (de Terssac et Tremblay 2000: 189). Entraînant de nouvelles façons de faire, d'organiser le temps total, le temps du loisir et le temps du travail, les TIC arriveront peut-être à changer une tâche en plaisir, selon ce qu'entrevoyait Edwars Capell au XVIIIe siècle: «Nature has made occupation a necessity; society makes it a duty; habit may make it a pleassure».

\subsubsection{Les TIC : entre l'État et le secteur privé}

Comme pour toute avancée scientifique, il paraît nécessaire que l'État, dépositaire du bien commun, non seulement surveille mais aussi, dans certaines limites, intervienne dans les politiques concernant les TIC. Il est avéré que l'intervention de l'État amène ces technologies à contribuer davantage au développement national, notamment en participant à la renaissance des milieux et territoires en déclin du pays concerné. Les TIC ont également un impact social et culturel que les forces du marché oublient volontiers, mais dont un gouvernement doit tenir 
compte. Ces deux aspects, influence ubiquitaire et impact social sont brièvement évoqués ici, avant de discuter plus en détail des politiques gouvernementales par rapport aux TIC dans la vie économique.

Lehr et Lichtenberg (1999) ont montré que les efforts des gouvernements ont des effets importants quand ils encouragent l'appropriation de composantes technologiques. Chang, Tsai et Lai (1998) ont établi que les délais nécessaires aux gouvernements pour ajuster leurs politiques aux besoins exprimés par les acteurs privés sont déterminants, et ont montré la pertinence, en général, des dépenses gouvernementales à cet égard. Les auteurs concluent à l'existence de liens forts entre les actions du secteur privé et les ajustements des gouvernements (Chang, Tsai et Lai $1998: 644$ ).

Le développement de l'activité économique est crucial pour une nation et les TIC y occupent dorénavant une place centrale. Toutefois, les infrastructures nécessaires à leur implantation et à leur appropriation généralisée exigent des ressources financières considérables, que le secteur privé n'est pas nécessairement en mesure d'assumer. Par ailleurs, l'expérience montre le risque d'abus auxquels les tendances naturelles du marché peuvent donner lieu. On voit donc immédiatement le double rôle de l'État relativement aux TIC dans la vie économique et sociale : le financement et la régulation.

Ainsi, l'appropriation des TIC, fort inégal, doit être vue historiquement comme l'aboutissement d'un parcours original. Les conditions générales qui influencent cette appropriation aident à reconnaître la capacité des TIC à soutenir un développement qui réponde convenablement aux exigences d'une conjoncture donnée. En effet, «as nations continue to differ from each other in corporate structures, in labor relations, in educational systems, in economic policies, and in many other ways, so we should expect them to continue to differ in the technologies they generate and adopt» (Wright 1997:1565). Rendant compte des enjeux liés à l'appropriation des TIC, Wright constate que, généralement, le fonctionnement des TIC dépend de choix stratégiques déterminants, qui influencent profondément l'avenir d'une collectivité, d'une nation.

L'OCDE constate que le fonctionnement des TIC dans la dynamique économique d'une nation peut s'observer sous trois aspects. D'abord selon la contribution du secteur des TIC 
dans l'économie régionale. Ensuite par rapport au changement et à l'accélération que l'introduction des TIC dans l'économie apporte dans les processus de fabrication et de commercialisation de nouveaux produits. De ce point de vue, les TIC sont perçues comme des facteurs de production. Elles sont «des technologies clés pour accélérer le processus d'innovation et raccourcir les cycles, ce qui favorise un lien plus étroit entre les stratégies d'entreprises et l'exécution de la recherche» (OCDE 2000b:52). On peut enfin considérer les retombées de l'usage des TIC comme constituant un indicateur décisif de leur impact sur l'économie régionale. L'une des plus importantes facettes de ces retombées est le fait que ces technologies «s'apparentent aux progrès des connaissances et à l'apparition de nouveaux schémas et de nouvelles formules ou d'innovations organisationnelles susceptibles de bénéficier à tous les acteurs du marché» (OCDE 2000b : 56).

Plusieurs études confirment l'importance de l'intervention gouvernementale dans le développement du monde des affaires. Blumenthal (1998) associe l'appropriation convenable des TIC à l'opportunité des efforts gouvernementaux en la matière. Ces efforts se traduisent par l'appropriation des composantes technologiques par les organismes gouvernementaux qui, par la suite, influencent les pratiques des autres groupes. L'auteur montre aussi que l'influence des entités d'un gouvernement comme consommateurs de technologie a été forte, et que les efforts des acteurs privés à mieux s'approprier les composantes technologiques dépendent de l'efficacité de l'État à rendre la technologie concernée disponible immédiatement sur le marché (Blumenthal $1998: 38$ ).

L'intervention de l'État dans le domaine des TIC se justifie d'abord pour des raisons de coûts. La mise en ouvre des TIC exige en effet des investissements considérables, rarement à la portée d'une entreprise; c'est pourquoi le développement de ce secteur est ordinairement pris en charge par l'État. Pour le développement d'un pays, l'importance de ces investissements soulève des questions politiques; ils sont cependant nécessaires à la promotion de l'innovation et aident à résister aux pressions d'une économie parfois trop centrée sur le court terme. L'intervention des gouvernements devient ainsi une stratégie en vue d'associer la mise en place d'infrastructures technologiques majeures, et l'appropriation des nouvelles technologies, à l'essor des secteurs économique et social. 
Le développement d'une économie axẻe en grande partie sur les TIC invite à reconsidérer les diverses variables qui agissent sur les dynamiques économiques de développement; c'est pourquoi l'on préconise souvent qu'un intérêt particulier soit accordé à «la création de facteurs avancés visant la compétitivité : ressources humaines très qualifiées; système d'innovations technologiques; infrastructures sophistiquées; coopération objective et subjective entre entreprises» (Benko, Lipietz et al. $2000: 291$ ). Selon les auteurs, ces diverses tâches, importantes et coûteuses, nécessitent l'intervention des gouvernements ; elles exigent «un processus qui impliquera beaucoup plus fortement l'État et les institutions locales» (Benko, Lipietz et al. $2000: 291$ ). Quant à Porter, il donne une place importante au secteur privé. Selon lui, les acteurs privés doivent jouer un rôle de premier plan car «economic development is inevitably a collaborative process involving multiple constituencies and it's a process in which the private sector has fundamental roles and a fundamental stake» (Porter 2003a).

Il semble donc que l'évolution continue des TIC révèle un nouvel ordre mondial caractérisé par l'instabilité dans ses dimensions économique, sociale et culturelle. Les enjeux de développement d'un pays, d'un territoire, agissent sur ces dimensions et ouvrent devant les acteurs, individuels et collectifs, des horizons vastes; toutefois l'actuelle division internationale du travail définit la nature de la concurrence entre les firmes, entre les pays et entre les territoires, impose des structures économiques et socioculturelles qui donnent légitimité aux interventions étatiques visant à soutenir le développement des TIC. De nouvelles réalités émergent, qui indiquent à un gouvernement des orientations de nature à améliorer la place que le pays occupe au sein du système international de concurrence. Considérant la division internationale du travail et les compétences en place, Archibugi insiste sur l'importance de bien choisir les axes de développement et de bien cibler les interventions. Il écrit:

in the modern world the division of labour is not such that a single country has a marked advantage in all the high-tech industries. [...] This constrains all industrialized countries, including the large ones, to select the technological areas in which they intend relying on imports. This observation is corroborated by three facts: 1) Technological competence is very different among developed countries. This is reflected both in the sectoral distribution of their innovations and in their international commercial specialization profiles. 2) As stated earlier, the differences in each country's technological competence have increased. 3) 
The place occupied by a country in technological and commercial specialization tends to remain constant over time. Hence, one of the factors allowing a country successfully to exploit its technological competence in foreign markets is the careful selection of the sectors which it chooses to focus on, given its existing competence (Archibugi, Howells et Michie 1999: 259-260).

Importante au niveau international, la concurrence l'est également sur le plan local. Elle conditionne le devenir des forces locales de développement mais présente également des embûches que l'acteur doit affronter s'il espère disposer d'un avantage utile à faire valoir ses propres intérêts. Il y a là des jeux de pouvoir où sont souvent impliquées les TIC, qui brouillent les règles de la concurrence, car elles changent les supports des activités économiques. Ce changement survient parce que les TIC modifient «le pouvoir des acheteurs, le pouvoir des fournisseurs, les menaces de nouveaux entrants, les menaces de produits de substitution et les rivalités entre acteurs existants» (Porter 1999 : 94). L'appropriation des TIC est désormais planétaire. Elle s'inscrit dans un contexte global où le changement s'opère essentiellement par un déplacement des axes de l'activité économique et de ses effets sociaux. Cette observation explique en bonne partie l'exploitation inégale des technologies. Cette inégalité, manifeste aussi bien à l'intérieur d'un pays qu'au niveau international, est un palier essentiel de la nouvelle division internationale du travail.

Les dynamiques économiques actuelles tendent d'ailleurs à accentuer l'inégalité en favorisant la concentration de l'intensité technologique dans certains territoires. Cette concentration touche les outils de communication de même que les moyens de production et de diffusion des produits. La tendance est à l'intégration verticale et horizontale, de façon à pouvoir produire n'importe où, au moindre coût, et à pouvoir échanger sans les contraintes imposées par les frontières et la distance. À cette fin il faut investir d'énormes capitaux et s'assurer de leur mobilité sans entrave. Archibugi rend compte de cette tendance:

it is advantageous for a country to sell its own products in foreign markets and, (...) the advantage becomes even greater if competitiveness is based on sophisticated technological knowledge rather than price. In fact, the former allows the application of profit margins, which are difficult to sustain in areas in which technological barriers to entry are very low. Thus, the preoccupation of political advisors with providing support for industries exporting goods of high technological opportunity seems well founded. It is certainly not by chance that governments provide support for competitiveness of national firms by favouring 
their innovation programmes, so much so that technological policies are increasingly being merged with commercial policies (Archibugi et al. 1999: 258).

Des investissements importants dans les TIC sont donc indispensables si l'on veut garder ou améliorer sa place au sein de la concurrence. La plus grande part de ces investissements devra venir de l'État. Ce faisant, l'État sera gagnant à terme, puisque le seul fait d'implanter les TIC ajoute du poids à l'économie locale, mais surtout fait en sorte que les entreprises du territoire concerné prospèrent et résistent à la concurrence.

Dans la sphère économique, l'interventionnisme gouvernemental prend des dimensions importantes. Les caractéristiques de nos économies dites d'information, largement pénétrées par l'usage de plus en plus important des TIC, incitent les gouvernements à s'impliquer dans la planification. Porter constate que «le rôle de l'État dans l'économie est de développer et de mettre en cuvre un programme d'action économique ou un processus de changement positif, clair et à long terme, qui mobilise l'administration publique, les entreprises, les institutions et les citoyens, en vue d'améliorer (...) l'environnement général des affaires» (Porter 1999 : 256). D'un autre côté, Boyer constate que c'est le rôle d'un gouvernement de veiller à corriger les effets pervers des distorsions du marché. Il écrit :

dans la vision de la nouvelle économie, les crises avaient disparu. Depuis mars 2000, les crises sont partout. Dans une optique régulationniste, une crise intervient lorsque les mécanismes économiques à l'œuvre, impulsés par les institutions héritées du passé, n'arrivent plus à stabiliser les déséquilibres de l'accumulation. C'est ce que montre l'économie américaine actuelle. C'est une crise de régulation puisqu'il faut une intervention publique massive et multiforme pour contrecarrer les risques de récession ou, pire, de dépression. Du coup on retrouve lidée qu'une forme d'intervention publique est constitutive d'une économie de marché. L'erreur serait de confondre une forme d'État avec l'État en général. L'État américain ne manque pas de moyens. Il a une politique fiscale active et une capacité certaine à régler les problèmes que le secteur privé ne peut résoudre à lui seul (Boyer 2001).

Pour Porter, le développement d'une économie moderne « is not something that should be led by government. In fact, it can't be led by government. The more we learn about competition, the more we learn that it has to be a kind of a partnership between government, business, universities, schools, NGOs and other organizations if we're going to actually put in place the environment, the infrastructure required for a healthy economy» (Porter 2003a). En somme, 
les seules forces du marché, «la main invisible» de Adam Smith, ne suffisent pas pour créer une économie saine et durable. La concertation entre acteurs est nécessaire, appuyée par le rôle régulateur de l'État.

De fait, plusieurs mesures dépassent les capacités des entreprises isolées, mais sont devenues si nécessaires que les gouvernements les adoptent pour améliorer la compétitivité des entreprises. Par exemple, un gouvernement peut intervenir «en conseillant les entreprises sur des sujets tels que les lignes de produits, les marchés d'exportation, la technologie et l'organisation du travail. Il accompagne ses recommandations de puissantes mesures financières et fiscales, ainsì que d'un soutien sélectif aux programmes stratégiques de R\&D» (Castells 1998 : 220). Mais dans bien des cas, l'État peut se définir comme

un agent d'intégration s'il s'agit de reconstruire les institutions économiques [...] un coordinateur quand les réseaux locaux ont besoin de soutien des gouvernements locaux et régionaux pour susciter la synergie indispensable à la formation de milieux d'innovation; et messager, chargé de mission, lorsqu'il oriente une économie nationale ou l'ordre économique mondial dans une direction nouvelle, certes inscrite dans la technologie mais non prise en charge par les entreprises (Castells 1998 : 234).

Un État intervient davantage si l'avenir des activités stratégiques est sombre, exigeant des réformes radicales, qui peuvent améliorer le fonctionnement des structures socioéconomiques en place. Dans ce cas, l'État devient le principal développeur : «l'État est développeur lorsqu'il établit comme principe de sa légitimité sa capacité à promouvoir et alimenter le développement, compris comme une combinaison de taux de croissance soutenus et de changements structurels du système économique, tant à l'intérieur que dans sa relation avec l'économie internationale» (Castells $1998: 219$ ).

Il faut en même temps mettre en garde contre toute généralisation qui placerait l'État au centre de toutes les dynamiques économiques et sociales. L'intervention d'un État ne se généralise que dans certains secteurs stratégiques. Ce sont les dynamiques propres à ces secteurs qui incitent les gouvernements à ne pas «se contenter d'organiser des échanges commerciaux, ils doivent aussi fournir le soutien nécessaire au développement technologique et à la formation des ressources humaines, c'est-à-dire aux bases essentielles du fonctionnement de l'économie informationnelle, sachant que les marchés publics (la défense, 
les télécommunications) ainsi que les subventions et les prêts à taux privilégiés du gouvernement (pour la R\&D, la formation, les exportations) aident de manière déterminante les entreprises à s'octroyer une bonne place dans la concurrence globale») (Castells 1998 : 135).

Même si l'État vise à améliorer la compétitivité des entreprises, les mesures qu'il adopte influencent la concurrence entre les firmes et les territoires. 11 peut intervenir de diverses façons. Sans les contrôler, il peut surveiller les médias, en encourageant soit leur convergence ou leur divergence. II peut aussi adopter une réglementation qui impose aux acteurs de développement des comportements et des actions précises: la politique gouvernementale oriente aussi la stratégie, la structure et la rivalité des entreprises à travers la réglementation des marchés des capitaux, la politique fiscale et la législation sur la concurrence Porter 1993 : 140). L'État peut également utiliser l'outil de la subvention, soit pour démarrer une nouvelle entreprise, soit pour en aider une autre à grandir ou à traverser une mauvaise passe. Enfin, un État peut agir au niveau international, au moyen d'ententes de toutes sortes, bilatérales ou multilatérales.

11 reste que, si habiles et généreuses soient-elles, les politiques gouvernementales ne peuvent pas, à elles seules, réguler les effets des TIC sur la société et l'économie. Le cadre institutionnel en place, de même que les initiatives des acteurs locaux et régionaux, jouent un rôle important dans le dynamisme d'un territoire. Par rapport au cadre existant, les acteurs locaux conservent (doivent conserver) une large marge de mancuvre; c'est pourquoi, pour que réussissent les interventions étatiques, s'impose un dialogue préalable entre les intéressés, pour assurer leur compréhension et leur collaboration. Les cadres institutionnels, de même que les initiatives des acteurs, posent donc des limites à la régulation. Il y a là un retour à des principes fondamentaux, qui font que les acteurs s'approprient davantage les TIC au fur et à mesure qu'ils en expérimentent l'utilité et qu'elles leur permettent d'acquérir un avantage concurrentiel.

\subsection{La conjoncture québécoise}

Les scientifiques qui veulent étudier le rôle des TIC dans le développement des territoires du Québec n'ont pas la tâche facile. La difficulté vient principalement de la nouveauté du sujet; 
l'appropriation des TC est en effet récente puisque ses débuts remontent aux années 1980 , mais elle n'a pris des dimensions importantes que vers la fin de la décennie suivante. De plus, l'appropriation des TIC soulève des questions complexes, par la diversité des pratiques qu'elle engendre, les dimensions variées qu'elle donne aux représentations sociales et l'orientation qu'elle impose aux divers secteurs de l'activité humaine.

L'ouvrage récent de Fortin et Sanderson (2004) constitue l'une des premières tentatives de faire le point sur ces questions. Leur publication, sur les TIC et l'identité des collectivités implantées dans les régions québécoises, avait pour objectif de produire des connaissances à jour, complètes et rigoureuses, qui montrent que les TIC, loin de nuire à la cohésion sociale et à l'identité des collectivités, les renforcent. Cet ouvrage mérite donc une attention particulière.

Les spécialistes du développement des régions du Québec trouveront cet ouvrage intéressant, utile et surtout à jour; il présente une réflexion bourrée d'informations et de données précieuses sur les étapes de l'appropriation des TIC au Québec depuis les années 1980 jusqu'à nos jours. Les auteurs font preuve d'une excellente connaissance des enjeux de développement des régions du Québec, notamment du point de vue des dynamiques sociales et communautaires et n'hésitent pas à reconnaître les avantages des TIC pour la vitalité des collectivités périphériques. Ils constatent par ailleurs que les effets des TIC sur le développement des régions du Québec sont assez peu connus chez les spécialistes comme chez les responsables politiques.

Pour les auteurs, il s'agit d'abord de resituer l'appropriation des TIC dans son contexte historique et social. Une meilleure compréhension des effets des TIC sur les dynamiques des territoires québécois repose, en définitive, sur un retour aux divers programmes et initiatives en la matière. Cet exercice montre que les gouvernements fédéral et provincial, de même qu'un grand nombre d'acteurs locaux et régionaux, ont tenté de diverses façons d'influencer les dynamiques de développement de tous les territoires québécois. Les auteurs présentent ensuite un faisceau de définitions, de précisions, d'adaptations et de mises en contexte qui, en plus d'enrichir certains concepts généraux, rappellent les étapes parcourues depuis l'arrivée de l'informatique. C'est à la lumière de cette première mise en situation que les auteurs 
formulent leurs préoccupations, et s'efforcent d'apporter des éléments de réponse aux questions suivantes:

Les collectivités locales et les organismes communautaires se prévalent-ils des possibilités ouvertes par Internet ou disparaissent-ils au profit de groupes affinitaires territoriaux ? quelles identités collectives se construisent et se présentent sur le Web au Québec ? Portent-elles des projets sociaux, des projets démocratiques? Le Web fournit-il une occasion de solidarités, locales et communautaires ? Sert-il à la discussion sur des projets de société ? (Fournier et al. $2004: 8$ )

On voit l'importance de ces questions pour l'avenir des régions du Québec, mais aussi pour qui veut saisir les effets des TIC sur le développement de celles-ci. Pour donner à la tâche que les auteurs se sont fixée l'intérêt qu'elle mérite, ils nous assurent qu'ils vont faire, souvent et tout au long de leur analyse, «un aller-retour entre les données empiriques et les concepts [ et qu'ils récuseront ] tout déterminisme, toute détermination de la technique sur le social» (Fortin et al. 2004: 10).

Par ailleurs, les auteurs ont analysé des données empiriques de grande utilité. Ils ont mis l'accent sur les programmes gouvernementaux, qui ont encouragé l'usage du Web et l'ont mis à la disposition des collectivités. Inscrits dans le cadre des politiques des gouvernements, fédéral et provincial, ces programmes, qui varient beaucoup au fil des années, ont eu des conséquences importantes sur l'appropriation du Web. Ils ont facilité l'accès des communautés à une information stratégique. Les organismes locaux et régionaux ont repris les orientations gouvernementales et les ont adaptées aux conjonctures en place. Les auteurs montrent en définitive que, dans le contexte québécois, l'intervention des gouvernements a été utile pour généraliser l'usage du Web. Ils estiment que, dans les régions du Québec, Internet permet «d'entamer le dialogue, la discussion, le débat sur des projets sociaux, voire l'élaboration de contre-projet» (p.34). C'est un «outil décentralisé (qui) facilite la création de réseaux» (p. 34). Internet «peut à la fois servir d'espace de socialisation et de formation d'identités individuelles, bref de lieu de dialogue et d'intersubjectivité, de support pour des productions culturelles et de lieu de délibération collective» (p. 145).

De plus, le Web met en place un espace social qui «n'est pas l'univers des identités "particularistes" de personnes qui réussiraient "enfin" à dépasser les contraintes de temps et 
d'espace et à en rejoindre d'autres aux intérêts similaires» (p. 137). L'arrivée de l'Internet et les effets des TIC font que l'espace ne se limite plus à 'l'ancrage géographique", à la "localisation", il est enrichi par de nouvelles dimensions ajoutées par Internet, qui permet désormais de parler de "Cyberespace", lequel «est approprié par plusieurs acteurs» (p. 141).

La nature de cet espace reflète la complexité de l'ancrage géographique (p. 137) et des usages que l'on fait de l'Internet. Malgré cette complexité «le cyberespace s'est [...] révélé un formidable lieu de définition identitaire collective, une place publique où la parole s'énonce de toutes parts, et dirait-on parfois, sans même chercher à circuler. Le cyberespace, s'il semble a priori relever de la globalisation, se développe aussi en s'appuyant sur des identités locales. Il sert autant à créer le global qu'à renforcer le local. Ces aspects du cyberespace sont ceux qui nous semblent les plus prometteurs pour l'avenir, c'est là où on peut observer à chaud l'expression et l'invention de nouvelles identités collectives, porteuses de projets (Fortin et al. $2004: 146$ ). L'appropriation des TIC et l'usage grandissant de l'Internet font que «'identité locale continue de s'affirmer et profite même du cyberespace pour le faire (p. 141). «La fonction identitaire [...] est assez explicite» (p. 143).

Malgré l'attrait qu'exercent l'Internet et l'influence du Web sur le devenir des régions du Québec, les auteurs sont conscients de certaines contraintes, dont il faut tenir compte, car «le bilan n'est pas que positif » (p. 142). Les usages et les objectifs poursuivis par les acteurs incitent à la prudence (p. 143), car une nouvelle dynamique de développement se met en place. À travers toutes les zones grises, «se met en place, se structure et se renforce une identité locale et régionale: histoire, géographie, vie artistique et culturelle, activités communautaires " (p. 143). Mais « si ce potentiel existe bel et bien, il ne se réalise pas automatiquement» [p. 144].

L'intérêt de cette réflexion vient surtout de ce qu'elle repose principalement sur des constatations relatives aux régions du Québec. L'essentiel, semble-t-il, c'est que l'appropriation des TIC, en activant les dispositions sociales et culturelles des régions périphériques, renforce les identités locales. À cela nous croyons cependant devoir apporter une réserve. 11 paraît en effet hasardeux d'avancer que l'appropriation des TIC renforce les identités locales en se basant uniquement sur leurs effets sur la vie sociale et culturelle, sur la diversité des programmes et d'initiatives mises en place par les différents paliers 
gouvernementaux. Il serait utile d'aller plus loin, pour comprendre comment, dans les territoires québécois, l'usage de plus en plus important des TIC influence les conditions économiques des collectivités concernées.

Force est de constater que les auteurs sont allés à contre-courant d'opinions voulant que les TIC "détruisent les régions", ou tout au moins, que l'appropriation des TIC n'ajoute qu'une valeur minime aux activités des organisations et aux dynamiques des régions. Cette thèse a été souvent reprise au Québec et ailleurs par des scientifiques québécois. Par exemple, Robillard (2004) considère que l'appropriation des TIC n'apporte rien de nouveau, et qu'elle n'a qu'une influence limitée sur la vie au travail, sur les méthodes de gestion de l'information et des connaissances individuelles et organisationnelles, ainsi que sur la stratégie des organisations. Certes, les TIC ne sont pas une panacée aux difficultẻs réelles que rencontrent les acteurs; mais leurs nier pratiquement toute influence sur l'activité économique parait excessif.

\section{Conclusion}

Ce premier chapitre présente un aperçu des problématiques et des hypothèses qui ont cours depuis longtemps autour des changements technologiques. La littérature qui a servi de point de départ à notre réflexion montre le cheminement des idées depuis les années 1950 jusqu'à nos jours. Sont ainsi mises en lumière les constatations et les analyses des chercheurs, quant aux effets de l'innovation technologique en général, et quant à l'introduction plus récente des TIC dans la plupart des secteurs d'activité. L'examen de ces questions montre que le changement technologique met en action des processus indistincts d'adaptation individuelle et organisationnelle.

La compréhension des répercussions directes, mais surtout médiates, d'un changement technologique a historiquement été une tâche complexe (Wright 1997: 1562). En effet, des changements technologiques s'observent partout, mais n'interviennent pas toujours de la même façon. Leurs effets sur le déroulement des activités économiques varient selon les circonstances, et selon l'usage social que l'on fait des composantes technologiques. Cet usage donne des indications sur les dynamiques économiques et sociales en place. Mais pour bien saisir les effets du changement technologique, il faut connaître le contexte préalable au 
changement. $\mathrm{Ce}$ que résume bien la pensée suivante: "The broader lesson is that if economics really wants to take technology seriously, economics will have to become a more historical discipline» (Wright 1997: 1565). Dans tous les cas, l'Internet et les TIC sont généralement à l'origine des changements qui touchent la société contemporaine : «Internet and related information and communications technologies are central to the changes that are taking place» (Malecki et al. 2004: 1).

Révélateur fidèle des changements qui marquent le développement des sociétés et des économies, le débat suscité par l'arrivée massive des technologies de pointe témoigne de la complexité des phénomènes en cours dans diverses régions de la planète. C'est pourquoi le chercheur qui traite de l'influence de ces technologies dispose toujours d'arguments pour justifier son point de vue, qu'il soit un fervent des apports technologiques à l'économie et à la société, ou qu'il y voie un facteur déstabilisateur des équilibres traditionnels.

La recension de la littérature montre que les effets des technologies sur la société et sur les pratiques économiques doivent être appréhendés en fonction des conjonctures en place qui, selon leur propre logique, s'ajustent à l'arrivée des technologies de pointe. Loin d'être l'aboutissement d'initiatives isolées, l'appropriation des technologies s'inscrit dans un cheminement socio-historique particulier, à l'image des stratégies collectives mises en place par les principaux acteurs partageant un même espace géographique, un territoire. Ainsi l'appropriation des technologies soutient le progrès des conditions matérielles des sociétés et dicte, directement ou non, l'ensemble des choix offerts aux acteurs en place.

La transformation des conditions matérielles dans une direction et non dans une autre dépend largement des aptitudes des acteurs de développement, de leurs intérêts individuels et collectifs. Les effets des technologies doivent donc être compris à la lumière des facteurs culturels dominants et du fait que la production de services s'inscrit désormais dans un contexte où le réseautage, l'information et son économie, prennent de plus en plus d'importance. 


\section{ChAPITRE 2}

\section{L'INFORMATION, SËVE DES RÉSEAUX D'ENTREPRISES}

Devenus pratiquement indispensables, les réseaux d'entreprises offrent une attrayante réponse au besoin de placer les pratiques entrepreneuriales dans le champ exubérant de l'économie de l'information, elle-même portée par l'usage croissant des TIC. Bien que ces questions aient suscité ces dernières années un regain d'intérêt chez les chercheurs, la compréhension des rapports entre technologies de communication, information et réseaux d'entreprises, demeure imparfaite, de même que les mécanismes par lesquels l'offre et la demande d'information agissent sur le fonctionnement et le développement des réseaux. Ce chapitre examine comment et à quel point les TIC, et l'information qu'elles véhiculent, influent sur les réseaux d'entreprises, et comment ceux-ci participent à la diffusion des connaissances.

C'est d'abord à l'information qu'il convient de s'intéresser, pour en mieux voir la nature, les facettes variées et l'influence, dans le cadre général où elle se propage. Les réseaux d'entreprises sont ensuite considérés dans leur diversité et comme lieux de production et de circulation de l'information. Éventuellement, s'ils fonctionnent bien, ces réseaux deviennent des lieux d'apprentissage et d'innovation propices à la croissance de l'entreprise et au développement du territoire. 


\subsection{L'information}

Notion polysémique s'il en est, l'information s'entend différemment selon le contexte, car elle est à la fois l'acte et son contenu. Il y a dans l'acte d'information une connotation de réciprocité: soit l'action d'informer ou le fait de s'informer : un informateur et un informé. Par ailleurs l'objet de cet acte, son contenu, qui est un renseignement, transmis puis acquis par le récepteur, est également dit information. Bien que sémantiquement distinctes, ces notions sont dans la pratique inséparables. Dans le contexte du présent travail, le mot est généralement pris dans son acception de contenu en rapport avec l'entreprise et le monde des affaires.

Notre époque est caractérisée par la production pléthorique d'information en tout genre : utile, banale, triviale, etc. L'information toutefois ne se confond ni avec la connaissance, ni avec le savoir. La connaissance succède à l'information; quant au savoir, il est relativement rare. L'information est un ensemble de renseignements obtenus sur un être, un fait, etc. Elle mène à la connaissance quand les renseignements sont triés et ordonnés selon une certaine hiérarchie pour devenir une représentation. L'information est avant tout «ce qui forme ou transforme une représentation dans la relation qui lie un système à son environnement» (Mayère 1990: 56). Selon Cooke et al. «Information is relatively globally mobile [...] Knowledge is remarkably spatially rooted» (Cooke et al. 2000:12). Vient ensuite le savoir. Claire perception d'un fait, d'un objet, le savoir est un ensemble de connaissances acquises par l'étude, l'expérience, et assimilées en un tout cohérent. Il s'ensuit que, pour l'entreprise, si indispensable que soit l'information, son utilité se mesure par sa pertinence à l'action et au projet envisagé. Dans la masse d'information disponible, il faut donc choisir, distinguer l'information utile de celle qui ne l'est pas et, en principe, disposer d'un minimum de connaissances préalables.

\subsubsection{Types d'information}

L'analyse de la littérature permet de distinguer plusieurs types d'information. Chaque type réfère non seulement à une situation où l'acteur confronte un ensemble de contraintes liées aux dynamiques économiques et sociales de développement de son environnement, mais surtout aux stratégies et outils qu'il choisit afin d'obvier à ces contraintes. Ainsi, on peut parler de l'information de veille, qui enrichit la connaissance de l'acteur sur son 
environnement matériel et professionnel, et atténue son incertitude. De même, un acteur peut utiliser une information pour suivre l'évolution d'une situation ou d'une question qui le préoccupe. Dans certaines situations, l'acteur s'informe afin de préparer à l'avance une riposte à une crise ou à une conjoncture pouvant menacer le développement de ses affaires; l'information sert donc à anticiper les faits. On peut aussi penser à l'information qui guide un acteur, et aide à orienter son action : c'est l'information stratégique.

Cette classification permet de distinguer l'information générale de l'information spécifique. Contrairement à celle-ci, l'information générale est accessible à tout le monde, aucun obstacle ne l'empêche de rejoindre un public. On doit toutefois en connaitre les limites, car elle s'accommode d'un contenu hétéroclite, voire d'éléments contradictoires. Par contre, l'information spécifique s'adresse à un individu ou à un groupe déterminés, avec un contenu particulier à l'objectif poursuivi. C'est pourquoi cette information coûte cher et peut demeurer inaccessible à certains acteurs. Si cette classification s'accepte facilement dans l'absolu, il demeure difficile, en pratique, de différencier les types d'information. C'est pour cette raison que la littérature spécialisée met davantage l'accent sur l'information concernant le monde des affaires.

Particulièrement depuis l'avènement des TIC, l'information circule le plus souvent presque librement et indépendamment de la volonté de ses auteurs, et elle agit comme une sorte de cadre commun, constamment changeant, où les acteurs tendent à inscrire leurs pratiques. Mayère associe à l'information trois caractéristiques. D'abord que «l'information n'existe pas en soi mais qu'elle devient information dans un processus qui engage activement le système qui l'acquiert» (Mayère 1990: 56). Elle est de plus «relative au système dans sa signification et son usage»; et enfin à toute information est attachée une forme particulière d'incertitude.

\subsubsection{Sources ef immatérialité de l'information}

L'information accessible aux entreprises provient de sources diverses, et on peut la classer de plusieurs façons. On peut par exemple parler de l'information générale versus l'information spécifique. De même, l'information peut provenir de sources publiques ou privées. Les sources publiques réfèrent principalement aux communications émanant des États et des gouvernements, ainsi que d'une variété d'organismes publics et semi-publics. En principe, ces 
communications ont plutôt un caractère général, sont factuelles et fiables. Dans le cas des sources privées, on trouve une variété d'intervenants engagés à divers titres dans la production, le traitement et la circulation de l'information. Les communications qui en émanent visent souvent des segments particuliers d'acteurs économiques ou de consommateurs et sont ordinairement de qualité, quoique à l'occasion il soit difficile de départager entre l'information pure et la publicité. Bref, toute information est plus ou moins ciblée pour une certaine audience, et il revient aux acteurs des divers champs d'activité de l'interpréter en fonction des incertitudes qu'ils confrontent et des besoins de leur organisation.

Entre l'immatérialité de l'information et son encastrement social se situent les aléas de son économie et son rôle comme source d'innovation et de dynamique territoriale. Que les acteurs de développement s'en saisissent ou non, l'information est un produit social, parfois posé hors du temps de sa production et de sa circulation, mais toujours ancré dans un temps qui est celui de l'action et des réactions que l'information suscite. La circulation et l'appropriation de l'information dépendent en définitive non seulement des besoins ressentis par les organisations et les acteurs d'un territoire, mais de leur habileté à établir leurs stratégies de développement tout en participant à la promotion du cadre commun. En outre, «la circulation de l'information (...) est facilitée par la proximité, les liens technologiques ou de clientfournisseur et l'existence de relations personnelles et sociales répétitives» (Porter 1999 : 94 ).

L'immatérialité de l'information en fait un catalyseur de l'action économique et sociale, un rôle dont on ne peut que pressentir limportance, et qui dépend de la façon dont les acteurs font circuler cette information et la reçoivent. L'information est donc un bien multidimensionnel. Mais il ne faut pas réduire son importance à la contribution de sa nature immatérielle. Bouchy écrit que les «systèmes (d'information et d'informatique) sont des objets plus ou moins immatériels. Personne n'a jamais vu un système d'information et ce système n'est visible qu'à partir de ses représentations : documentation, modèle, etc. Cela pose le problème de l'abstraction qui, seule, permet d'imaginer l'objet à partir de ses plans, de ses modèles, et de la conformité de ses représentations par rapport à l'objet lui-même» (Bouchy $1994: 27$ ). L'immatérialité ne peut avoir de sens que dans la mesure où le monde matériel, avec ses outils de haute technologie, donne un sens à l'information. Les objets à base de haute technologie deviennent donc le support matériel qui rend possible la tâche de 
qui veut manipuler l'information. Ils remettent en question les supports traditionnels d'échange et les complètent. En fait,

bien que tout échange d'information ne puisse pas être supporté par les TIC, la diffusion de ces techniques favorise le développement des échanges informationnels. Deux éléments en sont la clé. Les techniques de télécommunications permettent de distribuer l'information de manière rapide, fiable et confidentielle. La programmabilité des TIC permet d'incorporer à un support physique, reproductible et appropriable, une ressource auparavant difficilement transférable : le savoir-faire (Brousseau $1993: 207$ ).

\subsubsection{Rôles et effets de l'information}

L'un des rôles de l'information est de consolider le tissu organisationnel d'une institution. $\grave{A}$ cette fin, l'information circulante doit pouvoir aider les acteurs à modeler leurs stratégies selon la conjoncture du moment. Dans le cadre local de l'entreprise, les TIC donnent à l'information une plus-value en facilitant sa circulation entre les intervenants et son utilisation dans les pratiques quotidiennes.

C'est d'abord à partir de sa dimension locale, donc économique et sociale, que l'information s'implante au centre de tous les systèmes techniques, économiques et socioculturels. L'information y subit des transformations, des adaptations, des manipulations accélérées. Elle dépend ainsi de la continuité de ces systèmes dans le temps. Or ces derniers évoluent plus rapidement qu'auparavant, entraînant parfois des changements radicaux. Zimmermann s'en explique ainsi :

placer l'information au centre des systèmes qu'elle forme la déclare fille naturelle de la complexité des grands systèmes économiques et sociaux dans lesquels elle se forme et agit. Cet emplacement la définit comme le moteur de l'action, mais aussi comme source d'éventuelle lèpre pouvant attaquer une économie ou une société. II est évident que la nature ou la qualité de cette information se rallie souvent à des conditions économiques et sociales ne dépendant aucunement d'elle ni de ses enjeux. Ces conditions sont le fruit des parcours socio-historiques propres à chaque territoire et à chaque dynamique de développement. Les acteurs qui se parent de l'information inscrivent automatiquement leur action dans un monde dominé par l'immatériel. C'est pourquoi "la montée de l'immatériel" au sein de l'activité industrielle doit s'analyser à travers l'intervention directe de biens de nature informationnelle et cognitive au sein même du système technique. (...). Dès lors, de tels objets soft deviennent le centre de jeux 
concurrentiels sur des marchés et, de par leur nature particulière, (nous) interrogent (Zimmermann 1995: 181).

Il existe donc une relation étroite entre l'information et les dynamiques économiques de développement. Cette relation est certes aidée par le développement spectaculaire des TIC. Effectivement, Jorgenson et Stiroh (1999) ont démontré que l'information constitue un capital qui peut avoir la même importance que le capital financier et le capital humain. Afin de comprendre comment circule l'information, ils se sont intéressés à l'ordinateur et à son utilisation dans les entreprises et dans les ménages. Et leurs résultats montrent qu'il existe une relation significative entre l'usage d'un ordinateur et l'intégration de l'économie de l'information.

En fait, l'arrivée en masse des ordinateurs, et de l'information qu'ils déversent aux acteurs économiques, foumit aux entreprises la possibilité de maximiser leurs profits, en même temps que les consommateurs se familiarisent avec l'arrivée sur le marché de marchandises et services nouveaux; l'ordinateur servant d'intermédiaire entre ces deux mondes. Cela est d'autant plus important que l'utilisation d'un équipement informatique facilite et accélère bien des travaux, laissant aux utilisateurs le loisir de concentrer leur énergie sur d'autres tâches. Les auteurs concluent qu'un recours massif aux composantes technologiques vectrices d'information, comme l'ordinateur, constitue un moteur de croissance et de développement (Jorgenson et Stiroh $1999: 110$ ).

II apparaît par ailleurs que les TIC, au centre de l'évolution présente et annonçant l'économie dématérialisée, sont à façonner un nouveau cadre social. On assiste à «la marchandisation de l'information (qui) impose une évolution des marchés de l'information et des connaissances dépendantes de l'être humain» (Mayère 1997: 31). C'est pourquoi Bontis et Choo (2002) proposent de traiter de l'information en fonction de l'impulsion qu'elle donne au développement des connaissances. Ils notent que l'information est créatrice de connaissance et que les rapports entre les deux sont à la base des sciences de l'information.

Plusieurs études ont traité des changements entrainés par l'importance accordée à l'information dans l'économie et la société. Parmi d'autres pratiques nouvelles, l'avènement des bibliothèques numériques transforme profondément le classement, l'organisation, la recherche et l'utilisation de l'information. Borgman (2002) s'est intéressé aux incidences de 
cette transformation. Il remarque que les institutions dont les activités sont concentrées sur la gestion de l'information acquièrent une importance grandissante dans nos sociétés. Traitant des contingences propres à ces institutions, il considère que leur problème majeur concerne l'infrastructure nécessaire à la gestion convenable de l'information ; l'apparition du concept de «cyberinfrastructure» est un indicateur de cette évolution. Borgman (2000) estime que le grand objectif, pour toute institution ou organisation, est de pouvoir se tailler une place dans l'infrastructure mondiale d'information, ce système d'interconnexion de réseaux informatiques et de diverses formes de technologies de l'information à travers la planète.

Les travaux de Agre reflètent une autre dimension de l'information, celle qui touche la société et le cours de la culture en place. Ses travaux font voir l'importance des technologies de l'information en général, et de l'Internet en particulier. Ébranlant l'ordre établi, ces technologies n'ont pas créé un nouvel ordre; elles ont plutôt agi sur les anciennes structures qu'elles ont modifiées et ont largement influencé les processus sociaux et politiques qu'elles ont intégrés (Agre 2003). Ainsi se sont imposées des pratiques nouvelles apparues grâce à l'appropriation croissante des TIC et à l'avantage qui s'en est suivi dans nombre de secteurs d'activité.

De leur côté, de la Mothe et Paquet ont étudié, des points de vue économique, social et statistique, l'effet d'une utilisation novatrice de l'information. Dans une économie d'information, leur analyse démontre l'existence d'un rapport coût-avantage positif qui peut résulter, pour la croissance économique et le développement, de la disponibilité de données, d'information, et de la connaissance codifiée des dynamiques de linnovation. Selon les auteurs, l'information traverse divers cycles avant d'avoir un effet tangible. Il y a en réalité une grande distance entre la forme cognitive de l'information et sa répercussion sur l'environnement économique qui la produit (et la reçoit) (de la Mothe et Paquet 2000: 25). Pour ces auteurs, l'information produit des effets dont l'importance dépend à la fois de la complexité du système socioculturel existant et de la flexibilité organisationnelle :

Quite clearly, informational innovations increase dramatically the range of possible behavior of consumers and the range of potential business strategies of firms. But the parallel forces that make possible globalization and centralization of decision-making, also, at the same time, allow moves toward localized 
communities of practice and decision-making. It is difficult to determine which forces will prevail hic et nunc (de la Mothe et Paquet 2000: 25).

Ainsi le devenir de l'innovation, par rapport à l'information ambiante dépend de l'habileté des acteurs à mettre cette information au service des dynamiques économiques et socioculturelles de développement d'un territoire.

\subsubsection{L'information comme source de changement}

Pour une organisation, pour un territoire, on peut considérer la valeur de l'information selon trois critères. Encastrée dans le cadre commun qui la génère, elle répond à des besoins bien définis. De plus I'usage qu'en font les acteurs lui donne une valeur et la distingue des autres biens et services. Enfin elle agit directement sur les dynamiques de développement. L'information devient utile, voire stratégique, lorsque corrélée à toute action qui permet à un acteur non seulement de mieux se positionner dans le système entrepreneurial, mais surtout lorsqu'elle lui facilite le passage d'une situation vécue à une autre souhaitée (Mayère 1990 : 50). C'est ainsi que «des "marchandises" informationnelles offrent de nouveaux moyens pour réduire les coûts, améliorer la qualité des produits, faciliter un contrôle flexible, favoriser l'adaptation aux conditions du marché, renforcer l'innovation organisationnelle et technique» (Mayère 1997: 30).

Disséminée par les TIC, l'information acquiert une puissance accrue, qui intensifie sa pénétration et son influence; elle devient porteuse d'un nouvel ordre économique et social où sont remises en question les pratiques des producteurs et des consommateurs. Cette remise en question est accélérée par l'augmentation continue de la capacité des acteurs à stocker, traiter et transmettre de l'information. Ce pouvoir influe nécessairement sur les dynamiques des marchés et sur la division du travail. Porter constate que dans un environnement acquis aux TIC, l'information agit «sur le pouvoir des acteurs, le pouvoir des fournisseurs, les menaces de nouveaux entrants, les menaces de produits de substitution et les rivalités entre acteurs existants» (Porter 1999 : 94). Tissant des liens, à la fois forts et faibles, entre différentes catégories d'acteurs, les TIC, en décuplant la puissance de l'information, en ont fait un agent de transformation dans l'entreprise et au-delà. Porter précise qu'elle 
modifie la structure des industries et donc les règles de la concurrence; elle crée des avantages concurrentiels en apportant aux entreprises de nouvelles manières de faire mieux que leurs rivales; elle fait naître des entreprises entièrement nouvelles, souvent issues d'activités existantes (Porter 1999 : 84).

De son côté, Choo (1999) a montré que les organisations qui s'approprient convenablement l'information deviennent également plus aptes à en faire le traitement. II confronte divers points de vue concernant la relation entre l'information et les connaissances d'une part, et de $1^{\prime}$ autre, les divers processus qui y sont associés. Selon lui, cette confrontation est une démarche nécessaire pour mieux comprendre le fonctionnement d'une organisation dans une ère dominée par l'information et son économie. La gestion et le développement de la connaissance dans une organisation dépendent de la capacité des dirigeants à mieux intégrer l'ordre informationnel. L'auteur présente un modèle original, qui permet de voir comment différentes organisations recherchent et traitent l'information. Celle-ci s'inscrit ainsi dans un faisceau complexe d'emplois qui la rendent nécessaire à la réalisation de plusieurs tâches quotidiennes. L'information peut être choisie, transformée et insérée dans divers processus. Par sa contribution à l'accroissement des connaissances, elle fournit un éclairage indispensable à la prise de décision. C'est donc selon ces différents niveaux que l'information participe à la transformation des organisations, qui s'adaptent ainsi aux exigences du nouvel ordre informationnel (Choo 1999).

\subsection{Les réseaux : caractéristiques et avantages}

On estime généralement que les différends, les contestations entre entreprises sont plus rudes quand, dans une région, n'existe pas un lieu de rencontre, d'échange de vues sans formalité, où les acteurs de l'économie apprennent d'abord à se connaître, et au besoin, à s'entraider. Isolée, une PME est plus vulnérable. Tandis que le groupement des entreprises d'un territoire tend à minimiser les désaccords et encourage plutôt la coopération, selon l'adage que l'union fait la force. L'entreprise s'engage donc dans un réseau, et en profite selon la place qu'elle occupe au sein des systèmes économiques et socioculturels en place. Les écoles de pensée définissent le réseau d'entreprises selon des perspectives différentes. Dans la littérature du GREMI, le réseau d'entreprises est vu comme 
un ensemble formé de liens sélectionnés et explicites avec des partenaires préférentiels inscrits dans la perspective des relations de marché d'une entreprise et de sa recherche de ressources complémentaires ayant comme objectif principal la diminution de l'incertitude. Cette définition est complétée par celle d'économie réticulaire: une économie réticulaire est celle dans laquelle le foyer dominant de la constitution de la valeur consiste dans des architectures flexibles et inter-reliées qui permettent la gestion de relations individuelles en tant qu'ensemble (Maillat, Quévit et Senn 1993 : 12).

Les écrits du GREMI associent les dynamiques d'un milieu innovateur au développement des réseaux d'innovation. Ce sont donc les caractéristiques de ces dynamiques qui déterminent l'émergence des pratiques de coopération et de coordination entre les acteurs. Le GREM présente un réseau d'innovation comme une réalité pluridimensionnelle que l'on peut caractériser de la manière suivante: 1 une dimension organisationnelle; 2 . une dimension temporelle; 3 . une dimension cognitive ; 4. une dimension normative; 5 . une dimension territoriale (Maillat, Quévit et Senn $1993: 14$ ).

Ratti définit les réseaux d'entreprises comme «un ensemble de coopérations explicites (formelles) entre entreprises qui se cherchent mutuellement en donnant lieu à un processus d'apprentissage collectif» (Ratti 1992 : 56). A la source de cet apprentissage, l'information investit le réseau de nouvelles dimensions, aide les acteurs à faire face aux inévitables zones d'incertitude. Ces zones prennent forme aussi bien dans les champs économiques que sociaux. On peut distinguer cinq types d'incertitude :

\begin{abstract}
"information gap" dû à la complexité, à l'étendue et au coût de la collecte d'information; "'assessment gap" ou "lacune d'évaluation", liée à la difficulté de déterminer ex-ante ce qui se cache sous la "peau" de certains inputs; 'competence gap', ou défaillance (ex. : due à des procédures techniques mal connues); "competence-decision gap", c.àd. le risque - face à un futur peu prévisible - de choisir la fausse technologie ou une technologie inférieure; "control gap" ou l'incertitude liée au contrôle imparfait d'un contexte dynamique de décision (Ratti 1992: 55).
\end{abstract}

Ratti constate que la gestion des situations d'incertitude «passe par des mesures prises à l'intérieur même de l'entreprise, ou par le recours au marché, mais avec des coûts de transaction élevés, ou bien (...) par des formules intermédiaires (hiérarchie, accords d'alliances)» (Ratti 1992: 55). I1 faut préciser que l'incertitude inhérente à toute entreprise est exacerbée par l'introduction des TIC, en sorte que nos sociétés se distinguent par 
«linstabilité, l'insécurité, linégalité et l'exclusion sociales qui accompagnent la créativité, linnovation, la productivité et la création de richesse dans ces premiers pas du "monde Internet"'》(Castells 2001: 13). Ce qui permet de voir un réseau comme un système décomposable, qui est plus que la somme de ses composants, lesquels agissant l'un sur l'autre, activent l'interaction des membres de réseaux (Cooke et Morgan 1998) et approfondissent les effets de synergie.

L'influence croissante des TIC dans le processus de réseautage donne à la notion de réseau une signification élargie, qui comprend à la fois des aspects particuliers et d'autres plus généraux. Les aspects particuliers sont des caractéristiques propres à toute organisation, à un milieu, à un territoire ou à une structure socioculturelle. Les aspects généraux désignent l'ensemble des normes et des valeurs, enracinées dans un terroir socioculturel, qui encouragent diverses formes de coopération et de coordination des actions. Ces derniers aspects font que «les gens suivent automatiquement et inconditionnellement les coutumes, les habitudes ou les normes» (Porter 1999: 84), et cherchent à se soustraire aux contraintes résultant principalement de l'incertitude. Les réseaux d'entreprises intègrent ces normes et ces valeurs, empruntées au cadre socioculturel, pour en imprégner les pratiques entrepreneuriales.

Par ailleurs, Callon voit le réseau d'entreprises comme une méta-organisation, qui se fonde sur les rapports se développant entre «un ensemble d'entités humaines et non humaines individuelles ou collectives (définies par leur rôle, leur identité, leur programme,...) et des relations dans lesquelles elles entrent» (Callon $2001: 204$ ).

Pionnier de la sociologie de l'innovation, que l'on appelle aussi sociologie des réseaux techno-économiques, l'auteur avait présenté le réseau comme une organisation qui s'inscrit dans un cadre agrandi, constitué de divers éléments impliqués dans le monde de la production. Des compromis négociés entre ces éléments donnent au réseau d'entreprises ses qualités et valident sa structuration dans une sorte d'agencement et d'équilibre. Le fait nouveau est de considérer le réseau d'entreprises en rapport avec les technologies d'information et de communication, qui multiplient les interactions entre les acteurs d'un même milieu et même entre ceux que séparent de longues distances. En fait, l'arrivée en masse des TIC change les caractéristiques d'un réseau d'entreprises. Castells écrit : 
un réseau est un ensemble de nouds interconnectés. Ce type d'organisation humaine qui remonte à la nuit des temps entame une nouvelle vie à notre époque avec les réseaux de linformation, dont la force motrice est Internet. Les réseaux sont des modes d'organisation aux avantages extraordinaires, parce qu'ils sont naturellement flexibles et adaptables, qualités essentielles pour survivre et prospérer dans un environnement qui change vite. Voilà pourquoi les réseaux proliferent et ont toujours existé dans tous les domaines de l'économie et de la société: ils se révèlent plus compétitifs et plus efficaces que les entreprises hiérarchisées et les bureaucraties centralisées (Castells $2001: 9$ ).

Aux caractéristiques habituelles des réseaux d'entreprises, il faut donc ajouter des dimensions nouvelles, extensibles, qui augmentent leur flexibilité devant des réalités diverses. Ces réseaux, qui ont effectivement envahi toutes les régions de la planète, intensifient les activités de coopération et de coordination entre des acteurs de développement stimulés par la conjoncture actuelle. Pour plusieurs, $c^{\prime}$ est une condition de survie : «si nous ne nous occupons pas des réseaux, les réseaux, eux, s'occuperont de nous. Qui veut vivre en société à cette époque et en ce lieu sera nécessairement confronté à la société en réseaux. Car nous sommes bel et bien entrés dans la galaxie Internet» (Castells 2001 : 342). Nous sommes entrés dans l'ère qu'ils sont à façonner: «our age is the network age, and hence it is plausible that entrepreneurs try to extract a maximum performance from participation in network constellations, both physical and virtual (Malecki et al. 2004: 1).

\subsubsection{Réseau ou alliance}

Les réseaux d'entreprises existent en plusieurs catégories et les spécialistes les décrivent de diverses façons. Il faut distinguer les réseaux d'entreprises des alliances entre entreprises. Ces alliances, comme les décrit Porter, «sont des accords à long terme entre deux ou plusieurs entreprises : elles vont au-delà du niveau habituel de transactions commerciales, mais ce ne sont pas des fusions (...) Le terme "alliance" va recouvrir toute une variété d'ententes interentreprises... $\gg$ (Porter $1993: 72$ ).

Bien que les avantages des réseaux aient été abondamment démontrés, pour autant ils n'assurent pas automatiquement la croissance d'une firme. Le réseau peut offrir une variété d'appuis mais il ne peut suppléer à l'impéritie. Par ailleurs d'autres facteurs ressortissant aux relations humaines, à la conjoncture ou à la concurrence, peuvent miner l'efficacité d'un réseau. 
Il arrive qu'un entrepreneur choisisse de faire alliance avec une ou plusieurs entreprises, plutôt que d'adhérer à un réseau. Il s'agit le plus souvent d'arrangements ponctuels, à but précis, entre organisations travaillant en complémentarité. Ces alliances peuvent contribuer à concrétiser une idée en innovation, mais arrivent rarement à assurer la croissance de l'entreprise quand il devient nécessaire d'injecter un capital important. Niosi décrit cette situation :

all networks and alliances are not conducive to growth. This result qualifies the abundant network explanations of the dynamics of biotechnology. Interaction with research universities and government laboratories is almost always present in biotechnology start-ups. But success (as measured by growth) is linked to the financial support of venture capital and large corporate partners (Niosi 2001: 749).

La structure interne d'un réseau d'entreprises est habituellement influencée par l'intérêt accordé, dans un territoire, aux mécanismes de l'offre et de la demande d'information, ce qui définit en même temps les paramètres d'une relation tendant à la réciprocité. Ensemble, ces mécanismes et paramètres influencent les interactions entre l'individu et ses pairs. Le réseau d'entreprises est un lieu où l'information est avant tout une relation, dont l'influence sur l'action et sur les mécanismes du marché ne s'opère pas spontanément, mais selon une logique calculée et toujours inscrite dans un espace social délimité : «l'aspect social est un espace non naturel puisque intégralement organisé- sinon même produit- par des conventions. Ces conventions, à leur tour, sont pensées sous la forme pragmatique du compromis» (Miguelez $2001: 295$ ).

\subsubsection{Réseaux et information}

La présence de réseaux d'entreprises crée une certaine interdépendance, observable entre le domaine social et les dynamiques économiques d'un territoire, ce qui favorise la circulation rapide et efficace de l'information entre l'entreprise et son milieu. Cette interdépendance peut s'expliquer par le pouvoir de l'information à créer des ponts entre divers champs d'activité. L'action prend forme à l'aide de ces ponts et quand l'information les emprunte, elle contribue grandement à orienter et soutenir l'ensemble des actions. Et inversement, ce sont ces mêmes actions qui donnent à l'information un sens, parce qu'elle est perçue comme utile dans l'entreprise et dans un réseau. Porter rend compte de cette réalité lorsqu'il écrit que : 
la technologie de l'information n'affecte pas seulement la manière d'accomplir les différentes activités : la circulation des informations permet aussi d'exploiter plus largement les liens entre activités, à l'intérieur comme à l'extérieur de l'entreprise. La technologie crée de nouveaux liens grâce auxquels les entreprises peuvent coordonner leurs actions plus étroitement avec celles de leurs acheteurs et fournisseurs (Porter 1999: 91).

Malgré les avantages qu'on lui reconnaît, le réseau et l'information qui y circule peuvent créer des situations embarrassantes, car la concurrence entre entreprises de même nature y subsiste et peut engendrer des attitudes et des comportements préjudiciables à la qualité des relations sociales. Petit, qui a recours à la notion d'encastrement social de l'information, n'ignore pas cette difficulté : «dire de l'information qu'il s'agî d'une relation encastrée consiste aussi à dire qu'elle s'insère dans un faisceau de relations sociales entre les individus et le monde dans lequel ils évoluent» (Petit 1998 : 71).

Le réseau demeure néanmoins le lieu de rencontre le plus approprié et le plus favorable à la résolution des divergences et à l'union des forces plutôt que l'action isolée. Observer l'action dans son cadre social, comme Petit le suggère, révèle l'importance des interactions suscitées par une information qui se propage. Or à sa façon, le réseau d'entreprises constitue un milieu social, et l'information qui y circule devient un facteur favorable à la ratification par le groupe de stratégies et de pratiques élaborées en commun. Il y a donc un intérêt certain à ce que s'effectue un couplage du flux de l'information stratégique avec le développement des réseaux d'entreprises.

L'information soude les structures internes de ces réseaux, agrandit les champs de coopération et de coordination entre les acteurs et fait voir des opportunités d'affaires parfois insoupçonnées. Souvent en effet, dans les interactions au sein d'un réseau, se trouvera le germe d'une innovation. Parlant des réseaux innovateurs, Ratti remarque que :

cette forme organisationnelle permet, soit des localisations d'entreprises plus stables, soit des localisations de nouvelles entreprises dans des régions excentriques, voire périphériques, par rapport à la théorie traditionnelle de localisation en termes de distance et de coûts de transport (...); Le réseau devient pour la firme un élément constitutif vital : il est en effet "le contexte approprié qui permet la combinaison créatrice des savoir-faire internes et externes de la firme" (...); Une importante question ouverte est celle de la territorialisation ou non des réseaux d'innovation : les relations en réseau - en principe réparties dans 
l'espace fonctionnel - ne trouveraient-elles pas dans la proximité un facteur essentiel de synergie ?(Ratti 1992 : 57).

Singh a observé que la diversité et la complexité des technologies que propose le marché sont l'une des raisons qui portent les entreprises à s'impliquer dans des réseaux et à coopérer. Il a montré que la pression d'une conjoncture quí contraint pratiquement au changement technologique peut provoquer l'échec des initiatives entrepreneuriales; que le recours à des alliances ou l'affiliation à des réseaux constituent les meilleurs moyens de franchir avec succès ces écueils. 11 constate toutefois que les effets varient d'un secteur d'activité et d'un territoire à l'autre (Singh $1997: 359$ ).

De même, Singh démontre empiriquement que le recours des entreprises à des alliances et leur affiliation à des réseaux n'assurent pas toujours la réussite; cela dépend largement des conditions de chaque entreprise et de l'environnement local et global qui encadre l'activité économique (Singh $1997: 360$ ). Selon l'auteur, chaque entreprise doit évaluer sa propre situation, considérer les stratégies qui lui conviennent le mieux, tout en tenant compte de ses propres besoins de développement et de sa capacité à profiter de l'information et de l'aide disponibles (Singh 1997:360). Il confirme que plus la technologie commercialisée par une entreprise est complexe, plus elle augmente les effets positifs des alliances inter-entreprises (Singh $1997: 346$ ).

Difficilement mesurable, l'influence d'un réseau vigoureux est néanmoins considérable. Dans un contexte où une information à jour est vitale pour la survie d'une organisation, l'entrepreneur peut souvent trouver auprès de pairs participant au réseau des renseignements qui, parfois, ne seraient accessibles que bien plus tard par les canaux officiels. Il peut dès lors prévoir: raffiner sa gestion, acquérir une nouvelle technologie, adapter sa structure organisationnelle de façon à tirer avantage d'un cadre commun, tout en renforçant l'efficacité des stratégies collectives. Ce faisant, il se prémunit contre les aléas inhérents au monde des affaires.

Toutefois le réseau, bien que fournissant un cadre commun et un encouragement à la concertation, ne vise pas à supprimer toute forme de concurrence ou de confrontation. La concurrence alimente en effet une saine émulation, stimule la vigilance et, malgré 
l'incertitude qui l'accompagne, elle est source de progrès. Le réseau peut sûrement contribuer à renforcer la confiance et atténuer l'incertitude décrite par Storper et al. :

placés en situation d'avoir à coordonner leurs actions de travail, d'offre et de demande (sinon le produit ne pourrait arriver à réalité), les acteurs économiques doivent être considérés comme s'affrontant à une incertitude radicale : sur le futur, sur l'action de l'autre, sur ses projets et ses attentes, sur les usages des objets présents (Storper et Salais 1993 : 13).

Même heureux en affaires, l'acteur économique est rarement à l'abri d'une vague incertitude, qui ne se réduit pas aux contraintes des règles du marché ou à des questions financières. La mondialisation de l'économie, les questions environnementales, la conjoncture socioculturelle, la dure concurrence des pays émergents qui invite à délocaliser l'entreprise, sont le reflet d'un contexte contemporain mouvant qui donne à l'incertitude des dimensions nouvelles et indistinctes.

Toutefois, la rapide obsolescence des techniques est sans doute le sujet qui retient le plus souvent l'attention. On peut en effet appréhender «l'incertitude technologique à travers trois problèmes emboités, de nature informationnelle et décisionnelle (...): quelles sont les technologies disponibles? Quelle est la technologie la plus appropriée aux besoins de la firme ? Comment utiliser au mieux la technologie retenue ?» (Charbit et Zimmermann 1997 : 10). La réponse à ces questions n'est pas simple. L'information en est évidement un élément essentiel et la participation à un réseau peut en être un autre.

\subsubsection{Réseaux et communication}

L'affiliation à des réseaux d'entreprises apporte souvent des réponses concrètes à ce genre de questions soulevées par l'incertitude. Elle fait voir aux acteurs l'intérêt d'améliorer leurs organisations puis à en faire profiter leur milieu. L'affiliation à un réseau, qui conditionne et est conditionnée par la circulation de l'information, incite l'acteur à se recentrer sur ses compétences de base, sur ce qui le différencie de ses concurrents. Le réseau atténue, pour l'entreprise, les répercussions défavorables des stratégies de ses concurrents, tout en favorisant lamélioration de sa performance. Au sein du réseau, linformation qui circule grâce à l'interaction entre participants devient un produit social, une «émanation directe du 
comportement matériel» (Marx 1966:50). Cette perception matérialiste de l'information lui permet de se réfracter sous forme d'actions de coordination, de concurrence ou de compétitivité : «la nécessité d'un agir coordonné produit dans la société une demande déterminée de communication, qui doit être satisfaite si une coordination effective des actions doit être possible afin de satisfaire les besoins» (Habermas $1987: 284$ ).

Dans un environnement changeant, les besoins ressentis de communication amènent les entreprises à coopérer entre elles et à coordonner leurs actions communicationnelles, ce qui se fait mieux à travers un réseau. Bien menée, cette collaboration agit comme le noyau d'un processus de communication de connaissances, décomposables en "processus de production" et en "processus d'acquisition" (Petit 1999: 28). Si un rapport causal apparait entre, d'une part les changement technologiques et autres, et d'autre part la nécessité pour l'acteur de s'ajuster à l'économie de l'information, c'est grâce aux actions communicationnelles, qui participent activement à l'évolution de logiques marchandes et non marchandes en harmonie avec les exigences d'un cadre commun.

On parle d'actions communicationnelles «lorsque les plans d'action des acteurs participants ne sont pas coordonnés par des calculs de succès égocentriques, mais par des actes d'intercompréhension» (Habermas 1987 : 295). Au cœur des actions communicationnelles, on trouve l'information, qu'il y a lieu de considérer comme un élément structurant des réseaux d'entreprises. L'information n'est pas seulement une marchandise, elle n'est pas un simple facteur de compétitivité et de concurrence, elle n'a pas seulement un «caractère stratégique» (Petit 1998 : 33) ; elle est tout cela. Elle est une vraie relation, dont la gestion englobe ses «politiques d'accompagnement» (Petit $1998: 33$ ).

Les réseaux d'entreprises sont donc des champs où prennent forme les actions communicationnelles qui donnent à l'information sa vraie valeur. Or, croire que tout le monde peut acquérir cette information n'importe où et partout est une illusion. Cette acquisition dépend des compétences existant chez ses acquéreurs, et beaucoup de la force des liens qui se nouent au sein des réseaux d'entreprises: "pour trouver l'information adéquate, il faut disposer du savoir préalable qui permette de poser les bonnes questions» (Sfez $1999: 22$ ); ce savoir peut souvent être acquis par les échanges face-à-face qui ont lieu entre acteurs au sein d'un réseau. 
L'absence de ce savoir préalable peut desservir les acteurs, à la recherche d'information pour la poursuite de leurs objectifs de développement. Et pour aller chercher les bons renseignements, ils doivent avoir une idée assez précise de ce qui peut être utile dans une situation problématique. Les membres d'un réseau d'entreprises sont alors à même de comprendre les particularités d'un contexte où le partage d'information concourt à la combinaison, dans une seule action, de «la technologie de la production du savoir, du traitement de l'information et de la communication des symboles» (Castells 1998 : 38).

\subsection{Les réseaux d'entreprises et l'apprentissage}

Le réseau d'entreprises offre un soutien qui peut être déterminant, aux entreprises qui veulent apprendre à résoudre collectivement les problèmes rencontrés individuellement. La circulation des connaissances que permet le réseau peut être un tremplin pour l'innovation

\subsubsection{Réseaux d'entreprises et circulation des connaissances}

Entre les membres d'un réseau d'entreprises, des connaissances circulent, très utiles pour identifier les remèdes à la morosité de l'incertitude ou d'une situation qui risque de gêner le développement de l'entreprise. Ces connaissances apparaissent «comme fortement subjectives, intrinsèques aux individus, complexes et étendues» (Mayère 1997: 134); elles émanent de ces entrepreneurs qui ont su, chacun dans son milieu, donner une couleur originale au développement économique :

the local nature of entrepreneurship bolsters the finding of the local specificity of economic development, "entrepreneurial vitality is very much a local phenomenon (...) prosperity and economic growth of regions and localities are strongly associated with the strength and vitality of the small firm sector in the region or locality (Malecki 1991: 323).

Influencé par les transformations qui amènent l'informatique dans les domaines de l'information et de la communication, l'espace entre les dimensions locale et globale tend à s'estomper. Bien que plusieurs facteurs économiques, socioculturels et géographiques façonnent cette influence, le défi premier qui s'impose à l'acteur résulte du «caractère très instable des systèmes actuels (qui) nous oblige à adapter régulièrement notre compétence pour 
mieux gérer les évolutions du "système entreprise" et remet en cause en permanence l'expérience đu passé») (Bouchy 1994 :29).

Affronter linstabilité, lincertitude, la concurrence, a toujours été pour l'acteur économique la règle du jeu. Toutefois, l'avènement des TIC et la globalisation du marché tendent à déplacer le niveau de la concurrence, et c'est la nature de la concurrence et les succès de l'acteur à s'y mesurer qui décident, en définitive, de la dynamique locale et régionale du développement. La compétition devient de plus en plus nationale et mondiale, et force l'acteur à s'ajuster. Porter souligne l'importance de cette évolution:

alors que la concurrence est en train de devenir de plus en plus nationale et mondiale, les sources essentielles d'avantage concurrentiel demeurent souvent locales. Elles résident dans des masses critiques de compétences hautement spécialisées et liées entre elles, de technologies appliquées, d'entreprises, de foumisseurs et d'institutions bien localisées (Porter 1999 : 325).

Quelles que soient les origines de la concurrence, celle-ci n'est pas la source unique des contraintes que subissent les acteurs. C'est pourquoi le milieu des affaires semble toujours plus ou moins en effervescence, ayant à composer avec une conjoncture mouvante, parfois la précarité, les revendications syndicales, la réglementation, etc. La possibilité d'affrontement est donc liée à une multitude de situations ; et chacune se démarque par la présence d'un certain absolu qui facilite son intégration dans les schémas rationnels (Petit 1998:33) C'est pourquoi l'issue de l'incertitude est souvent influencée non seulement par la nature de la situation en question et par les intérêts qu'elle met en jeu, mais surtout par les choix que doit faire l'acteur pour s'en sortir. Robert Salais écrit :

c'est l'épreuve de réalité qui dénoue lincertitude et arrête pour chacun la quête d'informations sur ce qu'il fait et sur ce que font les autres (...) ainsi, si nous sommes capables de dégager les modalités possibles de cette épreuve, ne pouvons-nous, par une sorte de remontée du raisonnement (backward induction), délimiter les formes correspondantes de traitement de l'incertitude dans le cours, "antérieur", de l'action de travail et, ainsi, les conventions de travail mises en cuvre (Salais 1994 : 379). 
L'évolution continue de la technologie est sans doute une source d'instabilité et d'incertitude; par contre la généralisation de l'informatique a entraîné un recours plus fréquent à l'information spécifique au champ d'action de l'entreprise.

Malecki et Verdhoen (1993) ont analysé le rôle des réseaux d'entreprises dans un contexte dominé par l'information et où la compétition entre les petites entreprises est la règle. Pour les fins de leur étude, les auteurs ont utilisé des données sur la taille des entreprises, les rapports entre celles-ci, les marchés, les flux de l'information, de même que sur les activités de recherche et développement et sur l'innovation. Leur recherche indique qu'en général, les entreprises qui diversifient leurs sources d'information sur les marchés, sur les concurrents et sur les nouvelles technologies offrent davantage de nouveaux produits et services. De même, l'analyse des sources d'information de ces entreprises montre que toutes les entreprises comptent sur les expositions commerciales et sur les conférences régulièrement organisées comme principales sources d'information. Enfin, l'analyse a démontré que plus une entreprise s'affilie à un réseau important, plus elle tire profit de l'information et de son économie.

Que l'utilisateur de l'information soit désormais vu presque comme un rouage normal d'un système révèle un déplacement des axes de l'action économique et socioculturelle. Il y a là en effet une situation nouvelle qui développe, autant qu'elle résulte, chez les acteurs d'un réseau d'entreprises, des compétences qui les font se rapprocher davantage, adopter des stratégies communes de développement, en s'entendant sur des priorités et surtout en se préparant collectivement à mieux affronter les conséquences de l'évolution des systèmes en place.

Quand un acteur s'implique dans un réseau, il en espère ce supplément que donne aux interactions entre pairs la circulation, avec un minimum de contrainte, d'information et de connaissances tacites. Pour tirer profit des réseaux d'entreprises, les acteurs doivent savoir utiliser l'information stratégique disponible; toutefois cela en effet ne se traduit en développement que si certaines conditions préalables sont réunies. L'une de ces conditions est la mise en place d'un système de gestion souple, qui permette une allocation optimale des ressources dont dispose l'entreprise. Puis misant sur la formation, du haut en bas de l'entreprise, les dirigeants doivent être capables de surmonter les problèmes de gestion pouvant découler du changement dans leur milieu. Cette pression les amène à reconnaître l'utilité de se joindre à un réseau d'entreprises, qui se révèle alors comme une méga- 
organisation apte à les avantager en créant des ponts plus faciles à traverser à l'intérieur d'un même marché, des ponts qui relient aussi au dynamisme de différentes entreprises, et facilitent la tâche. Les avantages sont évidents :

les réseaux permettent des transferts d'information de marché (entre acheteurs et offreurs, entre fournisseurs et utilisateurs, etc.) ou hors marché, comme le développement d'idées nouvelles par l'examen de différentes possibilités ou par des opportunités reconnues par le réseau. Ils tendent ainsi à favoriser la saisie de toutes sortes d'occasions d'affaires. Lorsque l'information échangée est particulièrement abondante, elle permet d'accélérer l'innovation dans le milieu (Julien $1996: 223$ ).

Un acteur peut apprendre davantage de l'expérience de ses pairs s'il dispose d'une information pertinente sur les forces et les faiblesses de son milieu et aussi sur les siennes. C'est souvent dans le réseau, auprès de ses pairs, que le dirigeant va trouver le soutien nécessaire pour oser, pour innover. Cela vaut manifestement pour l'acteur individuel mais aussi, au détour des événements qui affectent l'un ou l'autre, pour l'ensemble des membres du réseau. La raison en est que, en définitive, c'est l'information qui circule dans le réseau qui aide à souder des structures entre participants, consolider les acquis, mais surtout à stimuler l'évolution et le développement. Fort de solides expériences locales, le réseau permet de franchir les cadres territoriaux, d'élargir l'horizon d'un utilisateur frileux et, par la coordination des efforts de poursuivre, en plus d'objectifs économiques, des buts à caractère social. On remarque en effet que

la poursuite d'objectifs économiques s'accompagne normalement de celle d'autres objectifs de nature non économique, tels que la sociabilité, l'approbation, le statut social et le pouvoir. L'action économique (comme toute action) est socialement située et ne peut être expliquée par de simples motifs individuels; elle est encastrée dans le réseau des relations personnelles, plus qu'elle n'émane d'acteurs atomisés (Granovette 1994 : 81).

L'affiliation à un réseau d'entreprises est souvent stimulée par la quête d'un ensemble de valeurs, de normes, mais aussi d'objectifs et de stratégies. Au départ, cette affiliation se révèle comme une recherche de force supplémentaire, par laquelle le dirigeant d'entreprise espère déjouer une concurrence reposant davantage sur les subtilités des règles du marché, que sur 
un produit vraiment supérieur. L'affiliation augmente sa capacité concurrentielle et permet la mise en place de conditions permettant d'affronter collectivement les situations d'incertitude.

La concurrence toutefois ne disparait pas pour autant. S'estompant d'un côté, elle peut surgir de l'autre. Et l'acteur avisé, même appuyé par un réseau, doit d'abord compter sur lui-même et savoir se doter des technologies les plus aptes à assurer son succès. À cet égard la technologie de l'information devrait sans doute venir au premier rang. Porter en résume les effets sur la concurrence :

la technologie de l'information modifie les règles de la concurrence de trois manières. D'abord, les progrès des technologies de l'information modifient les structures sectorielles. En second lieu, les technologies de l'information constituent un levier de plus en plus important, utilisable par les entreprises pour se constituer un avantage concurrentiel. Et quand une entreprise cherche à obtenir un avantage concurrentiel grâce aux technologies de l'information, cela finit souvent par affecter toute son industrie, car les concurrents imitent les innovations stratégiques du leader. Enfin, la révolution de l'information suscite la création d'entreprises totalement nouvelles. Pour comprendre l'effet des technologies de l'information sur une industrie donnée et pour mettre au point des réactions stratégiques efficaces, il faut tenir compte de ces trois effets (Porter 1999 : 94).

Bref, la concurrence fait partie de la vie de l'entreprise : savoir y résister ou disparaître. À chacune elle impose un parcours particulier, virtuellement déstabilisateur de sa structure organisationnelle. Alors l'affiliation à un réseau prend tout son sens; là peut s'établir une proximité de vues, une concertation des stratégies, où le dirigeant reçoit appui, profite de l'expérience de ses pairs et acquiert des connaissances utiles à son organisation. Ainsi est atteinte une proximité institutionnelle qui

exprime l'adhésion d'agents à un espace commun de représentation, de règles d'action. Cette proximité est étroitement liée à des interactions entre agents qui peuvent fonder l'émergence d'un territoire à partir d'un processus d'apprentissage collectif. Celui-ci peut impliquer une proximité géographique entre les agents, dans la mesure où celle-ci est une condition de la formation de relations durables, vecteur d'échanges non codifiables c'est-à-dire non transportables dans l'espace par des supports techniques. Elle s'inscrit dans les réseaux sociaux (Dupuy et Filippi 2000:727). 
Ce passage montre l'importance du réseautage comme ferment d'une proximité sociale et professionnelle débouchant naturellement sur l'apprentissage. Mais pour une organisation, l'apprentissage (considéré comme un processus) de ses cadres ou de ses employés n'a d'intérêt que s'il amène à une connaissance, un savoir-faire qui permettent d'abord de résister à la concurrence et, avec un peu de chance et beaucoup de travail, d'innover.

\subsubsection{L'apprentissage et l'innovation}

Plusieurs études ont déjà constaté une corrélation positive entre l'apprentissage et l'innovation. Par exemple, Stein (1997) a montré que l'innovation peut découler de la capacité d'une entreprise à améliorer la qualité de sa production ou à tirer avantage de son habileté à commercialiser sa production. Dans ces deux cas, l'entreprise renforce son expertise par le développement de son savoir-faire, soit, souvent, en favorisant l'apprentissage sur le tas, soit en incitant le personnel à profiter d'une formation sur mesure (Stein 1997). Or cette ligne de conduite exige prévoyance et vigilance; une entreprise qui encadre bien ses pratiques se dote généralement d'une politique convenable de formation et d'une stratégie claire d'apprentissage; d'où l'importance du 'learning-by-doing' (Stein 1997). De même, Sole et Edmondson (2002) ont montré que la diversité de la connaissance et la capacité d'apprentissage d'une entreprise dépendent largement de sa localisation, et donc du contexte général qui encadre ses activités. De plus, l'apprentissage dépend principalement de l'effort des individus et de leur aptitude à mieux s'intégrer aux communautés auxquelles ils appartiennent.

Certes, l'apprentissage se fait dans un terrain social. Mais, tout acteur affronte un contexte économique qui imprègne cet apprentissage et influe sur l'usage qui sera fait des connaissances acquises. Les changements possibles, pour un acteur impliqué dans un réseau, offrent plusieurs éventualités parmi lesquelles il doit choisir en fonction de la conjoncture : rapidement il devra adapter son outillage et son savoir-faire à la nouvelle réalité. Malecki voit deux façons d'y arriver:

the most elementary form of learning is learning by operating, a variant of learning by doing or by using. On the whole, the enhancements are rather small. The second form of learning is learning by changing: improving upon equipment and techniques subsequent to gaining experience with them (Malecki 1991: 147). 
L'apprentissage est donc souvent à l'image du parcours collectif, qui offre au dirigeant des éléments de réponse à une situation préoccupante. Il est «le produit de l'expérience accumulée au cours de la production et il ne peut se dérouler que lorsqu'on cherche à résoudre un problème» (Amable et al. $1997: 54$ ). Il faut donc concilier deux nécessités : affronter les situations d'incertitude occasionnée par l'économie d'information ; apporter des ajustements organisationnels qui tiennent compte du devenir à la fois de l'entreprise et de son milieu. L'apprentissage n'est donc pas le seul fait du dirigeant, mais d'une équipe.

Le cadre général des pratiques collectives, de même que le degré de compétitivité du système socio-économique en place, influent sur l'apprentissage. Tout dirigeant d'entreprise est en quelque sorte obligé d'inscrire sa démarche dans la logique de cette réalité. De là l'utilité des réseaux d'entreprises qui encouragent l'apprentissage dans tous ses états et dans toutes les directions. Cette utilité vient aussi du poids des réseaux comme partie intégrante d'un environnement spécifique: ils sont alors considérés comme un cadre offrant un espace fonctionnel à caractère discret (Ratti 1992 : 56). Les objectifs qui président à la création d'un réseau et ceux qui incitent une entreprise à s'y affilier sont naturellement convergents. Ratti mentionne les plus importants :

les objectifs de la stratégie d'organisation de réseaux (...) : Réaliser des économies d'échelle et des économies de "scope" (de variété) à travers la mise en commun d'expériences en matière de R\&D (c'est l'objectif le plus traditionnel et valable en particulier pour de grands complexes); Limiter les coûts de recherche, d'appréciation et de décodification concernant de multiples et complexes inputs, d'un savoir technique et scientifique, afin de pouvoir les transformer en savoir-faire; Gérer, d'une façon optimale, la trajectoire de développement d'une nouvelle technologie ou de composantes technologiques nouvelles (Ratti $1992: 56$ ).

On met donc en commun des compétences variées pour mieux apprendre collectivement et améliorer la compétitivité des entreprises participantes. Mais chez elle, la firme doit composer avec ses propres impératifs techniques. Les connaissances doivent passer dans la pratique quotidienne et l'apprentissage dans chaque poste de travail leur donner une utilité concrète :

firms must be able, once they have created or acquired tecknology, to nourish and sustain it in production. Especially for process technology, learning by using and by doing are essential components of the technological activities of firms. 
Firms have three ways of adding to their stock of knowledge : through learning by doing and using, through R\&D (or learning by studying), and through imitating others (diffusion between firms) (Malecki 1991: 154).

Selon les conditions existant dans une entreprise et le type de savoir-faire recherché, un mode d'apprentissage apparaîtra, mieux qu'un autre, capable de répondre au besoin. Pour une technologie de méthode (processus technologique), l'apprentissage en "utilisant" et en "faisant" est un élément essentiel de l'appropriation technologique. "Learning by using" correspond bien à la démarche d'appropriation des TIC : il n'y a qu'une seule façon d'arriver à les bien maîtriser, c'est en les utilisant. Naturellement la maîtrise d'une nouvelle méthode, d'un outil nouveau requiert un certain effort, parfois fastidieux. Dans le cas des TIC, cet effort, peut-être davantage que le coût en argent, pourrait freiner leur appropriation. Cette appropriation exige en effet, au-delà d'un apprentissage théorique minimal, une bonne réserve de patience, de temps d'essai - erreur qui peuvent paraître décourageants lorsqu'on méconnaît les avantages considérables de cette appropriation.

\section{Conclusion}

On a vu au premier chapitre que des rapports étroits existent entre le changement technologique et l'innovation. $\grave{A}$ notre époque, c'est l'informatique qui semble constituer un moteur principal du changement technologique et en explique les rapports à l'innovation. L'informatique, et particulièrement les TIC, ont une influence considérable sur les pratiques des acteurs individuels et collectifs. Les changements technologiques semblent modifier graduellement les normes, voire les valeurs dans les pratiques entrepreneuriales, les adaptent aux exigences conjoncturelles et structurelles du moment et suggèrent les conduites propices à la réussite en affaires.

Ces idées sont un condensé des apports de plusieurs chercheurs qui se sont intéressés aux façons originales dont les acteurs, tant de la scène économique que sociale, réagissent à l'arrivée d'une technologie de pointe qu'ils jugent opportun d'apprivoiser. Les conséquences qui en découlent, reflets de dynamiques nouvelles enracinées dans le terreau culturel et social, tendent à consolider les atouts des territoires et les avantages des acteurs qui savent en profiter. A cet égard l'appropriation des TIC s'avère important pour les pratiques 
entrepreneuriales et il devient nécessaire, pour les pouvoirs publics, de simpliquer activement dans un secteur aussi stratégique.

Dans ce deuxième chapitre, nous avons tenté de comprendre comment les réseaux d'entreprises influent sur les pratiques entrepreneuriales, par la circulation de l'information, l'apprentissage et l'utilisation des TIC. Plutôt qu'une sorte de fuite en avant que choisiraient les acteurs confrontés à l'incertitude, l'affiliation à un réseau d'entreprises nous est apparue comme une stratégie d'affaires en vue d'apprendre, de se concerter et d'affronter collectivement les aléas du marché. L'affiliation d'une entreprise à un réseau est souvent dictée par la conjoncture et a comme toile de fond la circulation de linformation et son utilisation dans l'entreprise et dans la collectivité.

La compréhension des rapports et de l'influence réciproque entre TIC, réseaux et information demeure imparfaite, pour plusieurs raisons, malgré l'intérêt que divers chercheurs ont accordé à ces questions. Parmi les principaux facteurs il y a d'abord l'incertitude des acteurs de développement, générée par l'instabilité de la conjoncture internationale et ses inévitables répercussions au niveau de l'entreprise. L'arrivée massive des TIC, la mondialisation qu'elles favorisent et la nouvelle économie de l'information qu'elles entrainent, sont autant de motifs de remises en question souvent pénibles. Par ailleurs le chercheur, comme l'acteur, doit composer avec la diversité et la multiplicité des réseaux, et aussi l'ambiguité de l'ordre informationnel, dont les messages ne sont pas nécessairement concordants.

Le développement des réseaux d'entreprises, qui s'avèrent néanmoins de plus en plus nécessaires, invite à placer les pratiques entrepreneuriales dans un contexte largement influencé par l'usage des TIC et par les processus liés à l'information et à son économie. La décision d'un acteur de s'affilier à un réseau se fonde désormais moins sur un champ d'intérêt précis ou un cadre spatial déterminé, mais tend à s'inscrire dans une vision entrepreneuriale élargie par l'usage généralisé des TIC. Enrichis des apports d'une technologie de plus en plus sophistiquée, les acteurs participent à la création de réseaux dont ils déterminent les formes en redéfinissant sans cesse l'usage optimal de l'information disponible. On assure ainsi une certaine concordance entre les dimensions technologiques, économiques et sociales de l'information, et se développe en même temps un ensemble de règles sur lesquelles se fonde 
l'action. L'aspect économique se trouve donc immédiatement traduisible en comportements de coordination, et ultimement sociaux.

Pour l'acteur à la recherche de cadres appropriés pour un apprentissage de qualité, le réseau d'entreprises offre des possibilités intéressantes. Opposé à la routine, lorsqu'il devient un rouage normal du fonctionnement d'une entreprise, l'apprentissage est un puissant facteur de changement et d'efficience, parce qu'il n'est jamais un acquis définitif mais exige la mise à jour constante des connaissances. Il repose donc sur des connaissances préalables, un certain savoir-faire et une attitude d'ouverture à la nouveauté.

Par ailleurs la position de l'entreprise dans son environnement lui impose de tenir compte de l'évolution de la conjoncture, tant technologique que sociale. D'une part la société attend d'elle qu'elle agisse en " bon citoyen corporatif", en même temps qu'elle est de plus en plus confrontée à des exigences qui lui sont propres: de nature environnementale, réglementaire ou autre, qui requièrent un haut degré de compétence, parfois de diplomatie de la part du dirigeant, et d'harmonie dans l'organisation. D'autre part l'évolution technologique, la mobilité des capitaux, la volatilité de la conjoncture, la concurrence, soumettent l'entreprise à des pressions considérables pour survivre et si possible, crôtre.

À certains égards, on peut être tenté de dire que les TIC font partie en même temps du problème et de la solution. Au départ, l'instauration de cette nouvelle technologie peut compliquer le fonctionnement d'une firme, car pour s'y familiariser une période de rodage est nécessaire. Mais dès qu'est réalisée une appropriation convenable, la gestion et le fonctionnement y gagnent énormément en efficacité et en précision. Le réseau d'entreprises constitue, pour les participants, une source d'appui supplémentaire, un lieu d'information, où peuvent s'élaborer des stratégies communes, où, en somme, apprendre collectivement à se tirer d'affaire individuellement. 


\section{CHAPITRE 3}

\section{LES EFFETS DE LA TAILLE DES FIRMES}

Ce chapitre présente un aperçu des contributions les plus significatives que des chercheurs ont fournies à partir du deuxième tiers du XXe siècle, relativement au rapport existant entre la taille d'une entreprise et l'innovation. Ces chercheurs eurent un devancier important, Schumpeter, dont il y a lieu d'abord de rappeler les principales idées sur cette question, notamment sa perception des processus de "destruction créatrice" et leurs rapports avec l'innovation. Vient ensuite un examen des constats de plusieurs investigateurs, qui se sont intéressés aux effets de la taille d'une entreprise sur l'innovation qu'elle génère. Certains ont conclu que plus grande est l'entreprise, plus elle est innovante. D'autres, qu'il y a peu de relation entre la taille d'une firme et l'innovation, alors que d'autres encore voient une relation négative. Il semble que, outre la taille, d'autres facteurs, notamment conjoncturels, influent sur l'innovation. 


\subsection{Aux origines du débat}

Pour l'avancement de la connaissance des dynamiques économiques et sociales de développement, les travaux de Schumpeter constituent une œuvre charnière. L'originalité de sa contribution tient principalement à un examen critique du rôle des entreprises dans les dynamiques de développement. Afin de comprendre le fonctionnement de ces dynamiques, il a cherché à discerner la nature de la relation pouvant exister entre la taille d'une firme et l'innovation. Ses recherches le convainquirent de l'existence d'une telle relation, qui peut prendre des formes originales et exprimer la vitalité d'une économie.

\subsubsection{La pensée de Schumpeter}

Des écrits novateurs de Schumpeter se dégage une relation, à première vue paradoxale, entre la taille d'une entreprise et l'innovation. Son ouvrage de 1935, "Théorie de l'évolution économique : recherches sur le profit, le crédit, l'intérêt et le cycle de la conjoncture» présente ses premières idées et avance la thèse que les petites entreprises sont, davantage que les grandes, la source du progrès technologique. Par la suite, dans «Capitalisme, Socialisme et Démocratie», il développe l'idée que les grandes entreprises sont plus enclines à innover que les petites. On ne saurait toutefois inférer de cette apparente contradiction que la pensée de Schumpeter est incohérente. Des circonstances différentes expliquent des conclusions contrastées, comme on verra plus loin. En réalité, sa pensée suit essentiellement un cheminement intellectuel structuré et conséquent.

Deux synthèses importantes jalonnent le parcours de Schumpeter: "Théorie de l'évolution économique», publié en 1935 et, en 1939, «Business Cycles, a Theorical, Historical and Statistical Analysis of the Capitalist Process».

Dans ces synthèses, Schumpeter met en relief le rôle de l'entrepreneur et insiste pour distinguer entre l'innovation, qu'il considère comme le moteur de la croissance économique, et l'entrepreneur, souvent présenté "comme le phénomène fondamental», qui marque les dynamiques économiques existantes (Schumpeter 1935:330). Les entrepreneurs sont des «agents économiques dont la fonction est d'exécuter de nouvelles combinaisons et qui en sont l'élément actif» (Schumpeter 1935:330). L'acteur n'est «entrepreneur que s'il exécute de 
nouvelles combinaisons - aussi perd-il ce caractère sil continue ensuite d'exploiter selon un circuit l'entreprise créée» (Schumpeter 1935:336).

Dans les écrits de Schumpeter, les liens entre les nouvelles combinaisons et le développement d'une entreprise sont mis en relief à plusieurs reprises. L'auteur estime que la multiplication des activités innovantes renforce la place de l'entreprise dans son environnement économique, car il suffit généralement que les entrepreneurs décident d'affecter les ressources nécessaires à l'application des nouvelles méthodes de production, pour obtenir une productivité avancée (Schumpeter 1939: 85).

Schumpeter s'attache à préciser le rôle que jouent les entreprises dans leur contexte économique. Il montre qu'un entrepreneur prend des décisions de nature à créer les conditions favorables aux nouvelles combinaisons de facteurs de production. Autrement dit, un entrepreneur innove en prenant en considération les différents mécanismes conjoncturels, de façon à y adapter convenablement son entreprise. C'est bien dans ce sens que la pensée de Schumpeter donne aux petites entreprises un rôle central dans les dynamiques économiques. L'entrepreneur, qui doit être bien engagé dans son milieu, doit en quelque sorte innover s'il veut continuer d'exister, et donc être plus productif et plus concurrentiel. Schumpeter estime que l'importance de l'engagement d'un entrepreneur dans son milieu lui permet de bénéficier du genre de soutien dont il peut avoir besoin.

Vues dans un autre contexte, les grandes entreprises se révèlent à Schumpeter plus conservatrices quant à l'innovation, mais plus capables de s'en approprier les bénéfices :

«dans l'ère des entreprises géantes, le maintien de la valeur des investissements antérieurs - conservation du capital - devient le principal objectif de l'activité des entrepreneurs et tend, selon toute apparence, à bloquer toutes les améliorations susceptibles de comprimer les prix de revient» (Schumpeter $1942: 138$ ).

Schumpeter attire ainsi l'attention sur le fait que les grandes entreprises voient un avantage à développer leur capacité de s'approprier les bénéfices de l'innovation, mais qu'elles n'accordent qu'une importance modérée à la production de cette même innovation. Il souligne que les grandes entreprises sont susceptibles d'adopter de nouvelles méthodes de production si celles-ci occasionnent des coûts moindres (Schumpeter 1942 : 139). Ce choix lui paraît 
totalement justifié, car les entreprises de cette catégorie possèdent généralement les moyens importants et nécessaires pour la mise en œuvre de toute recherche pertinente : «dès qu'une grande entreprise se sent en mesure d'en couvrir les frais, elle s'empresse (aux Etats-Unis) d'installer un service de recherche dont chaque agent sait que son gagne-pain dépend du succès avec lequel il mettra au point des perfectionnements inédits» (Schumpeter 1942 : 139).

On le voit, Schumpeter associe l'innovation tantôt aux pratiques des petites entreprises, et tantôt aux initiatives par lesquelles les entreprises géantes agissent sur leur milieu. Cette perception entraîne l'abandon de l'hypothèse qui lie l'innovation à la taille d'une entreprise, quelle soit petite ou géante. Pour la capacité innovatrice d'une entreprise, ce qui compte en définitive, c'est sa nature, le contexte où elle évolue et les objectifs qu'elle poursuit. Dans la pensée de Schumpeter, l'innovation ne peut plus être uniquement affaire de taille, mais plutôt d'aptitude à performer et à consolider les liens forts qu'une firme entretient avec son milieu.

Par ailleurs, Schumpeter reconnaît qu'un facteur d'ordre géographique ou régional peut être le moteur des forces de changement: ce facteur «révolutionne sans cesse la structure économique de l'intérieur, détruisant sans cesse l'ancienne structure, en créant sans cesse une nouvelle» (Schumpeter 1942: 83). Les donnes géographiques concourent ainsi aux dynamiques économiques que subissent les divers processus de destruction créatrice. Ces processus conditionnent le fonctionnement des activités économiques, qui connaissent d'abord une phase de crise, où les grandes orientations conjoncturelles sont remises en question; vient ensuite une autre phase où les petites innovations prennent de l'ampleur, donnant à l'activité économique un nouvel essor. Cette succession résume le déroulement des processus de destruction créatrice et, en même temps, résume un développement du genre prospérité - récession - dépression- reprise. Un processus de destruction créatrice fait référence à la coexistence dans une seule action de deux tendances simultanées : l'une tend à détruire un cadre existant, en même temps qu'une autre logique s'affirme comme porteuse d'un avenir amélioré, qui cherche à prendre place.

Dans ses ouvrages, Schumpeter développe ce qu'il est convenu d'appeler l'approche économique de l'innovation. Selon cette approche, l'innovation stimule le développement économique, car elle est souvent à l'origine d'un nombre important de processus de destruction créative ; processus qui, eux-mêmes, provoquent souvent le renouvellement des 
structures en place. De même, les implications de l'innovation en termes de cycles d'affaires peuvent être appréhendées à partir de cette notion de "destruction créatrice" et de son incidence sur les dynamiques des systèmes en place. Les divers processus liés à ce principe de destruction créative sont souvent responsables de la disparition de produits et de structures caducs. La croissance d'une économie résulterait donc des dynamiques conséquentes, épaulées en grande partie par les efforts des grandes entreprises. De ce point de vue ces dynamiques expliquent comment l'évolution économique impose le passage à une situation où l'innovation règne et devient alors la plate-forme des changements spontanés et qualitatifs des activités économiques.

Schumpeter a perçu une relation intéressante entre la taille de l'entreprise et l'innovation. Selon lui, et comme l'a constaté Ratti, l'innovation est liée à une variable exogène : le progrès scientifique qui, lorsqu'il intègre l'activité productive «peut être à même, surtout auprès des grandes entreprises, de générer des feed-back permettant d'autres découvertes scientifiques et technologiques» (Ratti 1992 : 26). C'est que les entreprises fonctionnent dans un contexte caractérisé souvent par "des routines et par des innovations", que l'on peut considérer à l'origine de toute évolution économique.

\subsubsection{Interprétation et utilité des perspectives de Schumpeter}

Les idées de Schumpeter concernant les circuits économiques et leur évolution ont créé une certaine confusion, qui a conduit à des interprétations variées. Plusieurs auteurs ont vu les risques de cette confusion et ont cherché à y obvier en mettant surtout l'accent sur les points forts de la pensée Schumpeterienne. Par exemple, Ratti rend compte de la compétition entre les entrepreneurs et souligne l'importance des raisons qui poussent un entrepreneur à agir dans des conditions de croissance imparfaites. Il écrit :

«la plus importante de ces raisons étant l'existence d'un 'time lag' (d'un décalage temporel): 1. entre le moment où se vérifie la nécessité d'une augmentation de capital et le moment où il est à disposition de l'entrepreneur; 2. ou dû aux frictions existantes au moment de substituer les facteurs de production (capital et travail, rigidité des salaires) ; 3. ou, enfin, parce que l'innovation ne serait pas distribuée d'une façon homogène entre les secteurs économiques. Ces différents rapports de forces entre secteurs et l'existence de monopoles représenteraient d'autres sources de déséquilibre» (Ratti 1992:27). 
En fait, se basant sur la perspective de Schumpeter, Ratti constate que lopportunité technologique et l'opportunité du marché constituent un soutien extraordinaire à l'innovation. Cette constatation peut être déduite de la figure 1, conçue par Ratti pour illustrer à quel point le progrès scientifique importe pour l'innovation. Dans un premier temps, ce progrès est considéré comme une variable exogène, qui devient, par la suite, une composante principale des activités entrepreneuriales. Comme le montre cette figure, ce sont les initiatives des grandes entreprises qui en profitent en premier lieu, et ce sont elles qui produisent des rétroactions, nécessaires, en définitive, à l'évolution de la science et des technologies.

Figure 1: Le modèle de Schumpeter

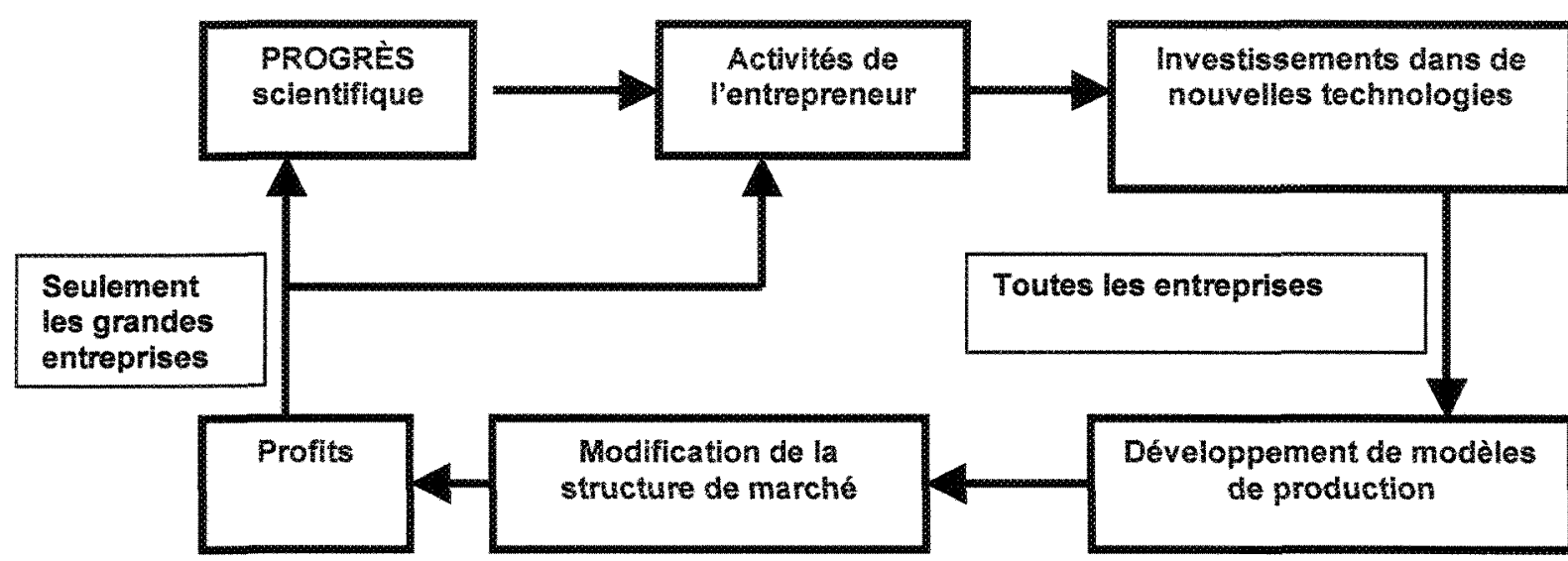

Source : Ratti, R., (1992), Innovation technologique et développement régional, Lyon, Presse Universitaire de Lyon, p. 27.

Comme Ratti l'a résumé dans la figure 1, la pensée de Schumpeter fait voir que la croissance «se fait en cycles longs, elle est déséquilibrée et présente une diffusion non uniforme» (Ratti 1992 : 27). Cette idée a poussé Florida à préciser que la croissance économique, tout comme tout processus de destruction créatrice, «s'étend aussi aux phénomènes régionaux, dans la mesure où les nouvelles technologies et les nouvelles formes d'organisation de la production ne se contentent pas de s'appliquer aux nouvelles régions, mais informent et modèlent la reconstitution ef la revitalisation des régions existantes》 (Florida $2000: 360-361$ ). 
Ayant observé l'association pouvant exister entre l'envergure d'une entreprise et sa capacité innovatrice, Schumpeter offre des conclusions contrastées. Il constate, d'une part, une relation étroite; d'autre part, que l'innovation n'est pas un phénomène propre aux grandes entreprises, même si elles sont généralement les premières à pouvoir profiter des progrès scientifiques, mais s'étend aussi aux petites entreprises qui valorisent l'initiative. En fait, la contradiction n'est qu'apparente. Ayant fait ses observations dans des contextes différents, l'auteur avait vu que cette relation est constamment conditionnée par des facteurs conjoncturels.

On trouve donc chez Schumpeter deux visions différentes, qu'une abondante littérature

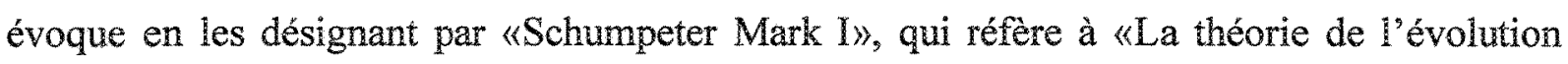
économique» et par «Schumpeter Mark II» qui renvoie au «Capitalisme, Socialisme et Démocratie». Cet apparent dédoublement de perception que l'on constate chez Schumpeter s'explique par deux contextes fort différents, qu'il a analysés avec rigueur. Parlant de «Théorie de l'évolution économique», Malerba et Orsenigo écrivent: "In this work, Schumpeter examined the typical European industrial structure of the late nineteenth century, characterized by many small firms» (Malerba et Orsenigo 1995: 47). L'autre contexte réfère à la situation nord-américaine.

Schumpeter a fait des émules qui se sont employés à raffiner les connaissances déjà acquises. Les sections qui suivent présentent un aperçu de l'éventail d'opinions, souvent fort divergentes, qui se sont exprimées dans la littérature contemporaine, relativement à l'influence de la taille d'une firme sur l'innovation.

\subsection{L'innovation s'accroît avec la taille de la firme}

Plusieurs études, datant des années cinquante et soixante, ont appuyé l'hypothèse voulant que l'innovation dans une entreprise s'accroît avec sa taille. Villard fut l'un des principaux auteurs à défendre avec détermination cette opinion. Son approche s'inspire des changements qui secouent la société américaine vers la fin des années 1950 et le début des années 1960. Durant cette période, la grande industrie des États-Unis procède à des transformations devant lui permettre de confirmer sa supériorité face à une économie soviétique stimulée par les enjeux de la guerre froide. Les grandes entreprises devaient assumer l'effort industriel du pays, laissant au gouvernement central de s'occuper des affaires stratégiques. Villard estimait en 
effet que les petites firmes ne peuvent guère s'impliquer dans l'innovation sans l'appui de l'État: «I do not see how small firms can, in practice, finance big laboratories except cooperatively or with government aid» (Villard 1958: 495-496).

$\mathrm{Si}$, aux yeux de Villard, la grande entreprise a un rôle prépondérant par rapport aux petites, c'est qu'elle répond à une forte demande sociale. Les rapports entre cette demande et la domination de la grande entreprise permettent à celle-ci d'articuler les stratégies nationales avec les préoccupations à la fois sociales et industrielles de la nation. C'est pourquoi l'auteur soutient que l'organisation industrielle doit être privilégiée lorsqu'elle accroît la productivité, surtout quand elle est socialement désirée (Villard 1958: 491). De même, l'auteur associe l'évolution rapide de la société à la capacité des grandes entreprises à stimuler, par l'innovation, les dynamiques économiques et sociales. Il écrit :

industries where "competitive oligopoly" prevails are likely to progress most rapidly and that therefore "competitive oligopoly" may well be the best way of organizing industry. The basic point is that progress is likely to be rapid (1) when firms are large enough or few enough to afford and benefit from research and (2) when they are under competitive pressure to innovate - utilize the results of research (Villard 1958: 491).

Les propos de Villard étaient largement influencés par une conjoncture politique qui exigeait une répartition sociale des tâches entre les grandes et les petites firmes. Si les premières devaient promouvoir l'innovation, les secondes devaient se contenter de tirer la pâte. Selon l'auteur, c'était la seule voie devant permettre aux Etats-Unis de continuer à jouer un rôle central dans l'économie du 'monde libre", surtout que cette stratégie

«will maintain our position of leadership in the free world and at the same time will provide the surplus with which to supply aid on an increasing scale to underdeveloped areas of the world that wish to remain free; past standards of research performance have little relevance to what must be achieved in the future» (Villard 1958: 497).

Il semble donc que vers la fin des années 1950, l'innovation dans la grande entreprise était le fondement d'un dynamisme économique par lequel les Etats-Unis cherchaient à se tailler une place de choix sur l'échiquier mondial. Les pratiques des grandes entreprises, qui garantissaient pendant cette période un certain équilibre national et même international, 
révèlent bien un arrangement qui attribuait à la grande entreprise de nombreuses tâches, jusque là dévolues au gouvernement central. Les petites entreprises, les analyses de Villard l'ont confirmé, étaient laissées à elles-mêmes, pratiquement ignorées. Cette tendance allaît se maintenir une partie de la décennie suivante.

Worley (1961) prend la relève êt soutient, lui aussi, que la grande entreprise est généralement plus disposée à innover. 11 montre que les grandes entreprises doivent accorder un intérêt particulier aux activités d'innovation; que l'innovation procède des ressources humaines et financières qu'une entreprise consent à y investir. Selon l'auteur, une entreprise ne s'intéresse à l'innovation que dans la mesure où elle a une structure qui lui permet d'influencer les dynamiques du marché : c'est le cas des grandes entreprises. L'auteur s'explique ainsi :

"We are told that the demands of modern technology are so great that only the large firm is able to spend the sums needed to participate effectively in innovation. We are further assured that we need not fear restraints upon the pace of technology, for the incentives to innovate -largely lacking in industries approximating pure competition - are present in abundance in an oligopolistic structure. Indeed, the large modern concern is likely to feel under considerable pressure to undertake research and to innovate in order not to be outstripped by its rivals, some of whom may have entered the field from other industrial pursuits» (Worley 1961: 183).

Pour Worley, le marché tend à être dominé par un petit nombre de grandes entreprises en concurrence. Pour qu'une entreprise s'impose dans un marché, elle doit être la plus forte et, à cette fin, entreprendre les grands chantiers de la recherche scientifique et de l'innovation. La concentration des activités d'innovation au sein des grandes entreprises paraît donc aller de soi. L'auteur écrit :

«I prefer to share Nutter's view that research has come to be centred in industrial giants partly because the giants were there, ready to welcome the advances of science and the advantages of having scientists in their employ. Restricting the size of these giants by one means or another might merely shift the location of research without seriously hindering it» (Worley 1961: 186).

Worley avance que l'entreprise géante est naturellement la première à pouvoir supporter les frais de la recherche et de l'innovation. Il reprend ici certaines hypothèses avancées par Schumpeter et d'autres. L'argument principal à l'appui de sa démonstration réside dans la 
capacité des grandes firmes à financer leurs activités d'innovation; car elles les financent sans pour autant de ce fait déranger leur expansion. Pour l'auteur, plus une entreprise se concentre sur l'innovation, meilleures sont ses chances de réussir, et de rejoindre le rang des entreprises gagnantes.

Dans la même veine, Hamberg (1964) a démontré que les grandes entreprises innovent davantage, qu'elles s'embarquent dans des activités de R\&D qui assurent un gain de productivité et de prospérité. L'auteur a centré ses idées sur la relation étroite qu'il voit entre la recherche industrielle, principe fondamental de l'innovation, et la taille d'une entreprise (Hamberg 1964 : 62). Selon l'auteur, plus la taille d'une entreprise est importante, plus elle a de potentiel pour innover. La taille d'une entreprise, que l'auteur a mesurée par le nombre total des employés, a une incidence positive sur la réussite de ses activités d'innovation.

De façon générale, cette littérature n'a pas réussi à se distancier des premières hypothèses développées auparavant par Schumpeter, selon lesquelles il revient surtout aux grandes entreprises de s'adonner aux activités d'innovation, et aux activités de R \&D en particulier. Les analyses élaborées par les différents auteurs valorisent les activités et les initiatives menées par les grandes entreprises, et suggèrent que les petites ne doivent pas s'intéresser à des activités qui exigent des ressources humaines et financières souvent énormes, et au-dessus de leurs moyens. Cette perception, qui a dominé à partir du début des années 1960, a animé des débats importants entre scientifiques, chacun s'évertuant à faire ressortir une relation positive entre la taille d'une entreprise et l'innovation. Cette perception va continuer à nourrir la plupart des réflexions jusqu'à la fin des années 1980. Durant cette période, une littérature relativement abondante continue d'avancer que les grandes entreprises, plus que les petites, doivent $s$ 'intéresser aux activités d'innovation.

La plupart des analyses issues de cette perception montrent en fait que les grandes entreprises qui s'impliquent dans ces activités aboutissent à une situation de concurrence, mais oủ un certain équilibre existe entre l'offre et la demande : la conséquence logique est que le marché doit être dominé par des oligopoles. Link (1980) est l'une de ces nouvelles figures. Sur la base d'un échantillon composé de firmes américaines qui opèrent dans le secteur chimique et dans celui des alliages, il a conçu un modèle qui met en relation le taux de rendement des dépenses engagées dans l'innovation, (ici les activités liées à la R\&D) et la taille de l'entreprise, 
exprimée par le nombre total d'employés. Ses résultats indiquent que le taux de rendement estimé des dépenses engagées dans l'innovation s'élève à $78 \%$ pour les grandes entreprises, alors qu'il ne dépasse pas $30 \%$ dans les petites. Ces résultats suggèrent, selon lui, que l'efficacité des activités génératrices d'innovation est fonction de la taille de l'entreprise.

De leur côté, Holemans et Sleuwaegen (1988) ont aussi montré que la taille d'une entreprise, exprimée par le nombre total d'employés, a une influence positive et significative sur les dépenses que les entreprises manufacturières belges consacrent à l'innovation. Il faut préciser ici que Holemans et Sleuwaegen ont mis l'accent sur les activités de R\&D pour évaluer la relation entre la taille d'une entreprise et l'imnovation. Leur analyse a montré que les grandes entreprises sont davantage attirées par l'innovation parce qu'elles deviennent de plus en plus multinationales (Holemans et Sleuwaegen 1988: 375). Il semble donc que le développement des conjonctures internationales impose de nouvelles règles du jeu; l'ouverture d'un marché qui s'internationalise incite les grandes entreprises, plus que les petites, à s'intéresser à l'innovation.

Par ailleurs, Cohen et Klepper (1996) analysent le comportement de 274 entreprises du secteur automobile. La taille des entreprises est mesurée par le nombre d'années de fonctionnement. Les auteurs constatent que, dès que l'entreprise enclenche un processus d'innovation, c'est l'importance de sa taille qui détermine, en fin de compte, ses chances de mieux affronter la concurrence. L'importance de cette taille, considérée en fonction du marché de l'entreprise, agit sur les aptitudes de celle-ci à innover. De même, les auteurs démontrent que le déclin des activités d'une entreprise annonce la régression des retombées de ses innovations précédentes.

Malgré l'importance des changements qui ont touché les structures de l'activité économique, des chercheurs continuent de considérer les grandes entreprises mieux placées pour innover. Les dynamiques économiques de développement évoluent mais la taille d'une entreprise constitue toujours un facteur qui encourage l'innovation.

Klepper et Simons (2000) ont démontré que la capacité d'une entreprise de s'approprier les retombées d'une innovation dépend principalement de sa taille. Ils montrent aussi que les entreprises qui possèdent des expertises variées sont souvent incitées à poursuivre différents 
types d'innovation de produit. L'appropriation croissante des technologies d'information et de communication ne semble pas changer les règles du jeu (Klepper et Simons 2000: 759).

Ainsi, la relation entre la taille d'une entreprise, et son aptitude à innover et à s'imposer dans un marché de plus en plus concurrentiel, paraît désormais inscrite dans un contexte transformé, dominé par l'internationalisation des activités, des valeurs et des normes. Si l'entreprise survit à la concurrence, elle se développe et joue un rôle important dans l'essor de son secteur d'activité. Mais ces conclusions soulèvent d'autres questions, essentielles pour qui veut expliquer les facteurs faisant que la capacité d'une entreprise à innover dépend de sa taille. Blundell et al. ont apporté certaines nuances. Ils ont écrit:

It is often asserted that the superior performance of large firms in innovating is because they have higher cash flows from which to finance investment in R\&D. Our findings suggest that this is not the whole story - dominant firms innovate because they have a relatively greater incentive to do so. Fims with high market shares who innovate get a higher valuation on the stock market than those who do not (Blundell et al. $1999: 551$ ).

Il semble donc que toutes les conjonctures économiques favorisent la domination de la grande entreprise, l'incitent à innover et à vouloir influencer les dynamiques économiques de développement. En raison de certains facteurs, comme les coûts et les efforts exigés, des scientifiques considèrent que seules les grandes entreprises sont capables d'innovation. Dans divers contextes et conjonctures, des chercheurs ont démontré que les grandes entreprises diversifient leurs stratégies d'innovation, expérimentent des procédés et des méthodes nouvelles, tout en cherchant à s'assurer d'un gain de productivité.

Ces vues ne font toutefois pas l'unanimité. D'autres chercheurs, avec des arguments crédibles, sont arrivés à des conclusions contraires, qu'il y a lieu maintenant d'examiner.

\subsection{Pas de relation entre la taille de l'entreprise et l'innovation}

Si les chercheurs ont porté beaucoup d'attention à l'innovation dans les grandes entreprises, ils se sont également intéressés aux réalisations des petites. On l'a vu, Schumpeter a pu affirmer que les petites entreprises peuvent innover. L'évolution des dynamiques économiques de développement a poussé plusieurs scientifiques à scruter l'importance réelle 
de l'innovation dans la petite entreprise. Troublés par la domination des grandes multinationales et fascinés par les efforts des petites entreprises, qui s'enracinent davantage dans leur milieu économique, social et culturel, des scientifiques ont remis en question les hypothèses associant l'innovation uniquement aux pratiques des grandes entreprises. Leurs observations, résumées dans la présente section, indiquent qu'il n'existe pas de relation entre la taille d'une entreprise et l'innovation.

Pavitt et ses collaborateurs (1987) ont analysé au moins 4378 innovations enregistrées entre 1945 et 1983. Ils voulaient comprendre la nature du rapport qui s'établit entre la taille d'une entreprise et l'innovation. Cette réflexion était motivée par le fait que

«the relationship between innovation intensity and firm size was r-shaped with a decline amongst the very large. This pattern of innovative activities was often explained by indivisibilities and risk precluding most small firms, and by monopoly power reducing the pressure on the biggest ones» (Pavitt et al. 1987: 297).

Ces auteurs ont montré que les petites entreprises ont tendance à s'impliquer dans diverses activités d'innovation. Ils ont constaté l'existence d'une vitalité beaucoup plus innovatrice parmi les entreprises de moins de 1000 employés. Ils associent de plus l'innovation, dans cette catégorie d'entreprises, aux conséquences du développement technologique et de ses effets à la fois sur les occasions d'affaires, qui se multiplient, et sur les dynamiques du marché, qui deviennent favorables aux actions innovatrices (Pavitt et al. 1987: 313).

Selon les auteurs, les petites entreprises qui innovent s'épanouissent, car elles améliorent la quantité et la qualité de leur production; ce qui leur permet de concurrencer les grandes sociétés. Leur capacité innovatrice résulte de l'exploitation continue des occasions d'appropriation des technologies sur des marchés de produits connexes. Les PME exploitent les activités de R\&D. Dans ce contexte, les petites entreprises continuent à être innovatrices en fournissant les entrées spécialisées de production, dans la symbiose de grands innovateurs utilisateurs (Pavitt et al. 1987: 313).

Il semble donc que la montée des petites entreprises s'est imposée comme un phénomène économique important, et leur rôle par apport aux activités d'innovation est allé au-delà de la 
simple imitation des innovations mises au point par les grandes entreprises. En effet, les propos de Pavitt et al. font voir que les petites entreprises, tout comme les grandes firmes qui s'internationalisent, s'impliquent plus qu'auparavant dans les activités d'innovation. Ces conclusions traduisent l'importance acquise par les petites entreprises dans les dynamiques économiques.

Dans la même veine, Cohen et al. (1987) ont montré que l'innovation n'est pas réservée aux grandes entreprises. Partant des travaux de Schumpeter, ils ont tenté de saisir les effets de la taille des entreprises sur les activités de R\&D en particulier, et de l'innovation en général. Ils ont réexaminé l'hypothèse de Schumpeter selon laquelle les investissements d'une firme dans les activités d'innovation dépendent de sa taille. Par rapport aux études antérieures, l'approche adoptée est particulièrement originale sur deux points. D'abord, pour les fins de l'analyse, les auteurs ont utilisé les données rassemblées par la Commission fédérale des États-Unis, des données relatives au développement de l'activité économique. Cette démarche méthodologique fournit une vue précise de la relation entre la taille des entreprises et leurs activités d'innovation. Ensuite, on s'est appuyé sur d'autres données utilisées par plusieurs autres auteurs; ce qui a permis à Cohen et al. de comparer leurs conclusions avec celles des autres. Cette tâche a été très utile pour bien voir en quoi les divers secteurs d'activité se différencient en ce qui a traî à l'innovation. Les auteurs concluent qu'en général, il n'existe aucune relation entre la taille de l'entreprise et l'innovation. Ils ajoutent cependant, concernant la $R \& D$, que «a simple regression of $R \& D$ intensity on size measures alone suggests that the size of the firm is positively associated with business unit $R \& D$ intensity, although the effect is quite small» (Cohen et al. $1987: 563$ ).

En général, depuis la deuxième moitié des années 1980, comme l'avaient bien vu Cohen et Pavitt, la littérature scientifique ne pouvait plus attribuer les activités d'innovation uniquement aux entreprises géantes. Les auteurs constatent que les petites entreprises, tout comme les multinationales, contribuent à l'innovation. Ce revirement de cap s'explique, semble-t-il, par la place que les PME ont commencé à occuper dans l'économie et la société.

Au début des années 1990, et surtout avec le développement de la société et de l'économie de l'information, il semble que la taille de l'entreprise n'ait plus aucune influence sur l'innovation. Malecki et Veldhoen (1993) démontrent que même les petites entreprises 
innovent, car elles profitent du potentiel des nouvelles technologies, tout comme le font les grandes entreprises. Mais pour percer, elles doivent diversifier leurs sources d'information: sur le marché, leurs concurrents, leurs fournisseurs, et s'insérer dans des réseaux internationaux.

La plupart des travaux de Malecki et de ses collaborateurs vont dans le même sens, et avancent que l'innovation ne dépend aucunement de la taille d'une entreprise. Les petites entreprises innovent si elles sont guidées par un esprit d'ouverture et d'entrepreneurship, mais principalement si elles sont orientées par une logique qui les met au service de leur milieu; Malecki (1997); Malecki et Gormain (2001).

En somme, les écrits recensés dans cette section récusent la notion d'une relation de cause à effet entre la taille d'une entreprise et l'innovation. Si cette hypothèse s'est avérée dans le passé, l'avènement des technologies de l'information et de la communication, avec l'économie d'information, l'ont rendue caduque. Certains opinent même que plus une entreprise est petite, plus elle s'efforce d'innover.

Depuis le début des années 1980 , les résultats de la recherche scientifique ont montré la grande confusion qui entoure le développement des conjonctures économiques. Les réalités économiques deviennent plus complexes, et il semble que l'on a de la difficulté à saisir la logique sous-jacente au développement.

Quoi qu'il en soit, il apparaît que le nouveau contexte économique et technologique met désormais l'innovation davantage à la portée de la petite entreprise.

\subsection{Une relation négative entre la taille de la firme et l'innovation}

Depuis une quinzaine d'années, une importante littérature scientifique s'est constituée, autour de l'hypothèse postulant qu'il existe une relation négative entre la taille d'une entreprise et l'innovation. Des arguments variés sont invoqués à l'appui de cette hypothèse, et deux variables constituent les principaux instruments de mesure: l'intérêt que les entreprises accordent aux activités de R\&D et les dépenses afférentes à ces activités. Bound et al. (1984) ont trouvé une relation négative entre la taille d'une entreprise et les dépenses que celle-ci 
engage dans l'innovation, les activités de R\&D en l'occurrence. Ils sont partis de la question suivante : Qui fait les activités de R\&D et qui fait breveter? Et ils tirent de leur longue analyse des conclusions importantes. Par exemple, ils démontrent que les activités de R\&D jalonnent le développement de toutes les industries, mais avec une intensité beaucoup plus élevée dans les industries qui utilisent la haute technologie : comme l'industrie chimique, l'industrie des ordinateurs, l'équipement de communication, etc. De même, ils démontrent l'existence d'une élasticité entre les activités de R\&D et la commercialisation des résultats de l'innovation. Is ont également prouvé que la relation entre la taille d'une entreprise et l'innovation n'est pas linéaire; les petites et les grandes entreprises s'intéressent davantage à l'innovation que les entreprises de taille moyenne (Bound et al. 1984 : 48).

Les résultats de cette recherche contredisent les conclusions de tout un pan de la littérature scientifique, et il importait de justifier cette divergence. Selon les auteurs, les faiblesses des études antérieures s'expliquent par le choix des échantillons et des variables pour mesurer l'innovation. Bien entendu, les auteurs n'excluent pas que cette divergence puisse également résulter du changement même de la structure économique et technologique des sociétés étudiées. Pourtant, si cette étude a permis de constater qu'il n'y pas de relation de cause à effet entre la taille d'une entreprise et l'innovation, les auteurs font voir que, dans bien des cas, les petites entreprises, plus que les grandes, ont tendance à innover. Et que si les études scientifiques ne parviennent pas à démontrer la capacitẻ de ces entreprises à innover, c'est tout simplement parce que

"we tend to observe small firms only when they have become "successfull", whereas almost all large firms are publicly traded and will appear in our sample whether or not they have been particularly successful recently in research or innovation» (Bound et al. 1984: 52).

11 apparaît donc que le degré de l'intérêt accordé aux petites comme aux grandes entreprises, dans des champs d'activité où l'innovation est plus visible, influence les résultats de la recherche. Bref, c'est la prise en compte des petites entreprises, avec les grandes, qui semble expliquer que la littérature scientifique admette l'existence ou non d'une certaine relation entre la taille de l'entreprise et l'innovation. 
De leur côté, Acs et Audretsch (1987) et (1988) ont étudié l'effet de la taille de l'entreprise sur l'effort en matière d'innovation, mesuré par le nombre d'innovations réalisées par les firmes du secteur manufacturier américain entre 1978 et 1982. Ils ont montré que les grandes entreprises sont plus performantes dans certains secteurs, notamment ceux qui sont intensifs en capital, alors que les petites entreprises sont plus performantes dans d'autres secteurs, notamment les plastiques et l'électronique. Les auteurs concluent qu'en général, "the empirical results support the modified Schumpeterian hypothesis that the relative innovative advantage of large and small firms is determined by the extent to which a market is characterized by imperfect competition» (Acs et Audretsch 1987: 573). Ils ajoutent: «Industries which are capital-intensive, concentrated, and advertising-intensive tend to promote the innovative advantage in large firms. The small-firm innovative advantage, however, tends to occur in industries in the early stages of the life-cycle, where total innovation and the use of skilled labor play a large role, and where large firms comprise a high share of market) (Acs et Audretsch 1987: 573). Les auteurs ont aussi montré que «innovation activity of small and large firms responds to considerably different technological and economic environments» (Acs et Audretsch 1988: 688).

Par ailleurs, Scherer et Ross (1990) ont avancé que plus la taille d'une entreprise augmente, plus l'incitation à l'innovation risque de diminuer. Les auteurs ont cherché à comprendre les avantages qui poussent une grande entreprise à s'intéresser à l'innovation. Ils ont démontré que l'innovation dans les grandes entreprises est encouragée par la disponibilité des capitaux, l'acceptation du risque et l'ouverture sur un marché important et une vaste clientèle (Scherer et Ross $1990: 652$ ).

Les auteurs soulignent que, malgré ces avantages, les grandes entreprises ont souvent tendance à exagérer le risque qu'elles courent en investissant dans des activités d'innovation, notamment dans les grands laboratoires nécessaires pour la R\&D. Ces raisons seraient les suivantes : perte de temps, complexité des tâches liées aux activités d'innovation, complexité des processus de décision, etc. Les auteurs ajoutent, concernant la possibilité, pour les petites firmes, d'innover : «Even more important, small firms may be more adept at risk taking. Their decisions to go ahead with an ambitious project typically are made by a handful of people who know one another well» (Scherer et Ross 1990:652). Après une longue analyse, les auteurs concluent : 
that no single firm size is uniquely conducive to technological progress. There is a place for firms of all sizes. Technical progress thrives best in an environnement that nurtures a diversity of sizes and, perhaps especially, that keeps barriers to entry by technologically innovative newcomers low (Scherer et Ross 1990 : 654).

L'évolution de la pensée des économistes est perceptible et coïncide généralement avec la diversification et la spécialisation des productions de la nouvelle économie. Pendant des décennies, les chercheurs se sont appliqués à déterminer comment la taille d'une entreprise infuence son innovation. Et selon la méthodologie utilisée et les caractéristiques des économies étudiées, les conclusions ont été souvent divergentes. Toutefois depuis la fin des années 1980 , la recherche accorde un intérêt particulier à la petite entreprise, intérêt qui reflète l'espace qu'elle occupe désormais dans l'économie.

En 1996, Cohen et Klepper publient deux études et arrivent à des conclusions contrastées. La première est une recherche empirique, consacrée aux activités de R\&D et à l'innovation. Ils sont d'avis que la probabilité de réaliser des activités de $R \& D$ augmente avec la taille de la firme, que dans certaines industries, 1 'intensité des activités de R\&D est nécessairement liée à la grosseur de la firme et que la $R \& D$ augmente proportionnellement avec la taille de l'entreprise dans la plupart des industries. Leur étude a démontré que le nombre de brevets ou d'innovations, produits par dollar investi en $R \& D$, tend à décroître lorsque la taille de l'entreprise est moins importante (Cohen et Klepper 1996b: 946). Commentant les résultats de leur travail, les auteurs apportent quelques nuances :

Our results suggest that of these various factors, the advantages of size attributable to cost spreading may be particularly important. As such, the results provide a way of reconciling the long-standing intuition of policy-markets regarding the advantages of large firm size in technological competition with the stylised facts concerning R\&D, innovation, and firm size (Cohen et Klepper 1996b: 948-949).

Dans une autre étude, les auteurs rendent compte de la relation que l'on observe entre la taille d'une entreprise et l'innovation à laquelle elle a recours. Ils montrent que l'entreprise, répondant à la conjoncture et cherchant à s'adapter à la dynamique du marché, est poussée à innover. Ils écrivent : 
If major breakthroughs lend themselves to more discontinuous growth and are more saleable than incremental innovations, this would explain the more casual observation that relative to large firms, small firms appear to be particularly adept at major innovations (Cohen et Klepper 1996a: 242).

De même, les deux auteurs précisent:

Our explanation for findings such as the apparent comparative advantage of either small firms in generating major innovations does not rest on any innate effect of firm size on the productivity of different types of R\&D. To the contrary, our explanation highlights the peril of making inferences about the differential productivity of R\&D in large and small firms from evidence about large and small firms patterns of specialization across different kinds of innovation. In our model small and large firms face exactly the same R\&D project productivity schedules and have the same costs of performing R\&D (Cohen et klepper 1996 a: 242).

Ce que Cohen et Klepper avancent, c'est que l'innovation ne dépend pas de la taille de l'entreprise. Leurs propos montrent que les dynamiques conjoncturelles sont des facteurs décisifs, qui incitent, voire forcent une entreprise à l'innovation. Plutôt que la taille, ce sont d'autres facteurs, liés principalement aux dynamiques existantes qui, comme l'avait constaté Schumpeter, révolutionnent «sans cesse la structure économique de l'intérieur, détruisant sans cesse l'ancienne structure, en créant sans cesse une nouvelle» (Schumpeter 1942 : 83). Pour Cohen et Klepper, comme pour Schumpeter, les dynamiques du marché, tout comme les structures industrielles, subissent des transformations profondes qui se traduisent par des adaptations organisationnelles et des initiatives par lesquelles les entreprises, indépendamment de leur taille, cherchent à se ménager une place de choix. II apparaît par ailleurs que Cohen et Klepper ne soutiennent pas l'hypothèse schumpeterienne voulant que le développement économique résulte largement de la diffusion d'innovations radicales à un grand nombre d'entreprises, qui imitent les grandes entreprises à l'origine de ces innovations.

Dans un autre contexte, Klepper et Simons (2000) ont tenté de saisir les principales caractéristiques de l'évolution de l'industrie automobile, bouleversée par les changements technologiques. Ils ont étudié les effets de ces changements, particulièrement l'innovation dans les entreprises, à l'aide d'indicateurs caractéristiques comme la taille, la localisation, l'affiliation à des réseaux de distribution et les choix technologiques. Ils ont démontré que l'importance d'une entreprise a un effet positif sur sa capacité à innover et à survivre face à 
une âpre concurrence. Ils ont constaté de plus que l'innovation des entreprises, dans une industrie qui subit les effets d'un changement technologique, a des retombées importantes sur la taille des entreprises, leur survie et éventuellement leur transformation en oligopole (Klepper et Simons 2000: 759).

Clairement, il apparât que la recherche scientifique récente a reconnu la place importante occupée, dans le développement économique, par la petite entreprise, aux côtés de la grande, chacune nécessaire dans un créneau donné. Aux grandes, tout en convenant de leur rôle stabilisateur du marché et de leur capacité à innover, on reproche souvent leur insensibilité par rapport aux répercussions sociales et environnementales de leurs activités. Chez les petites, on note qu'elles prennent davantage en considération les besoins des acteurs locaux et des collectivités, et qu'elles s'adaptent plus facilement à une conjoncture mouvante. Ces observations, reflet des aperçus de la plupart des auteurs précités, trouvent un écho dans les écrits émanant du GREMI : que les petites entreprises qui savent s'insérer dans des réseaux appropriés, innovent, tout comme les grandes.

Peut-on affirmer aujourd'hui qu'un rapport existe entre l'importance de la taille d'une entreprise ef l'innovation que celle-ci génère ? Comme en fait foi le présent chapitre, les opinions divergentes reflètent le parcours historique de l'industrie et de l'économie, en même temps qu'un cheminement de la pensée et une évolution dans la façon d'appréhender les situations étudiées. Une lecture attentive de la littérature pertinente montre que le fonctionnement et le rôle de l'entreprise, petite ou grande, ont bien changé depuis les premiers travaux de Schumpeter. Si certains ont vu, pendant un temps, la grande entreprise comme le moteur quasi unique de l'innovation, les avis sont plus nuancés aujourd'hui et plusieurs inclinent à croire qu'il n'y a guère de différence quant à l'innovation, entre des firmes de tailles diffërentes.

Dans les études récentes, on a cherché à raffiner les critères, on a utilisé un plus grand nombre de variables et on a tenu compte davantage du contexte où évolue l'entreprise. Mais aussi ce qui a grandement changé la donne, c'est l'avènement des nouvelles technologies numériques, qui a favorisé la prolifération des PME et a permis à plusieurs d'entre elles de devenir des pépinières d'innovation. Si bien que la plupart des chercheurs considèrent maintenant que l'innovation peut survenir dans les petites comme dans les grandes entreprises. Certains 
toutefois demeurent d'avis que l'innovation, dans les grandes entreprises, a des effets plus importants que celle produite par les petites. Par exemple, Blundell et al. ont écrit : «leading firms have a systematic tendency to produce innovations that are intrinsically of higher quality than smaller firms» (Blundell et al. 1999: 550).

Cette affirmation de Blundell est peut-être vraie d'un point de vue macroscopique, ou lorsqu'une grande firme débouche sur une percée scientifique vraiment fondamentale après des années de recherche. Cela est toutefois relativement rare. Ce qui est plus fréquent, mais moins spectaculaire, c'est la mise en application d'une idée nouvelle, qui réforme les façons de produire ou met au point de nouveaux produits.

En définitive, l'imagination, l'ingéniosité, l'audace ne sauraient être l'apanage d'une catégorie unique. Les firmes, petites ou grandes, qui ont innové, ont su saisir l'opportunité quand elle s'est présentée. À la question de savoir si et comment la plus ou moins grande taille d'une entreprise influe sur son innovation, une réponse catégorique parait improbable. L'histoire économique, comme le reste, n'est pas statique. Le savoir s'accroît, la société évolue, les besoins changent, de même que le marché. Toutes les entreprises sont entraînées dans ce mouvement, qu'elles peuvent parfois pressentir, au moins s'y adapter. Voilà pourquoi, malgré les efforts qui ont enrichi la littérature sur cette question, des zones d'incertitude persistent, que la recherche continuera à tenter d'élucider.

Il apparaît toutefois que quiconque veut appréhender la relation pouvant exister, entre la taille d'une firme et l'innovation, doit prendre en considération non seulement les caractéristiques de la firme, mais également la conjoncture du moment et son environnement territorial et social.

\section{Conclusion}

Il a été montré dans un premier chapitre qu'une appropriation convenable des technologies de pointe peut faire la différence entre les entreprises et entre les territoires; que cette appropriation influence les pratiques entrepreneuriales, que ses effets ne se sentent pas de la même manière dans tous les territoires et chez toutes les entreprises. Ce qui nous a permis de conclure que les TIC, à elles seules, n'empêchent pas le développement inégal des conditions 
économiques, sociales et institutionnelles qui dominent aujourd'hui. Nous avons ensuite montré que l'État et le secteur privé jouent des rôles variables, mais importants dans l'appropriation et l'usage des TIC. Le deuxième chapitre a fait voir que le choix des entrepreneurs de s'affilier à des réseaux d'entreprises peut favoriser l'émergence de pratiques novatrices. Dans une économie dite d'information, les réseaux d'entreprises favorisent la circulation des connaissances et de l'information stratégique, lesquelles, à leur tour, encouragent l'apprentissage, la concertation et le développement des dynamiques d'innovation. Il apparaissait alors indiqué d'examiner comment la taille plus ou moins importante d'une entreprise influe sur sa contribution à ces dynamiques. Le présent chapitre a donc été consacré à cette question.

Traiter des effets de la taille des entreprises sur le changement technologique et sur l'innovation n'est pas une problématique nouvelle. Une littérature abondante et diversifiée s'y est intéressée. Nous avons donc remonté aux origines des débats, lorsque Schumpeter voulait comprendre les liens existant entre la taille des firmes et l'innovation. L'auteur a décrit l'influence des structures organisationnelles non seulement sur les pratiques entrepreneuriales et sur les activités économiques en général, mais surtout sur les compétences et les enjeux qui incitent une entreprise à innover. L'analyse de la littérature que nous a léguée Schumpeter révèle un paradoxe de prime abord étonnant : il lie l'innovation tantôt à l'entreprise géante et tantôt à l'entreprise de petite taille. Il en est résulté une certaine confusion parmi les scientifiques qui se sont intéressés aux ouvrages de Schumpeter. Toutefois le paradoxe n'est qu'apparent; ayant analysé deux contextes différents, Schumpeter a abouti à des conclusions opposées, qui se comprennent lorsque prises dans leur contexte respectif. C'est dire que, dépendamment de la conjoncture et de la structure économique en place, l'innovation peut être l'œuvre d'une grande ou d'une petite entreprise. L'apport de la pensée de Schumpeter est d'avoir ouvert aux chercheurs des pistes de recherche très fertiles. Cet apport est majeur.

Les successeurs de Schumpeter ont avancé des idées qui oscillent entre trois thèses principales : l'innovation s'accroit avec la taille de la firme; il n'y a pas de relation entre la taille de l'entreprise et l'innovation; il y a une relation négative entre la taille de la firme et l'innovation. Dans le présent chapitre, nous avons vu que chacune de ces thèses se fondait sur des données empiriques qui lui donnaient une certaine légitimité scientifique. Se basant sur de bons arguments, les tenants de chaque thèse expliquent l'occurrence d'innover par l'ensemble 
des schémas d'action considérés. Les conclusions varient, dépendamment du contexte économique et social en place et des outils méthodologiques et scientifiques utilisés par les chercheurs.

La littérature récente se préoccupe peu de l'influence de la taille des firmes sur linnovation; elle s'intéresse davantage aux dynamiques internes des entreprises et à leur aptitude à résoudre les problèmes complexes nés de la conjoncture où elles évoluent (Dosi et al. 2003 et 2003a). Selon ces auteurs, lorsqu'un acteur est confronté à une situation problématique, il tend à réagir par une série de démarches de consultation, de coopération et de coordination, démarches qu'il juge importantes pour cerner la solution appropriée. Dosi et coll. suggèrent plutôt un modèle qui permet de décomposer un problème en éléments distincts, de façon à en identifier les facteurs d'intrication et les solutions possibles. C'est donc à partir de cette segmentation du problème qu'il y a lieu de chercher des solutions. Ainsi, au lieu d'insister sur les stratégies de coopération et de coordination des entreprises, il semblerait plus utile, pour le chercheur, de voir comment ces entreprises s'organisent, s'ajustent, modulent leurs actions, leurs réactions, tout en cherchant, chacune pour son compte, à s'adapter aux contraintes du moment.

En définitive, on ne peut plus associer l'innovation à la taille d'une entreprise. Avec la tendance des entreprises à se grouper en réseaux et la domination de l'économie dite d'information, l'innovation a pris des dimensions nouvelles, qui en font d'abord et avant tout un processus lié aux interactions de l'entreprise avec les autres organisations de son milieu. Nous avons ainsi été amené à tenter de préciser le rapport existant entre la taille d'une entreprise, petite ou grande, et son aptitude à s'affilier à un réseau, à traiter convenablement l'information stratégique, à examiner les pistes qui s'ouvrent devant elle et enfin à faire des choix qui augmentent sa productivité et sa compétitivité. L'innovation ne dépend donc plus de la taille de l'entreprise, mais plutôt de son habileté à s'adapter aux exigences de la conjoncture existante. Ces exigences étant en bonne partie conditionnées par les composantes du territoire et du milieu, il convient de voir comment ces composantes influencent l'innovation dans les entreprises. C'est le sujet du chapitre suivant. 


\section{CHAPITRE 4}

\section{L'INFLUENCE DU TERRITOIRE ET DU MILIEU}

La recherche relative au développement régional fait naturellement appel à des notions telles que le territoire, le milieu, l'innovation, dont plusieurs chercheurs ont noté l'influence sur le dynamisme d'une région. Afin de rendre compte de la réalité actuelle, il y a lieu également de considérer l'influence des TIC dans le fonctionnement des entreprises et de la société. Ces notions sont tour à tour examinées dans le présent chapitre : le territoire et le milieu; les liens existants entre l'innovation, le territoire et le milieu; le rôle des TIC dans l'établissement et le devenir de ces liens; comment la conjonction de ces notions a permis la conception d'un appareil explicatif mobilisateur.

La première section de ce chapitre est consacrée au milieu innovateur, ainsi qu'aux systèmes national, régional et spatial d'innovation, en vue d'en dégager les fondements conceptuels. La seconde partie traite de distance et de proximité, telles qu'influencées par la donne conjoncturelle. Cette réflexion paraît utile à la formulation d'un cadre conceptuel nécessaire à la poursuite du présent travail qui porte sur les TIC et leurs effets sur les dynamiques d'une société et d'une économie d'information. 


\subsection{Milieu innovateur et systèmes d'innovation}

Dans la décennie 1980 et le début de la suivante, les dynamiques économiques et sociales de développement de certains territoires ont suscité l'intérêt de plusieurs scientifiques éminents. La notion de milieu innovateur s'est imposée comme outil théorique indispensable à la compréhension du développement inégal des territoires. Elle a été formulée pour rendre compte de l'existence, dans certains territoires, de liens forts entre l'innovation, la croissance et la prospérité. Pour expliquer des réalités économiques et sociales de plus en plus complexes, d'autres idées se sont ajoutées, notamment le concept de système d'innovation.

\subsubsection{Le milleu innovateur}

La notion de milieu innovateur a beaucoup été utilisée pour apprécier la vitalité de certains territoires. Elle est ici examinée dans l'optique du GREMI et par rapport à l'utilisation des technologies de l'information et de la communication.

Dans les années 1980, Philippe Aydalot procède à la création du Groupe de Recherche Européen sur les Milieux Innovateurs (GREMI). Cette initiative crée un vaste programme de recherche sur les milieux innovateurs. L'hypothèse à la base de ce programme met en relief le «rôle déterminant joué par les milieux locaux comme incubateurs de l'innovation, comme prisme à travers lequel passeront les incitations à l'innovation et qui donnent sur le terrain son visage à celle-ci ; l'entreprise n'est pas un agent innovateur isolé ; elle est partie du milieu qui la fait agir» (Aydalot 1986:11). Cette hypothèse a soulevé plusieurs questions concernant le potentiel des parties d'un milieu local et leur capacité à innover. Les principales questions ont trait aux points suivants : «-l'appréciation des trajectoires technologiques des entreprises; l'évaluation des facteurs locaux d'innovation; - le rôle de l'extérieur dans l'entraînement de l'innovation ; - les synergies locales» (Aydalot 1986:13). Il semble dés lors que par ce vaste programme on veut mettre l'accent sur les acteurs de développement, et surtout sur leur aptitude à profiter des synergies locales. L'initiative des acteurs locaux se trouve donc au centre des préoccupations scientifiques. Et l'on mise davantage sur ces initiatives pour comprendre «l'émergence d'un esprit innovateur local et dans la mise à disposition des connaissances technologiques, dans leur financement» (Aydalot 1986:5). 
On voit donc que la création du GREMI ouvre deux perspectives. D'abord, celle des acteurs de développement, car il suffit d'identifier les principaux acteurs de développement, de s'assurer qu'ils profitent des outils mis à leur disposition et susceptibles de servir de support aux activités innovatrices. Tout en profitant de ces outils, les acteurs participent à la création d'un cadre général porteur d'un nouveau potentiel, qui permet l'emboîtement social, culturel et entrepreneurial des pratiques.

L'autre perspective offerte par la création du GREMI est celle qui s'ouvre devant les chercheurs, appelés à cerner les dynamiques économiques et sociales de développement en partant des interactions et des interdépendances entre acteurs qui partagent un même milieu. D'où l'importance de la littérature produite dans ce cadre.

Aydalot et Keeble publient en 1988 un ouvrage important: «High Technology Industry and Innovative Environments : the European Experience»; cette publication confirme l'hypothèse selon laquelle le contexte local joue un rôle crucial dans les processus d'innovation. L'idée centrale qui se dégage de ce travail est l'identification, de plus en plus claire, d'une régulation locale originale, émergeant des structures et des actions locales, qui crée les conditions favorables au développement et à la prospérité. Les auteurs parlent des «grappes locales» ou des systèmes locaux de production, afin de mettre en valeur cette idée (Aydalot et Keeble 1988).

Les premiers travaux qui ont émané du programme de recherche mis sur pied par le GREMI étaient prometteurs et ont provoqué des débats animés entre les scientifiques. De même, ils ont largement inspiré les politiques de plusieurs gouvernements. Les principales contributions qui ont assuré la continuité de ce programme sont présentées dans les paragraphes suivants.

En 1991, avec son ouvrage : «Innovation Networks : Spatial Perspectives», Camagni renforce certaines idées et hypothèses contenues dans le programme de recherche lancé auparavant par Aydalot. En quelque sorte, il en est la continuité logique; la pensée de Camagni ne se contente pas seulement de montrer que les activités économiques se développent selon les modèles habituels (polarisation, division spatiale du travail, etc.), mais démontre aussi que ces modeles se renouvellent et s'enrichissent selon les conjonctures locales et régionales. C'est donc dire que les synergies territoriales et les impulsions internes et externes jouent un rôle 
crucial dans l'évolution des dynamismes que génèrent les divers milieux (Camagni 1991). L'une des principales contributions de l'auteur est l'adoption d'une approche centrée principalement sur les problématiques du milieu, lesquelles engendrent à la fois information et incertitude, influençant de ce fait les coûts de transactions. Selon l'auteur, les entreprises qui font face à l'incertitude et au manque d'information recourent souvent aux composantes de leur milieu, pour profiter du soutien de l'organisation, tacite ou explicite, d'interdépendance fonctionnelle et informationnelle des acteurs locaux. L'on peut ainsi tirer profit des comportements liés à la recherche, la transmission, la sélection, la transcription, la transformation et le contrôle de l'information (Camagni 1991).

Se basant sur l'information et sur son rôle dans la gestion des opérations quotidiennes d'une entreprise, l'auteur définit les principaux paramètres de l'innovation, qu'il présente d'ailleurs comme le résultat d'une combinaison originale des aptitudes technologiques de l'entreprise avec les exigences de son marché. L'innovation est le résultat des interactions entre ces paramètres et ces exigences. Il faut donc souligner que ces interactions renvoient aux interdépendances des éléments du marché, d'abord d'un milieu local, mais sans pour autant exclure l'influence d'éléments et de facteurs pouvant provenir de l'extérieur. Dans le cas d'un milieu innovateur, l'enjeu est donc de savoir toujours mieux profiter à la fois d'organisations territoriales diversifiées et des apports des réseaux intra et extra-territoriaux.

Le travail de Camagni, évoqué ci-dessus, a enrichi la réflexion autour d'hypothèses touchant le milieu et sa capacité à influencer les dynamiques économiques et sociales de son développement. L'évolution de la conjoncture, au début des années 1990, et les exigences qui en découlaient, ont amené Camagni à élargir les frontières fixées par Aydalot, ce qu'il a réalisé avec une rare habileté.

Le début des années 1990 coïncidait donc avec l'émergence de nouvelles dynamiques économiques et sociales, qu'il fallait reconnaitre et théoriser. Maillat et Perrin (1992) consacrent alors, aux entreprises innovatrices et au développement territorial une réflexion signalée. Plusieurs chercheurs étaient invités à traiter des questions cruciales que pose le développement économique à certains territoires. Maillat résume bien ces questions : 
pourquoi certains territoires sont-ils plus innovateurs que d'autres ? Comment caractérise-t-on les milieux innovateurs ? Comment les analyses en termes de milieux innovateurs peuvent-elles contribuer à réinterpréter les théories du développement spatial et à donner de nouvelles orientations à la politique régionale ? Pourquoi ces approches sont-elles particulièrement adaptées à la période de mutations techno-organisationnelles que nous traversons ? (Maillat ef Perrin $1992: 3$ ).

L'une des idées maitresses de cet ouvrage est de considérer l'entreprise comme une microorganisation; tandis que ses environnements local et régional sont présentés comme des macro-organisations (Maillat et Perrin 1992: 229). Il y a là une nouvelle initiative, pour mieux comprendre les modalités territoriales de la dynamique industrielle qui orientent le développement d'un territoire. Dans cet ouvrage, Maillat et Perrin ont confronté les études portant sur l'élaboration des imnovations et leurs effets sur l'environnement local des entreprises, dans une dizaine de régions d'Europe et des Etats-Unis. Ils sont «intéressés par un processus global de structuration-déstructuration dont la logique territoriale se combine avec celle des processus industriels mais qui ne se confond pas avec elle» (Maillat et Perrin 1992 : 230). Cette confrontation permet de dégager une relation originale entre l'entreprise et son environnement, montrant que les innovations et leur impact sont, d'une certaine manière, coproduits par les entreprises et par les constituantes de leur environnement local-régional.

On voit ici une manière originale, qui permet d'adapter aux exigences de nouvelles conjonctures les principes apportés auparavant dans le cadre du programme de recherche du GREMI. Les conclusions sont donc dictées pardes exigences de mise en valeur des territoires, de leurs ressources, de leur histoire et surtout de la capacité des acteurs locaux à mâtriser l'évolution des dynamiques techno-productives en place. C'est dans la conclusion de cet ouvrage que Perrin apporte certains éléments de réponse à la problématique des liens entre l'innovation et les dynamiques propres à l'entreprise et à son environnement. II écrit :

notre argument est que la dynamique socio-économique procède à la fois par création de technologies et par construction de "territoires" et que ces deux processus sont étroitement liés de sorte que, de même que la dynamique industrielle a un effet déterminant sur le développement des systèmes locaux régionaux, ce qui est le plus souvent admis, de même, lorganisation territoriale des activités productives contribue de manière tout aussi importante à la progression de leurs capacités, notamment dans l'ordre technique et technologique, ce qui est rarement reconnu (Maillat et Perrin 1992 : 229). 
On le voit, le milieu innovateur reste toujours au centre des préoccupations scientifiques. L'accent est mis tantôt sur les composantes internes de ce milieu, tantôt sur les relations que ces composantes entretiennent avec leur environnement, proche ou éloigné. Dans la même foulée, Maillat, Quévit et Senn (1993) avancent une définition plus précise du milieu d'innovation. Selon eux,

un milieu innovateur se caractérise par lintégration de dynamiques internes et de changements survenus à l'extérieur. Dès lors: le milieu est innovateur lorsqu'il est capable de s'ouvrir à l'extérieur et d'y recueillir des informations, voire des ressources diverses. Le milieu innovateur par essence s'ouvre sur la diversité de l'environnement en s'enrichissant dans la réceptivité au changement; le milieu est innovateur lorsque ses ressources sont organisées, coordonnées et mises en relations par des structures économiques, culturelles et techniques qui rendent les ressources exploitables pour de nouvelles combinaisons productives (Maillat et al. $1993: 13)$.

Les auteurs ont aussi cherché à comprendre les relations entre les réseaux d'innovation et les milieux innovateurs. Leur souci est de mieux répondre à deux problématiques importantes, traitées chacune dans une partie de leur ouvrage. Une première partie est consacrée aux interactions que l'on tient responsables du développement des milieux et qui font que des réseaux simples deviennent des réseaux d'innovation. La deuxième partie traite des problèmes relatifs à la transformation des milieux et des réseaux respectivement en milieux innovateurs et en réseaux d'innovation. Plus précisément, les auteurs ont retenu une problématique générale qu'ils ont articulée autour de trois champs d'interrogation:

- le réseau d'innovation: quelle est sa genèse, son architecture, son mode de fonctionnement, sa stratégie, enfin son évolution? Comment se développent les interactions entre les différents acteurs ? Quelles règles et quelles normes gèrent leurs relations ? Comment les processus d'apprentissage se développent-ils à lintérieur du réseau dinnovation? les relations entre le milieu et le réseau dinnovation: quel est le rôle du milieu dans le réseau d'innovation et sur son évolution? les effets du réseau d'innovation sur le milieu: quel est limpact sur le milieu local des processus d'apprentissage qui sont engendrés par le réseau d'innovation ? Comment ceux-ci contribuent-ils à l'accroissement des capacités créatrices du milieu ? (Maillat et al. 1993 : 6).

Partant de ces questions et des exigences d'ordre à la fois conjoncturel et structurel, les auteurs ont mis en évidence l'aptitude des acteurs à promouvoir le développement spatial, ce 
qui témoigne de la capacité des territoires et des régions en général à intégrer dans leur tissu productif les nouveaux paradigmes du système techno-industriel. Comme prometteur de l'avantage technologique, le rôle des réseaux d'innovation est souligné. Selon les auteurs, les formes variées de complémentarité et de spécialisation technologique, par exemple, sont nécessaires au développement des dynamiques d'un territoire. De même, la diversité des modalités organisationnelles constitue une richesse importante, qui permet aux acteurs de mieux mâtriser l'incertitude souvent associée aux processus d'innovation. Dans un milieu innovateur, la combinaison de ces éléments crée les conditions favorables au développement de synergies variées, incitant les acteurs d'un milieu à tirer avantage des liens, formels et informels, ainsi créés.

Au cours des années 1990, le GREMI a diversifié ses programmes de recherche et a profité de la collaboration de scientifiques avertis. Les thèmes retenus montrent l'importance, la diversité et la richesse des problématiques étudiées. Par exemple, certains programmes ont été affectés à l'étude du milieu, des réseaux et des effets des réseaux sur les dynamiques des milieux (GREMI 3 et GREMI 4). Le programme GREMI 5 traitait d'une question centrale : comment le milieu innovateur se différencie-t-il de la ville ? Le programme GREMI 6 devait clarifier en quoi le concept de milieu innovateur peut-il s'appliquer à des ressources non industrielles : élargissement de la notion de milieu.

Ce programme marque un tournant important dans les travaux de cette école de pensée. Au lieu de se concentrer sur les milieux innovateurs, qui se démarquent par leur plus grande capacité de s'auto-organiser à travers diverses formes de «partenariat», GREMI met en place une multitude de modalités d'ajustement mutuel du savoir et des savoir-faire des acteurs (Maillat 1998). Ce programme défend l'idée que l'analyse ne doit pas se concentrer uniquement sur les dimensions technologiques et industrielles des activités, mais doit aussi tenir compte de la diversité et de la richesse des ressources naturelles et culturelles dont dispose un territoire.

En général, depuis les premiers travaux d'Aydalot jusqu'aux travaux les plus récents, l'accent est mis sur certaines constantes, qui montrent en quoi et comment les dynamiques propres aux milieux et aux territoires contribuent à l'innovation. La formulation de ces constantes tient compte des entreprises actives dans divers milieux, lesquels sont enrichis d'une multitude 
d'éléments qui, si bien organisés et territorialisés, contribuent efficacement à la production et à la diffusion de l'innovation.

Les travaux rattachés au GREMI, fin des années 1990 et début des années 2000, constituent un apport scientifique important; ils aident à mieux comprendre la diversité des dynamiques économiques et sociales de développement, devenues plus complexes que ce qu'elles étaient pendant les années 1980. Ces travaux ont mis des outils scientifiques de grande utilité théorique et pratique au service des acteurs de développement et sont devenus indispensables pour qui veut comprendre le processus du développement dans un territoire. Par exemple, la distinction faite souvent, et surtout depuis la fin des années 1990, entre un milieu innovateur et une ville correspond à l'observation que l'innovation ne se limite pas qu'aux centres urbains. Cette distinction reconnaît, explicitement ou non, que l'innovation n'est pas propre aux dynamiques urbaines, et cela même si celles-ci sont souvent plus complexes et encouragent le développement d'interactions plus denses (Crevoisier et Camagni 2000). Comme le montre le programme de recherche de GREMI 6, les richesses d'un territoire ne peuvent pas être uniquement économiques; elles sont d'abord humaines; elles sont aussi naturelles et environnementales; ce dont il faut tenir compte pour évaluer la capacité d'un territoire, qu'il soit urbain ou rural, central ou périphérique, à innover.

\subsubsection{Le système national d'innovation}

La notion de système national d'innovation est particulièrement associée aux travaux de Freeman (1987), Lundvall (1992), Nelson (1993) et Edquist (1997). Plus spécifiquement, on attribue l'origine de ce concept à la réflexion de Freeman qui, en 1987, publie un travail signalé sur des problématiques liées à la compétitivité économique au Japon. Cette notion a par la suite été enrichie par plusieurs contributions scientifiques.

Il existe en fait plusieurs définitions d'un système national d'innovation. Pour Freeman un système national d'innovation renvoie au réseau des établissements publics et privés, dont les activités et les interactions lancent, importent, modifient et diffusent les nouvelles technologies (Freeman 1987). De son côté, Lundvall (1992) définit un système national

d'innovation comme un ensemble d'éléments entre lesquels se développent des rapports, leur permettant d'interagir dans la production, la diffusion et l'utilisation des produits nouveaux. 
Un système national d'innovation se distingue aussi par une présence accrue de la connaissance économiquement utile. Selon Lundvall, ces éléments sont généralement localisés et enracinés à l'intérieur des frontières d'un État. Par ailleurs, Nelson et Rosenberg (1993) présentent un système national d'innovation comme l'ensemble des établissements dont les interactions déterminent l'innovation des sociétés nationales.

Nelson (1993) présente le système national d'innovation en mettant l'accent sur la coexistence remarquable des secteurs public et privé, qui participent directement à la promotion de l'innovation, par l'intermédiaire des universités et des laboratoires du gouvernement. Ils y participent également indirectement, en créant les structures qui favorisent le développement de divers processus d'innovation et en participant à la promotion de l'éducation, des infrastructures de transport, etc. De même, ce système national est encouragé par l'adoption d'une politique commerciale appropriée, comprenant notamment des mesures fiscales et monétaires nécessaires à la promotion des exportations. De leur côté, Niosi et collaborateurs (1993) indiquent que le système national d'innovation englobe l'ensemble des interactions des sociétés privées et publiques (grandes ou petites), des universités, et des organismes gouvernementaux visant la production de la science et de la technologie dans les frontières nationales. Entre les composantes de ce système national s'établissent des interactions qui peuvent alors être techniques, commerciales, légales, sociales ou financières. L'objectif de ces interactions est le développement, la protection, le financement ou la régulation des nouvelles connaissances scientifiques et technologiques.

Il semble donc que la notion de système national d'innovation recouvre avant tout la localisation des activités économiques, sociales, politiques, dans un cadre territorial très large. Il est toutefois difficile de préciser les principaux indicateurs qui peuvent servir à mesurer la performance d'un système national d'innovation et d'en dégager les forces et les faiblesses.

La littérature scientifique propose néanmoins certaines pistes. Par exemple, on peut s'intéresser à la performance des établissements en établissant des rapports entre les entrées de base (dépenses, personnel) et les sorties (brevets, publications, rapports internes, innovations, nouveaux produits). On peut ensuite analyser leur interaction par l'intermédiaire de variables telles que la taille, la propriété et la distribution nationale des biens et services. 
Il faut préciser que plusieurs études et analyses ont tenté de lier le développement d'un système national d'innovation aux capacités d'un cadre territorial à s'approprier convenablement les TIC. Par exemple, Edquist et Lundvall (1993) avancent qu'un système national d'innovation peut être constitué par les établissements et les structures économiques qui affectent le taux et la direction du changement technologique de la société.

De son côté, Metcalfe (1995) constate qu'un système national d'innovation est constitué d'un ensemble d'établissements et d'institutions distinctes, qui contribuent conjointement et individuellement au développement et à la diffusion des TIC, et qui constituent le cadre dans lequel les gouvernements conçoivent et mettent en application des politiques pouvant stimuler l'innovation. Le système national d'innovation se présenterait donc comme ce cadre qui sert à créer, stocker et transférer la connaissance, le savoir-faire et les nouvelles technologies (Metcalfe 1995). L'action d'un ensemble d'établissements dynamise ce cadre: les sociétés privées, les universités et d'autres établissements éducatifs, laboratoires publics de recherche, consultations privées, sociétés professionnelles, réseaux d'entreprises, associations de recherche industrielle. Ces établissements apportent des contributions complémentaires, mais qui diffèrent de manière significative en ce qui concerne la motivation et la diffusion de la connaissance qu'ils créent (Metcalfe 1995).

On voit donc que les scientifiques mettent davantage l'accent sur les institutions et sur leurs aptitudes à promouvoir l'innovation. Effectivement, Edquist constate que les principales caractéristiques d'un système national d'innovation sont l'existence d'un cadre institutionnel approprié, accompagné d'un système de production important (Edquist 1997:15). Que les institutions d'un territoire national soient formelles ou informelles, elles incitent les acteurs à entreprendre, leur fournissent l'information et les ressources nécessaires afin de réduire l'incertitude et atténuer les effets néfastes de conflits éventuels (Edquist 1997: 15). Manifestement, on insiste toujours non seulement sur l'existence ou non d'institutions à vocation nationale, mais surtout sur leur capacité à influencer positivement les comportements des acteurs, à encourager l'innovation. C'est en prolongement de ces idées qu'Edquist précise le sens de l'innovation: «Innovations are new creations of economic significance. They may be brand new but are more often new combinations of existing elements. Innovations may be of various kinds (e.g., technological and organizational) (Edquist 1997: 1). Ainsi définie, cette notion éclaire la façon d'appréhender un système d'innovation: 
"A system of innovation should be looked upon as a 'whole' because any of its elements are - more or less closely - related to each other. Otherwise, there would be no 'system'. But it is also sometimes necessary to deal only with parts of the system - one at a time or a few in relation to each other. Hence, it may sometimes be necessary to restrict the analysis to various subsystems of a system of innovation. [...] To study only one subsystem can also contribute to the creation of a more coherent and sharp 'scientific language' appropriate for dealing with elements like technological development, diffusion of technology, the mergence and diffusion of organizational forms, education and training, as well as institutional changes related to these spheres» (Edquist 1997:18).

II semble donc que, pour mieux comprendre le fonctionnement d'un système national d'innovation, il est nécessaire d'analyser «all important economic, social, political, organizational and other factors that influence the development, diffusion and use of innovations» (Edquist 1997:14).

Il appert toutefois qu'on ne peut se contenter d'observer le fonctionnement d'un système national pendant un laps de temps relativement court pour en tirer un jugement global sur le long terme. Au contraire, la continuité dans le temps des interactions entre les institutions, de même que de l'innovation, est une condition nécessaire d'un véritable système national d'innovation. Un système doit disposer des qualifications, de l'expérience et de l'effort que les entreprises utilisent pour acheter, employer, adapter, améliorer et créer des technologies. Lall (1999) constate que l'entreprise individuelle est l'unité fondamentale de l'activité technologique, mais considérée isolément, elle ne peut pas avoir une grande influence. C'est donc son intégration dans un système national qui donne de la valeur à ses activités. L'activation dans le temps des composantes de ce système national influence positivement ou négativement la coopération, la coordination et l'échange d'information entre les entreprises d'un même système national. Dans cette perspective Niosi (2002) a suggéré les causes de dysfonctionnement d'un système national. Il conclut : «At the basis of both inefficiency and ineffectiveness one can probably find the weight of past decisions, path-dependent results of organizational and technological trajectories stemming from localized learning and initial conditions» (Niosi 2002: 300).

D'autres chercheurs tentent de donner un nouveau souffle à ce concept dont l'utilité et la pertinence scientifique n'est plus à démontrer. Par exemple, Lundvall (2004) évoque l'effervescence qui a accompagné la présentation par Freeman du concept de "système 
national d'innovation', et il est d'avis que le travail de cet auteur continue d'exercer sur les chercheurs et sur les politiciens une grande influence. À la lumière de la conjoncture actuelle, Lundvall propose d'utiliser ce concept de système national d'innovation, qui peut être utile pour expliquer les liens forts pouvant exister entre les politiques commerciales et le système national en place. Selon cet auteur, un système national d'innovation n'est plus perceptible que dans les situations où les économies nationales, en plus de stimuler l'innovation, s'imposent dans la conjoncture internationale. Pour introduire son travail, l'auteur écrivait : «I shall challenge the idea that "innovation system is not a theorical concept" and show that current innovation system analysis still can learn from him (Freeman) when it comes to linking together trade policy and national innovation systems» (Lundvall 2004: 532).

Mise en contexte par Lundvall, les réflexions consacrées à ce concept l'ont enrichi et l'ont adapté à des conjonctures variées. On l'a utilisé pour rendre compte des dynamiques de développement de pays tels que les Etats-Unis, le Japon, la Russie, l'Afrique du Sud, la Chine, l'Inde, mais aussi de certains pays plus petits, comme la Finlande. La diversité de ces contextes explique la multitude des définitions que les scientifiques ont données à ce concept. Pourtant, la plus grande utilité actuelle de ce concept réside dans son aptitude à rendre compte des dynamiques de développement propre aux conjonctures marquées par l'internationalisation des enjeux et par la globalisation des économies. Selon l'auteur, ce concept est désormais intéressant pour comprendre comment l'innovation se propage dans un espace national, sous la pression des facteurs reliés à la mondialisation et à la globalisation des pratiques d'affaires (Lundvall 2004 : 536).

Il apparaît donc que le recours à la notion de système national d'innovation enrichit la compréhension des dynamiques économiques et sociales de développement des territoires. II apparaît aussi que les principales contributions qui ont inspiré la structuration de cette notion misaient davantage sur la dimension spatiale des activités ; la localisation des institutions et des pratiques de toute sorte est donc au centre de l'intérêt de la littérature spécialisée. La dimension temporelle joue aussi un rôle important dans le développement des dynamiques d'un système national d'innovation. C'est que l'enchevêtrement des composantes de ce système, de même que leur développement, n'échappe pas à l'héritage d'un passé qui intervient, directement ou non, dans les processus de prise de décision. 
Comme l'avait constaté Simmie, la plupart des principes de base qui ont servi à formuler la notion de système national d'imnovation ont aussi servi pour établir le bien-fondé des systèmes régionaux d'innovation (Simmie 1997: 67). La section suivante porte sur ces systèmes.

\subsubsection{Le système régional d'innovation}

Depuis quelques années déjà, certains chercheurs enrichissent leur réflexion par le recours à la notion de système régional d'innovation. Cette expression témoigne de l'adoption de l'échelle régionale comme cadre général, que l'on considère appropriée à une meilleure compréhension des dynamiques économiques et sociales de développement d'un territoire. Le recours à cette notion a attiré à nouveau l'attention sur l'échelle spatiale qui serait la plus pertinente pour saisir les facteurs de localisation et de développement de l'activité économique et sociale.

Dans plusieurs publications récentes, c'est à l'échelle d'une région qu'il a paru le plus productif d'étudier les composantes institutionnelles en place, afin d'en comprendre l'influence sur les interactions entre acteurs individuels et collectifs. La région a ainsi servi de cadre à l'étude du système d'innovation, de même qu'à d'autres notions apparentées. Isaksen (2001) a présenté, dans le tableau ci-dessous, une comparaison intéressante entre ces différentes notions.

Tableau 1: La région, cadre spatial pour l'étude de l'innovation

\begin{tabular}{|l|c|}
\hline \multicolumn{1}{|c|}{ Concepts } & \begin{tabular}{c} 
Definitions and differences \\
\hline Regional cluster
\end{tabular} \\
\hline Regional innovation network & $\begin{array}{r}\text { concentration of 'interdependent" firns with the same or adjacent industrial } \\
\text { sectors in a small geographic area; } \\
\text { by trust, norms and conventions; }\end{array}$ \\
\hline Regional innovation system & $\begin{array}{r}\text { Co-operation between firms and different organizations for knowledge } \\
\text { development and diffusion; }\end{array}$ \\
\hline Leaming regions & $\begin{array}{c}\text { Increasingly organised co-operation with a broader set of civil organizations } \\
\text { and public authorities that are embedded in social and regional } \\
\text { structures. }\end{array}$ \\
\hline
\end{tabular}

Source: Isaksen, A. (2001), «Building Regional Innovation Systems: is Endogenous Industrial Development Possible in the Global Economy?», Revue canadienne des sciences régionales, XXV, 1, p. 104. 
Le tableau aide à bien distinguer les éléments d'un système régional d'innovation, qui sont essentiellement: «i) firms of a region's main industrial clusters, including their support industries; ii) supporting knowledge organizations, and iii) interaction between these actors» (Isaksen 2001: 107). Comme le constate l'auteur lui-même, cette perception correspond à celle définie auparavant par Cooke, Bpekholt et Todtling (2000), qui mettent l'accent sur «i) the knowledge application and exploitation sub-system, principally occupied by firms with vertical supply-chain networks; and ii) the knowledge generation and diffusion subsystem, consisting mainly of public organizations» (Isaksen 2001: 107). Cet aperçu de la pensée de quelques auteurs montre qu'un système régional d'innovation se distingue par l'intensité de pratiques de mise en commun des forces et des efforts des acteurs régionaux, afin de mieux faire face aux aléas des conjonctures. La coopération et la coordination entre ces acteurs, qui peuvent être privés ou publics, favorisent le développement des interactions et de l'innovation.

Par ailleurs, plusieurs écrits ont récemment tenté d'identifier les processus et les intercommunications fondamentaux qui régissent l'innovation au sein d'un système régional. Concernant la pertinence de l'échelle régionale, pour qui veut comprendre les dynamiques économiques et sociales de développement, Braczyk et al. (1998) insistent, parmi tant d'autres idées, sur l'importance de l'histoire, des routines, des établissements, de la différentiation et de l'incertitude. Ces éléments sont en quelque sorte responsables de l'orientation de la trajectoire régionale. Isaksen (2001) considère que l'adoption de l'échelle régionale facilite la tâche à qui veut comprendre les mécanismes de développement d'un territoire. Dans le tableau suivant, il a résumé certains problèmes d'ordre général, qui peuvent refréner les dynamiques économiques et sociales de développement d'une région.

Tableau 2: Obstacles au développement dans divers types de système réglonal d'innovation

\begin{tabular}{|c|c|c|c|}
\hline $\begin{array}{l}\text { Regional innovation } \\
\text { system problems }\end{array}$ & Type of problem & Typical problem region & Possible policy tools \\
\hline Organizational thinness" & Lack of relevant local actors & Peripheral areas & $\begin{array}{l}\text { Link firms to extemal } \\
\text { recourses + acquisition }\end{array}$ \\
\hline Fragmentation & $\begin{array}{l}\text { Lack of regional co- } \\
\text { operation and mutual trust }\end{array}$ & Some regional clusters & $\begin{array}{l}\text { Develop regional 'club goods' } \\
\text { and stimulate collaborative } \\
\text { efforts }\end{array}$ \\
\hline Lock-in & $\begin{array}{l}\text { Regional } \\
\text { specialized in outdated } \\
\text { technologies }\end{array}$ & $\begin{array}{l}\text { Old industrial regions } \\
\text { and raw-material-based } \\
\text { peripheral areas }\end{array}$ & $\begin{array}{l}\text { Open up networks towards } \\
\text { external actors }+ \text { local } \\
\text { mobilization }\end{array}$ \\
\hline
\end{tabular}

Source: Isaksen, A. (2001), "Building Regional Innovation Systems: is Endogenous Industrial Development Possible in the Global Economy? "Revue canadienne des sciences régionales, XXV, 1, p. 109. 
En général, on peut dire que les approches qui utilisent la notion de système régional d'innovation ont une perception originale des divers processus d'innovation. Selon les tenants de cette perception, l'innovation est un processus complexe, qui résulte d'un ensemble d'interactions entre les acteurs et organisations d'une région. De ce point de vue, tout processus d'innovation implique nécessairement des utilisateurs, des producteurs, des organisations et institutions, privées et publiques, et un ensemble d'intermédiaires qui font surtouł circuler, échanger les connaissances et le savoir. L'innovation peut donc être «characterized as a knowledge transfer and realization process involving actors whether internal or external to the specific firm operation as a project-based team or project-network» (Asheim et Cooke 1999: 157-158).

On peut aussi avancer que l'espace et les ressources d'une région deviennent cruciales pour la régulation des économies régionales. Le système régional d'innovation s'affirme done comme un niveau intermédiaire, entre le système national et les systèmes locaux. Cette situation fait du système régional un cadre idéal, propre à promouvoir la cohérence d'action devant s'accorder avec les négociations volontaires spécifiques intervenues entre les diverses composantes sociales (Ratti et al. 1997 : 37). C'est pourquoi un système régional d'innovation semble convenir davantage à qui veut analyser les relations étroites qui se développent entre l'espace, le savoir, les politiques locales, les systèmes industriels de production, l'information, l'apprentissage, les structures de gouvernance, les réseaux, les alliances et l'innovation (Ratti et. 1997). Cela rejoint l'hypothèse voulant que les approches, en termes de système régional d'innovation, doivent tenir compte des divers processus existants et de l'aptitude des acteurs régionaux à promouvoir la croissance économique (Acs 2000).

Les qualités conceptuelles d'un système régional d'innovation en font, aux yeux de plusieurs chercheurs, l'outil le plus approprié pour l'étude de la répartition spatiale, de l'utilité et de la qualité de l'activité économique. Toutefois, devant l'actuelle complexification des phénomènes, tant économiques que sociaux, une nouvelle génération de chercheurs ne parait pas entièrement satisfaite des systèmes régional et national d'innovation comme modèle explicatif, et propose un modèle aux contours modulables, plus exactement applicables à des situations diversifiées. 


\subsubsection{Le système spatial d'innovation}

Bien que l'on reconnaisse l'utilité théorique et pratique des systèmes régional et national d'innovation, certains auteurs doutent de leur capacité à rendre adéquatement compte des dynamiques actuelles. Oinas et Malecki sont de ceux-là. Ils avaient d'abord adopté le système régional d'innovation comme concept orienteur de leur réflexion, mais le trouvèrent incapable d'interpréter certaines réalités. Ils ont donc cherché un modèle plus apte à décrire des situations nouvelles résultant des récents changements technologiques, et ont formulé la notion de système spatial d'innovation.

L'utilisation de cette nouvelle expression illustre une double préoccupation. D'une part, ce concept se veut un outil théorique, propre à l'étude d'une réalité économique et sociale que l'appropriation des technologies d'information rend plus complexe, plus diversifiée. D'autre part les individus, comme les organisations qui s'approprient ces technologies, s'insèrent dans un ensemble indéfini de réseaux, chacun fonctionnant selon ses propres normes et selon des objectifs précis, inscrits dans le contexte socioéconomique en place.

Le concept de système spatial d'innovation permet d'inscrire les interactions économiques et sociales dans un cadre spatial approprié, de reconnaître les enjeux concrets d'espaces et de territoires avantagés par un haut degré d'appropriation des TIC. Ces enjeux, auxquels correspondrait un niveau particulier d'innovation, varient constamment en fonction des stratégies qu'adoptent les acteurs pour répartir leurs activités dans l'espace qu'ils occupent. Une meilleure compréhension de l'ampleur de cette notion, d'emblée théorique, suppose que les interactions entre les acteurs localisés dans un même espace constituent un moyen approprié pour reconnâ̂tre l'innovation (Malecki et Oinas 1999 et Oinas et Malecki 2002: 103).

Le système spatial d'innovation réfère à la capacité d'une collectivité, implantée dans un espace donné, à établir des liens avec un ensemble de réseaux, soit locaux, régionaux, nationaux ou internationaux. I1 repose aussi sur la récognition de la compétitivité accrue des acteurs, qui savent profiter d'une appropriation convenable des nouvelles technologies, particulièrement des TIC, appliquées ensuite à des stratégies d'apprentissage propices au développement d'un territoire. 
En somme, on peut concevoir le système spatial d'innovation comme une approche qui associe les principales caractéristiques des systèmes régional et national d'innovation, sans pour autant se confondre avec ces deux vocables. Il les dépasse, car un rôle important est donné aux nouvelles technologies et au réseautage, à l'origine de nouvelles formes de proximité et de la compression de la distance.

\subsection{Les TIC diversifient les horizons}

Il y a peu d'années, un territoire peu doté en ressources naturelles ou désavantagé par la géographie, ne pouvait guère espérer une croissance satisfaisante de son économie et progresser en même temps sur les autres plans, au rythme de territoires mieux nantis ou fortement urbanisés. Il apparaît pourtant que cette situation n'est pas inaltérable; que dans les territoires périphériques comme dans les grandes villes, l'innovation et le progrès qu'elle entraîne ne sont pas fatalement hors d'atteinte. Nous avons vu que les collaborateurs du GREMI ont élaboré des outils théoriques qu'ils se sont efforcés d'adapter aux exigences des conjonctures. De plus l'avènement des TIC a grandement changé la donne et offre une possibilité unique d'envisager un meilleur équilibre des chances. Où que l'on regarde, l'appropriation des TIC constitue désormais un facteur essentiel d'avancement technique et devrait faciliter la dépolarisation du développement.

Des chercheurs provenant de divers horizons se sont intéressés aux effets des TIC sur les dynamiques économiques et sociales de développement. Ils ont abouti à des conclusions divergentes qui attisent l'intérêt pour des recherches ultérieures. On s'entent actuellement pour dire que la conjoncture actuelle favorise le déplacement des axes de la concurrence, laquelle se situe désormais non pas seulement entre entreprises mais entre pays. Shearmur écrit :

Nous assistons au jeu de la concurrence entre pays, où chaque pays tend à se spécialiser dans les fonctions économiques pour lesquelles il est le plus adapté. Même si on peut se questionner sur le bien-fondé des pratiques de mondialisation actuelles - qui semblent parfois aller à l'encontre d'une ouverture réelle des marchés - nous ne devrions pas être angoissés lorsqu'on observe certaines industries et fonctions économiques pour lesquelles les marchés s'ouvrent réellement!» Shearmur (2004a). 
La concurrence demeure néanmoins vive entre les entreprises. Harrison et al. (1996) ont analysé les comportements des entreprises innovatrices dans leurs relations avec leurs milieux en fonction des changements technologiques. Ils se sont intéressés aux entreprises, plutôt qu'aux dynamiques locales. Analysant la localisation et le développement des entreprises, l'objectif était de comprendre les mécanismes qui influencent la distribution spatiale des innovations. A partir d'un échantillon aléatoire de 1000 entreprises, l'ouvrage permet de constater que la probabilité que les dirigeants adoptent une nouvelle technologie est significativement associée au degré d'urbanisation des comtés dans lesquels elles sont implantées. Il y est démontré aussi que l'innovation d'une entreprise dépend principalement de sa taille, de la diversification de la production, de l'intensité des relations sociales en place, de l'interaction de l'industrie avec certains secteurs de l'administration publique, etc. Les auteurs ajoutent :

with respect to the contrast between diverse locales versus clusters of similar economic activities - a contrast that has informed applied economic and geographic research since the 1930s - we find strong support for the importance of diversity in the local socioeconomy for promoting innovative firm behavior. By contrast, once the technical and organizational properties of the establishments themselves are fully taken into account, we find that sectoral specialization (or, to use the classical Marshallian term, localization) has almost no explanatory power in shaping the likelihood that metalworking plants will have adopted one or more PA tools (Harrison et al. 1996: 251-252).

De leur côté, Blundell et al. (1999) ont cherché à comprendre les relations qui existent entre l'innovation, les choix technologiques des entreprises et leur part de marché. Leur analyse a permis de dégager des corrélations entre la capacité d'innovation d'une entreprise, sa force concurrentielle et sa part de marché. Ils ont aussi démontré que l'innovation a des effets plus marqués quand il s'agit de sociétés ayant un poids important dans leur marché (Blundell et al. 1999: 550). Licht et Moch (1999) se sont intéressés aux effets des TIC sur les dynamiques de divers milieux. Ils ont analysé les pratiques des entreprises qui oeuvrent dans le secteur des services en Allemagne et ont démontré que le recours à des composantes technologiques, comme les ordinateurs, accompagné d'un investissement raisonnable en capital d'équipement, de $R \& D$ et de capital humain, a une influence positive sur la prospérité des affaires et donc de leur capacité concurrentielle. Selon ces auteurs, l'appropriation de la technologie influe sur presque toutes les activités d'une entreprise, mais certains dirigeants sont moins sûrs: 
«although a high percentage of innovating firms claims to have realized productivity gains, managers of service firms seem to be less convinced about the productivity benefits of IT investments» (Licht et Moch 1999 : 378).

Dans le contexte canadien et québécois, Polèse et Shearmur (2002) ont récemment publié une étude remarquée, où ils ont tenté de saisir le rôle des NTI dans le développement de régions périphériques du Canada (Québec et provinces atlantiques). Les auteurs ont voulu voir comment les technologies d'information y contribuent à une nouvelle dynamique économique. Ils concluent que les NTI vont accentuer la concentration des activités économiques dans les grandes villes. Bien qu'elles ajoutent de la valeur aux activités économiques, les NTI accentueront les tendances observables dans nos économies: elles continueront à favoriser une répartition inégale dans l'espace de l'activité de production. Les auteurs écrivent :

l'importance désormais accordée à la valeur ajoutée et au savoir ne simplifiera pas la vie aux entreprises des régions périphériques. Il n'y a aucune raison de croire que cette évolution va accroitre l'avantage comparatif de ces communautés pour ce qui est de la localisation des activités manufacturières et des services marchands. Bien au contraire, tout indique que l'avantage comparatif va continuer, à tout le moins pour la fabrication de la plupart des produits à valeur ajoutée élevée, de se déplacer vers les grandes villes et les autres localisations centrales, qui se retrouveront ainsi encore plus favorisées (Polèse et Shearmur 2002:62).

Le développement d'une économie du savoir ne changerait donc pas le sort des entreprises implantées dans un territoire périphérique. Les tendances actuelles se maintiendront et le développement inégal des territoires persistera:

l'avènement de l'économie du savoir et des nouvelles technologies de l'information ne devrait pas modifier sensiblement les tendances actuelles de la répartition spatiale de la population et de l'emploi. Dans l'ensemble, son impact sera d'accentuer ou de renforcer ces tendances. La propension des activités riches en savoir à se localiser dans les grands centres urbains n'a pas changé. La concentration à long terme de l'emploi et de la population à l'intérieur et dans le voisinage des régions urbaines importantes se poursuivra (Polèse et Shearmur 2002:61-62). 
Les auteurs montrent que les NTI encouragent la concentration des activités économiques. La géographie imposera certaines limites car l'éloignement de nombre de territoires freinera l'émergence de véritables dynamiques de développement. C'esţ dire que les NTI n'influencent pas les déterminants fondamentaux de la localisation de l'activité économique. De ce fait,

la distance n'est pas abolie et n'est pas près de l'être. L'éloignement des grands centres urbains continuera d'entraver le développement économique des régions périphériques. Les déterminants fondamentaux de la localisation des industries ont très peu changé depuis quelques décennies. Les biens et les personnes doivent encore et toujours se déplacer (Polèse et Shearmur 2002:62).

L'usage des NTI a néanmoins des conséquences majeures. Cet usage influence la vie sociale puisqu'on se sert des NTI pour répondre à des besoins variés et immédiats. De même, cet usage agit sur le cours des activités économiques: les NTI s'intègrent à un grand nombre d'outils et d'objets utilisés dans les secteurs de ces activités. Elles améliorent sensiblement les activités de production, de commercialisation et de traitement de l'information. De ces points de vue, les NTI ont des effets positifs dans certains territoires :

les NTI ont quand même des effets positifs pour les régions périphériques. En facilitant l'accès des firmes locales à l'information, elles améliorent la productivité et favorisent l'innovation. Dans la plupart des communautés éloignées, le manque d'accès à l'information a sans doute cessé de constituer un grave obstacle à l'entrepreneurship et à l'innovation. Pour les entreprises qui exportent hors de leur région, les NTI sont un moyen de mettre leurs produits en marché de façon plus efficace et de rester proches de leurs clients (Polèse et Shearmur $2002: 62$ ).

Les NTI ont un potentiel qui déterminera le sort réservé à certains territoires. En plus de modifier plusieurs pratiques sociales et économiques, elles susciteront des dynamiques nouvelles :

dans les années qui viennent, les impacts positifs les plus importants des NTI vont sans doute s'exercer dans le secteur public, notamment dans la santé et l'éducation [...]. Les NTI vont néanmoins, on peut s'y attendre, créer de nouvelles possibilités pour mieux desservir les communautés périphériques, permettant de leur assurer plus efficacement des niveaux acceptables de bien-être [...] Certes, il ne faut pas croire que la prestation électronique de certains services 
renversera les tendances actuelles. Mais il n'est pas complètement irréaliste de penser, par exemple, que l'enseignement poste-secondaire à distance atténuera les pressions qui amènent les jeunes à émigrer pour continuer leurs études .(Polèse et Shearmur 2002:61).

Ces conclusions, on l'aura noté, concernent toute la moitié orientale du Canada. C'est dans le cadre de cet ensemble géographique qu'il faut situer ces vues sur l'apport des NTI à la dynamique économique de développement des territoires périphériques. La réflexion des auteurs a principalement porté sur les nouvelles technologies de l'information. Or ces technologies incluent également un autre aspect, qui paraît tout aussi important: la communication. Si on avait examiné la communication avec autant d'attention que l'information, il serait intéressant de voir si les conclusions seraient demeurées les mêmes. C'est en effet la dimension communication, avec l'information, qui distingue les TIC des techniques traditionnelles. C'est elle qui supporte le développement de la société en réseau et la mise en place du "village global".

À l'échelle de l'Histoire, la technologie de l'information et de la communication, telle que rendue possible par l'informatique, demeure encore un phénomène tout récent, qui date d'à peine une génération. Les conséquences à long terme de cette avancée sur l'activité économique, le développement régional et le devenir social demeurent encore imprécises et feront nécessairement l'objet de futures études. Pour le moment, il semble qu'on devrait plutôt attendre des retombées positives. L'invention du chemin de fer, pour ne citer qu'un exemple, a certes bénéficié aux villes, mais aussi a permis une meilleure occupation du territoire, a accéléré le développement des campagnes, favorisé la décentralisation de l'activité économique et de l'administration. Est-il utopique de penser que les retombées des TIC peuvent avoir des effets similaires pour le développement de nos régions?

\subsection{De nouvelles donnes conjoncturelles : la proximité et la distance}

L'étude du développement, que ce soit sous l'angle de milieu innovateur, ou des systèmes régional, national, spatial d'innovation, laisse un pan important de la réalité à expliquer. Comment les forces économiques et sociales sont-elles conditionnées par des facteurs liés à la géographie et à la localisation des activités ? Si l'on considère que le cadre spatial, le territoire, est le substrat de l'activité économique et de la vie sociale, deux questions 
d'importance se présentent: 1 . Comment ce substrat, qui tient de l'histoire mais surtout de la géographie, agit-il sur les pratiques économiques et sociales? 2. Comment l'appropriation croissante des TIC peut-elle modifier l'influence de ce substrat? Ces questions sont assurément en relations avec les notions de proximité et de distance, et de leur rôle dans l'économie contemporaine.

\subsubsection{La proximité, une notion au contour extensible}

La notion de proximité tend à s'élargir. Bien que le terme soit d'ordinaire lié à la géographie, les économistes, les sociologues, les historiens et d'autres spécialistes n'hésitent guère à l'associer à des phénomènes nouveaux et à des pratiques émergentes. La littérature qui traite de milieu innovateur est naturellement appelée à parler de proximité, et les auteurs en décrivent les deux principales formes : géographique et organisationnelle.

Dans son sens premier, la proximité renferme l'idée de voisinage, de distance courte dans le temps et l'espace. Le voisinage amène généralement à se mieux connaitre et, au besoin, à travailler de concert. C'est pourquoi la proximité géographique incite les entreprises d'un même milieu à se concerter, à coopérer et à coordonner leurs actions et stratégies. Ces entreprises subissent en effet les mêmes contraintes, et elles ont plus de chance d'y résister si elles mettent en commun leurs forces. La proximité géographique peut devenir plus ou moins importante, selon les caractéristiques du découpage territorial, par la disponibilité ou non d'infrastructures telles, par exemple, que des moyens de transport adéquats; elle peut avoir un rôle structurant et mobilisateur qui donne sens aux efforts individuels et collectifs, d'autant mieux que règne un large consensus. La proximité appelle des distances courtes, des frontières rapprochées, «des relations privilégiées entre différents acteurs, des relations dont une partie est physiquement proche» (Julien 1996 : 97).

L'approche industrielle met l'accent davantage sur la proximité organisationnelle que sur la proximité géographique; elle affirme que les processus d'innovation technologique se développent selon une logique évolutionniste propre à l'économie industrielle. Les travaux de Ratti et al. (1997), importants pour comprendre l'utilité des «dynamiques de proximité ", illustrent l'utilité de ces perceptions. 
La proximité est organisationnelle en ce qu'elle traduit la séparation économique entre acteurs, groupements structurés et institutionnels. On y trouve l'idée d'ordre, de méthodes, de principes que des citoyens regroupés se donnent librement. Salais et Pecqueur mettent en rapport la proximité géographique et la proximité organisationnelle de la façon suivante :

la première (géographique) ne se confond pas avec la proximité physique dans la mesure où elle n'est pas donnée par les contraintes naturelles, mais elle est construite socialement. La seconde (organisationnelle) dépend principalement des représentations en fonction desquelles les agents inscrivent leurs pratiques (stratégies, décisions, choix, etc.). Elle concerne les relations interindividuelles, mais surtout la dimension collective, à lintérieur des organisations ou entre les organisations; la proximite territoriale serait l'intersection des deux formes de proximité (Salais et Pecqueur $1996: 216$ ).

Une autre forme de proximité s'établit lorsque les entreprises d'un territoire tendent à s'intégrer aux réseaux locaux d'innovation, et qu'elles cherchent collectivement à s'impliquer dans les pratiques d'autres composantes du milieu, des pratiques qui favorisent à la fois le développement de ces réseaux et le renouvellement de leurs stratégies.

Une littérature importante est consacrée par ailleurs à l'économie de proximité. Située à la croisée des chemins entre l'économie spatiale et l'économie industrielle, l'économie de proximité réfère à l'analyse des facteurs propres à un espace donné, des facteurs qui jouent un rôle important dans la coordination des activités économiques. Selon ces vues, l'espace n'est plus un élément neutre, mais plutôt une composante principale de l'activité économique. Il s'agit généralement d'un espace économique, où la proximité prend un aspect économique lorsque les interactions entre les entreprises d'un territoire deviennent plus décisives. La. proximité économique entre deux entreprises peut être estimée par la durée et l'intensité de leurs échanges de produits (Lundvall 1992a : 370). Selon Lundvall, la proximité varie selon que les acteurs sont endogènes ou exogènes à un territoire ; l'exogénéité des individus fait que ces acteurs tardent à développer une confiance mutuelle. Dans d'autres cas, plus les individus développent des comportements loyaux, plus la probabilité du caractère coopératif et la confiance sont fortes. II s'ensuit que si des entreprises coopèrent entre elles, on peut penser que la confiance est d'autant plus forte que les relations ont été durables et intenses. Cela incite l'auteur à faire valoir qu'une proximité relationnelle plus forte se développe entre les acteurs dès que s'établissent des sentiments mutuels d'identification et de loyauté. 
La proximité économique peut être considérée comme une variante de la proximité organisationnelle. Elle est une relation unique qui s'établit entre des entreprises, des organisations, une relation fondée essentiellement sur la confiance qui s'est construite au fil du temps par l'échange d'information, de biens et de services. Cette proximité peut exister malgré la distance, mais elle est naturellement plus agissante quand des acteurs, situés dans un même espace géographique, peuvent régler leur position stratégique selon leur aptitude à bien intégrer les dynamiques locales.

Avec le temps, les relations qui s'établissent entre les entreprises et entre les employés tendent à former des réseaux qui peuvent être davantage influencés par l'existence de facteurs institutionnels, que par la distance géographique qui les sépare (Lundvall 1988). Ainsi s'établit une proximité particulière par le développement de réseaux entre les utilisateurs et les producteurs (Lundvall 1992). Avantageuse pour tous les intéressés, cette proximité repose sur des liens intangibles que chacun a intérêt à cultiver.

Par ailleurs, «la proximité des lieux de production ne simplifie pas seulement la charge des coûts de transport mais aussi facilite la circulation de 1'information (...) La proximité de nombreuses entreprises favorise l'innovation» (Ratti $1992: 58-59$ ). Toutefois la proximité, de quelque nature qu'elle soit, n'est pas acquise partout : il faut la stimuler, la manifester et l'exploiter pour ajouter de la valeur aux initiatives de développement. Cela se réalise essentiellement par la promotion, dans un territoire, du potentiel collectif dont dispose un groupe d'individus ou d'acteurs. Alors la proximité prend forme de

synergies et d'économies externes : celles-ci seraient déterminées soit par des relations tout à fait informelles dues à l'identité, à la proximité et à un processus de création de savoir-faire spontané, soit à des formes territorialisées de stratégie, formalisées et relevant de la nature organisationnelle du réseau (Ratti $1992: 59$ ).

Dans le cas de l'économie de l'information, la proximité revêt une importance particulière. Plusieurs études ont scruté les dimensions géographiques de l'information et ses implications sur la distance qui sépare les producteurs et les utilisateurs. Dans l'économie d'information, la proximité géographique entre les producteurs et les utilisateurs n'a que des effets très limités sur les processus d'innovation (Cornish $1997: 162$ ). Cet auteur s'est intéressé aux origines géographiques de diverses composantes de l'information et aux implications de la distance 
entre les producteurs de cette information et ses utilisateurs. Cette étude lui a été utile pour analyser le fonctionnement d'un marché de l'intelligence, et les résultats montrent que les acteurs oeuvrant dans ce marché le font dans les activités qui incitent à l'innovation (Cornish 1997 :162). Selon l'auteur, dans le "marché de l'intelligence", la distance géographique importe peu.

Certes, l'avènement des TIC a ajouté une dimension nouvelle à la proximité: c'est «l'existence d'un fantasmatique, d'un nouveau type de proximité, de téléproximité sociale, qui renouvelle complètement le voisinage, l'unité de temps et de lieu de la cohabitation physique) (Virolio 1999 : 37). Cette forme de proximité abolit les liens de contiguité entre les gens, les territoires, et via l'électronique en crée de nouveaux, virtuels, souvent anonymes, avec des interlocuteurs aux confins du monde. L'instantanéité et la facilité des communications autour de la planète constituent un événement majeur dont on ne peut présentement que supputer les conséquences à long terme.

Par ses dimensions cognitives, organisationnelles, sociales, institutionnelles et géographiques, la proximité influence l'innovation. Une meilleure combinaison de ces dimensions peut avoir des conséquences heureuses. En fait, chacune de ces dimensions influe sur l'apprentissage et sur l'innovation, stimulant ainsi la croissance économique (Boschma 2004).

La notion de proximité semble particulièrement pertinente pour rendre compte de la manière dont les acteurs s'adaptent aux contraintes que posent la distance, un cadre commun, la concurrence. Considérée sous ces aspects, Porter constate que la proximité physique «apporte des avantages pour la productivité et l'amélioration de la productivité (facilité d'accès, relations, informations, motivation, etc.) qu'il serait difficile d'obtenir à distance» (Porter 1999 : 246). De la proximité surgissent, en effet, des attitudes et des comportements, des alliances et des coalitions qui consolident (parfois sapent) les capacités concurrentielles des acteurs et des territoires.

Plus récemment, Rallet et Torre proposent une analyse intéressante : ils montrent que des liens forts peuvent exister entre les sentiments de proximité et la localisation des activités. Ils mettent l'accent sur les dimensions géographiques et organisées de la proximité (Rallet et Torre: 2004). Les auteurs mettent en garde contre la confusion souvent faite entre 
l'agglomération et les interactions de proximité géographique. Des interactions plus denses ne nécessitent pas uniquement des distances courtes, elles exigent obligatoirement des comportements et des pratiques qui émanent de l'encastrement social et institutionnel d'un ensemble de valeurs et de normes communes. Cette perception permet de voir que les besoins de proximité géographique (pour réaliser une coopération, par exemple) ne supposent pas nécessairement que les agents soient localisés dans un même espace géographique.

Cela veut dire que des rapports nouveaux, quant au lieu géographique, à l'espace géographique sont en train de prendre forme. Sous l'influence des TIC, et d'Internet plus particulièrement, la proximité doit être appréhendée en tenant compte de la "fragmentation" de l'espace géographique. Couclelis a eu recours à cette notion de fragmentation de l'espace pour rendre compte des transformations que les TIC provoquent dans le monde des affaires et dans la localisation des activités économiques en général (Couclelis 2004). Accompagnée d'un ensemble de nouveaux réseaux, cette fragmentation de l'espace remet en question les fondements de la prépondérance urbaine et conditionne le développement d'un autre ordre, encore en construction, qui serait plus favorable aux territoires éloignés des pôles économiques.

Pour Malecki, cependant, cette sorte de concaténation en devenir problématique. Où que nous soyons, selon lui, nous vivons à une époque marquée par la formation d'une variété de réseaux, où le choix des bonnes stratégies est crucial, il estime que pour survivre, l'entrepreneur doit baser sa stratégie sur l'innovation. Or on peut innover soit en exploitant davantage le potentiel des TIC, soit en profitant au maximum des activités qui ont traditionnellement constitué un avantage concurrentiel : «in both cases, however, we witness a reinforcement of urban function, and hence the conclusion is warranted that cities are the sites par excellence for entrepreneurial innovations in a modern network era» (Malecki et al. 2004: 2).

Pour la plupart des chercheurs, l'influence de la proximité et de ses formes diversifiées, ne fait guère de doute, mais ses manifestations continuent de stimuler la réflexion. Déjà modelée par une multitude de facteurs, la proximité a pris, avec l'avènement des TIC, une dimension nouvelle aux conséquences importantes mais encore imprécises. Elle est généralement vue 
comme un facteur incitant à l'interaction et à la coopération entre acteurs et, en dernière analyse, favorable à la croissance.

\subsubsection{La proximité et l'apprentissage}

Pour les acteurs rêvant de développement, d'association des forces de leur entourage, il devient vite apparent que la proximité, réelle ou virtuelle, offre des avantages qu'il y a lieu d'exploiter. La réflexion peut entrâner une évolution des perceptions et des pratiques, mais davantage, à prendre conscience d'une possibilité inappréciable offerte par la proximité : l'occasion d'apprendre collectivement. Plusieurs études ont déjà montré que la proximité favorise l'apprentissage et l'acquisition des connaissances désormais indispensables à une bonne gouvemance, donc à la réussite et à la prospérité d'une entreprise.

Selon Nooteboom (2000), il existe une relation importante entre l'apprentissage et les formes diverses de gouvernance et donc de proximité. Cette relation est conditionnée par la production et l'échange de la connaissance. Selon l'auteur, la distance peut être cognitive et agit alors sur la gouvernance, car elle encourage le transfert des connaissances par interaction. La différence entre la stabilité et le changement, dans les relations qui se développent autour de la production et de l'échange des connaissances, dépend des efforts fournis par chaque acteur concerné.

Pour Oinas (2000) les formes diverses de la proximité jouent un rôle important dans la circulation des connaissances, y compris la connaissance transférée par l'intermédiaire du mécanisme de marché. Cette circulation fait voir que ces diverses formes de proximité réunissent la proximité géographique, culturelle, et linguistique. De plus, selon Malecki et Gormain (2001), un ensemble de conditions liées à l'environnement local et au climat d'entrepreneuriat d'un milieu, de même qu'aux diverses formes de proximité, favorisent la synergie. Ces conditions agissent positivement sur l'activité économique dès lors qu'un milieu profite des réseaux locaux, de la disponibilité de capitaux à risque, de la présence d'établissements de support locaux.

Ces considérations ramènent ainsi à des notions qui se touchent : le territoire, le milieu, et à une autre facette de la proximité : la territorialité. La notion de territorialité a acquis, surtout 
avec l'avènement des TIC, un contenu élargi qui modifie graduellement les liens entre les acteurs de développement et leurs rapports aux composantes de leur territoire (Fortin et al. 2004). 11 reste cependant que, pour la plupart des hommes, un lieu d'ancrage est important, sinon nécessaire, et que le sentiment d'appartenance à ce lieu s'objective généralement en un sentiment puissant : la territorialité.

La territorialité réfère à l'appropriation des caractéristiques et des valeurs territoriales; elle est le prolongement d'une prise de conscience collective qui fait naitre la confiance et la solidarité des citoyens, en vue d'objectifs partagés. Bref, la territorialité est un élément irremplaçable de l'homogénéité sociale. Quand celle-ci change, celle-là aussi. Elle révèle «notre manière de percevoir et d'évaluer le contexte et dit le double sentiment d'une appartenance précise, et d'une domination vague sur l'espace» (Piveteau 1995: 161).

La territorialité représente ce par quoi les acteurs s'identifient, à un moment précis, à la fois à un territoire et aux rapports à celui-ci. Elle exprime le désir, mais aussi la nécessité, qu'éprouvent les individus de contrôler le jeu des rapports des composantes spatiales et temporelles qui les concernent. La possibilité offerte dorénavant par l'Internet de créer une proximité virtuelle renforce le sentiment de territorialité et encourage une évolution attentive à intégrer les acteurs et les groupes qu'ils forment au sein des tissus sociaux existants (Fortin et al. 2004). Toutefois, malgré l'instabilité apparente de la territorialité et du sentiment d'appartenance, il demeure, chez les individus et les groupes sociaux, une volonté invétérée d'inscrire leurs rapports, internes ou externes, dans un cadre qui leur soit familier.

Au terme de ce chapitre, il paraît fondé d'avancer que les divers concepts utilisés jusqu'à présent pour appréhender des réalités économiques et sociales multiformes ont perdu de leur pertinence; ils ne peuvent en effet que rendre compte imparfaitement des dynamiques économiques et sociales qui démarquent le développement de certains territoires périphériques. Les changements technologiques et l'évolution conséquente de la société sont tels que des concepts somme toute récents: les systèmes local, régional ou national d'innovation, ne paraissent plus en mesure de décrire adéquatement la réalité actuelle. Avec leur système spatial d'innovation, Oinas et Malecki ont tenté de dépasser les insuffisances associées au recours à ces concepts. Il semble que cette notion permet de montrer qu'un changement technologique ne survient pas nécessairement dans un cadre spatial et temporel 
bien précis. Et que le recours à cette notion permet de dépasser les limites des systèmes antérieurs et d'expliquer des réalités complexes. Bien que ces chercheurs aient jusqu'à présent peu développé leur concept, leur initiative est de grande utilité, scientifique et pratique, néanmoins des efforts restent à faire dans cette direction.

\section{Conclusion}

Les conditions existantes dans un milieu, un territoire, influent sur la genèse de l'innovation. Il se dégage de la littérature consultée que des liens forts existent entre l'appropriation des technologies, particulièrement des TIC, et le dynamisme économique et social. Ces technologies activent les éléments déterminants dans un territoire ou un milieu, en favorisant la proximité, la concertation et de nouvelles manières d'apprendre; elles contribuent à diversifier les horizons qui s'ouvrent devant des acteurs mieux préparés à évaluer une conjoncture.

Pour apprécier l'aptitude d'un territoire ou d'un milieu à innover, on est amené à considérer l'usage et les effets des technologies. À notre époque, il semble que c'est d'abord l'usage judicieux des TIC qui y stimule le plus le dynamisme. Toutefois quand un développement technologique intervient quelque part, il s'inscrit dans un contexte historique et social particulier, dans un cadre spatial défini; les conditions et le parcours historique de cet espace influent singulièrement sur la marche de ce développement et font que le parcours technologique soit en quelque sorte le reflet des rapports entretenus entre ce milieu et la technologie.

Pour mener à bien l'étude de ces questions, le choix d'un cadre spatial est nécessaire, et en même temps problématique. Quel échelon territorial faut-il privilégier? Selon les chercheurs, le choix a porté sur l'échelon national, régional ou local, chaque fois avec des conclusions intéressantes. Ils ont noté d'abord que le cadre institutionnel en place conditionne le déroulement des activités économiques et sociales; que ce cadre fournit un contexte où les acteurs profitent des flux d'information, des biens et des services nécessaires au succès de leurs activités. Par ailleurs les activités économiques, l'innovation, sont inégalement réparties dans un territoire et, chez les acteurs, l'usage restreint de l'information et de son économie, contribue à cette inégalité. L'abondance ou la pauvreté des ressources dont disposent les 
territoires sont évidemment sources d'inégalité, de même que les comportements variés des acteurs.

C'est à la lumière de ces idées qu'il convient d'examiner le processus d'implantation d'une technologie et ses effets dans un territoire. Pour la clarté de la suite de ce travail, il parait utile d'en revoir brièvement le canevas, articulé autour du changement technologique et de l'aptitude des territoires et des acteurs de développement, à créer d'abord les conditions nécessaires à l'innovation, et ensuite, à savoir en profiter.

Afin de comprendre comment l'appropriation des technologies influence le développement des territoires, des sujets tels que l'informatique, le changement technologique et les TIC ont été discutés. Il a été montré qu'un changement technologique favorise linnovation, que l'introduction de l'informatique et l'usage de l'Internet ont des effets observables dans tous les territoires, ouvrant du même coup des horizons nouveaux devant les acteurs. Ces effets influencent non seulement le déroulement des activités d'un acteur mais peuvent, la conjoncture aidant, avoir une influence décisive sur sa localisation et sur la nature des rapports qu'il entretient avec son environnement proche ou éloigné.

Nous nous sommes de plus intéressé aux stratégies adoptées par les acteurs qui réalisent limportance et l'utilité d'une technologie d'avant-garde, et nous en avons tiré des enseignements sur l'importance de l'information stratégique et du réseautage. Et il s'est avéré que, lorsque appréhendées dans le cadre d'une conjoncture donnée, la richesse de l'information, la complexité de son économie, la diversité des réseaux et des intérêts qu'ils défendent, sont autant d'éléments favorables à l'innovation. En somme, pour les acteurs de développement qui savent exploiter convenablement le dynamisme d'une économie d'information et qui s'affilient à des réseaux d'entreprises, la probabilité d'innover est plus grande. Si, comme il appert, l'innovation procède de l'initiative des acteurs et de l'aptitude des économies à intégrer l'ordre informationnel, il semble que la mise en place de stratégies d'apprentissage individuel et collectif doive être vue comme une condition essentielle à la prospérité des affaires et au développement.

Il a paru tout aussi utile de scruter les effets de la taille des entreprises. La littérature contemporaine indique qu'actuellement linnovation est généralement orientée par l'aptitude 
des acteurs à prendre conscience de la nécessité de s'adapter aux vagues successives provoquées par l'internationalisation des enjeux économiques et socioculturels et par la globalisation des marchés. Il ne fait guère de doute que l'appropriation accrue des technologies et l'explosion de l'Internet dans le monde des affaires constituent un avantage pour les acteurs et les territoires qui savent les exploiter. La nature des dynamiques propres à léconomie d'information tend à dissocier linnovation de la stature économique de l'acteur. C'est pourquoi la taille de l'entreprise n'a plus guère d'importance. Il semble toutefois que la vitalité d'une région donnée dépende de certains facteurs qui paraissent agir plus ou moins à l'insu des acteurs. Nous avons donc tenté de cerner ces facteurs en nous intéressant aux dynamiques économiques et sociales des milieux et des territoires.

Bref, le développement et l'innovation dans un milieu ou dans un territoire dépendent largement de facteurs endogènes. Les composantes d'un territoire participent activement à développer des formes nouvelles de solidarité, de coopération et de sentiments d'appartenance. Lorsque les TIC y sont appropriées convenablement, elles encouragent l'apprentissage individuel et collectif et y conditionnent le changement technologique. 


\section{CHAPITRE 5}

\section{TERRITOIRES ÉTUDIÉS ET OFFRE DiNFORMATION}

De la revue de littérature qui précède ressortent des expériences diversifiées et des notions utiles à la formulation du cadre théorique et conceptuel de notre recherche. La première section de ce chapitre présente les territoires retenus pour cette étude, les compare et en fait voir les forces et les fragilités par rapport aux structures économiques et sociales. La seconde section examine l'offre d'information émanant de sources variées, en vue de saisir comment cette information contribue à enrichir la dynamique de ces territoires.

\subsection{Les territoires étudiés}

Cette section présente les territoires objets de cette étude et en montre les principales caractéristiques économiques et sociales.

\subsubsection{Présentation générale}

Le territoire du Québec est divisé en 17 régions administratives, divisées à leur tour en 97 municipalités régionales de comté, les MRC. Une MRC est une institution supramunicipale. Elle regroupe l'ensemble des municipalités urbaines et rurales d'une même région 
d'appartenance, à l'exception des municipalités qui font partie des communautés urbaines de Montréal, de Québec, de l'Outaouais, de la municipalité de la Baie-James et de la municipalité de Côte-Nord-du-Golfe-du-Saint-Laurent.

Pour les fins de notre analyse, nous avons retenu trois MRC : du Saguenay, de Manicouagan, de Rivière-du-Loup, et la communauté urbaine de Québec (CUQ). Ces territoires sont situés dans quatre régions administratives différentes, dont les ressources ainsi que le potentiel économique et social présentent une variation considérable.

Les MRC et les communautés urbaines furent créées afin de foumir aux territoires concernés un cadre global d'aménagement et d'urbanisme. 11 y avait en effet dans le vaste espace public un morcellement des objectifs et des responsabilités qui rendait nécessaire la mise en place d'un organisme capable de susciter et de coordonner le développement dans un territoire, et de l'articuler avec les stratégies de croissance adoptées par les divers paliers de gouvernement. Plusieurs mesures politiques ont encadré l'insertion des MRC au sein du système national et de fait, depuis leur création en 1979 , ces territoires n'ont pas manqué d'influer sur la dynamique de développement. On a dit avec justesse des MRC que "leur légitimité idéologique s'appuie sur le principe de territorialité ou d'appartenance territoriale", et que «le territoire d'appartenance MRC représente l'assise de solidarité supra-locale» (Proulx $1995: 142-143$ ).

Tableau 3: Les MRC étudiées : population et superficie

\begin{tabular}{|l|l|l|l|}
\hline Territoire MRC & Région & Population totale & Superficie (en Km2) \\
\hline Communauté urbaine de Québec & Capitale Nationale & 693790 & 3347,11 \\
\hline Saguenay & Saguenay-Lac-Saint-Jean & 158740 & 4396,71 \\
\hline Riviére-du-Loup & Bas-Saint-Laurent & 32110 & 1267,44 \\
\hline Manicouagan & Cóte-Nord & 32833 & 2029,15 \\
\hline
\end{tabular}


Plusieurs organisations, cuvrant dans plus de 25 secteurs d'activité, assurent la mise en valeur de chaque territoire MRC; Proulx a identifié ces 25 secteurs (Proulx 1998). Tenant un rôle intermédiaire entre les paliers local et national, ces territoires ont su en général tirer avantage des politiques et mesures gouvemementales, et ont exercé une gestion satisfaisante. Proulx constate

qu'après seulement dix ans d'existence, de nombreuses fonctions publiques utilisent les territoires MRC comme aire de gestion. Ce progrès rapide dans la construction institutionnelle MRC démontre bien la pertinence de cet échelon territorial dans la gestion publique au Québec (Proulx 1995 : 38).

En fait, les initiatives locales et régionales trouvent dans chaque territoire MRC une plateforme qui veille à l'harmonie de la dynamique économique et sociale de développement. Les territoires $\mathrm{MRC}$ constituent des réalités homogènes, où les sentiments d'appartenance sont très forts, d'autant plus que le découpage de ces territoires a tenu compte des caractéristiques physiques et humaines.

Chacun des quatre territoires a un parcours historique qui lui assigne une place particulière au sein du système national. Par exemple, la CUQ profite du fait qu'elle est en même temps la capitale du Québec, ce qui implique la présence d'une fonction publique nombreuse et instruite. De plus, la fonction politique qu'exerce ce territoire lui permet de profiter d'une symbolique nationale de grande importance. Elle contribue largement à la définition de l'ensemble des représentations sociales, marquées fortement par la dimension internationale de la région. En effet, toute capitale entre

en compétition avec d'autres villes internationales et n'échappe pas aux courants qui structurent ces dernières. Parmi les facteurs les plus marquants, mentionnons la territorialisation poussée de l'économie et l'insertion de la dynamique urbaine dans les réseaux internationaux (Taylor et al. 1993: 97).

Par ailleurs, des facteurs géographiques, comme la situation par rapport aux grands centres métropolitains, la disponibilité ou l'absence d'une robuste infrastructure, la présence d'un bassin démographique important, jouent un rôle de premier plan dans l'évolution des 
dynamiques économiques du vaste territoire québécois. Si on peut généralement expliquer l'écart observé entre les quatre régions par des facteurs géographiques, historiques et politiques, il faut constater que le secteur privé intervient inégalement dans les territoires québécois, ce qui accentue l'écart existant. Les régions économiques périphériques du Québec ne semble pas attirer au même degré les investissements privés.

S'appuyant sur l'analyse de l'activité entrepreneuriale des territoires MRC, Proulx et Riverin classent les quatre territoires retenus pour analyse comme suit: Rivière-du-Loup est considérée comme un territoire à peu d'activité, marqué par un faible taux de création d'entreprises et un faible taux de fermeture d'entreprises. À des degrés différents, les trois autres territoires connaissent une activité plus intense, où on rapporte un taux élevé de création d'entreprises, mais aussi de fermetures d'entreprises (Proulx et Riverin : 1998). La CUQ et Rivière-du-Loup se démarquent par un dynamisme faible de l'entrepreneuriat; il est moyen au Saguenay et très élevé au Manicouagan (Proulx et Riverin : 1998). Lié à la nouvelle économie, le dynamisme entrepreunarial, comme l'avait remarqué Proulx depuis 1995, «se transforme en activités économiques, en emplois, en investissements, en valeur ajoutée, en impôts et en taxes» (Proulx 1995a : 17), bref, en prospérité.

Entre autres caractéristiques, la vitalité d'un espace se manifeste dans son degré d'industrialisation, dont l'importance relative est examinée ici dans chacun des territoires.

\subsubsection{L'activité économique}

Le niveau d'industrialisation d'un territoire est au cœur de ses dynamiques économiques, et il n'est pas sans intérêt de voir comment cette industrialisation se manifeste différemment dans les territoires concernés. Proulx (2002) a comparé les territoires MRC du Québec en fonction de leur niveau d'industrialisation. Sa réflexion est intéressante car elle aide à comprendre les dynamiques des territoires MRC en fonction de leur parcours sociohistorique, en fonction des actions publiques et des initiatives privées, et surtout de les situer les uns par rapport aux autres et par rapport au niveau national d'industrialisation. L'initiative a ceci d'original qu'elle explique le rôle du territoire et de "l'espace vécu" comme soutien de l'action, et qu'elle rend mieux compte ainsi du dynamisme des MRC du Québec. À cette fin, il a fondé son expérience sur l'analyse de certains indicateurs, notamment sur l'importance des activités 
manufacturières, (mesurée par le nombre d'entreprises manufacturières), sur les activités de recherche et développement, sur l'entrepreneuriat, sur l'animation et sur l'activité de divers réseaux. Ce sont là, selon l'auteur, les propriétés actives des territoires, qui donnent une idée raisonamblement précise du niveau d'industrialisation des territoires (Proulx 2002:257). Sur la base de ces indicateurs, l'auteur constate que le développement du territoire québécois connât trois phases principales, et il a calculé un indice qui permet de comparer entre eux les territoires MRC du Québec.

A la lumière des conclusions qui ressortent de cet exercice, les quatre territoires retenus dans le cadre de notre travail ont des niveaux différents d'industrialisation. L'auteur considère que la MRC Manicouagan se situe dans la phase 1 de l'industrialisation. Les territoires situés dans cette phase se distinguent par l'existence de «peu d'entreprises manufacturière (42 et moins). Il s'agit de territoires largement dominés évidemment par le secteur primaire [...] Ces territoires sont généralement bien pourvus en capacités innovatrices, en entrepreneuriat et en animation socioéconomique, mais faiblement dotés en réseautage» (Proulx 2002 : 258).

La MRC de Rivière-du-Loup se situe, selon les paramètres de Proulx en phase 2 de l'industrialisation. Les territoires classés en cette phase «accueillent entre 42 et 149 entreprises manufacturières. L'industrialisation de ces 45 territoires est bien en cours. [...] Ces territoires illustrent un indice moyen de potentiel de développement inférieur à la moyenne québécoise. Il apparaît que la formation professionnelle et l'entrepreneuriat font baisser leur indice global» (Proulx 2002: 261-262).

Par ailleurs, les deux autres territoires retenus dans le cadre de notre thèse, Fjore-du-Saguenay et CUQ, sont classés dans la phase 3 d'industrialisation. Les territoires classés dans cette catégorie «possèdent plus que 149 entreprises manufacturières. Ces 18 territoires sont en réalité en industrialisation avancée $[\ldots]$ Ces milieux sont mieux pourvus en R\&D, en formation et en entrepreneuriat) (Proulx 2002: 262). Comme le souligne l'auteur lui-même, le Fyore-du-Saguenay est le seul territoire périphérique du Québec classé dans la phase 3.

Bref, en présentant cette classification des territoires MRC du Québec en fonction d'indicateurs choisis avec soin, Proulx présente une vision originale de la capacité des territoires à soutenir le développement économique. De cette présentation, une affirmation 
sous-jacente se révelle: l'importance d'un espace vécu doit se mesurer par l'aptitude des acteurs à activer les particularités de leur territoire et on devrait appréhender cette activation en fonction de ses retombées positives sur l'économie régionale. C'est à la lumière de cette perception que l'auteur apporte sa propre réponse au questionnement que pose aux acteurs de développement la richesse plus au moins grande d'un territoire. Dès lors ce sont les acteurs qui sont responsables du devenir de leur territoire. Si les territoires réussissent, c'est parce que les acteurs ont su profiter de toutes les occasions qui leurs étaient offertes; dans le cas contraire il faudrait reconsidérer les stratégies collectives de développement. Proulx s'explique ainsi :

Cet exercice [...] permet d'effectuer une analyse territoriale du soutien au développement en nous appuyant sur des faits concrets. Si l'on considère l'évolution industrielle désirée dans la majorité des territoires, sauf dans les milieux touristiques, plusieurs territoires MRC apparaissent avoir les conditions actives aptes à les faire progresser d'une phase à l'autre de développement (Proulx 2002:262).

On ne peut guère espérer, toutefois, du développement sans investissements, ce qui n'a pas été inclus parmi les indicateurs considérés. L'auteur s'en explique ainsi : «le facteur du financement ne fut pas pris en compte puisque nous considérons que le capital est parfaitement mobile. Il pourra éventuellement être considéré comme une variable explicative» (Proulx 2002 : 262).

En somme, le classement des MRC, que Proulx a réalisé selon des critères objectifs, représente une contribution de grande utilité théorique ế scientifique. Il a souligné le potentiel de développement des territoires; il s'est employé autant à positionner les territoires MRC les uns par rapport aux autres et par rapport au niveau national, qu'à comprendre les facteurs sous-jacents qui leurs permettent de passer d'une phase d'industrialisation à une autre. Ce positionnement des $\mathrm{MRC}$ n'est évidemment pas définitif, puisque les situations évoluent, et qu'éventuellement une nouvelle étude deviendra nécessaire. Il serait souhaitable alors d'ajouter aux facteurs considérés un critère relatif à l'aptitude des acteurs à intégrer le nouvel ordre technologique. 
Après le rapide tour d'horizon qui précède, il y a lieu d'examiner plus en détail certaines données de nature à préciser davantage les caractéristiques de l'activité économique des territoires considérés.

\subsubsection{Caractéristiques particulières}

Nous avons vu, par le positionnement des territoires MRC du Québec, qu'ils se distinguent entre autres par leur niveau différent d'industrialisation. Si l'écart entre les territoires de notre étude peut s'expliquer par leurs niveaux d'industrialisation, d'autres caractéristiques, notamment géographiques, peuvent renforcer ou atténuer cet écart. Par exemple, Rivière-duLoup est un carrefour routier important. Cette situation permet aux composantes territoriales en place de profiter de certaines donnes exogènes, notamment l'ouverture sur d'autres territoires plus au moins actifs économiquement et socialement. D'un autre côté, le territoire de Manicouagan se situe au nord du Québec et souffre de l'absence d'infrastructures de transport adéquates qui pourraient le relier aux autres territoires du Québec. Comme avantposte de la conquête du territoire québécois, Manicouagan est l'un des territoires québécois les moins avantagés.

Bref, la section qui suit sert à nous rapprocher davantage des territoires retenus dans le cadre de cette thèse. Un bref retour à des données qualitatives et quantitatives supplémentaires semble nécessaire pour mieux comprendre comment les dynamiques économiques et sociales de développement des territoires MRC s'imbriquent dans leur cadre régional et national. II sera dès lors plus facile de comprendre le jeu des dynamiques de développement en place. Voici d'abord une vue d'ensemble de la situation de l'emploi et du marché du travail dans les quatre régions retenues, et ensuite dans les MRC qui nous concernent.

\section{Tableau 4: Variation du taux de l'emploi des 15-64 ans et variation du taux de chômage, les quatre réglons 1989-2001}

\begin{tabular}{|l|l|l|l|l|}
\hline & Taux d'emploi & Taux de chômage & \\
\hline & $1989-1994$ & $1994-2001$ & $1989-1994$ & $1994-2001$ \\
\hline Sagenay-Lac-S.-Jean & $-3,0$ & 8,8 & 2,9 & $-3,5$ \\
\hline Bas-Saint-Laurent & 2,2 & 0,0 & 1,9 & $-4,3$ \\
\hline Côte-Nord & $-2,9$ & 0,0 & 3,4 & $-1,4$ \\
\hline La Capitale & 0,3 & 3,1 & 3,5 & $-3,9$ \\
\hline Ensemble du Québec & $-2,7$ & 4,7 & 2,7 & $-3,6$ \\
\hline
\end{tabular}


Entre 1989 et 1994, seul le Bas-Saint-Laurent avait fait un gain appréciable d'emplois, Saguenay et Côte-Nord en perdant quelque $3 \%$, un peu plus que la moyenne québécoise. Entre 1994 et 2001 , la situation sinverse. On gagne partout des emplois, sauf au Bas-SaintLaurent qui demeure stationnaire. Le Saguenay fait un bond inattendu de 8,8\%. Entre 1989 et 1994 le chômage augmente dans les 4 régions, particulièrement à la Capitale et à la CôteNord, toutes deux dépassant la moyenne québécoise. Dans les années suivantes (1994 - 2001) le chômage diminue dans les 4 régions, se situant autour de la moyenne du Québec, excepté à la Côte-Nord, où la reprise s'avère plus lente. Le tableau suivant établit, pour 2001, une comparaison entre nos quatre régions, selon certains paramètres indicatifs de leur dynamisme économique.

Tableau 5: Population de 15 ans et plus selon la participation au marché du travaill, les quatre régions concernées, 2001

\begin{tabular}{|c|c|c|c|c|c|c|c|c|}
\hline \multirow[t]{2}{*}{ Régions } & \multirow[t]{2}{*}{ Total } & \multicolumn{3}{|c|}{ Population active } & \multirow{2}{*}{$\begin{array}{l}\text { Population } \\
\text { inactive }\end{array}$} & \multirow{2}{*}{$\begin{array}{l}\text { Taux } \\
\text { d'activité }\end{array}$} & \multirow{2}{*}{$\begin{array}{l}\text { Taux } \\
\text { d'emploi }\end{array}$} & \multirow{2}{*}{$\begin{array}{l}\text { Taux de } \\
\text { Chômage }\end{array}$} \\
\hline & & Total & Occupée & $\begin{array}{l}\text { En } \\
\text { chômage }\end{array}$ & & & & \\
\hline & & \multicolumn{3}{|l|}{$N$} & & \multicolumn{3}{|l|}{$\%$} \\
\hline Saguenay-Lac-S.J. & 225530 & 132795 & 114615 & 18185 & 92745 & 58,9 & 50,8 & 13,7 \\
\hline Bas-Saint-Laurent & 162655 & 95750 & 83090 & 12665 & 66890 & 58,9 & 51,1 & 13,2 \\
\hline Cote-Nord & 77915 & 48340 & 40725 & 7590 & 29570 & 62,0 & 52,3 & 15,7 \\
\hline Capitale-Nationale & 529130 & 337005 & 311320 & 25670 & 192120 & 63,7 & 58,8 & 7,6 \\
\hline Le Québec & 5832350 & 3742490 & 3434265 & 308220 & 2089865 & 64,2 & 58,9 & 8,2 \\
\hline
\end{tabular}

Source: Statistique Canada - Recensement du Canada, 2001

On remarque que la capitale nationale et Côte-Nord ont un taux d'activité proche de la moyenne québécoise (64\%), alors que Saguenay et Bas-Saint-Laurent ont un degré d'activité moins intense. Dans les trois régions périphériques, les taux d'emploi sont inférieurs d'environ 8 points à la moyenne québécoise (59\%); seule la Capitale réussit à égaler cette moyenne. Les taux de chômage dans les trois régions périphériques sont élevés, à plus d'une fois et demie la moyenne $(8 \%)$ du Québec. Par contre le taux de chômage est sous la moyenne dans la région de la capitale.

Le tableau suivant résume la situation de l'emploi dans les $4 \mathrm{MRC}$ de notre étude. On remarque que la CUQ fait mieux que la moyenne quant au taux d'emploi et au taux de 
chômage. Manicouagan et le Fjord conservent des taux de chômage d'environ $50 \%$ plus élevés que la moyenne québécoise.

Tableau 6: Population de 15 ans et plus selon la participation au marché du travail, les quatre MRC étudiées, 2001

\begin{tabular}{|c|c|c|c|c|c|c|c|c|}
\hline \multirow[t]{2}{*}{ MRC } & \multirow[t]{2}{*}{ Total } & \multicolumn{3}{|c|}{ Population active } & \multirow{2}{*}{$\begin{array}{l}\text { Population } \\
\text { inactive }\end{array}$} & \multirow{2}{*}{$\begin{array}{l}\text { Taux } \\
\text { d'activité }\end{array}$} & \multirow{2}{*}{ Taux } & \multirow{2}{*}{$\begin{array}{l}\text { Taux de } \\
\text { Chômage }\end{array}$} \\
\hline & & Total & Occupé & $\begin{array}{l}\text { En } \\
\text { chômage }\end{array}$ & & & & \\
\hline & & \multicolumn{3}{|c|}{$n$} & & \multicolumn{3}{|c|}{$\%$} \\
\hline Saguenay & 136260 & 80145 & 68775 & 10370 & 56120 & 58,8 & 51,2 & 12,9 \\
\hline Rivière-du-Loup & 25910 & 16190 & 14665 & 1525 & 9720 & 62,5 & 56,6 & 9,4 \\
\hline Manicouagan & 26975 & 16635 & 14605 & 2035 & 10340 & 61,7 & 54,1 & 12,2 \\
\hline CUQ & 424950 & 272080 & 252300 & 19775 & 152870 & 64,0 & 59,4 & 7,3 \\
\hline Le Québec & 5832350 & 3742490 & 3434265 & 308220 & 2089865 & 64,2 & 58,9 & 8,2 \\
\hline
\end{tabular}

Source: Statistique Canada - Recensement du Canada, 2001

Il n'est pas sans intérêt de jeter un coup d'œil sur les principaux secteurs d'activité qui occupent les populations de nos quatre territoires. C'est l'objet du tableau suivant.

Tableau 7: Population active de 15 ans et plus selon les industries les plus importantes, quatre régions concernées, 2001

\begin{tabular}{|c|c|c|c|c|c|c|c|c|c|c|c|}
\hline & & total & 11 & 21 & 23 & $31-33$ & $44-45$ & 61 & 62 & 72 & 91 \\
\hline \multirow[t]{2}{*}{ Saguenay } & $n$ & 132795 & - & - & 8125 & 21355 & 16580 & 9740 & 13425 & 8635 & - \\
\hline & $\%$ & 100,0 & - & - & 6,1 & 16,1 & 12,5 & 7,3 & 10,1 & 6,5 & - \\
\hline \multirow[t]{2}{*}{ Bas-S.L. } & $n$ & 95760 & 8795 & - & - & 12615 & 11330 & 6900 & 11265 & 6345 & - \\
\hline & $\%$ & 100,0 & 9,2 & & & 13,2 & 11,8 & 7,2 & 11,8 & 6,6 & \\
\hline \multirow[t]{2}{*}{ Côte-Nord } & $\bar{n}$ & 48320 & $=$ & 3320 & - & 7010 & 5395 & - & 5005 & 3575 & 3545 \\
\hline & $\%$ & 100,0 & - & 6,8 & - & 14,5 & 11,2 & - & 10,4 & 7,4 & 7,3 \\
\hline \multirow[t]{2}{*}{ Capitale } & $n$ & 336990 & - & - & - & 31155 & 37935 & 23215 & 38645 & 25 & 46085 \\
\hline & $\%$ & 100,0 & - & - & - & 9,2 & 11,3 & 6,9 & 11,5 & 7,6 & 13,7 \\
\hline \multirow[t]{2}{*}{ Le Québec } & $n$ & 3742490 & 101635 & 16890320 & 168355 & 640670 & 414905 & 240980 & 372090 & 223275 & 229410 \\
\hline & $\%$ & 100,0 & 2,7 & 0,4 & 4,5 & 17,1 & 11,1 & 6,4 & 9.9 & 6,0 & 6,1 \\
\hline
\end{tabular}

Source : Statistique Canada, Recensement du Canada, 2001

Les codes selon le Système de classification de l'industrie de l'Amérique du Nord (SCIAN): 11- Agriculture, foresterie, pêche et chasse; 21-Extraction minière et extraction de pétrole et de gaz; 23Construction;31-33 Fabrication ; 44-45Commerce de détail; 61-Services d'enseignement; 62- Soins de santé et assistance sociale; 72 -Hébergement et services de restauration; 91-Administrations publiques. 
On remarque d'abord que seuls le Bas-Saint-Laurent et la Cote-Nord ont un secteur primaire suffisamment important pour occuper une niche statistique, à $9 \%$ et $7 \%$ respectivement de la main-d'œuvre, la moyenne pour le Québec étant de 3,1\%.

Toutes les régions ont un secteur secondaire plus ou moins important. La Capitale a le pourcentage le plus faible: $9 \%$ de sa main-d'œuvre y est engagée. Viennent ensuite le BasSaint-Laurent: 13\%; la Côte-Nord: $14,5 \%$ et le taux le plus élevé: $22 \%$ au Saguenay. La moyenne est de $21,6 \%$ pour le Québec.

Dans les 4 régions, c'est le secteur des services qui occupe le plus de monde: $36 \%$ au Saguenay et à la Côte-Nord, 37\% au Bas-Saint-Laurent et $51 \%$ dans la Capitale. La moyenne pour le Québec est de 30,5\%. Le tableau suivant reprend les mêmes catégories, cette fois pour les MRC faisant l'objet de notre étude.

Tableau 8: Population active de 15 ans et plus selon les industries les plus importantes, les quatre MRC étudiées, 2001

\begin{tabular}{|c|c|c|c|c|c|c|c|c|c|c|c|c|}
\hline & & Total & 11 & 21 & 23 & $31-33$ & $44-45$ & 61 & 62 & 72 & 91 & Autres \\
\hline \multirow{2}{*}{ Saguenay } & $n$ & 80145 & - & - & 4955 & 12445 & 9795 & 6610 & 8020 & 5140 & - & 33185 \\
\hline & $\%$ & 100,0 & - & - & 6,2 & 15,5 & 12,2 & 8,2 & 10,0 & 6,4 & - & 41,4 \\
\hline \multirow{2}{*}{ Riv.-du-Lp } & $n$ & 16195 & 1025 & - & - & 2280 & 2135 & 1155 & 1815 & 1410 & - & 6380 \\
\hline & $\%$ & 100,0 & 6,3 & - & - & 14,1 & 13,2 & 7,1 & 11,2 & 8,7 & - & 39,4 \\
\hline \multirow{2}{*}{ Manicoua. } & $n$ & 16635 & - & 110 & - & 3780 & 1910 & - & 1955 & 955 & 985 & 6900 \\
\hline & $\%$ & 100,0 & - & 0,7 & - & 22,7 & 11,5 & - & 11,8 & 6,0 & 5,9 & 41,5 \\
\hline \multirow{2}{*}{ CUQ } & $n$ & 272075 & - & - & - & 22015 & 31395 & 19545 & 32105 & 20330 & 39660 & 107020 \\
\hline & $\%$ & 100,0 & - & - & - & 8,1 & 11,5 & 7,2 & 11,8 & 7,5 & 14,6 & 39,3 \\
\hline \multirow{2}{*}{ Québec } & $n$ & 3742490 & 101635 & 16320 & 168355 & 640670 & 414905 & 240980 & 372090 & 223275 & 229410 & 1621165 \\
\hline & $\%$ & 100,0 & 2,7 & 0,4 & 4,5 & 17,1 & 11,1 & 6,4 & 9,9 & 6,0 & 6,1 & 43,3 \\
\hline
\end{tabular}

Source : Statistique Canada, Recensement du Canada, 2001

Les codes selon le SCIAN: 11-Agriculture, foresterie, pêche et chasse; $21-$ Extraction minière et extraction de pétrole el de gaz ; 23-Construction; 31-33- Fabrication ; 44-45-Commerce de détail; 61-Services d'enseignement; 62-Soins de santé et assistance sociale; 72-Hébergement el services de restauration; 91-Administrațions publiques. 
Le secteur primaire est quasi inexistant dans ces MRC. Le peu de main-d'œuvre occupée dans ce secteur se trouve à Rivière-du-Loup: 1025 personnes, et 110 à Manicouagan. Au secteur secondaire, la CUQ occupe le plus faible pourcentage: $8 \%$ de sa main-d'œuvre, suivie de Rivière-du-Loup avec 14\%, puis du Fjord-du-Saguenay et de Manicouagan, avec environ $22 \%$ respectivement, ce qui correspond à peu près à la moyenne québécoise.

Comme on peut s'y attendre, le secteur des services occupe la plus grande place: environ $36 \%$ des travailleurs au Fjord-du-Saguenay et à Manicouagan, 40\% à Rivière-du-Loup; ces pourcentages équivalent presque à la moyenne québécoise. La CUQ se démarque nettement, avec plus de $52 \%$ de sa main-d'cuvre dans le secteur tertiaire.

Les tableaux qui précèdent sont éloquents et appellent peu de commentaires additionnels. À l'évidence, l'écart entre les régions se répercute assez clairement sur le dynamisme des MRC qui s'y trouvent. Il est remarquable toutefois, au moins pour les territoires qui nous occupent, qu'en général la MRC fait mieux que sa région. Ce qui n'empêche pas des disparités considérables entre ces MRC. Ces écarts se manifestent sur le plan institutionnel, le régionalisme fonctionnel, le régionalisme politique et l'exercice de la planification territoriale (Proulx 1995: 52-60).

Situées dans des zones géographiques aux caractéristiques dissemblables, nos quatre MRC disposent en conséquence de ressources et de conditions de vie différentes. Chaque territoire a sa propre histoire et a acquis des vocations qui lui sont propres, de même que des forces et des faiblesses particulières. Proulx remarque que les dynamismes de ces territoires évoluent différemment. Dans les années 1960, Québec et Chicoutimi étaient deux pôles primaires, alors que Rivière-du-Loup et Baie-Comeau-Haute-Rive étaient considérés comme des pôles secondaires. Toutefois grâce à la croissance de l'emploi dans la décennie 86-96, Québec devient un pôle à forte polarisation d'activités génératrices d'emplois; Rivière-du-Loup connaît une polarisation moyenne, tandis qu'à Chicoutimi et Baie-Comeau elle est faible ou nulle. (Proulx 2002: 247). 


\subsection{Synergies de développement}

Les perspectives économiques et sociales d'un territoire dépendent de plusieurs facteurs, notamment de la géographie, de l'histoire, de l'organisation interne, de la présence d'institutions fortes et variées. Il y a aussi des attitudes comme l'appartenance, la coopération, qui favorisent une gestion territoriale en fonction de l'intérêt commun.

Il est évident que la dynamique économique et socioculturelle de la CUQ est différente de celles qui ont cours dans les trois autres territoires. Le territoire de Saguenay se démarque toutefois par des caractéristiques propres et un sentiment d'appartenance fort, consolidé par son enchâssement dans le Royaume du Saguenay. Toutefois, l'histoire et la géographie y ont aussi induit un solide individualisme, qui ne favorise guère la coopération entre municipalités, institutions et organisations. En certains cas, le sentiment d'appartenance caractéristique du Saguenay a sans doute aidé le développement, mais souvent il a constitué plutôt un frein. Selon Proulx et Mévellic,

dans cette collectivité, la culture organisationnelle territoriale est tellement cristallisée autour d'une panoplie de positions identitaires, idéologiques, partisanes, centralisatrices, claniques et régionalistes ou autres que les dossiers territoriaux de développement économique n'arrivent que rarement à prendre assise solide (Proulx et Mévellic 2001 :357).

Depuis quelques décennies, les gouvernements accordent un intérêt particulier à l'échelon supralocal, que l'on utilise «plus systématiquement dans la gestion publique de biens et services collectifs à la population, aux travailleurs et aux entreprises» (Proulx 2003 : 87). Les quatre territoires MRC que nous avons choisis dans le cadre de cette analyse semblent jouer des rôles importants. Par leurs ressources variées et la place qu'elles occupent au sein de la nouvelle division spatiale des activités économiques, ces MRC participent différemment à la dynamique de leurs milieux respectifs. Cette participation montre comment chaque territoire MRC profite des forces spatio-économiques qui lui sont propres. Par exemple, Proulx constate que dans la nouvelle division spatiale des activités économiques, Rivière-du-Loup et Saguenay sont des centres de consommation qui profitent 
largement des flux économiques générés par la population (consommateurs) des centres d'extraction, des petits centres de services et d'autres lieux localisés sur le territoire [...]En réalité, les centres de consommation polarisent une bonne partie des achats de biens durables et semi-durables d'une population occupant un très vaste territoire, et les consommateurs qui résident dans ces centres dépensent très peu à l'extérieur. Cependant, les fux économiques (fuites) hors de ces centres de consommation et dirigés vers les centres de production sont énormes, car les marchés régionaux de consommation sont désormais largement occupés par des succursales de chaînes d'entreprises nationales ou internationales (Proulx 2002: 105).

Dans les régions, et peut être encore davantage dans les MRC qui «réussissent», on perçoit des synergies, nées plus au moins spontanément de besoins ressentis d'abord au palier local. Lorsque les circonstances s'y prêtent et portées par quelques personnes de vision, ces forces de collaboration s'étendent ế entraînent un maillage générateur de développement. Ce maillage résulte des interactions qui s'établissent entre les entreprises, les organismes d'appui au développement économique ou social, les institutions financières, etc.

Les organismes d'appui peuvent être de natures diverses, publique, privée ou mixte, tels que : les SADC, CLD, CLSC, SOLIDE, Chambre de commerce, etc. Les entreprises, les organismes, chacun participe selon sa vocation propre et sa capacité à l'avancement du désir collectif de progresser. Le fonctionnement et l'insertion des organismes publics ou semi publics dans le tissu socio-économique d'un territoire correspondent à des stratégies de développement qui varient selon les objectifs de la géo-économique dominante au Québec. Les politiques et stratégies de développement sont revues de temps à autre par les gouvernements successifs.

En pratique, le fonctionnement de ces organismes dépend de la vocation d'un territoire et de la place qu'il occupe aux échelons régional et supra-régional. Par exemple, on a dit de la MRC de Rivière-du-Loup qu'elle est le poumon économique du Bas-Saint-Laurent. On y compte en effet au-delà de 3000 entreprises agricoles, la plupart de petite taille toutefois, spécialisées dans la production laitière ( $65 \%$ des revenus agricoles), céréalière (15\%), bovine $(10 \%)$, ainsi que dans la culture de la pomme de terre. On y trouve aussi deux usines de pâtes et papiers, 6 scieries et 13 entreprises manufacturières. Il est évident que cette économie est d'une autre sorte que celle, par exemple, de la CUQ, qui domine dans le secteur tertiaire. 
C'est pourquoi, dans des territoires aussi peu semblables, les collaborations et la synergie qui s'y exercent sont nécessairement de nature différente.

La CUQ et le Fjord-du-Saguenay ont à leur avantage certains atouts : l'une le secteur des services, l'autre un secteur secondaire assez fort. Les deux autres territoires ont manifestement du rattrapage à faire. Beaucoup de jeunes les quittent, soit parce que l'emploi y est rare, soit que le cadre de vie ambiant ne les satisfait pas.

Faudrait-il pour autant classer Manicouagan et Rivière-du-Loup dans la catégorie des "régions perdantes" à perpétuité ? Il serait présomptueux de le faire. Il semble que la situation difficile des territoires périphériques du Québec peut s'expliquer par leur «passage direct à une phase de consommation de masse sans avoir vraiment traversé celle de la maturité économique basée sur la transformation industrielle des ressources et la diversification des activités économiques» (Côté et Proulx 2002 : 118). C'est pourquoi on parle de l'immaturité de ces territoires quand on pense à leur développement. Pourtant, il semble bien que la plupart des acteurs de ces territoires, attachés à leur terre, à leur lieu de souvenance, n'ont pas perdu courage et font encore confiance en l'avenir. Il serait peut-être plus juste de parler de "régions oubliées", que les pouvoirs publics semblent redécouvrir depuis quelque temps. En témoignent les récentes politiques régionales.

Quoi qu'il en soit, on comprend que les phases précédentes de l'évolution de nos sociétés imposaient la concentration, dans les grands centres urbains, des capitaux, de l'industrie et des services; que cette concentration s'est accompagnée non seulement du développement des métropoles, mais aussi de la marginalisation des territoires périphériques. Au Québec, cette situation s'est traduite par une immaturité de nos régions périphériques, occasionnée par «la distance des grands marchés, les coûts élevés du travail, la difficulté de retenir la maind'œuvre qualifiée, la fuite des profits et de l'épargne locale, le manque de savoir-faire» (Côté et Proulx $2002: 118$ ).

Ces dernières années, l'évolution des structures a toutefois modifié la situation. Les facteurs responsables de cette immaturité économique ne sont plus aussi contraignants qu'ils l'étaient auparavant. Porter a souligné que «la disponibilité de ressources telles que la main-d'œuvre et les matières premières est beaucoup moins importante pour la prospérité d'une nation que 
l'aptitude à les utiliser d'une façon productive, en mettant en cuvre un savoir-faire et une technologie pointus) (Leymarie et Tripier 1993:7). On est donc loin de l'époque où la compétitivité des territoires était liée à la disponibilité des ressources naturelles, de la maind'œuvre, etc. Les TIC poussent les acteurs implantés dans les territoires périphériques à développer un savoir-faire indéniable: «nous avons été très impressionnés par le niveau de savoir-faire avancé et de compétence technique de nos vis-à-vis, même dans les communautés les plus éloignées» (Polèse et Shearmur 2002: 60). Nous vivons dans un monde où la prospérité des territoires, comme celle des nations, dépend largement de leur capacité compétitive. Porter précise que «la compétitivité est maintenant basée sur la productivité, ou sur l'aptitude d'une nation à générer une production élevée et en constante augmentation à partir de chaque jour de travail et de chaque unité de capital investie» (Leymarie et Tripier 1993 : 7). La compétitivité repose désormais de plus en plus sur l'apport de la technologie de pointe et particulièrement sur l'usage des TIC. Mais cela dépend aussi largement des efforts qu'on y met.

Les éléments dégagés dans cette section présentent un portrait diversifié des quatre territoires étudiés. Profitant de ses avantages comme métropole et comme capitale, la CUQ se distingue par la domination du secteur tertiaire, un taux élevé de scolarité, d'emploi et un chômage relativement faible. Dans les autres territoires, le secteur primaire est très faible, le secteur secondaire dominant et les services moins diversifiés, ce qui rend leurs économies plus fragiles et passablement dépendantes des grandes villes.

Pour l'ensemble de ces territoires, l'état actuel du développement et les perspectives de progrès sont certes influencés par la géographie, la disponibilité des ressources naturelles, le parcours historique. On pourrait alors penser que le développement - ou son absence - répond à une sorte de logique immanente qui déterminerait sans retour le devenir d'un territoire. Peutêtre fondée dans le passé, une telle opinion n'est plus recevable. Chacun des territoires de cette étude se distingue par un ensemble de ressources, matérielles et humaines, que la vision et l'énergie des acteurs locaux, épaulés au besoin par les autorités politiques, devraient pouvoir développer à un niveau convenable.

Autrement dit, le différentiel de croissance entre territoires métropolitains et périphériques ne parait pas irrémédiable. Ce qui a changé, c'est une prise de conscience ces dernières années, 
par les autorités et une large partie de l'opinion publique, de la nécessité de ne pas laisser plus longtemops péricliter les régions périphériques au profit d'une urbanisation incontrôlée. C'est aussi l'ère informationnelle dans laquelle nous sommes entrés, grâce aux TIC, qui offre aux acteurs de ces territoires une quantité et une qualité d'information, en même temps qu'une facilité de communication auparavant difficilement accessibles, et qui peuvent donner au développement une impulsion nouvelle.

Désormais l'information - offre, demande, traitement, stockage - est également accessible partout et ne manque pas d'influencer les activités économiques et sociales. Certes, la seule information ne corrigera pas d'un coup le déséquilibre entre les territoires; elle contribuera cependant à réorienter les circuits d'influence en mettant les acteurs de tous les territoires sur un pied d'égalité. Voilà pourquoi il paraî opportun de consacrer un certain espace à une revue de l'information offerte à l'entrepreneur pour la conduite de ses affaires.

\section{3. À l'appui de l'entreprise, l'information disponible}

\subsubsection{L'information de sources privées}

Qu'elle émane de source privée ou publique, l'information influe sur la conduite de l'entreprise. Toutefois l'information issue de sources privées, parce que davantage ciblée, s'intègre plus facilement aux facteurs de production, au savoir-faire technologique, et facilite le développement des relations stratégiques de l'entreprise et donc des territoires.

Ratti montre l'utilité de cette information à partir de certains types de relations qui constituent l'espace de soutien de l'entreprise. Il constate que les sources d'information, avec bien d'autres facteurs, participent activement au développement des relations privilégiées de l'entreprise. Selon lui, ces sources d'information sont nécessaires pour l'établissement des

relations stratégiques de l'entreprise concernant ses partenaires, fournisseurs ou clients (échange privilégié d'informations, collaboration, partenariat, alliance, intégration partielle); et les relations stratégiques avec les acteurs de l'environnement territorial (institutions publiques, associations privées ou semipubliques) (Ratti 1992: 55). 
C'est pourquoi le dirigeant d'une entreprise, désirant raffiner ou renforcer sa capacité concurrentielle, a la plupart du temps d'abord recours à l'information de source privée, qui lui paraît plus immédiatement pertinente à ses projets.

Par leur diversité et leur proximité, les sources d'information privées jouent un rôle important dans l'élaboration des stratégies d'affaires et des activités dans un territoire, et c'est surtout à partir de sources privées que se concrétise l'innovation dans une entreprise. Plus que les sources publiques, les sources privées sont nécessaires à qui veut prévoir les contraintes, les obstacles, de même que les perspectives de développement. C'est en observant les initiatives menées dans le secteur privé que l'on comprend le mieux le cheminement d'une entreprise à la recherche d'une plus grande efficacité. Porter constate que

les opérations conduites par le secteur privé avec la participation de l'administration ont plus de chances de réussite que celles contrôlées par les pouvoirs publics. Les entreprises sont généralement mieux placées que l'État pour dire à quels obstacles et contraintes elles se heurtent. De plus, une action dirigée par le secteur privé aura un contenu politique moindre et bénéficiera de la capacité de réalisation souvent supérieure du secteur privé (Porter $1999: 274$ ).

S'il est vrai qu'une utilisation optimale des ressources est généralement mieux réussie dans le secteur privé, on conçoit que plus l'information offerte est pertinente à l'activité concernée, plus elle a d'importance pour l'entreprise. Mais la circulation de l'information dans les milieux d'affaires répond à une dynamique complexe qui découle

des conditions qui facilitent le flux d'informations ou qui déverrouillent l'information, rendant la coordination plus aisée en instaurant la confiance et en atténuant les divergences d'opinion entre firmes (liées horizontalement ou verticalement) quant à leurs intérêts économiques. En voici quelques exemples : facilitation du flux d'information (relations personnelles tenant à la camaraderie d'école ou de service militaire; lien au sein de la communauté scientifique ou au sein d'associations professionnelles; liens communautaires tenant à la proximité géographique; syndicats interprofessionnels regroupant des grappes ; croyances en des valeurs telles que la continuité ou les relations à long terme (Porter 1993 : 168).

Les mécanismes par lesquels l'offre privée d'information agit sur le développement des affaires sont nombreux et fluctuants. Ils reposent d'abord sur les besoins exprimés d'un 
collectif impliqué dans un processus de prise de décision. Comme l'entreprise cherche naturellement les stratégies les plus aptes à la faire profiter des occasions qui se présentent, l'expression des besoins mène à des interrogations concernant l'information disponible. Souvent, la tâche lui est facilitée par le contexte entourant les éléments concernés dans la prise de décision, tels que

la proximité, les liens technologiques ou client/foumisseur et l'existence de relations personnelles et sociales répétitives. (Toutes ces conditions facilitent la circulation des informations délicates). Les informations sur les besoins actuels des clients sont l'un des grands avantages des grappes à cet égard. Elles comprennent souvent des acheteurs de haut niveau, et d'autres participants font circuler les informations dont ils disposent sur les besoins des clients (Porter 1999:226).

Dans la pratique, une entreprise adopte des stratégies différentes pour s'adapter aux limitations imposées par les circonstances. Ainsi, lorsqu'il s'agit d'affronter les contraintes d'un marché de plus en plus concurrentiel, il importe qu'elle puisse s'appuyer sur de solides éléments porteurs de ses propres modes d'action, de réaction et d'interaction. C'est alors que l'information prend tout son sens et son utilité, celle de source privée particulièrement parce que plus immédiatement pertinente, celle aussi circulant entre pairs, par exemple dans les réseaux d'entreprises. Il est manifestement impossible de répertorier toutes les sources d'information privées. Les principales comprennent la presse grand-public et spécialisée, les médias électroniques (radio, télévision, Internet), les expositions, les missions économiques, etc.

Les journaux locaux et régionaux constituent une source importante d'information. Ils apportent une information adaptée au contexte local et régional qu'ils connaissent bien. Leur forme, le ton qu'ils utilisent et, évidemment, leur contenu donnent une bonne indication des dynamiques économiques, sociales et culturelles en place. Ils véhiculent une information qui touche presque tous les domaines car ils sont un espace que les acteurs économiques et sociaux utilisent pour informer, influencer et offrir biens et services.

Les stations de radio et de télévision donnent une autre image de l'offre privée d'information. Alors que la plupart des stations de radio sont étroitement liées aux conjonctures locales, car utilisées pour véhiculer une information qui touche tout le monde, pour annoncer 
l'organisation de divers évènements et activités, les stations de télévision prennent la plupart $\mathrm{du}$ temps une dimension régionale, voire plus large, et desservent une clientèle régionale. Certes, les stations de radio et de télévision apportent des réponses à des questions que soulève la vie quotidienne des principaux acteurs et des simples citoyens. Ces stations participent largement à faire circuler l'information et à stimuler les dynamiques économiques et socioculturelles.

Les expositions à caractère scientifique sont des occasions qui facilitent la vulgarisation des connaissances et du savoir scientifique. Les acteurs peuvent s'y mettre au courant des inventions et des découvertes les plus récentes et se familiariser avec les nouvelles technologies et techniques utilisées dans leur secteur d'activité. Pratiquement tout dirigeant d'entreprise, quel que soit son domaine d'activité, peut adapter à ses besoins particuliers les connaissances et le savoir offerts lors de ces expositions.

En plus de ces expositions à caractère scientifique, on constate la tenue de plusieurs foires commerciales, qui sont d'un intérêt certain pour les entreprises et le développement économique. Notamment elles permettent aux entreprises de contacter leurs fournisseurs et de voir où sont rendus leurs concurrents.

\subsubsection{L'information de sources publiques}

On pense spontanément au gouvernement et à ses ministères lorsqu'il est question de sources publiques d'information. D'autres organismes sont aussi fournisseurs occasionnels d'information, notamment le secteur parapublic. Toutefois l'État est de loin le plus abondant fournisseur. C'est pourquoi, à toutes fins utiles, l'information émanant de ces organismes est ici assimilée à une source étatique.

$\mathrm{Si}$, pour l'entreprise, l'utilité de l'information d'origine privée est indéniable, l'accélération du temps a donné un relief fortement accru au rôle de l'État dans la diffusion de l'information. C'est d'abord que les pouvoirs publics ont continuellement des renseignements de toutes sortes à communiquer aux acteurs de développement. C'est aussi que, poussés par la conjoncture, les États ont vu l'utilité d'entrer dans le jeu économique en posant des balises visibles. 
Le rôle des pouvoirs publics est généralement admis, mais ses manifestations ne s'expriment pas toujours de la même façon. Chaque État cherche à intervenir d'une manière ou d'une autre, soit pour inciter les acteurs à profiter d'une conjoncture favorable, soit pour les aider à atténuer les conséquences adverses d'un ralentissement. L'action étatique subit cependant des pression de toute sorte, souvent en sens opposé: des syndicats, des patrons, des groupes de pression et autres organismes; mais les influences les plus décisives viennent principalement des forces économiques et sociales, qui parviennent difficilement à s'adapter aux vagues du changement. Les secousses de l'économie de l'information illustrent bien ce déplacement des rapports de forces.

Conscients de limportance grandissante des avancées scientifiques et technologiques sur les dynamiques sociétales, des économistes font ressortir l'importance de l'information et son rôle essentiel pour la vitalité de l'espace public. Cet espace «est un fort lieu de matérialisation, on pourrait dire de 'communauté en débat") (Sicard et Besnier 1998 : 152). Il est le théâtre de profonds changements culturels: d'une époque relativement statique, on file vers une période aux contours encore flous, aux traits mouvants et composites.

Au Québec, la pensée sociale-démocrate a inspiré, depuis la Révolution tranquille, l'action des gouvernements successifs et amené l'État, avec l'accord général de la population, à intervenir dans la plupart des domaines. Il en est résulté ce qu'on a appelé le 'modèle québécois' et l'information émanant des pouvoirs publics a eu naturellement tendance à s'aligner sur les principes qui le caractérisent. Ce modèle se trouve aujourd'hui à la croisée des chemins, entre l'attachement à un passé sécurisant et un avenir incertain.

L'éventail de l'offre publique d'information est large et couvre la plupart des aspects de la vie civile: réglementaire, économique, sanitaire, culturel, etc. À noter qu'il s'agit ici de l'offre d'information, à distinguer de la demande, lesquelles ne coïncident pas toujours exactement. Par ailleurs, loin de minimiser l'importance de la documentation produite par les ministères à vocation sociale: santé, éducation, culture, etc., le cadre de cette recherche imposait de s'en tenir à des documents touchant l'économie.

L'offre d'information de nature économique concerne dans une forte proportion l'information stratégique, c'est-à-dire une information liée exclusivement à son utilité. L'utilité d'une 
information peut toutefois être occultée par le fait de technologies en perfectionnement constant résultant d'avancées scientifiques, et aussi par la facilité que permet la technologie de créer des formes variées de sociabilité et de socialité.

On en arrive ainsi à l'aphorisme, à première vue paradoxal, selon lequel «every business is an information business" (Evans et Wurster $2000: 21$ ), qui présume l'existence d'une logique originale attribuant nécessairement à une information une utilité particulière. De fait, les rapports à linformation deviennent plus apparents si on les situe entre la satisfaction des besoins de connaissance et l'élargissement du monde informationnel:

un terme qui renvoie à une forme particulière diorganisation sociale, dans laquelle la création, le traitement et la transmission de linformation deviennent les sources premières de la productivité et du pouvoir, en raison des nouvelles conditions technologiques apparaissant dans cette période historique-ci (Castells $1998: 42$ ).

Les documents d'information produits par l'État québécois et d'autres organismes publics relèvent principalement de la dernière catégorie, i.e. des applications de type 'information connaissance', 'services' et 'information - événements'. Ces publications sont recensées plus loin. En fait le gouvernement québécois s'efforce le plus souvent d'aller bien au-delà de la simple information. L'un des points forts du 'modèle' est cette collaboration étroite qui existe généralement entre le gouvernement et un grand nombre d'organismes, qui cherchent ensemble à structurer, de manière originale, l'économie québécoise.

Cette collaboration amène ces organismes à mettre en question les vues gouvernementales et incite le gouvernement à modifier, le cas échéant, ses orientations ou à en adopter de nouvelles. Répondant à des critiques selon lesquelles l'action de l'État québécois exige une trop nombreuse bureaucratie, Julien indique que le marché, laissé à lui-même, génère lui aussi de nombreux bureaucrates dans les grandes entreprises. Comme le marché, «le gouvernement fait de bons coups et des erreurs, qu'il faut compenser (...) si possible en recourant à différentes formes de concertation entre représentants des secteurs privé et public, 'auxquels se joindront les syndicats (...) comme essaie de le faire de façon originale le modèle québécois» (Julien 2001). 
De temps à autre l'un ou l'autre ministère publie, pour information ou consultation publique, un document qui, après discussion avec les organismes professionnels et autres intéressés, peut devenir une politique, un programme ou un plan d'action. Dans le domaine qui touche directement le sujet de cette recherche, et en réponse à la conjoncture, le gouvernement créait en 1998 le ministère de la Recherche, de la Science et de la Technologie (MRST), dont le titulaire devenait membre d'office du comité ministériel des Priorités. On procédait en même temps à la création du comité ministériel de la Recherche, de la Science et de la Technologie. Il s'agissait d'un pas important, comme en témoigne la mission du nouveau ministère:

promouvoir la recherche, la science, la technologie et linnovation en favorisant la synergie des différents acteurs intervenant dans ces domaines, par l'établissement de mécanismes facilitant leur concertation et lintégration de leurs actions (...). Cette mission comporte l'élaboration et la mise en œuvre d'une politique de la recherche, de la science, de la technologie et de linnovation, en collaboration avec, notamment, les partenaires des milieux universitaire, collégial, industriel et gouvernemental (http://www.mrst.gouv.gc.ca/ fr/struct.htm)

Une collaboration suivie entre le MRST et les autres instances gouvernementales allait également de soi, surtout avec le ministère de l'Industrie et du Commerce, dont la mission est de «contribuer au développement économique du Québec, en intensifiant la compétitivité des entreprises et le développement des marchés, aux fins d'appuyer la création d'emplois» (http://www.mic.gouv.qc.ca/ministere/mission.html\#mission). Le MRST s'est avéré un acteur important dans le champ de la recherche - développement par les synergies qu'il a suscitées et l'information qu'il a rendue disponible. En 2001 le ministre présentait une politique sur la R-D :Savoir changer le monde, qui fut bien accueillie. Par la suite, le ministère recevait un budget de 370 millions de dollars pour la mise en cuvre de cette politique. Lors de la récente réorganisation de l'appareil gouvernemental, le MRST est devenu le ministère du Développement économique et régional, mais l'essentiel de sa mission a été conservé.

Les ministères, les organismes d'État, les institutions parapubliques, sont reliés par voie électronique et ont des sites sur la Toile, pour rendre l'information facilement accessible non seulement sur papier mais par tous les supports existants. 11 en résulte des avantages pour tous les intéressés : «des économies (salaires, déplacements, etc.); des rêductions de délais pour satisfaire aux exigences gouvernementales; une plus grande facilité d'accès aux services gouvernementaux (dans le temps et dans l'espace); la personnalisation des échanges; 
l'amélioration du suivi des dossiers; la qualité et la constance de l'information reçue)(Gosselin et Simon $2000: 11$ ).

Cette revue des éléments constituants de l'offre d'information en montre la grande diversité et éclaire sur leur finalité. Elle suggère de plus quelques pistes de réflexion. Les conjonctures associées à la mondialisation ont des effets différenciés, positifs ou négatifs, dans les territoires concernés et la qualité et la disponibilité de l'information peuvent faire la différence. Or, il n'est pas évident que, sans une autorité coordonnatrice, l'acteur de développement ou même la collectivité soient en mesure de construire des systèmes informationnels répondant adéquatement au changement continu, voire à le précéder. C'est pourquoi le gouvernement du Québec et les institutions qu'il soutient ont pris l'initiative et produit une offre d'information de nature à garder les entreprises québécoises en bonne place dans la société dite "en réseaux". On a ensuite voulu pousser un cran de plus. Dans un souci d'efficacité, les ministères et organismes du gouvernement sont tenus, depuis le premier avril 2001, de publier leurs propres déclarations de service aux citoyens. La loi sur l'administration publique oblige dorénavant toutes les organisations de la fonction publique à publier un document dans lequel elles décrivent leur mission, les services offerts à la population, et en fin d'année une reddition de comptes quant à l'atteinte de leurs objectifs.

\section{Conclusion}

Partant des territoires qui nous préoccupent, nous avons montré qu'ils profitent, à des degrés différents, d'une dynamique d'industrialisation dictée largement par les ressources dont dispose chaque territoire, mais aussi par l'habileté des acteurs individuels et collectifs à stimuler les dynamiques du développement. Les caractéristiques générales des territoires étudiés montrent qu'ils sont souvent prisonniers d'un parcours sociohistorique qui continue d'influencer les attitudes et les comportements des acteurs. À cause d'un niveau inégal d'industrialisation et de certaines caractéristiques d'ordre géographique et social̆, ces territoires n'offrent pas le même potentiel aux acteurs en place.

L'offre et la demande d'information indiquent que les territoires du Québec profitent désormais d'une source supplémentaire de richesse. Cette ressource, l'information, accrô̂t la capacité des acteurs à activer les propriétés favorables du territoire qu'ils partagent. 
L'information est considérée comme une source de richesse car elle offre les conditions nécessaires à l'activation de ces propriétés. Si les acteurs sont bien guidés, savent mener judicieusement leurs affaires, acquièrent la formation qui peut leur manquer et se tiennent au courant des données scientifiques qui les concenrnent, alors se trouve renforcée leur aptitude à profiter des caractéristiques de leur territoire et à en stimuler le développement. La consultation et la collaboration avec les diverses entités scientifiques et autres sources d'information consolident cette aptitude.

Bref, s'il importe que l'offre d'information soit de nature telle qu'elle contribue efficacement à l'insertion de la société dans la nouvelle économie, il est tout aussi important que les acteurs sachent en tirer profit. Car comme jamais auparavant, la conjoncture exigera d'eux compétence et vigilance. Le succès d'un territoire et de sa population dans la société en réseaux dépend de plusieurs facteurs, dont une information à jour et commodément disponible, mais surtout de la capacité des acteurs de développement de se l'approprier et de l'utiliser efficacement. 


\section{CHAPITRE 6}

\section{PROBLEMATIQUE ET ORIENTATION DE LA RECHERCHE}

Les acteurs de développement entretiennent avec leur environnement socio-économique et technologique des relations complexes. Une abondante littérature scientifique montre l'importance des phénomènes associés au changement technologique, de même que les effets des technologies nouvelles sur le fonctionnement des relations sociales, en particulier. De plus, les dynamiques économiques et sociales relatives à l'instauration de divers systèmes d'information sont essentielles pour l'innovation dans les activités économiques. Ainsi on souligne l'importance des mécanismes touchant la recherche, la transmission, la sélection, la transcription, la transformation et le contrôle de l'information, ainsi que les effets de l'information sur les dynamiques économiques et sociales de développement de milieux divers.

Par ailleurs, les relations des entreprises avec les autres institutions de leurs milieux incitent à accorder un intérêt particulier aux relations avec les concurrents, les clients, les fournisseurs, les consultants, les instituts privés et publics de recherche, les universités. Ces relations se fondent principalement sur la nature et le fonctionnement des différents canaux de collecte de l'information. De ce point de vue, il convient de souligner l'importance de plusieurs types de relations : formelles et informelles avec les clients, avec les fournisseurs; les relations avec les 
agences publiques locales et d'autres organisations: journaux, foires, banques de données, conférences, associations professionnelles; et les relations professionnelles.

Enrichi des propos développés dans les chapitres précédents, nous avons voulu rendre compte des dynamiques économiques de développement dans les quatre territoires de cette étude. 11 a donc paru à propos de concentrer cet examen sur les points suivants : les besoins informationnels qui peuvent se manifester sous forme d'une recherche de soutien externe, permettant de combler des lacunes internes ou de répondre à des besoins particuliers; les systèmes internes de communication, de classement et de traitement de l'information; les activités extérieures aux milieux de travail; les moyens d'interaction choisis par les dirigeants d'entreprises; les différents usages de l'Internet et du Web.

\subsection{Les variables}

Nous avons retenu un nombre important de variables, réparties en deux grands groupes. Le premier groupe permet de saisir les besoins, la demande et la circulation de l'information. $Y$ sont réunies les variables relatives aux sources d'information et aux moyens d'interaction, les types d'information et les activités extérieures, les systèmes de communication et les systèmes de classement de l'information. Le deuxième groupe concerne l'innovation technologique. Y sont rattachées les variables concernant le soutien extérieur que recherchent les entreprises, le branchement à l'Internet, l'usage du Web, le commerce électronique et l'appropriation du matériel informatique et de divers logiciels.

Tout en présentant l'ensemble de ces variables dans les paragraphes qui suivent, nous tenterons d'en montrer la pertinence par rapport à la question à l'étude. De même, nous présenterons la codification adoptée pour la manipulation des données au moyen du logiciel SPSS.

\subsubsection{Les besoins, la demande et la circulation de l'information}

Les entreprises recourent à diverses sources pour se procurer l'information nécessaire à la gestion de leurs opérations. Cette information peut provenir des fournisseurs de l'entreprise, de ses clients, des centres de recherche, des universités. Une information importante peut 
provenir des instances gouvernementales, (provincial ou fédéral). Elle provient aussi des foires et des expositions et des sociétés-conseils. Les entrepreneurs peuvent aussi se renseigner en visitant des entreprises canadiennes ou des entreprises à l'étranger. Les sociétés de développement, de même que les chambres de commerce, sont aussi des sources d'information et il en va de même pour les revues et journaux spécialisés, ainsi que pour les médias régionaux. De plus, les entrepreneurs peuvent toujours mettre à profit leurs contacts personnels pour s'informer.

\subsubsection{Les sources d'information}

Le questionnaire comportait une question principale qui a permis de saisir les sources d'information privilégiées par les dirigeants d'entreprises. C'est dans un contexte largement dominé par l'information et son économie que les dirigeants d'entreprises cherchent à se procurer l'information stratégique. Il leur faut impérativement veiller à disposer de l'information pertinente, sans laquelle leurs affaires deviennent risquées. En général nous avons identifié les sources les plus utilisées par ces dirigeants, afin de voir jusqu'à quel point ils les utilisent. Des indices de 1 à 4 ont été attribués à chaque groupe de réponses. Ainsi, l'indice 1 : très souvent; 2 : souvent; 3 : peu souvent; $4:$ jamais. Les variables mesurées sont présentées ci-dessous, avec le code qui leur a été attribué :

$\begin{array}{ll}\text { sfourn }= & \text { Les fournisseurs des entreprises; } \\ \text { sclien }= & \text { Les clients de l'entreprise; } \\ \text { scentr }= & \text { Les centres de recherche; } \\ \text { sniver }= & \text { Les universités; } \\ \text { sgoufe }= & \text { Le gouvernement fédéral; } \\ \text { sgoupr }= & \text { Le gouvernement provincial; } \\ \text { sfoire }= & \text { Les foires et expositions; } \\ \text { sdcusp }= & \text { Les sociétés-conseils ; } \\ \text { svican }= & \text { Visites d'entreprises canadiennes; } \\ \text { sviset }= & \text { Visites d'entreprises à l'étranger; } \\ \text { sdcusp }= & \text { Documentation spécialisée; } \\ \text { ssocon }= & \text { Visite de sociétés-conseils; } \\ \text { ssodev }= & \text { Sociétés de développement } \\ \text { srevjs }= & \text { Revues et journaux spécialisés; } \\ \text { srevjr }= & \text { Revues et journaux régionaux; } \\ \text { sconpe }= & \text { Contact personnel; } \\ \text { scham }= & \text { Chambre de commerce. }\end{array}$




\subsubsection{Les moyens d'interaction}

L'examen des moyens d'interaction semble important pour comprendre comment les dirigeants d"entreprises les utilisent dans leurs contacts quotidiens. Dans ce cas, le choix a été fixé sur des moyens d'interaction plutôt traditionnels, comme les activités de face-à-face, la place publique. De même, comment ces dirigeants utilisent des moyens d'interaction relativement modernes, comme le téléphone et la télécopie. Enfin, nous avons voulu saisir comment ils utilisent le courriel. Dans tous ces cas, nous avons cherché à mesurer l'utilisation de ces moyens tant pour émettre l'information que pour la recevoir. Les différents moyens d'interaction ont été classés selon des indices variant de 1 à $5: 1:$ moins de 5 fois par jour; 2 : entre 5 et 10 fois par jour; 3 : entre 10 et 15 fois par jour; $4:$ entre 15 et 20 fois par jour et 5 : 20 fois et plus par jour. Les moyens d'interaction et leur codification sont présentés cidessous:

TEREC = Téléphone comme moyen de réception d'information;

TEEMT = Téléphone comme moyen d'émission d'information;

TECREC = Télécopie comme moyen de réception d'information;

TELCEMT = Télécopie comme moyen d'émission d'information ;

FACEREC = Les activités de face-à-face comme moyen de réception d'information;

FACEEMT = Les activités de face-à-face comme moyen d'émission d'information;

PUBLREC = La place publique comme moyen de réception d'information ;

PUBLEMT = La place publique comme moyen d'émission d'information;

COURREC $=$ Le courriel comme moyen de téception d'information ;

COUREMT $=$ Le courriel comme moyen d'émission d'information.

\subsubsection{Les types d'information}

Si les entreprises utilisent très largement l'information stratégique, alors il faut savoir quel type d'information est la plus recherchée. Les besoins en information varient d'un territoire à l'autre. Une information, qui peut être stratégique pour les entreprises des territoires périphériques, peut n'avoir aucune utilité dans une grande métropole. L'aspect stratégique de l'information relève des exigences locales et régionales. C'est pourquoi nous avons cherché à saisir le type d'information que les dirigeants d'entreprises recherchent dans les quatre territoires retenus pour cette étude. Les réponses à la question portant sur le type d'information ont été classées selon des indices de 1 à 4 , qui ont été attribués à chaque groupe de rêponses. Ainsi, l'indice 1 : très souvent; 2 : souvent; $3:$ peu souvent; 4 : jamais. Voici les variables retenues dans ce cas: 
INFOCONJ = La conjoncture ;

INFCONC = La concurrence;

INFNVPRO $=$ Les nouveaux produits ;

INFEXPOR = Les exportations ;

INFSCIEN = L'information scientifique ;

INFTECHO = L'information technologique ;

INFTECHI = L'information technique;

INFNOJU = L'information d'ordre normatif, juridique ;

INESECUR= La sécurité;

INFRESPU = Les ressources publiques;

INFOMEDGE = Les modalités de gestion .

\subsubsection{Les activités extérieures}

La participation des dirigeants d'une entreprise à des activités extérieures est importante pour la réussite des stratégies de la firme. Ces activités favorisent en effet des échanges précieux entre ces dirigeants et leurs pairs, et avec les autres acteurs du même territoire. La participation à ces activités renforce l'enracinement de l'entreprise dans son milieu et lui permet de profiter d'un cadre social approprié. C'est pour cette raison que nous avons cherché à connaître les types d'activités qui intéressent les dirigeants d'entreprises. Les réponses aux questions portant sur les activités organisées à l'extérieur des entreprises sont classées selon des indices de 1 à 4 . Ainsi, l'indice 1 : très souvent ; 2 : souvent; 3 : peu souvent; 4 : jamais. Les activités extérieures sont notées comme suit :

\begin{tabular}{|c|c|c|}
\hline ACTCARI & $=$ & Causes caritatives ; \\
\hline ACTEVNMM & $=$ & Évènements mondains; \\
\hline ACTEVNMS & $=$ & Les évènements spéciaux; \\
\hline ACTEXPO & $=$ & Les expositions ; \\
\hline ACTERECO & $=$ & Les activités de reconnaissance ; \\
\hline ACTENVIR & $=$ & Les activités liées à l'environnement ; \\
\hline ACTESPOR & $=$ & Les activités sportives; \\
\hline ACTECLUS & $=$ & Les activités organisées dans clubs sociaux et \\
\hline ACTEREPA & $=$ & Les repas. \\
\hline
\end{tabular}

\subsubsection{Les systèmes de communication}

Les entreprises utilisent plusieurs systèmes de communication. Parmi les systèmes traditionnels, on trouve le tableau de nouvelles, le bulletin interne, la réunion du personnel. Mais des entreprises recourent davantage à des systèmes nouveaux, comme l'Intranet. La 
réponse à la question concernant ces systèmes est dichotomique : les dirigeants indiquent $(0)$ lorsqu'ils ne recourent pas à tel système et (1) lorsqu'ils l'utilisent. Les variables qui réfèrent aux systèmes internes de communication sont notées de la façon suivante :

$\begin{array}{lll} & \\ \text { SYSCOMBUL } & = & \text { Bulletin interne } \\ \text { SYSCOMTN } & =\text { Tableau de nouvelles } \\ \text { SYSCOMIN } & = & \text { Intranet; } \\ \text { SYSCOMRP } & =\text { Réunion du personnel } \\ \text { SYSCOMHC } & =\text { Horaire de mise en commun; } \\ \text { SYSCOMAU } & =\text { Autres. }\end{array}$

\subsubsection{Le classement de l'information}

Les entreprises utilisent plusieurs systèmes de classement de l'information. Comme pour les systèmes de communication, elles utilisent des systèmes traditionnels, comme le classement par sujet, par date, par projet. D'autres sont à base d'une technologie de pointe, comme le classement informatique, les banques de données, etc. La réponse à la question concernant ces systèmes est dichotomique : les dirigeants indiquent (0) lorsqu'ils ne recourent pas à tel système et (1) lorsqu'ils l'utilisent.

\begin{tabular}{|c|c|c|}
\hline CLASUJET & $=$ & Classement par sujet; \\
\hline CLASPROJ & $=$ & Classement par projet; \\
\hline CLASDATE & $=$ & Classement par date ; \\
\hline CLASIFOR & $=$ & Classement informatique ; \\
\hline CLASBDON & $=$ & Classement banque de données ; \\
\hline CLASBIBL & $=$ & Classement dans les bibliothèques de l'organisation ; \\
\hline ClasaUCU & $=$ & Aucun classement; \\
\hline CLASAUTR & $=$ & Aucune structure de classement. \\
\hline
\end{tabular}

\subsubsection{L'innovation technologique}

Il y a plusieurs façons de saisir linnovation technologique. Dans le cadre de cette recherche, nous avons abordé cette question à partir du soutien extérieur que les dirigeants d'entreprises vont chercher à l'extérieur de leur organisation : le branchement à l'Internet, l'usage qu'ils font 
du Web et le commerce électronique. Enfin, nous nous sommes intéressé aux composantes du matériel informatique et aux logiciels.

\subsubsection{Le soutien extérieur}

Les transformations profondes qui touchent nos sociétés et économies s'accompagnent de la généralisation des composantes technologiques de pointe. Les entrepreneurs doivent souvent recourir à un soutien extérieur pour résoudre les problèmes qui surviennent occasionnellement. Le recours à ce soutien extérieur est donc un indice qui montre le genre de problème qu'impose l'appropriation d'une technologie de pointe. La réponse à la question concernant ces soutiens est dichotomique : les dirigeants indiquent (0) lorsqu'ils ne recourent pas à tel soutien, (1) lorsqu'ils l'utilisent.

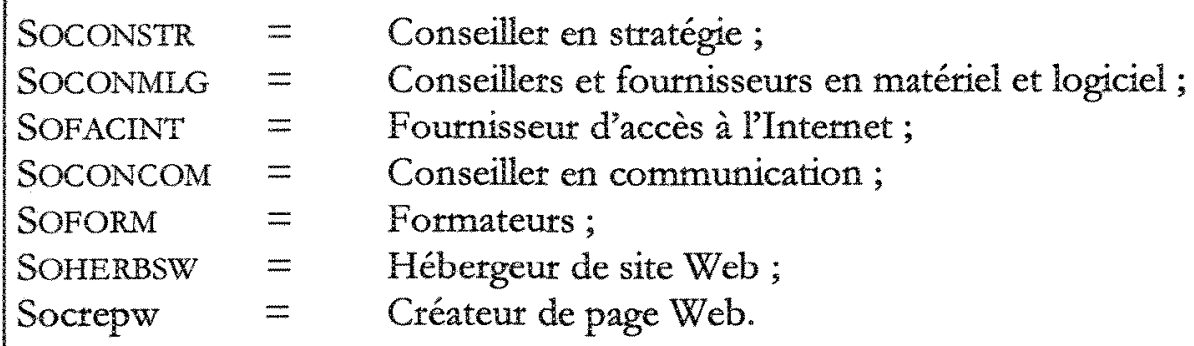

\subsubsection{Le branchement à l'Internet}

Le branchement à l'Internet est un indicateur fort intéressant, qui témoigne de l'aptitude des acteurs à s'insérer dans la société en réseau. Nous avons posé la question pour savoir si et depuis quand l'entreprise est branchée à l'Internet. Les réponses à cette question sont classées selon des indices de 1 à 5 . Ainsi : $1=$ branché depuis moins de 6 mois ; $2=$ Entre 6 et 12 mois; $3=$ Plus d'un an ; 4= Plus de deux ans; $5=$ Pas branché.

\subsubsection{L'usage du Web}

Nous avons voulu cerner ce que les dirigeants d'entreprises font du Web. Cet usage est un indicateur de la capacité des acteurs à intégrer le nouvel ordre technologique. 11 montre aussi leur capacité à élargir leur savoir, améliorer leur savoir-faire et à innover. La réponse à la 
question concernant ces systèmes est dichotomique : les dirigeants indiquent $(0)$ lorsqu'ils ne recourent pas à ce système et (1) lorsqu'ils 1"utilisent.

\begin{tabular}{|c|c|c|}
\hline USEMAIL & $=$ & Envoi de messages; \\
\hline USACQDON & $=$ & Acquisition de données; \\
\hline UsPRICOM & $=$ & Prise de commandes \\
\hline USCOMINT & $=$ & Communication interne-gestion; \\
\hline USLLAIF & $=$ & Liaison avec les foumisseurs ; \\
\hline USLIVRRE & $=$ & Livraison et téception de produits ; \\
\hline USPERSON & $=$ & Personnel = ouverture sur le monde \\
\hline USOFFREP & $=$ & Offre de produits et services ; \\
\hline USREPRAG & $=$ & Repérage de nouveaux contacts; \\
\hline USECURDO & $=$ & Sécurisation de données; \\
\hline USTEVTR & $=$ & Site vitrine; \\
\hline USREGFIN & $=$ & Règlement financier. \\
\hline
\end{tabular}

\subsubsection{Le commerce électronique}

Nous avons voulu connaître les pratiques liées au commerce électronique. Nous avons demandé si les dirigeants d'entreprises font des achats sur le Web et s'ils exploitent le potentiel du Web. Les variables liées au commerce électronique sont classées selon une échelle dichotomique: la réponse à la question concernant ces systèmes est aussi dichotomique : les dirigeants indiquent $(0)$ lorsqu'ils ne recourent pas à tel système et (1) lorsqu'ils l'utilisent.

AchatWeb : $0=$ Non (ne fait pas des achats sur le Web); $1=$ Oui (fait des achats sur le Web). Webpot: $0=$ Non, (n'exploite pas le potentiel $d u$ Web); $1=$ Oui (exploite le potentiel du Web).

\subsubsection{Le matériel informatique et les logiciels}

Le matériel informatique et les logiciels sont les principales composantes du nouvel ordre technologique. Nous avons répertorié les principales composantes que les dirigeants d'entreprises utilisent davantage. 
En réponse au questionnaire, les entreprises devaient indiquer, sur une échelle de 1 à 5 , l'usage qu'elles font de chaque composante du materiel informatique et de chaque logiciel. Dans cette échelle: 1= Pas opérationnel; $2=$ En train d'être considéré, mais pas encore installé ; $3=$ En train d'être installé ; 4= Opérationnel dans certains secteurs de l'entreprise et $5=$ Opérationnel dans toute l'entreprise. Ensuite, nous avons accordé l'indice (0) si la composante n'est pas opérationnelle, si elle est en train d'être considérée, et qu'elle n'est pas encore installée ou qu'elle est en train d'être installée. Si elle est opérationnelle dans certains secteurs ou dans tous les secteurs de l'entreprise, on lui attribue un indice (1). Ainsi, on se trouve avec une division dichotomique des réponses concernant les variables suivantes relatives au matériel :

$\begin{array}{lll}\text { MTELECOP }= & \text { La télécopie; } \\ \text { MIMPLASE }= & \text { Les imprimantes laser; } \\ \text { MAUTRIMP }= & \text { Les autres imprimantes; } \\ \text { MORDINAT }= & \text { Les ordinateurs ; } \\ \text { MINIORDI }= & \text { Les mini-ordinateurs; } \\ \text { MORDINAP }= & \text { Les ordinateurs portatifs; } \\ \text { MODEMS }= & \text { Modems; } \\ \text { MCAIO }= & \text { CAIO } \\ \text { MRESOLOC }= & \text { Les réseaux locaux; } \\ \text { MTABLTRA }= & \text { Les tables traçantes; } \\ \text { MBOITEVO }= & \text { Les boites vocales; } \\ \text { MROBOT }= & \text { Les robots; } \\ \text { MLECTOPT }= & \text { Les lecteurs optiques; } \\ \text { MESSAGEL }= & \text { Les messageries électroniques; } \\ \text { MAUTRES }= & \text { Autres. }\end{array}$

Voici les variables concernant les logiciels:

$\begin{array}{lll}\text { LOGEDI } & \text { Les logiciels d'échange d'information; } \\ \text { LOGTRAT }= & \text { Les logiciels de traitement de texte; } \\ \text { LOGCOMP }= & \text { Les logiciels de comptabilité } ; \\ \text { LOGESTPR }= & \text { Les logiciels de gestion du personnel } ; \\ \text { LOGBASED }= & \text { Les logiciels de gestion de bases de données; } \\ \text { LOGCHFEL }= & \text { Les chiffriers électroniques; } \\ \text { LOGETPRO }= & \text { Les logiciels de gestion de projet } ; \\ \text { LOGSYSEX }= & \text { Les systèmes experts; } \\ \text { LOGESINV }= & \text { Les logiciels de gestion de linventaire; } \\ \text { LOGAUTRE }= & \text { Autres. }\end{array}$


A parcourir toutes les parties de ce questionnaire, on se rend compte de l'importance des variables que nous comptons mesurer dans cette recherche. Ces variables devraient pouvoir foumir des éléments indispensables pour comprendre comment les acteurs de développement se situent par rapport à l'information et à son économie, de même qu'au matériel informatique et aux logiciels. En fait, ces variables oscillent entre un cadre réservé à l'information et à son traitement et un cadre technologique dominé par des composantes relativement traditionnelles, comme le téléphone et le télécopieur, et d'autres à base d'une technologie de pointe. Cette oscillation se fait suivant le positionnement des acteurs par rapport aux divers ordres techniques et technologiques, considérés ici comme deux composantes inséparables des structures en place.

\subsection{Les orientations méthodologiques}

Les dirigeants de PME utilisent différemment les moyens et outils de travail. On peut reconnaître cet usage selon deux axes, vertical et horizontal. L'axe vertical situe les PME les unes par rapport aux autres, créant une certaine hiérarchie entre elles en fonction du degré d'importance qu'elles accordent aux anciens et aux nouveaux outils et moyens de travail, dans la gestion organisationnelle en général et dans le traitement de l'information, en particulier.

L'axe horizontal permettra de questionner nos variables mais, dans ce cas, à partir des objectifs visés par la gestion organisationnelle et le traitement de l'information. Ainsi, l'usage des outils et moyens de gestion, anciens et nouveaux, sera évalué à partir des objectifs visés par les opérations quotidiennes des entreprises : rejoindre les fournisseurs, les clients, les centres de recherche, les instances gouvernementales, etc. Précisé davantage à partir d'une analyse des nombreux moyens d'interaction qu'adoptent les dirigeants d'entreprises, cet usage permettra de saisir l'importance que l'on accorde ou non aux TIC. Cette démarche est nécessaire pour voir comment ces TIC engagent ou freinent, selon les cas, les processus d'imnovation dans chaque cadre territorial. La combinaison de ces deux perspectives, verticale et horizontale, permet de situer les dirigeants d'entreprises dans le contexte général où ils agissent.

Il faut, cependant, une observation plus poussée si nous voulons mieux inscrire les pratiques entrepreneuriales dans leurs contextes respectifs. A cette fin, des discussions et des échanges 
d'information avec certains acteurs de développement et avec des dirigeants d'entreprises fournissent des enseignements d'une grande utilité scientifique et pratique. C'est à la lumière de ces échanges et discussions que nous avons formulé la question principale de cette thèse et que nous avons interprété ce que nous avons observé sur le terrain.

\subsubsection{Position du problème}

Bien qu'en partie dictée par le parcours socio-historique d'un territoire et son insertion au sein du système national en place, la conduite de l'acteur, de l'entreprise, est soumise à des pressions, des contraintes, d'origines à la fois endogène et exogène, auxquelles il faut faire face et s'adapter. Parmi les contraintes de source endogène, il y a bien sûr l'entreprise ellemême, avec ses problèmes de financement, de main-d'œuvre, de matériel, ainsi que les nécessaires interactions quotidiennes avec son entourage. Les pressions d'origine exogène viennent, entre autres, des marchés et de la réglementation qui les entoure, de la concurrence entre firmes, territoires ou pays, etc. Pour l'acteur qui veut survivre, le territoire qui cherche à se développer, les occasions de se fourvoyer ou de se faire devancer sont nombreuses. Toutefois l'avantage habituel des uns par rapport aux autres ne parait pas inéluctable. Dorénavant existe une puissante force d'égalisation des chances de développement, depuis l'avènement de l'informatique et des TIC. II s'agit pour l'acteur, l'entreprise, le territoire, de savoir en profiter en se les appropriant et en les utilisant judicieusement.

Le présent travail se propose d'examiner comment les acteurs de développement utilisent les moyens et outils nécessaires à l'exercice de leurs fonctions et à l'atteinte de leurs objectifs. L'intérêt des acteurs pour les TIC se manifeste d'abord par la recherche puis la mise à jour continue de l'information pertinente. La décision d'adopter une technologie de pointe incite à délaisser des modes de fonctionnement et de travail dépassés et manifeste la capacité d'intégrer les vagues successives de changement. L'appropriation de la nouvelle technologie informatisation, Internet et autres - vient ensuite. Mais en définitive ce qui compte, c'est l'utilisation qu'on en fait. Là réside en effet, pour les acteurs de développement et leurs territoires, un pouvoir considérable, capable de consolider leur avantage concurrentiel et de stimuler leur progrès. L'adoption et l'utilisation des technologies nouvelles varient d'un territoire à l'autre, d'un acteur de développement à l'autre. Ce sont là des aspects importants du développement inégal des territoires, qu'il paraît utile de scruter. 


\subsubsection{La question centrale de la recherche}

Considérant que l'inégalité constatée entre les territoires réfère ordinairement aux désavantages des territoires périphériques par rapport aux territoires métropolitains, une question s'impose : Comment les territoires périphériques peuvent-ils espérer rattraper leur retard par rapport aux métropoles ? Nous posons comme hypothèse que, dans les territoires périphériques, l'appropriation et l'usage des TIC par les entreprises, pour la conduite de leurs affaires et pour leur intégration à l'économie de l'information, constituent le moyen le plus favorable à linnovation et au rattrapage de leur retard par rapport aux métropoles.

\subsubsection{Les objectifs de la recherche}

Nous visons à atteindre les objectifs suivants :

1. Connaître comment le changement technologique influence les comportements des dirigeants d'entreprises.

2. Saisir la façon dont ces dirigeants usent des composantes technologiques, particulièrement quant à l'information et à la communication.

3. Évaluer les effets de la taille des entreprises sur l'innovation.

4. Estimer le niveau d'appropriation des nouveaux systèmes informationnels par les acteurs économiques et les territoires.

5. Montrer $1^{\prime}$ inégalité des territoires par rapport à l'exploitation de l'information et de son économie.

Les variables retenues pour mener à bien cette recherche ont fait lobjet de ce chapitre. Elles ont été choisies pour leur aptitude à rendre compte de la complexité des phénomènes étudiés. C'est la nature même de notre question centrale, tirée principalement des préoccupations quotidiennes des acteurs de développement qui a dicté la démarche suivie dans ce travail. Nos constatations pourraient vraisemblablement déboucher sur des pistes d'action favorables à l'innovation. La formulation des objectifs ci-dessus est dans la ligne de ces préoccupations, à la croisée de la théorie et de la pratique. 


\section{CHAPITRE 7}

\section{LES DONNÉES RECUEILLIES}

La gestion organisationnelle, de même que les méthodes de travail, évoluent et se modifient au rythme des découvertes scientifiques et technologiques. Un peu partout, les organisations recourent à un matériel informatique sophistiqué et à des logiciels adaptés à des besoins variés. Bien que les nouveaux produits informatiques soient mis à la portée de toutes les organisations, leur appropriation varie d'une organisation à l'autre, voire d'un territoire à l'autre et cette disparité donne à la concurrence entre les organisations et entre les territoires des dimensions nouvelles. Dans tous les cas, la nouveauté est avant tout technologique. Elle affecte non seulement la gestion des organisations, mais se traduit aussi par l'émergence de nouvelles dynamiques de développement.

L'objectif de l'enquête présentée ici était de connaître comment cette nouveauté et ce développement s'opèrent dans quatre territoires du Québec: la CUQ, Saguenay, Manicouagan et Rivière-du-Loup. L'enquête a permis de mesurer le degré d'appropriation du matériel informatique et des logiciels, et d'en connaître les effets sur le traitement de l'information. 
Le questionnaire qui servit à la collecte de données comprend trois volets. Le premier concerne le profil des entreprises (nombre de places d'affaires, nombre d'employés, âge des entreprises, chiffre d'affaires et sources de concurrence).

Le deuxième volet est réservé aux besoins, à la demande et à la circulation de l'information. L'accent est mis sur les sources d'information, les moyens d'interaction, le type d'information, les activités à l'extérieur de l'organisation, la participation à des réseaux d'entreprises, les systèmes de communication et les systèmes de classement de l'information. Le troisième volet traite de l'innovation technologique. On s'y intéresse à l'usage que l'on fait du matériel informatique et d'un nombre de logiciels, de même que sur le branchement des entreprises à l'Internet et à son utilisation.

La collecte des données s'est déroulée entre juillet 2000 et janvier 2001. Notre questionnaire a été envoyé à 290 entreprises choisies de manière aléatoire, et préalablement contactées par téléphone. II faut cependant souligner que le choix des entreprises implantées au Saguenay a été fait à partir d'une liste fournie par le centre local de développement; ce qui explique pourquoi toutes les entreprises de l'échantillon de ce territoire sont affiliées à un réseau d'entreprises.

Notre questionnaire a suscité des réactions diverses, certaines entreprises de la région de Québec refusant d'y collaborer, au motif qu'elles sont trop sollicitées par les chercheurs. De plus, nous avons dû rejeter 13 cas qui présentaient des lacunes importantes empêchant de les inclure dans notre étude. Au total, notre échantillon est donc formé de 132 questionnaires, contenant des données qui se prêtent bien à l'interprétation. II s'agit d'un taux de réponse de $45,5 \%$. Nous avons reçu les derniers questionnaires fin décembre 2000 et début 2001 .

La quatrième section de ce chapitre présente une interprétation de nos données, après compilation puis traitement à l'aide du logiciel Statistical Package for the Social Science, SPSS. Ce logiciel fournit des outils statistiques qui ont servi à comparer les données portant sur les quatre territoires. Nous avons utilisé la version 10.0 pour Windows de ce logiciel. 


\subsection{Le profil des entreprises}

Les paragraphes et graphiques qui suivent montrent les caractéristiques générales des PME qui constituent notre échantillon. Les PME sont répertoriées selon leur territoire d'appartenance, le nombre de places d'affaires, le nombre d'employés, le nombre d'années d'opération, le chiffre d'affaires et les sources de concurrence.

La figure 2 indique la répartition des entreprises de notre échantillon, selon leur territoire d'implantation. On note que $10 \%$ d'entre elles sont implantées à Rivière-du-Loup et $19 \%$ à Manicouagan. La CUQ compte pour $26 \%$ et Saguenay pour $45 \%$ de l'échantillon. Ce dernier pourcentage est dû principalement à un taux de réponse plus élevé dans cette MRC.

Figure 2 : Répartition des entreprises de l'échantillon selon leur territoire d'appartenance, (en \%)

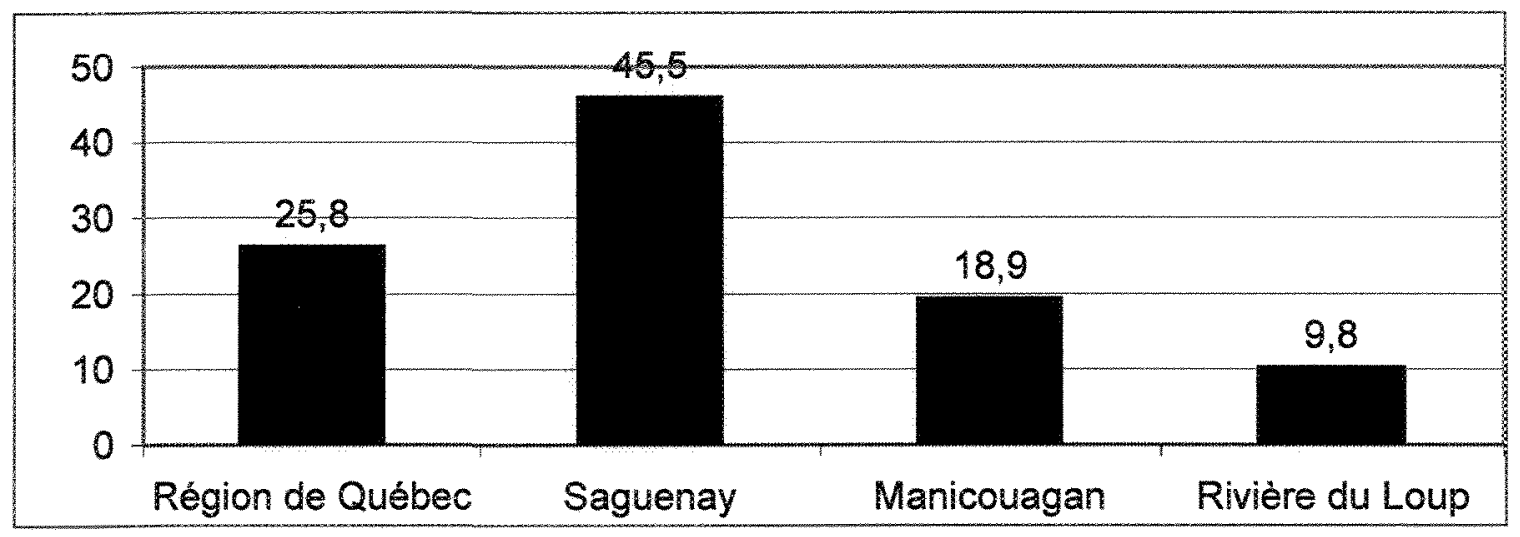

Bien que le secteur primaire soit peu présent dans notre échantillon, le tableau 9 montre des secteurs secondaire et tertiaire bien vivants et laisse croire que notre échantillon est une image raisonnablement représentative de la réalité que nous voulons analyser. Par exemple, les entreprises engagées dans l'industrie de l'information représentent $12 \%$ dans la $C U Q$, comparativement à un pourcentage allant de 4 à près de $8 \%$ dans les autres territoires. En raison de son statut urbain et de capitale, le pourcentage plus élevé dans la région de Québec 
était attendu. Il en va de même pour les services administratifs. Le tableau confirme par ailleurs ce qu'on sait de façon empirique : que dans certains territoires, à cause des ressources naturelles particulières qui s'y trouvent, une certaine spécialisation industrielle apparaît. C'est le cas, par exemple, pour la foresterie à Manicouagan, et au Saguenay pour la transformation des métaux.

Tableau 9: Les entreprises de l'échantillon selon le secteur d'activité, (en \%)

\begin{tabular}{|c|c|c|c|c|c|}
\hline & CUQ & Man. & Rivi. & Sagu. & Total \\
\hline Secteur Primaire: & 0,0 & 12 & 0,0 & 0,0 & 12,0 \\
\hline \multicolumn{6}{|l|}{ Secteur secondaire } \\
\hline Fabrication d'aliments & 0,0 & 4 & 15,4 & 8,3 & 27.7 \\
\hline Fabrication de boissons et de produits de tabac & 0,0 & 0 & 0,0 & 1,7 & 1,7 \\
\hline Fabrication de vêtements & 3,0 & 0 & 7,7 & 5,0 & 15,7 \\
\hline Fabrication de produits en bois & 0,0 & 8 & 0,0 & 6,7 & 14,7 \\
\hline Fabrication du papier & 0,0 & 4 & 15,4 & 0,0 & 19,4 \\
\hline Impression et activités connexes de soutien & 12,1 & 0 & 7,7 & 0,0 & 19,8 \\
\hline Fabrication de produits chimiques & 3,0 & 0 & 0,0 & 1.7 & 4,7 \\
\hline Fabrication de produits en caoutchouc et en plastique & 0,0 & 0 & 0,0 & 1,7 & 1,7 \\
\hline Fabrication de produits minéraux non métalliques & 6,1 & 0 & 7,7 & 5,0 & 18,8 \\
\hline Première transformation de métaux & 0,0 & 0 & 0,0 & 6,7 & 6,7 \\
\hline Fabrication de produits métalliques & 9,1 & 8 & 7,7 & 13,3 & 38,1 \\
\hline Fabrication de machines & 0,0 & 0 & 0,0 & 5,0 & 5,0 \\
\hline Fabrication de produits informatiques et électroniques & 33,3 & 0 & 0,0 & 3,3 & 36,7 \\
\hline Fabrication de matériel de transport & 3,0 & 0 & 0,0 & 3,3 & 6,4 \\
\hline Fabrication de meubles et de produits connexes & 6,1 & 12 & 7,7 & 8,3 & 34,1 \\
\hline Autres & 0,0 & 8 & 0,0 & 0,0 & 8,0 \\
\hline \multicolumn{6}{|l|}{ Secteur tertiaire } \\
\hline Transport et entreposage & 0,0 & 12 & 0,0 & 3,3 & 15,3 \\
\hline Services publics : & 0,0 & 4 & 0,0 & 0.0 & 4,0 \\
\hline Production, transport et distribution d'électricité & 0,0 & 8 & 7,7 & 1,7 & 17,4 \\
\hline Réseaux d'aqueduc, d'égout et autres & 0,0 & 8 & 7,7 & 0,0 & 15,7 \\
\hline Industrie de linformation & 12,1 & 4 & 7,7 & 6,7 & 30,5 \\
\hline Services immobiliers et services de location & 0,0 & 0 & 7,7 & 0,0 & 7,7 \\
\hline Services professionnels, scientifiques et techniques & 3,0 & 0 & 0,0 & 10,0 & 13,0 \\
\hline Services administratifs et de soutien & 6.1 & 0 & 0,0 & 1,7 & 7,7 \\
\hline Services de gestion de déchets et services d'assainissement & 0,0 & 8 & 0,0 & 3,3 & 11,3 \\
\hline Soins de santé et assistance sociale & 0,0 & 0 & 0,0 & 3,3 & 3,3 \\
\hline Hébergement et services de restauration & 3,0 & 0 & 0,0 & 0,0 & 3,0 \\
\hline Total & 100,0 & 100 & 100,0 & 100,0 & \\
\hline
\end{tabular}


Les entreprises implantées dans la CUQ et spécialisées dans la fabrication de produits informatiques et électroniques représentent $33,3 \%$; ce pourcentage est de 3,3 dans le cas du Saguenay.

Figure 3: Répartition des entreprises selon le nombre de places d'affaires, en \%

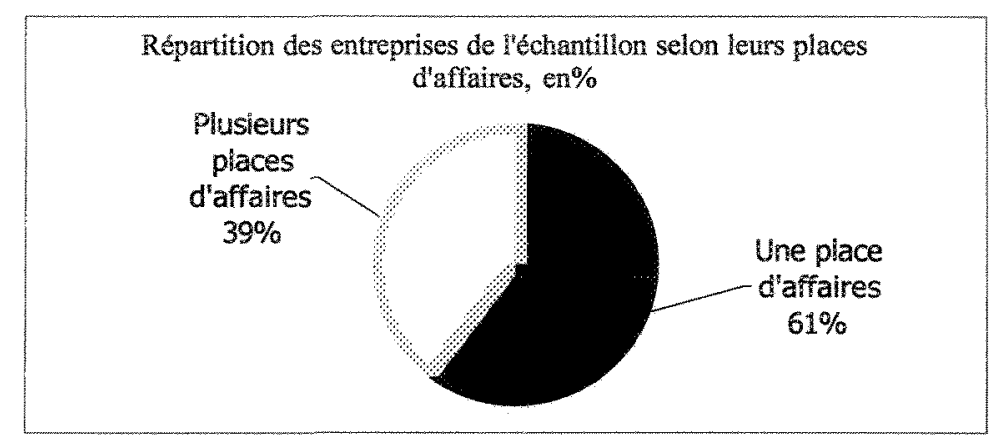

La distribution des entreprises par nombre de places d'affaires est représentée à la figure 3 . Les entreprises qui ont une seule place d'affaires représentent $61 \%$ de l'échantillon; $39 \%$ en ont plus d'une.

La distribution de l'échantillon selon le nombre d'employés est représentée à la figure 4. On remarque que $76,5 \%$ des entreprises ont 50 employés et moins, et $22 \%$ ont plus de 50 employés.

Figure 4: Répartition des entreprises selon le nombre d'employés, en \%

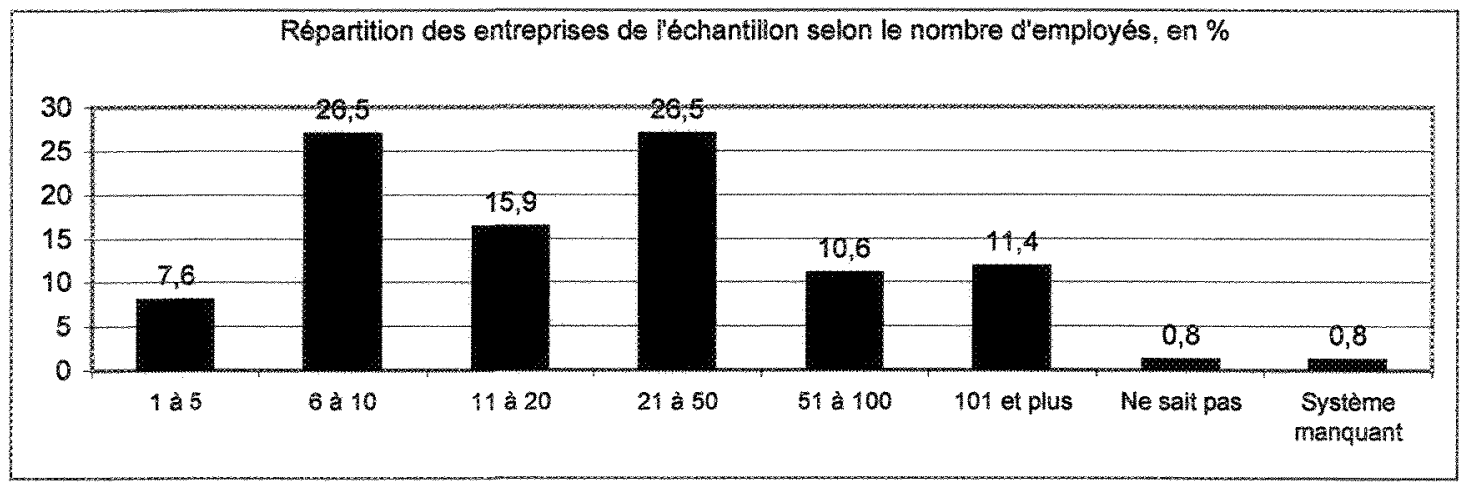


La distribution de l'échantillon selon l'âge des entreprises montre que $38 \%$ sont actives depuis moins de 10 ans, alors que $54 \%$ ont 13 ans d'activité et plus, et $36 \%$ ont plus de 16 ans d'activité. La figure 5 illustre cette répartition selon l'âge des entreprises.

Figure 5 : Répartition des entreprises selon le nombre d'années d'opération, (en \%)

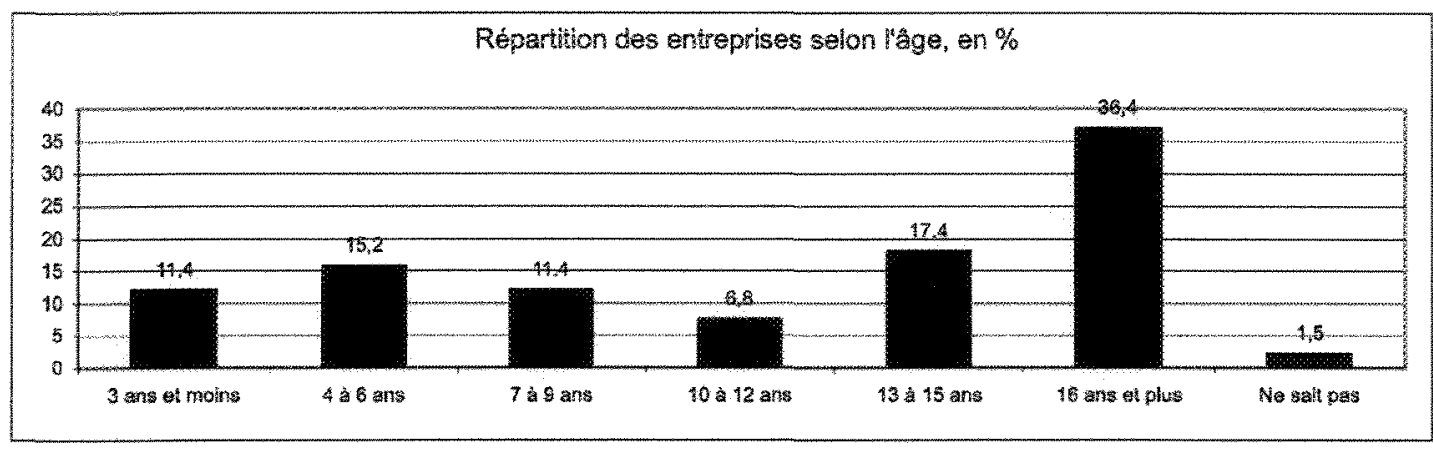

Pour $17 \%$ des entreprises, le chiffre d'affaires ne dépasse pas $500000 \$$; la plus forte proportion, soit près de $45 \%$, font entre 2 et 5 millions; et $15 \%, 5$ millions et plus.

Figure 6 : Répartition des entreprises selon le chiffre d'affaires, en $\%$

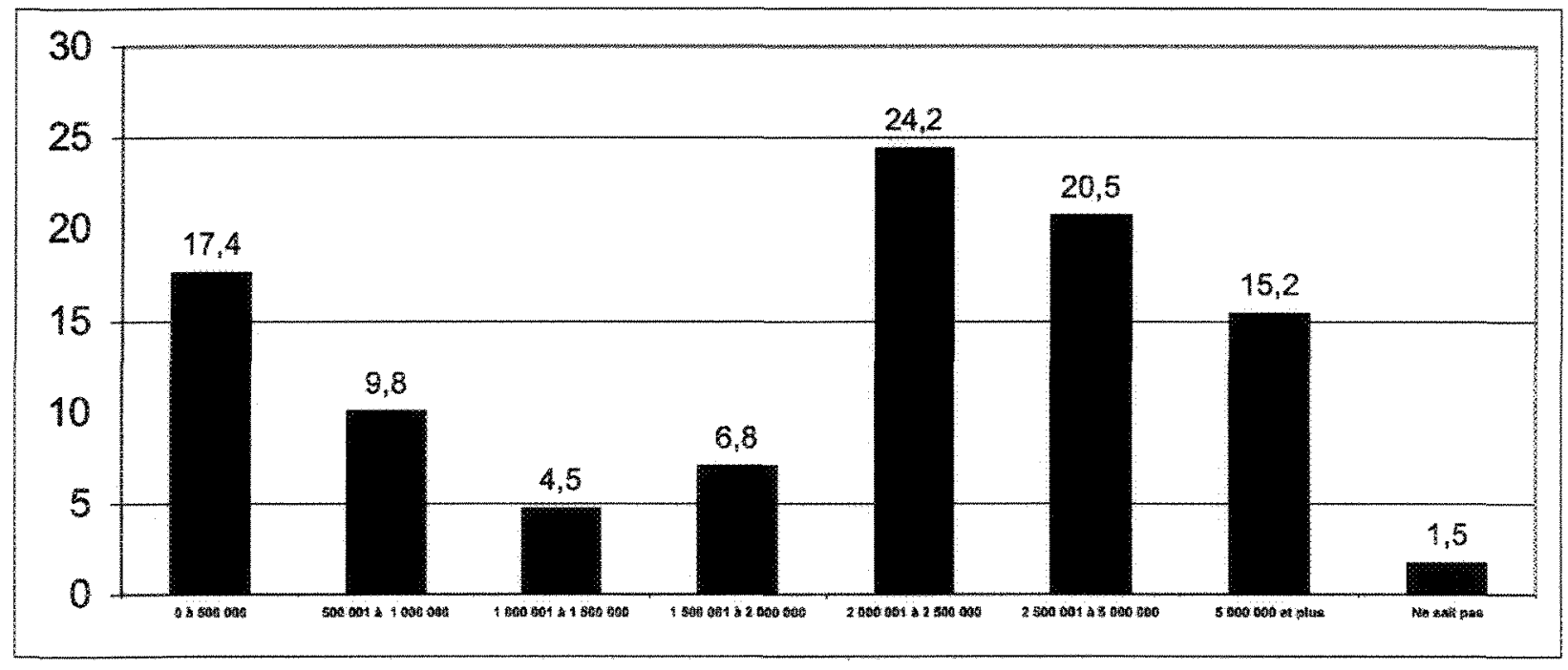

La distribution de notre échantillon selon la source de concurrence est représentée à la figure 7. On constate que $30 \%$ des entreprises mènent une concurrence locale ; $23 \%$ font face à une concurrence régionale ; $26,5 \%$ subissent une concurrence internationale et seulement 
$18 \%$ au niveau national. La même entreprise peut avoir de la concurrence à plusieurs niveaux en même temps.

Figure 7: Répartition des entreprises selon les sources de concurrence, en \%

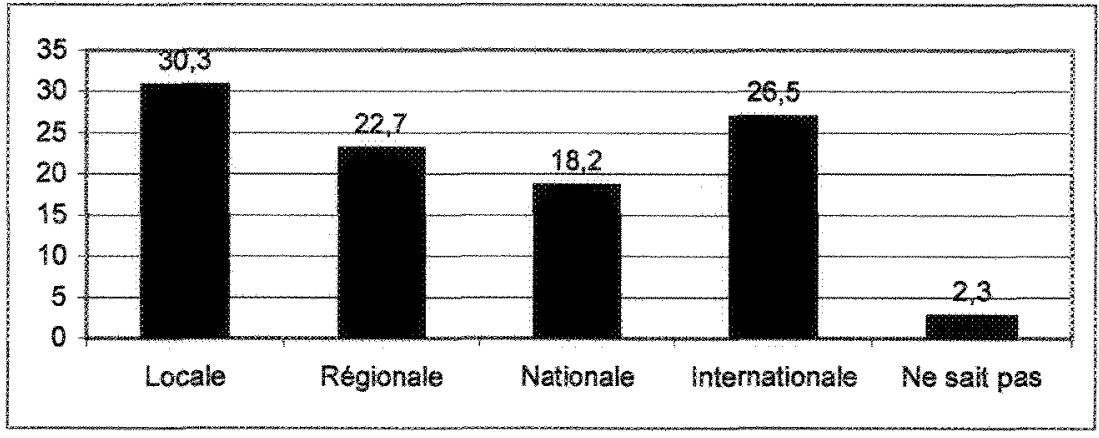

Après 1'aperçu, présenté dans un précédent chapitre, des principales caractéristiques des territoires étudiés, le tableau et les histogrammes de la présente section ont tracé le profil général de l'échantillon que nous avons tiré des entreprises de ces territoires. Les deux sections qui suivent entrent davantage dans le détail, avec la présentation des données relatives à deux domaines d'intérêt crucial pour une entreprise : l'information et les TIC.

\subsection{L'information : besoins, demande et circulation}

Cette section est consacrée aux besoins, à la demande et à la circulation de l'information. Nous avons cherché à obtenir des données sur les sources que les entrepreneurs utilisent pour recueillir l'information stratégique. Nous nous sommes également intéressé aux moyens d'interaction; aux types d'information qui intéressent les entrepreneurs et les activités auxquelles les entrepreneurs participent à l'extérieur de leurs organisations. Dans les sections suivantes, nous examinons de plus près l'ensemble de ces données.

\section{2.1. Les sources d'informations économiques}

On a cherché à connaître les principales sources d'information que les entrepreneurs utilisent dans leurs activités professionnelles, à l'aide de la question suivante: Quelles sources d'informations économiques considérez-vous les plus utiles dans vos activités professionnelles? 
Les sources utilisées très souvent sont les clients par $68 \%$, les contacts personnels $(47 \%)$ et les fournisseurs (42\%). Les sources les moins utilisées sont les centres de recherche ; $25 \%$ des entreprises ne les utilisent jamais et $34 \%$ d'entre elles ne recourent jamais aux universités. Dans notre échantillon, $23 \%$ des entreprises n'utilisent jamais les services de sociétés de développement, $28 \%$ ne sollicitent jamais de sociétés-conseils, $26,5 \%$ n'ont jamais visité d'entreprises canadiennes et $17 \%$ n'ont jamais utilisé les services fédéraux. Par ailleurs, les sources d'information atilisées souvent sont les foumisseurs, par $34 \%$ des entreprises, les centres de recherche et les sociétés-conseils, par $22 \%$; le gouvernement fédéral, par 30\%, le gouvernement provincial, par 32\%. Parmi les entreprises, $42 \%$ consultent des journaux et revues spécialisés, 33\%, des revues et journaux régionaux et un même pourcentage est rapporté dans le cas des foires et expositions.

Un nombre important d'entreprises font appel peu souvent ou rarement à des sources extérieures d'information. Ainsi, $42 \%$ des entreprises sollicitent peu les centres de recherche, $48 \%$, les universités, $40 \%$ le gouvernement fédéral, $36 \%$ le gouvernement provincial. Dans le cas de visites d'entreprises à l'étranger, il y a faible utilisation par $38 \%$ de notre échantillon; pour les sociétés-conseils, $40 \%$; pour les sociétés de développement, $45 \%$; foires et expositions, $35 \%$; visites d'entreprises canadiennes, $47 \%$. D'un autre côté, le recours à des sources d'information multiples montre une différence appréciable entre les quatre territoires. Par exemple, le pourcentage des entreprises qui recourent très souvent et souvent aux fournisseurs pour s'informer représente $38 \%$ dans la région de Québec et au Saguenay. Alors que $44 \%$ des entreprises implantées à Manicouagan s'informent très souvent auprès des fournisseurs et que $61,5 \%$ le font à Rivière-du-Loup. De même, $62 \%$ des entreprises de la région de Québec s'informent très souvent auprès des clients. Le pourcentage est de $70 \%$ au Saguenay, de $60 \%$ à Manicouagan et atteint $92 \%$ à Rivière-du-Loup. Quant aux centres de recherche et aux universités, on constate une autre réalité : $26,5 \%$ des entreprises de la région de Québec ne s'informent jamais auprès des centres de recherche et $29 \%$ auprès des universités. Ces deux pourcentages sont respectivement de $17 \%$ et de $23 \%$ au Saguenay; ils sont de $40 \%$ et de $52 \%$ à Manicouagan et de $31 \%$ et de $54 \%$ à Rivière-du-Loup.

Quoi qu'il en soit, l'ensemble de ces données montre que les sources d'information sont diversifiées d'un territoire à l'autre. II semble que les besoins d'information ne sont pas les mêmes partout, qu'ils varient en fonction des dynamiques économiques et sociales de chaque 
territoire, du genre de production et aussi de la taille des entreprises. Ainsi, les sources d'information que les entreprises utilisent le plus sont les clients, les contacts personnels, les fournisseurs et les journaux et revues spécialises; alors que les sources que ces entreprises négligent sont les centres de recherche, les universités, les sociétés-conseils, les sociétés de développement et les visites d'entreprises canadiennes. De même, les entreprises implantées à Rivière-du-Loup et à Manicouagan comptent plus sur les fournisseurs et les clients que celles implantées dans la région de Québec et au Saguenay. Enfin, les entreprises implantées à Rivière-du-Loup et à Manicouagan consultent plus les universités et les centres de recherche que celles qui sont implantées dans la région de Québec et au Saguenay.

\section{2.2. Les moyens dinteraction}

Une partie de notre enquête a été consacrée aux moyens que les entrepreneurs utilisent dans leurs interactions avec les autres organisations. La question posée était : Quels sont vos moyens d'interaction (en nombre) avec les autres organisations dans une semaine ?

La formulation de cette question est suggérée par la logique qui sous-tend l'usage que l'on fait des composantes techniques et technologiques lors des activités quotidiennes. Les réponses devaient permettre de mieux comprendre la nature de l'interdépendance entre un paradigme technologique en place et les logiques et formes organisationnelles. L'enquête s'intéressait à l'usage du téléphone, de la télécopie, des activités de face-à-face, de la place publique et du courriel. Il s'agit des moyens traditionnels (face-à-face et place publique) et d'autres modernes (le téléphone, la télécopie, le courriel).

Les questions concernent l'usage de ces moyens pour recevoir et pour émettre l'information stratégique. Les réponses montrent que le téléphone est le moyen le plus utilisé par les dirigeants : 74\% des entreprises l'utilisent pour recevoir l'information 20 fois et plus par jour et 70,5\% l'utilisent à la même fréquence pour émettre des messages. Ces deux pourcentages sont respectivement de $64 \%$ et $62 \%$ dans le cas de la télécopie et de 50 et $45,5 \%$ pour ce qui est du courriel.

De plus, la place publique est un moyen d'interaction utilisé moins de cinq fois par jour dans $69 \%$ des entreprises. De même, $20,5 \%$ de celles-ci recourent aux activités de face-à-face pour 
recevoir l'information stratégique moins de 5 fois par jour et $20 \%$ l'utilisent à la même fréquence pour émettre l'information.

Ces pourcentages, qui concernent tout notre échantillon, cachent une réalité qui varie d'un territoire à l'autre. Par exemple, $59 \%$ des entreprises de la région de Québec utilisent le téléphone plus de 20 fois par jour pour recevoir de l'information et $50 \%$ l'utilisent à la même fréquence pour l'émettre. Ces pourcentages sont respectivement de $72 \%$ et $68 \%$ à Manicouagan. Ils sont de $88 \%$ et de $86 \%$ au Saguenay et de $53,5 \%$ et de $54 \%$ à Rivière-duLoup.

Quant au rôle de la place publique pour recevoir ou émettre de l'information, $68 \%$ des entreprises de la région de Québec y recourent moins de 5 fois par jour pour en recevoir et $65 \%$ aussi peu souvent pour en diffuser. Ces deux pourcentages sont moins importants que ceux rapportés au Saguenay, soit $78 \%$ pour ces deux fonctions. Ces mêmes pourcentages sont respectivement de $60 \%$ à Manicouagan et de $46 \%$ à Rivière-du-Loup. Seulement $3 \%$ des entreprises de la région de Québec utilisent la place publique pour recevoir et émettre l'information 20 fois et plus par jour. Le pourcentage est de $8 \%$ à Manicouagan; $3 \%$ au Saguenay et $8 \%$ à Rivière-du-Loup.

Généralement, les entreprises utilisent très souvent le téléphone et la télécopie pour émettre ou recevoir l'information et on utilise moins les activités de face-à-face et la place publique. Le téléphone et la télécopie sont plus utilisés au Saguenay et à Manicouagan que dans la région de Québec et à Rivière-du-Loup.

\section{2.3. Les types d'information}

Dans l'enquête, on voulait savoir le type d'information que les dirigeants des entreprises cherchent à recueillir. La question posée était la suivante : de quel type d'informations avezvous besoin dans vos activités professionnelles ? Les résultats montrent que l'information recherchée est variable. L'information la plus recherchée concerne les nouveaux produits, la concurrence, les technologies, les techniques et les modalités de gestion. Les entreprises cherchent très souvent de l'information sur les nouveaux produits, soit à proportion de $40 \%$ de notre échantillon. Celles qui cherchent aussi très souvent l'information sur la concurrence 
représentent $31 \%$. Ce pourcentage est de $33 \%$ en ce qui concerne l'information technologique et $29,5 \%$ dans le cas de l'information technique.

Par ailleurs, $38 \%$ des entreprises de notre échantillon ne cherchent jamais d'information sur la sécurité ; $23,5 \%$ ne cherchent jamais d'information scientifique et $17 \%$ d'information juridique. L'information que l'on cherche souvent conceme la concurrence (39\%); les nouveaux produits $(47 \%)$, la technologie $(44 \%)$, la technique $(45 \%)$, les ressources publiques (31\%) et l'information sur les modalités de gestion (40\%).

Ces données varient substantiellement d'un territoire à l'autre. Ainsi, 44\% des entreprises de la région de Québec cherchent peu souvent de l'information sur la conjoncture et seulement $15 \%$ la cherchent très souvent. Les pourcentages sont respectivement de $47 \%$ et de $10 \%$ au Saguenay; ils sont de $23 \%$ et de $31 \%$ à Rivière-du-Loup. Le pourcentage des entreprises qui cherchent peu souvent cette information est de $32 \%$ à Manicouagan.

De plus, $56 \%$ des entreprises de la région de Québec cherchent très souvent de l'information sur la concurrence. Cette catégorie représente $46 \%$ à Rivière-du-Loup, 36,7\% au Saguenay et $16 \%$ à Manicouagan. Au Saguenay, 48\% des entreprises cherchent souvent cette information sur la concurrence. Ce pourcentage est comparable à celui que nous avons enregistré à Rivière-du-Loup, (46\%); mais il est un peu plus élevé comparativement à celui rapporté dans le cas de Manicouagan (28\%) et de Québec (29\%).

Certaines sources d'information sont plus sollicitées à Rivière-du-Loup, comparativement aux autres territoires. Ainsi, 54\% des entreprises de ce territoire cherchent très souvent de l'information sur les nouveaux produits. Cette catégorie représente $50 \%$ dans la région de Québec et $40 \%$ au Saguenay. Ce même pourcentage ne dépasse pas $20 \%$ à Manicouagan. De plus, $31 \%$ des entreprises de la région de Rivière-du-Loup cherchent très souvent de l'information sur les exportations. Ce pourcentage est de $27 \%$ au Saguenay et de $12 \%$ dans la région de Québec.

En général, les entreprises cherchent davantage l'information qui concerne les nouveaux produits, la concurrence, l'information technologique, technique et celle relative aux modalités de gestion. Les entreprises s'intéressent moins à l'information concernant la 
sécurité, l'information scientifique et l'information juridique. Comparativement aux entreprises implantées à Manicouagan et à Rivière-du-Loup, les entreprises de Québec et du Saguenay cherchent moins l'information sur la conjoncture, la concurrence. De même, les entreprises implantées à Rivière-du-Loup et Québec s'intéressent davantage à l'information concernant les nouveaux produits, les exportations.

\section{2.4. Les activités à l'extérieur}

La question suivante concemait les activités en dehors de l'entreprise : Participez-vous à des activités à l'extérieur de votre milieu de travail ? Les résultats montrent que les dirigeants de ces entreprises participent à de nombreuses activités extérieures. Les repas sont une occasion préférée par un grand nombre : $23,5 \%$ d'entre eux y participent très souvent; $38 \%$ y participent souvent; $29,5 \%$ y participent peu souvent et seulement $7 \%$ n'y participent jamais. Dans le cas des expositions, ces mêmes pourcentages sont respectivement de $8 \%$, $36 \%, 48 \%$ et $8 \%$.

Par ailleurs, $38 \%$ de ces dirigeants ne participent jamais aux activités caritatives ; $36 \%$ y participent peu souvent. Ces deux pourcentages sont respectivement de $23,5 \%$ et $51,5 \%$ dans le cas des événements mondains. Ces mêmes pourcentages sont de $17 \%$ et de $49 \%$ pour ce qui est des événements spéciaux. Ils sont aussi, respectivement, de $23,5 \%$ et de $4 \%$ dans le cas des activités de reconnaissance et de $35 \%$ et de $45 \%$ dans le cas des activités liées à l'environnement.

Si ces données montrent que le pourcentage des entreprises qui participent à diverses activités extérieures varie selon le type d'activités, elles cachent cependant des écarts significatifs entre les territoires, en ce qui concerne chacune de ces activités.

Pour ce qui est des activités caritatives, un fort pourcentage n'y participe jamais, $44 \%$ dans la région de Québec, 46\% à Rivière-du-Loup, $33 \%$ au Saguenay et $36 \%$ à Manicouagan. Par ailleurs, $28 \%$ des entreprises du Saguenay participent très souvent à des repas. Ce pourcentage est de 26,5\% dans la région de Québec et de 20\% à Manicouagan. Ceux qui y participent souvent représentent $54 \%$ à Rivière-du-Loup; $38 \%$ au Saguenay; $36 \%$ à Manicouagan et $32 \%$ dans la région de Québec. En général, les données montrent que les 
dirigeants des entreprises participent inégalement aux différentes activités organisées à l'extérieur de leur organisation.

Les dirigeants d'entreprises s'intéressent principalement aux repas, aux activités caritatives et aux activités de reconnaissance. Un pourcentage assez important des dirigeants s'intéresse moins aux expositions et voit peu d'utilité aux activités caritatives. Comparativement avec Québec et Saguenay, les dirigeants des entreprises implantées à Manicouagan et à Rivière-duLoup participent davantage aux "Repas".

\section{2.5. L'affiliation à des réseaux d'entreprises}

Une question importante portait sur l'affiliation des entreprises à des réseaux interorganisationnels. La question était la suivante : Votre entreprise participe-t-elle à des réseaux d'entreprises (trois entreprises ou plus) ? Dans ces réseaux, des directeurs, des administrateurs ou les entreprises elles-mêmes sont les principaux acteurs considérés. On cherche donc à comprendre si ces acteurs s'impliquent dans des rapports plus formels de collaboration. Ordinairement, cette implication facilite l'acquisition d'information stratégique et stimule l'échange de certains services. Elle permet à ces mêmes acteurs de mieux se préparer collectivement à affronter les imprévus de conjonctures changeantes.

En général, les réseaux inter-organisationnels se distinguent par des rapports de coordination et de coopération, mais aussi de concurrence. L'échange d'information, de savoir et de savoirfaire constitue de la sorte une plate-forme commune, favorable à une prise en charge collective et à la mise en place d'un ensemble de standards, adaptés aux besoins du développement local comme à ceux du marché.

Dans la pratique, la réponse à cette question a permis de constater que $36 \%$ des entreprises participent à des réseaux inter-organisationnels et $62 \%$ n'y participent pas.

Ces résultats sont représentés dans la figure 8 . On y voit que ces pourcentages masquent les réalités fort différentes des quatre territoires. En effet, $41 \%$ des entreprises de la région de Québec participent à ces réseaux. Ce pourcentage est plus élevé comparativement à celui enregistré à Manicouagan (24\%) et à Rivière-du-Loup (15\%), mais inférieur à celui du Saguenay, où toutes les entreprises de l'échantillon sont affiliées. 
Figure 8: L'affiliation des entreprises à des réseaux

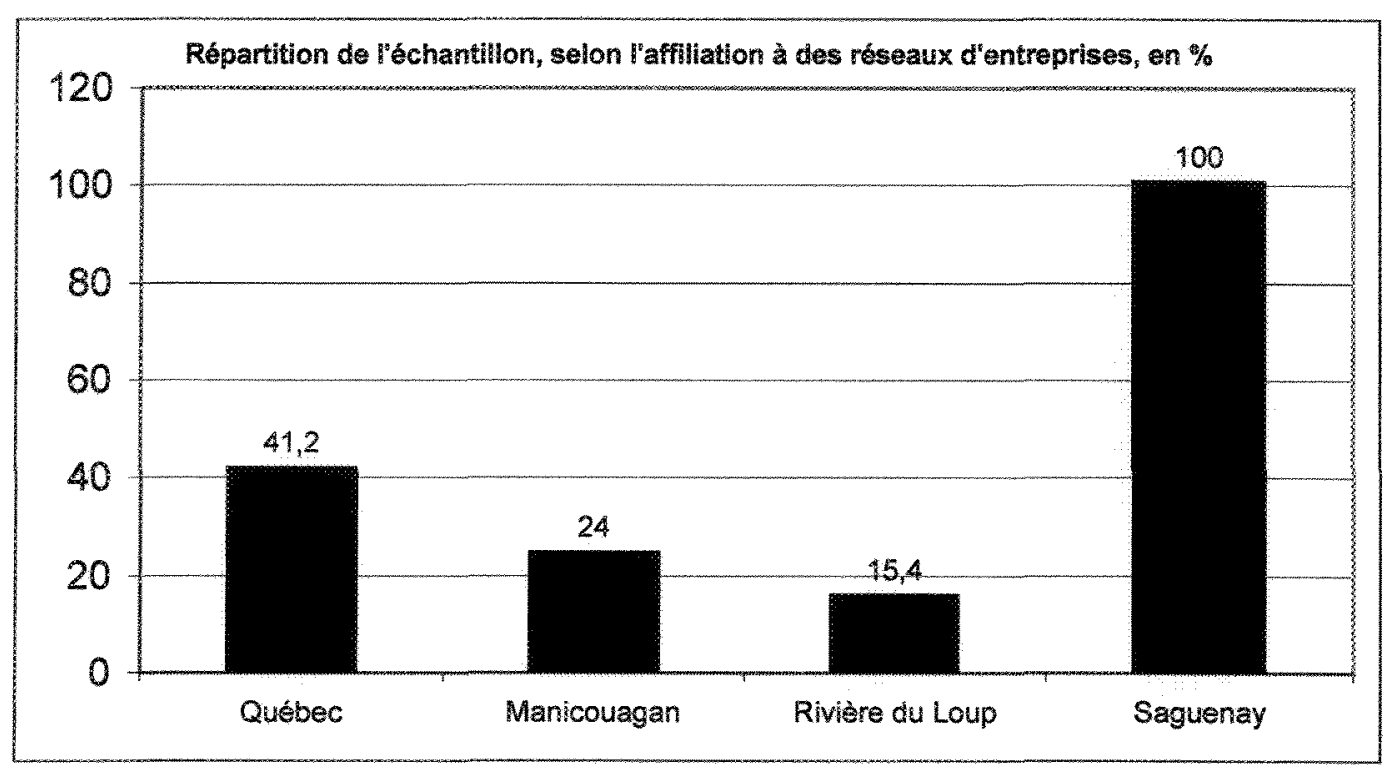

7.2.6. Les systèmes de communication interne

Les systèmes de communication constituent un moyen par lequel les dirigeants d'entreprises facilitent la circulation de l'information à l'intérieur de leurs organisations. Souvent, on utilise ces systèmes pour rejoindre l'ensemble du personnel. Chaque organisation choisit un ou plusieurs systèmes mieux adaptés à ses besoins. À cet égard, nous avons posé la question suivante : Avez-vous un système de communication à l'intérieur de l'entreprise ? La figure 9 illustre les résultats obtenus.

Les entreprises de notre échantillon adoptent divers systèmes de communication. Les réunions du personnel constituent le système le plus utilisé : $72 \%$ de ces entreprises y recourent pour véhiculer de l'information à l'interne. Les entreprises qui comptent sur les tableaux de nouvelles à cette fin $: 36 \%$. Un examen plus approfondi montre une variation appréciable entre les territoires. Par exemple, 35\% des entreprises implantées au Saguenay utilisent le bulletin interne comme système de communication, contre seulement $15,4 \%$ à Rivière-duLoup ; $28 \%$ à Manicouagan et $26,5 \%$ dans la région de Québec. 


\section{Figure 9: Les systèmes de communication adoptés par les entreprises, tout l'échantillon}

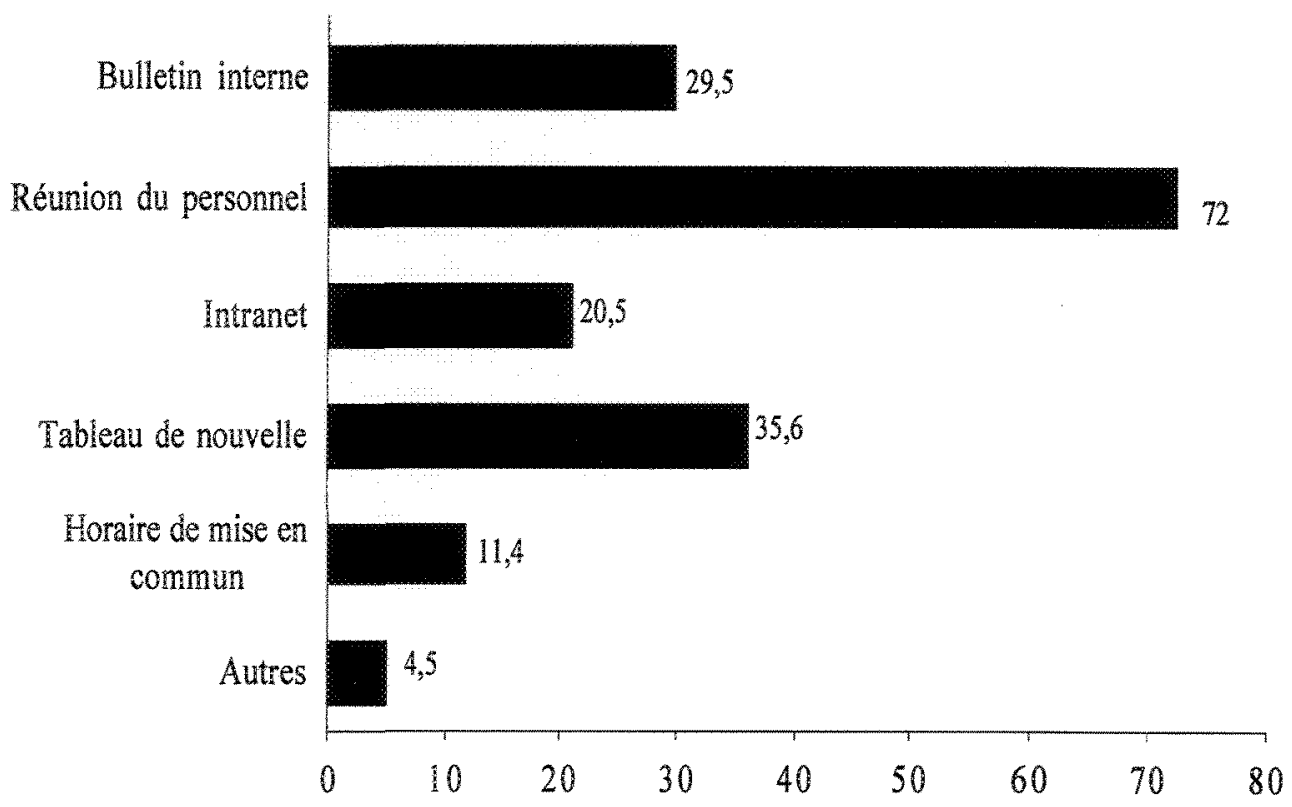

De plus, les entreprises utilisent le tableau de nouvelles à proportion de $26,5 \%$ dans la région de Québec; elles représentent 24\% à Manicouagan; $15 \%$ à Rivière-du-Loup et $30 \%$ au Saguenay. Dans la région de Québec, $35 \%$ de ces entreprises utilisent l'Intranet comme système interne de communication. Ce pourcentage est de $20 \%$ au Saguenay, alors qu'il ne dépasse pas $8 \%$ à Manicouagan et à Rivière-du-Loup. II faut souligner cependant qu'une entreprise peut utiliser plusieurs systèmes de communication à la fois. Il semble que les moyens de communication interne choisis dépendent principalement de la taille de l'entreprise.

Les entreprises préfèrent les réunions du personnel, les tableaux de nouvelles et les bulletins internes. Un pourcentage important de dirigeants d'entreprises recourent à l'Intranet. On utilise l'Intranet davantage dans la région de Québec que dans les autres territoires.

\subsubsection{Le classement de l'information}

L'avènement des nouvelles technologies impose de nouvelles façons de gérer les organisations. Des modifications des pratiques accoutumées deviennent nécessaires, et chaque organisation adopte un ou plusieurs systèmes de classement de l'information. Par rapport aux anciens, les nouveaux systèmes permettent une grande flexibilité mais en même temps 
commandent de bien considérer la finalité de l'information conservée. Le choix d'un système de classement de l'information constitue un indicateur de la capacité de l'entreprise à s'adapter aux vagues de changement apportées par les nouvelles technologies. Le questionnaire portait donc sur les différents systèmes de classement de l'information. La question était la suivante : De quelle façon classez-vous l'information reçue dans l'entreprise ? La figure 10 représente les réponses obtenues.

Figure 10: Le classement de l'information, tout l'échantillon, en \%

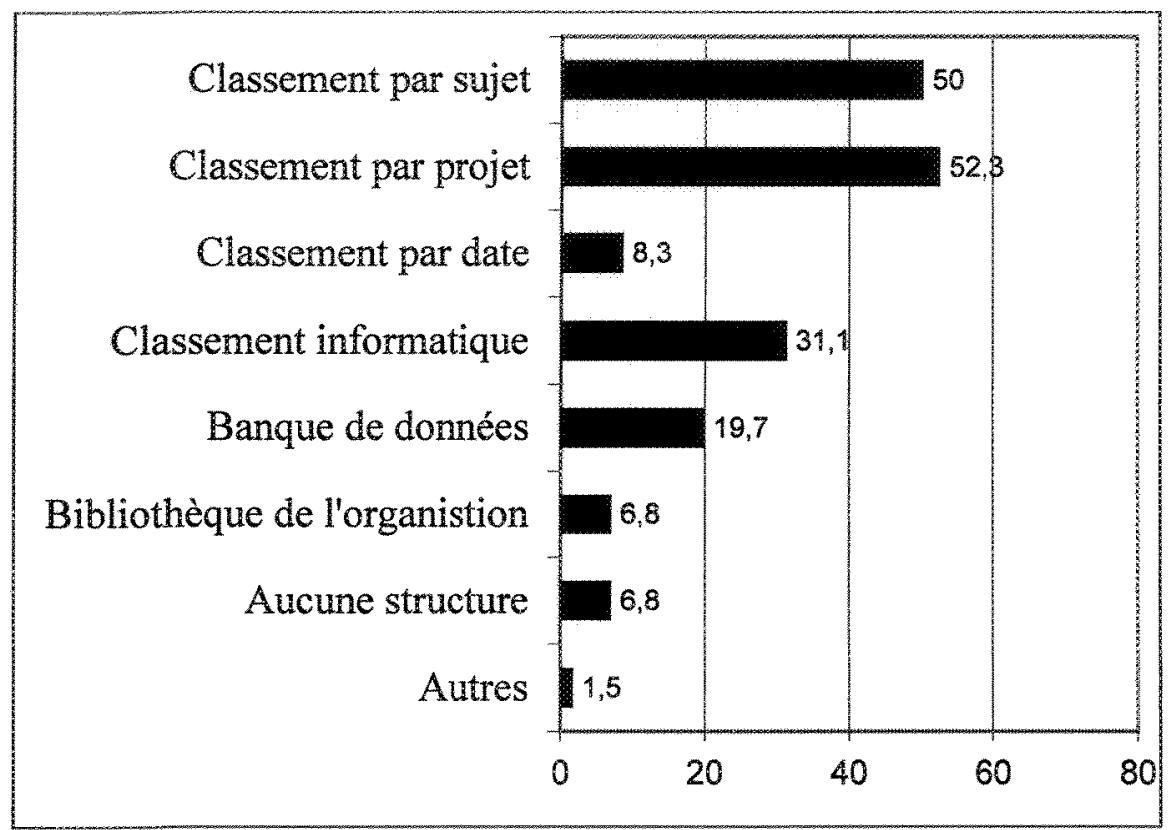

Le classement par sujet est adopté par $49 \%$ des entreprises de notre échantillon. A Manicouagan, le taux est de $55 \% ; 48 \%$ dans la région de Québec, 46\% à Rivière-du-Loup, $32 \%$ au Saguenay. De même, 39\% des entreprises de notre échantillon optent pour le classement par projet. Ce pourcentage est de $53 \%$ dans la région de Québec; de $68 \%$ à Manicouagan ; $43 \%$ à Rivière-du-Loup et 38,5\% au Saguenay.

Le classement de l'information par date est adopté par seulement $8 \%$ des entreprises de notre échantillon. Cette catégorie représente $6 \%$ dans la région de Québec. Le pourcentage est un peu plus élevé à Manicouagan, où il atteint $12 \%$, alors qu'il ne dépasse pas $7 \%$ à Rivière-duLoup. 
Quant au classement informatique de l'information, il est utilisé par $44 \%$ des entreprises de la région de Québec. Ce pourcentage dépasse ceux relevés au Saguenay (27\%), à Rivière-duLoup (23\%) et à Manicouagan (28\%). C'est aussi dans la région de Québec que l'on a enregistré le pourcentage le plus élevé quant à l'utilisation des banques de données ; $32 \%$ des entreprises de cette région utilisent ces banques, contre $16 \%$ à Manicouagan, $17 \%$ au Saguenay et seulement $8 \%$ à Rivière-du-Loup.

Les entreprises préfèrent le classement par sujet et par projet ; et on constate la montée du classement informatique et des banques de données. Le classement par sujet et par projet est plus important dans la région de Québec et au Saguenay comparativement à Rivière-du-Loup et à Manicouagan. Comparativement aux autres territoires, c'est dans la région de Québec qu'un pourcentage important d'entreprises recourent au classement informatique de l'information et aux banques de données.

\subsection{L'appropriation de la nouvelle technologie}

L'appropriation technologique peut être mesurée à partir de plusieurs indicateurs. Dans le cadre de cette enquête, le choix a été fixé sur le branchement des entreprises à l'Internet, l'utilisation de l'Internet, l'usage du Web, le soutien extérieur, et l'usage du matériel informatique et des logiciels. Ces indicateurs montrent à quel degré les entreprises s'approprient les nouvelles technologies et comment ces technologies modifient les pratiques entrepreneuriales.

\section{3.1. Le branchement des entreprises à Internet}

On ne peut guère aborder l'innovation technologique sans parler du branchement à l'Internet. Si ce branchement est un indicateur important de cette innovation, il est cependant difficile d'évaluer comment il contribue à transformer le monde des affaires. Un moyen utile pour illustrer cette innovation est la date de branchement des acteurs à l'Internet. La référence à cette date est susceptible d'aider à établir une certaine hiérarchie entre les quatre territoires retenus pour notre analyse. 
On posait la question suivante : Depuis combien de temps êtes-vous branché à Internet ? La figure 11 représente les résultats obtenus. Elle montre que 51,5\% des entreprises de notre échantillon sont branchées à l'Internet depuis plus de deux ans. La région de Québec vient en tête, (82\%) suivie de Saguenay (43\%), Manicouagan (40\%) et Rivière-du-Loup (31\%). On apprend aussi que $6 \%$ des entreprises de notre échantillon ne sont pas encore branchées à 1'Internet. À Rivière-du-Loup, cette catégorie représente $23 \%$ des entreprises, contre seulement $5 \%$ au Saguenay et $8 \%$ à Manicouagan.

Figure 11: Le branchement des entreprises à I'Internet

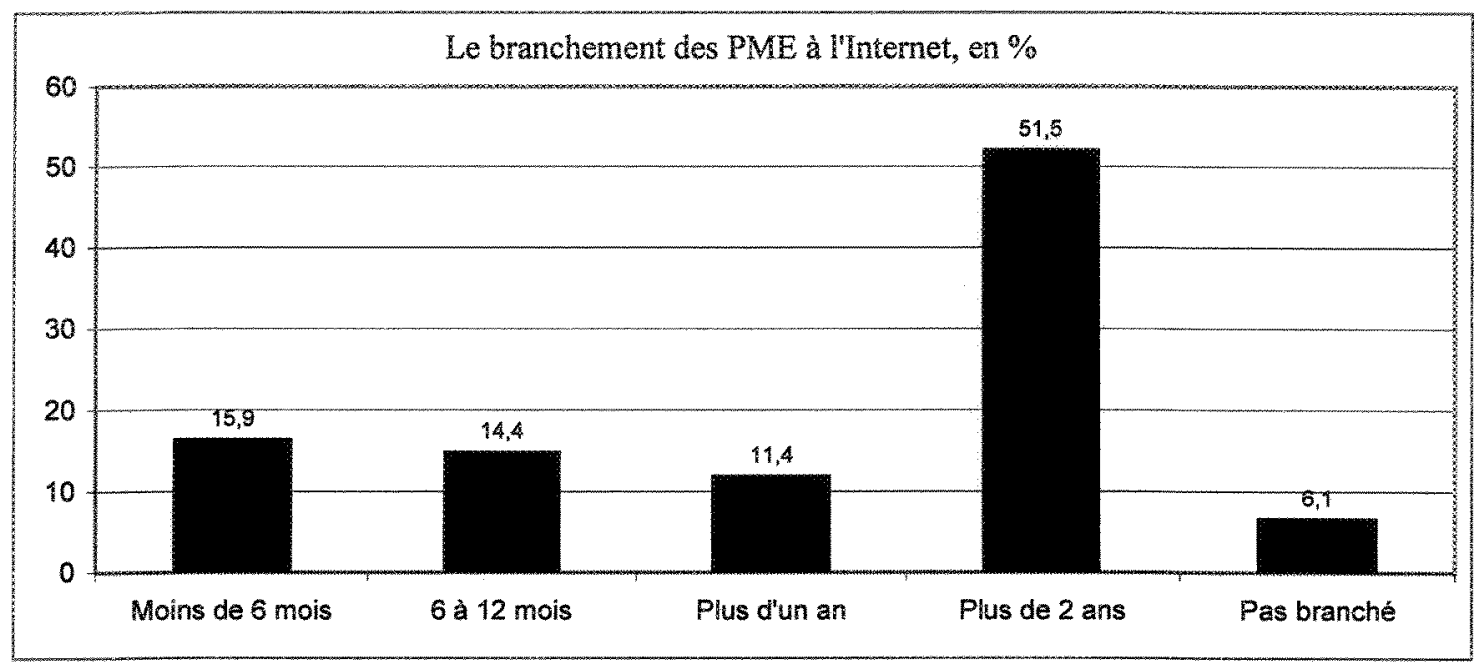

\section{3.2. L'utilisation d'Internet}

Le taux d'utilisation d'Internet par les entreprises constitue un autre indicateur de l'innovation technologique. Il faut donc chercher à comprendre comment cet usage se manifeste dans les différents territoires. La question était la suivante: Quelles sont les utilisations que vous effectuez à l'aide d'Internet ? La figure 12 représente les résultats obtenus.

L'Internet sert à $86 \%$ des entreprises de notre échantillon pour l'envoi de courriel. Au Saguenay et à Québec, ce pourcentage est très élevé ; il y atteint respectivement $97 \%$ et $91 \%$. Le pourcentage est de $88 \%$ à Manicouagan et de $77 \%$ à Rivière-du-Loup.

On peut donc dire que le recours au courriel est l'une des principales tâches que ces organisations réalisent par Internet. De même, $67 \%$ utilisent Internet pour acquérir des 
données stratégiques. Le pourcentage est de 73,5\% dans la région de Québec et de $73 \%$ au Saguenay. Il est relativement faible à Manicouagan (52\%) et à Rivière-du-Loup (46\%).

Les entreprises des quatre territoires utilisent d'abord Internet pour envoyer des courriels. Elles l'utilisent aussi pour acquérir des données, mais cette catégorie est plus importante dans la région de Québec et au Saguenay qu'à Manicouagan et à Rivière-du-Loup. Les entreprises implantées dans la région de Québec et au Saguenay utilisent Internet davantage pour établir des liaisons avec les foumisseurs, livrer et recevoir des produits et à des fins personnelles.

À Manicouagan, 20\% des entreprises utilisent l'Internet pour les prises de commandes. Ce pourcentage est de $23 \%$ à Rivière-du-Loup, 30\% au Saguenay et atteint $47 \%$ dans la CUQ. L'ensemble de ces résultats montre aussi que $65 \%$ des entreprises de la région de Québec profitent de l'Internet pour établir des liaisons avec les fournisseurs, $41 \%$ s'en servent pour livrer et recevoir des produits et $47 \%$ des dirigeants de ces organisations l'utilisent à des fins personnelles et d'ouverture sur le monde. Le Saguenay vient en deuxième place, avec des pourcentages qui sont respectivement de $52 \% ; 25 \%$ et $20 \%$.

Figure 12: L'usage de l'Internet, tout l'échantillon, en $\%$

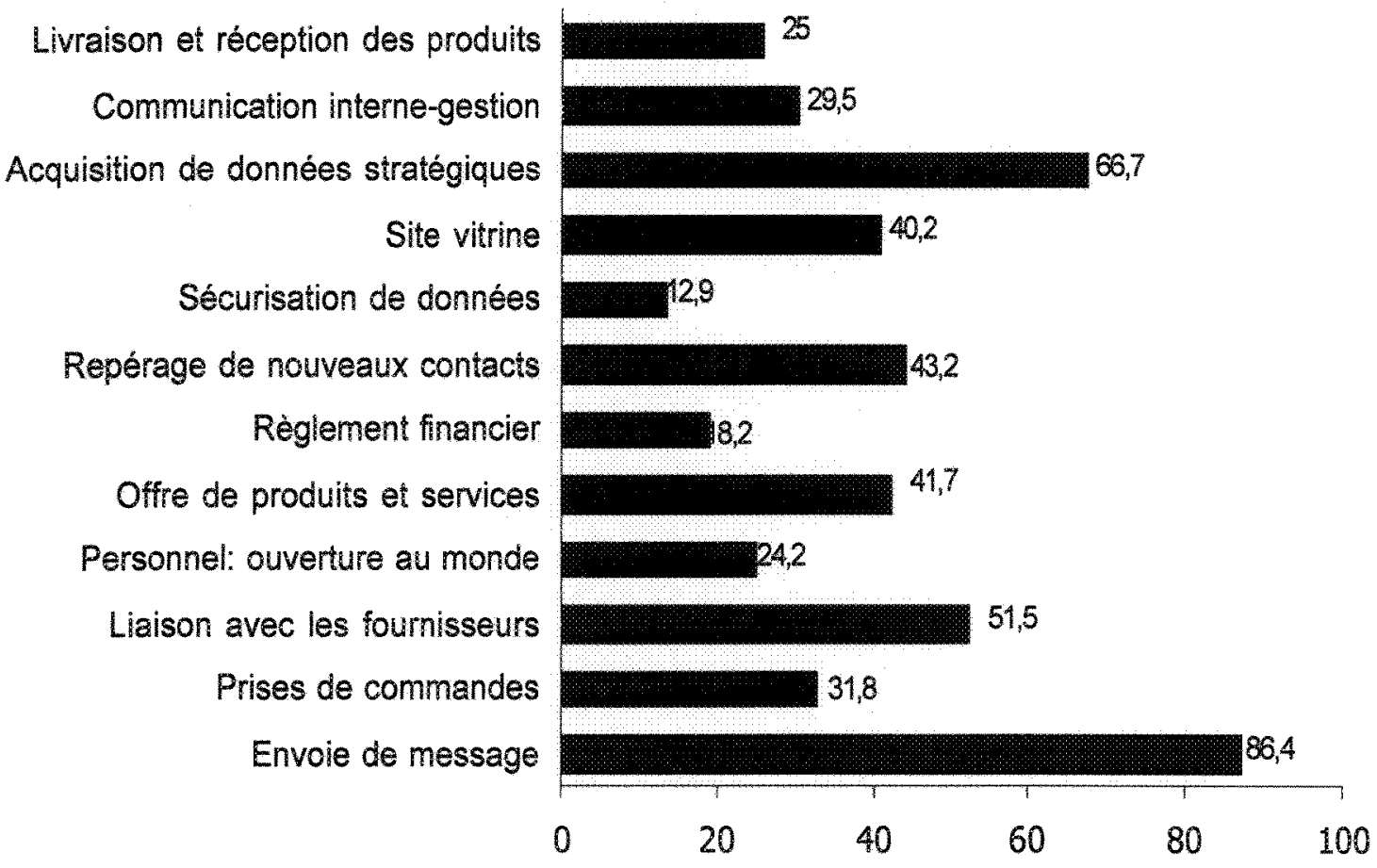




\section{3.3. L'usage du Web et de son potentiel}

La troisième question posée dans le cadre de cette section consacrée à l'innovation technologique porte sur l'usage du Web dans le domaine commercial. Plus précisément, on voulait savoir si les entreprises faisaient des achats sur le Web et à quel point elles utilisent son potentiel. On posait les deux questions suivantes : Faites-vous des achats sur le Web? Considérez-vous que vous utilisez suffisamment le potentiel du commerce électronique? Dans la figure 13, nous représentons les résultats obtenus. On constate que $32 \%$ des entreprises de notre échantillon font des achats sur le Web. Le pourcentage est plus élevé dans la région de Québec ( $50 \%$ ). Il ne dépasse pas $33 \%$ au Saguenay et s'établit à $15 \%$ à Rivièredu-Loup et à seulement $12 \%$ à Manicouagan. Par ailleurs, seulement $20 \%$ des entreprises affirment exploiter suffisamment le potentiel du Web. Ce pourcentage est de $41 \%$ dans la région de Québec ; $16 \%$ à Manicouagan ; $13 \%$ au Saguenay. Ce sont surtout les entreprises implantées dans la région de Québec et au Saguenay qui utilisent le Web pour faire des achats. À l'exception de Québec, les entreprises des autres territoires sont peu nombreuses à exploiter comme elles le pourraient les possibilités du Web.

Figure 13: L'achat et l'exploitation du potentiel du Web, en \%

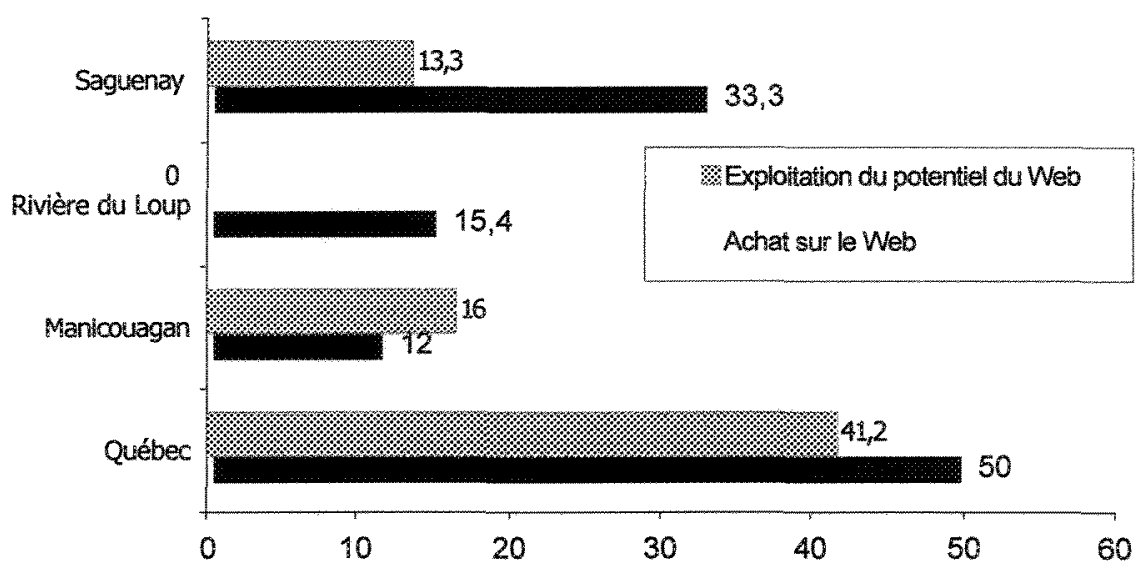




\section{3.4. Le soutien extérieur}

Pour plusieurs entreprises, l'innovation technologique exige des compétences qui ne sont pas toujours à leur portée. Souvent, elles cherchent à combler le manque d'une main-d'œuvre qualifiée par le recours à des ressources extérieures. Nous avons essayé d'identifier la nature du soutien extérieur recherché par les entreprises implantées dans les quatre territoires retenus pour notre analyse. La question était la suivante : à quels soutiens extérieurs l'entreprise peutelle recourir pour des questions reliées aux technologies de l'information?

Les réponses recueillies ont été utilisées pour construire la figure 14 . On constate que $52 \%$ des entreprises de notre échantillon utilisent les services offerts par les conselllers et les fournisseurs en matériel et logiciels; $29 \%$ profitent des services offerts par les fournisseurs d'accès à l'Internet; $22 \%$ recourent aux services que proposent les créateurs de pages Web.

Les entreprises des quatre territoires sollicitent principalement les services des conseillers et fournisseurs en matériel informatique et logiciels et des fournisseurs d'accès à Internet. Il n'y a pas de différence significative entre les quatre territoires.

Figure 14: Le soutien extérieur recherché par les entreprises, en \%

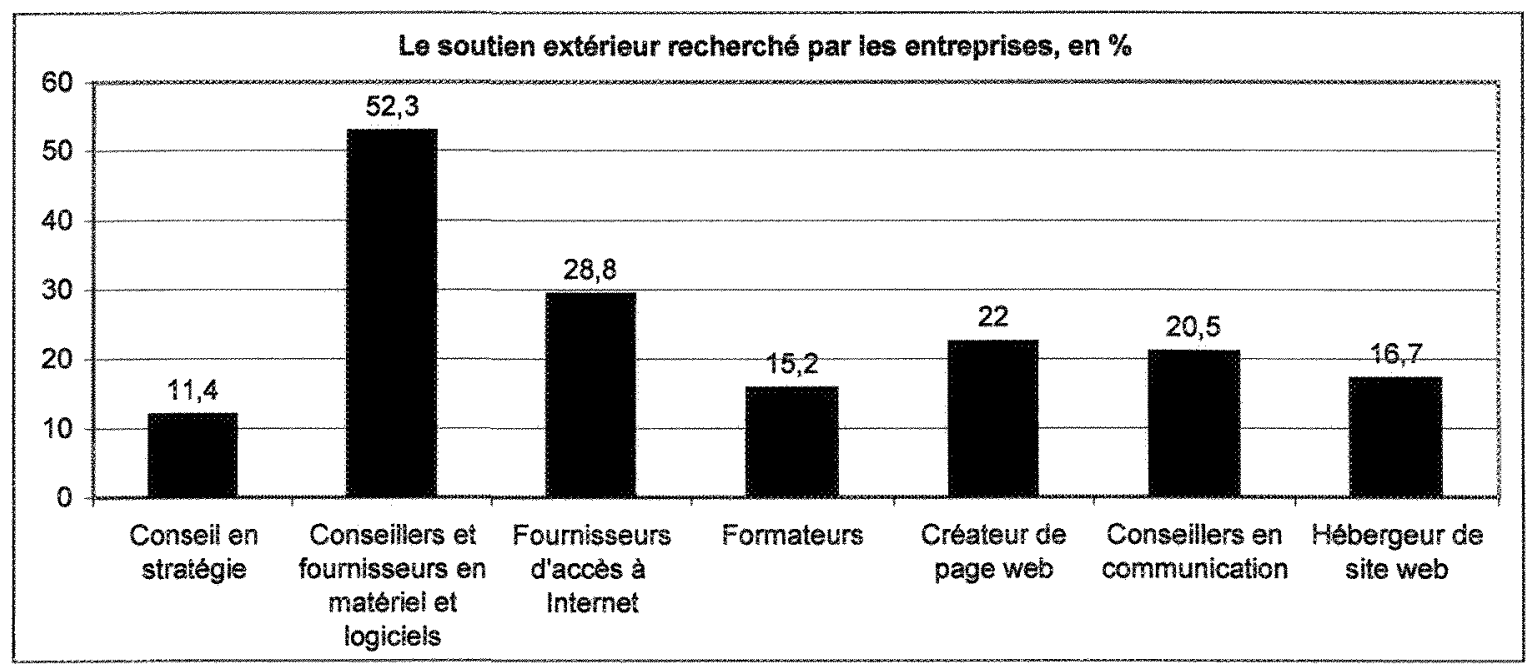




\subsubsection{Le matériel informatique et les logiciels}

L'usage du matériel informatique et d'un nombre de logiciels est un autre indicateur important de l'innovation technologique. Les composantes informatiques sont nombreuses, variées et servent à de nombreuses tâches liées à la gestion des organisations. Le matériel et les logiciels se développent rapidement, incitant l'organisation à s'efforcer de mieux s'adapter aux changements qui en résultent. Généralement, les résultats sont considérables, surtout quand les dirigeants ont choisi le matériel et les logiciels qui répondent adéquatement aux besoins ressentis à l'intérieur de l'organisation.

Cependant, le développement du secteur informatique et des logiciels est tellement rapide que plusieurs entreprises ne parviennent pas à suivre les vagues de changement. Il y a donc intérêt pour notre enquête de chercher à comprendre comment ces changements modifient les pratiques entrepreneuriales et agissent sur la société. En effet, l'usage de l'informatique influence la prise de décision au sein de toute organisation.

Les composantes technologiques qui nous intéressent se placent donc au centre même des décisions et des actions entrepreneuriales. C'est pourquoi la question posée a été formulée en ces termes: Quel est le niveau de pénétration de chacune des technologies de l'information dans votre entreprise?

On constate que diverses composantes du matériel informatique sont opérationnelles presque partout : le télécopieur, l'ordinateur et les imprimantes. Le télécopieur est utilisé dans $95 \%$ des entreprises. Le pourcentage est de $92 \%$ dans le cas des ordinateurs. De même, les imprimantes sont utilisées dans $96 \%$ des entreprises de notre échantillon. Par ailleurs, le pourcentage des entreprises qui n'utilisent pas de télécopieur et d'autres imprimantes ne dépasse pas 3\%. Dans le cas des ordinateurs, ce pourcentage est de 6\%. D'un autre côté, les composantes de l'équipement informatique qui sont moins utilisées sont les tables traçantes, qui n'existent pas dans $61 \%$ des entreprises québécoises; CAIO (37\%); lecteur optique $(55 \%)$ et les robots $(69 \%)$. 
Dans un deuxième temps, nous nous sommes intéressé aux logiciels atilisés par les entreprises. Les logiciels de traitement de texte sont utilisés dans $92 \%$ des entreprises de l'échantillon. Ce pourcentage est de $93 \%$ dans le cas des logiciels de comptabilité. Il est de $78 \%$ pour ce qui est des logiciels de traitement de bases de données. Les logiciels de gestion d'inventaire sont utilisés dans $58 \%$ des cas. Par ailleurs, $53 \%$ des entreprises n'ont pas de logiciels servant de système expert. De même, il y a absence de logiciels d'échange d'information informatisée dans $50 \%$ des cas. Quant aux logiciels de gestion du personnel, ils sont absents chez $35 \%$ des entreprises; le taux est de $14 \%$ en ce qui concerne les logiciels de gestion des bases de données.

Afin de mieux comprendre l'ampleur des transformations qu'apporte l'usage des nouvelles technologies dans les entreprises québécoises, nous avons tenté de mesurer plusieurs indicateurs. Les résultats sont présentés selon les méthodes statistiques descriptives. Les données sont comparées en fréquence et en pourcentage. Ces résultats et données confirment que les entreprises, quelle que soit leur région d'implantation, s'adaptent de manière inégale à l'avènement et au développement des technologies. Les résultats que nous présentons ici montrent que la disparité entre les territoires prend des dimensions nouvelles; une étude subséquente serait sans doute utile à qui veut saisir l'ampleur des changements occasionnés par l'innovation technologique.

Les entreprises s'approprient surtout les télécopieurs, les ordinateurs et les autres imprimantes. Elle s'approprient moins le matériel qui exige un très haut degré de spécialisation : (CAIO, lecteur optique et les robots). Il n'y a aucune différence à cet égard entre les quatre territoires. Les entreprises s'approprient beaucoup les logiciels de base (traitement de texte, traitement de données, etc.) mais s'approprient moins les logiciels plus sophistiqués. À cet égard, il n'y a pas de différence significative entre les quatre territoires.

\subsection{L'enseignement des données recueillies}

Les données collectées au cours de l'enquête ont été traitées en vue de leur interprétation et d'une analyse comparative. C'est l'objet de cette section. Le type d'analyse choisie est d'abord décrit. Vient ensuite l'analyse proprement dite, où les quatre territoires choisis pour cette étude sont comparés, les résultats consignés en tableau et succinctement interprétés. 
Afin d'obtenir une comparaison équitable de nos quatre territoires, il simposait de systématiser ef de pondérer les données recueillies au cours de notre enquête. Ces données, réparties en dix thèmes principaux (sources d'information, types d'information, classements d'information, etc.) sont représentatives, pour les fins du présent travail, du comportement des entreprises. Comme on verra aux tableaux qui suivent, chacun des thèmes est évalué selon plusieurs variables. Et pour chacun des thèmes, les quatre territoires sont comparés, d'après les valeurs attribuées aux variables congruentes.

Pour comparer et classer les territoires avec objectivité, et produire une analyse significative, il fallait donc créer des indices. Chaque indice utilisé est calculé à partir de trois groupes de données, formant chacun un sous-indice :

- les scores obtenus par le questionnaire ont donné lieu à un premier sous-indice. Pour chaque variable, nous avons calculé la somme des réponses obtenues, selon l'échelle proposée dans le questionnaire, et obtenu une moyenne en divisant cette somme par le nombre d'entreprises répondantes. Cette moyenne a par la suite été intégrée comme sousindice pour la confection du tableau comparatif.

- un deuxième sous-indice réfère au secteur d'activité de l'entreprise (soit 1 : secteur primaire, 2 : secteur secondaire et 3 : secteur tertiaire). Des valeurs différentes attribuées à chaque secteur d'activité paraissent s'imposer, du fait que, de façon générale, l'emploi d'une technologie de pointe est plus fréquent à mesure qu'on s'éloigne du secteur primaire.

- Un troisième sous-indice est une valeur composite qui reflète l'importance de la taille de l'entreprise, référant à : l'âge de l'entreprise, le nombre d'employés et le chiffre d'affaires. Les valeurs associées à ces indicateurs ont été estimées selon les échelles suivantes :

- l'âge de l'entreprise : 3 ans et moins : 1; entre 4 à 6 ans : 2; entre 7 et 9 ans : 3; entre 10 et 12 ans : $4 ; 13$ ans et plus : 5 ; cette valeur est nulle lorsque le répondeur ne sait pas.

- le chiffre d'affaires: 0 à $500000 \$: 1$; de 500001 à $1000000 \$: 2 ;$ de $1000001 \$$ à $1500000 \$$ : 3 ; de 1500001 à 2000001 : 4; de 2000001 à $5000000: 5 ; 5000001$ et plus : 6; quand le répondeur ne sait pas, cette valeur est nulle. 
- le nombre d'employés: entre 0 et 10 employés: 1 ; entre 11 et $25: 2$; entre 26 et $50: 3$; entre 51 et $100: 4 ; 101$ et plus : 5 ; lorsque le répondeur ne sait pas, cette valeur est nulle.

La valeur composite de ces trois variables qui précèdent (âge de l'entreprise, chiffre d'affaires, nombre d'employés) a été divisée par le nombre d'entreprises considérées. Le résultat devient le troisième sous-indice.

La somme des sous-indices constitue lindice qui représente un territoire dans un domaine donné, par exemple les fournisseurs comme sources d'information pour les entreprises.

Dans toutes ces opérations, notre échelle a été fixée entre 0 et 10. En conséquence, quand dans le calcul des sous-indices ou de l'indice final la somme dépasse 10, nous avons divisé par deux tous les résultats se rapportant à la même question, afin de garder les mêmes proportions. Classés sur une échelle de 1 à 10 , ces indices ont servi à préparer les tableaux dynamiques croisés que nous présentons dans les pages qui suivent. L'avantage de cette méthode et de ces tableaux est de permettre de voir, presque en un coup d'œil, en quoi nos territoires se ressemblent et en quoi ils diffèrent, leurs points forts et leurs insuffisances.

Afin de donner une image plus expressive de la situation, nos résultats sont présentés sous forme de graphiques dans la dernière partie de ce chapitre.

\subsubsection{Sources dinformation}

Le tableau 10 indique que, de manière générale, les entreprises implantées dans les quatre territoires confondus, recourent aux différentes sources d'information. La ligne du total moyen des indices calculés montre que, pour les entreprises, les sociétés-conseils $(2,9)$, les visites d'entreprises canadiennes $(2,9)$, les chambres de commerce $(2,8)$, les sociétés de développement $(2,8)$ et les centres de recherche $(2,8)$ sont des sources importantes d'information. 
Tableau 10: Sources d'information

\begin{tabular}{|l|c|l|l|l|l|l|l|l|l|l|l|l|l|l|l|l|}
\hline & 1 & 2 & 3 & 4 & 5 & 6 & 7 & 8 & 9 & 10 & 11 & 12 & 13 & 14 & 15 & 16 \\
\hline Région de Québec & 2,0 & 1,6 & 2,9 & 3,1 & 2,9 & 2,8 & 2,4 & 2,8 & 3,1 & 1,9 & 3,1 & 3,1 & 1,8 & 2,5 & 1,8 & 3,0 \\
\hline Saguenay & 1,9 & 1,4 & 2,6 & 3,0 & 2,5 & 2,5 & 2,4 & 3,0 & 3,1 & 2,3 & 2,8 & 2,7 & 2,0 & 2,4 & 1,7 & 3,0 \\
\hline Manicouagan & 1,9 & 1,4 & 3,3 & 3,5 & 2,8 & 2,4 & 3,0 & 3,2 & 3,7 & 2,2 & 3,1 & 3,1 & 2,0 & 2,2 & 1,5 & 2,5 \\
\hline Rivière-duLLup & 1,6 & 1,1 & 3,0 & 3,2 & 2,6 & 2,6 & 2,4 & 2,9 & 3,1 & 2,5 & 3,2 & 3,0 & 2,5 & 2,5 & 1,9 & 2,9 \\
\hline
\end{tabular}

Source: Complation de l'auteur

$1=$ SFOURN = Les lournisseurs des entreprises;
$2=$ SCLEN = Les clients de l'entreprise;
$3=$ SCENTR = Les centres de recherche;
$4=$ SNIVR = Les universités;
$5=$ SGOUFE = Le gouvernement fédéral;
$6=$ SGOUPR = Le gouvernement provincial;
$7=$ SFOIRE = Les toires et expositions;
$8=$ SVICAN = Visites d'entreprises canadiennes;

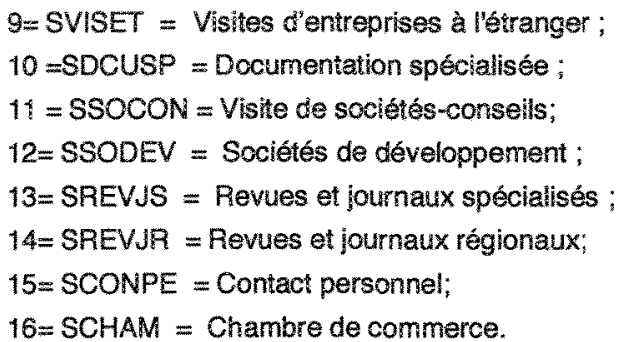

$16=$ SCHAM = Chambre de commerce.

Une analyse attentive de ce tableau ne permet de dégager qu'une différence légère entre les quatre territoires étudiés. Les indices observés pour la région de Québec sont légèrement supérieurs à la moyenne. Toutefois dans certains cas, l'avance des régions périphériques témoigne d'un effort soutenu à s'approprier l'information stratégique : c'est le cas, par exemple, de Manicouagan et de Rivière-du-Loup, avec des indices de 3 en ce qui concerne les sociétés de développement.

\subsubsection{Moyens d'interaction}

Nous avons vu que les entreprises des quatre territoires, tous secteurs industriels confondus, recherchent toutes l'information stratégique. Le tableau 11 permet de voir comment ces entreprises se servent de différents outils pour se procurer et diffuser cette information. Dans ce tableau, on observe des variations selon les moyens et selon les territoires. La moyenne des indices calculés montre que les entreprises des quatre territoires comptent souvent sur le téléphone et le télécopieur pour émettre et pour recevoir l'information stratégique : dans ces cas, l'indice moyen est supérieur à 4; alors qu'il n'est que de 1,5 dans le cas de la place publique. L'indice calculé est autour de 3,7 dans le cas du courriel. Cet indice est de 3,9 au Saguenay, de 3,3 dans la région de Québec et de 3,5 à Rivière-du-Loup. 
Tableau 11: Movens dinteraction

\begin{tabular}{|l|l|l|l|l|l|l|l|l|l|l|}
\hline & 1 & 2 & 3 & 4 & 5 & 6 & 7 & 8 & 9 & 10 \\
\hline Région de Québec & 4,0 & 3,9 & 3,6 & 3,2 & 2,7 & 2,8 & 1,5 & 1,5 & 3,8 & 3,3 \\
\hline Saguenay & 4,8 & 4,9 & 4,6 & 4,5 & 3,2 & 3,2 & 1,3 & 1,3 & 4,0 & 3,9 \\
\hline Manicouagan & 4,2 & 4,3 & 3,9 & 4,0 & 2,9 & 2,8 & 1,8 & 1,8 & 3,8 & 3,6 \\
\hline Rivière-du-Loup & 3,7 & 3,7 & 4,0 & 4,1 & 2,7 & 2,7 & 2,0 & 2,1 & 3,5 & 3,5 \\
\hline
\end{tabular}

Source: Compilation de lauteur

$8=$ TEREC = Téléphone comme moyen de réception d'information;

$2=$ TEEMT = Téléphone comme moyen d'émission d'information;

$3=$ TECREC $=$ Télécopie comme moyen de réception d'information;

$4=$ TELCEMT = Télécopie comme moyen d'émission d'information ;

$5=$ FACEREC = Les activités de face-à-face comme moyen de réception d'information;

6= FACEEMT = Les activités de face-à-face comme moyen d'émission d'information ;

$7=$ PUBLREC $=$ La place publique comme moyen de réception d'information;

$8=$ PUBLEMT = La place publique comme moyen d'émission d'information;

$9=$ COURREC $=$ Le courriel comme moyen de réception d'information ;

$10=$ COUREMT $=$ Le courriel comme moyen d'émission d'information.

Il ressort de ces observations que les entreprises implantées dans les territoires périphériques, comme celles qui sont implantées dans la région de Québec, font un usage judicieux des moyens d'interaction, plus particulièrement des moyens à base d'une technologie de pointe.

On remarque que le téléphone, le télécopieur et le courriel sont des moyens très courants d'interaction, particulièrement au Saguenay et au Manicouagan.

\subsubsection{Types d'information}

Une lecture attentive du tableau 12 montre que certains indices sont inférieurs à 2 ; c'est le cas de l'information sur la concurrence $(1,8)$, sur les nouveaux produits $(1,7)$, sur les technologies $(1,9)$. Ces indices montrent pour les quatre territoires des indices généralement comparables, ce qui veut dire que les entreprises implantées dans les quatre territoires se préoccupent à peu près du même type d'information, qu'elles tentent d'insérer dans leurs processus de gestion. 
Tableau 12: Types dinformation

\begin{tabular}{|l|l|l|l|l|l|l|l|l|l|l|l|}
\hline & 1 & 2 & 3 & 4 & 5 & 6 & 7 & 6 & 9 & 10 & 11 \\
\hline Région de Québec & 2,5 & 1,6 & 1,6 & 2,8 & 2,6 & 1,8 & 2,0 & 2,9 & 3,0 & 2,9 & 2,8 \\
\hline Saguenay & 2,7 & 1,9 & 1,7 & 2,3 & 2,4 & 1,9 & 2,0 & 2,5 & 3,4 & 2,5 & 1,9 \\
\hline Manicouagan & 2,7 & 2,4 & 2,1 & 3,4 & 3,1 & 2,2 & 1,9 & 2,8 & 1,9 & 2,7 & 2,5 \\
\hline Rivière du Loup & 1,9 & 1,5 & 1,4 & 2,5 & 2,6 & 1,9 & 1,7 & 3,3 & 2,7 & 2,5 & 2,6 \\
\hline
\end{tabular}

Source: Compllation de lauteur

$1=$ INFOCONJ = La conjoncture:

$2=1 N F C O N C=$ La concurrence ;

$3=$ INFNVPRO $=$ Les nouveaux produits;

$4=$ INFEXPOR $=$ Les exportations ;

$5=$ INFSCIEN $=$ L'information scientifique ;

$6=$ INFTECHO=L'information technologique
$7=$ NFTECHI = information technique;

$8=$ INFNOJU $=$ L'information d'ordre normatt, juridique.

$9=$ INFSECUR $=$ La sècurité;

$10=$ INFRESPU $=$ Les ressources publiques;

$11=$ INFOMEDGE = Les modalités de gestion.

Cependant, l'ensemble de ces indices montre que les entreprises implantées dans les territoires de Québec et de Saguenay accordent une importance plus grande aux questions reliées à la sécurité : l'indice calculé dans le cas de Québec est de 3, il est 3,3 au Saguenay, contre seulement 1,9 à Manicouagan et 2,6 à Rivière-du-Loup. Quant à la recherche d'information relative à l'exportation, les entreprises de Manicouagan se démarquent par un indice bien supérieur à la moyenne : 3,4, comparativement à 2,8 à Québec et 2,3 et 2,5 dans les deux autres territoires. Plus qu'ailleurs, on recherche l'information de nature normative et juridique à Rivière-du-Loup.

Ces données font voir que les entreprises implantées en territoire périphérique cherchent à se doter des stratégies susceptibles de les insérer dans le nouvel ordre économique.

\subsubsection{Activités extérieures}

Les résultats concernant l'implication des dirigeants d'entreprises dans les activités extérieures sont présentés dans le tableau 13. Les moyens extérieurs d'interaction les plus importants que les entreprises des quatre territoires utilisent sont les causes caritatives et 
l'environnement (3) et les clubs sociaux (3). C'est au Saguenay que nous avons identifié l'indice le plus important quant à ces clubs (3) comparativement à 2,9 à Québec et Rivière-duLoup et à 2,7 à Manicouagan. Pour ce qui est des causes caritatives, cet indice dépasse 3 dans les quatre territoires.

Tableau 13: Activités extérieures

\begin{tabular}{|l|c|c|c|c|c|c|c|c|c|}
\hline & 1 & 2 & 3 & 4 & 5 & 6 & 7 & 8 & 9 \\
\hline Région de Québec & 3,2 & 3,0 & 2,9 & 2,4 & 3,0 & 3,3 & 2,8 & 3,0 & 2,2 \\
\hline Saguenay & 3,1 & 3,0 & 2,8 & 2,6 & 3,0 & 3,2 & 2,8 & 3,1 & 2,1 \\
\hline Manicouagan & 3,1 & 2,8 & 2,8 & 2,8 & 3,0 & 3,1 & 2,7 & 2,8 & 2,3 \\
\hline Rivière du Loup & 3,3 & 3,1 & 2,8 & 2,5 & 2,8 & 2,7 & 2,6 & 2,8 & 2,4 \\
\hline
\end{tabular}

Source: Compliation de lauteur

$1=$ ACTCARI = Causes caritatives:

6=ACTENVIR $=$ Les activités liées à l'environnement ;

$2=$ ACTEVNMM = Évènements mondains ;

$7=A C T E S P O R=$ Les activités sportives;

$3=$ ACTEVNMS $=$ Les évènements spéciaux ;

$4=A C T E X P O=L$ Le expositions ;

$8=$ ACTECLUS $=$ Les activités organisées dans clubs sociaux;

$5=A C T E R E C O=L$ Les activités de

$9=A C T E R E P A=$ Les repas.

reconnaissance.

Par ailleurs, l'indice moyen le plus faible est observé dans le cas des repas $(2,2)$ et des expositions $(2,6)$. Pour les repas, on trouve lindice le plus faible au Saguenay et le plus élevé à Rivière-du-Loup, mais la différence est peu importante. Par contre les évènements mondains et de reconnaissance comptent passablement comme activités extérieures.

Dans les quatre territoires, les dirigeants d'entreprises semblent en général choisir les activités extérieures qui comptent le plus en termes de contribution sociale et aussi, souvent sans doute, de visibilité. Quel que soit le territoire d'implantation d'une entreprise, le choix des moyens $d^{\prime}$ interaction semble se faire selon certains paramètres qui ne sont pas propres aux territoires, mais dépendent plutôt de la formation antérieure des entrepreneurs et de la culture organisationnelle existante. 


\subsubsection{Moyens de communication interne}

Nous avons voulu comparer les quatre territoires sur la base des moyens de communication interne qu'adoptent les dirigeants d'entreprises. Les résultats obtenus sont présentés dans le tableau 14. Les indices moyens obtenus varient entre 2 et 7 , montrant que les réunions du personnel demeurent le moyen le plus utilisé pour faire véhiculer l'information stratégique au sein des organisations; cette observation est valable pour les quatre territoires étudiés. En ce qui concerne le bulletin interne, les indices les plus élevés ont été obtenus dans le cas de Québec et du Saguenay: plus de 3 dans les deux cas. Quant au tableau de nouvelles, c'est au Saguenay que l'on rapporte l'indice le plus important: 5 ; contre 3 à Québec et 2 à Manicouagan et 1 à Rivière-du-Loup.

Le bulletin interne et le tableau de nouvelles sont moins utilisés qu'ailleurs à Rivière-du-Loup, mais l'horaire de mise en commun l'est davantage. Quand à l'Intranet c'est de loin dans la région de Québec qu'il est le plus utilisé.

Tableau 14: Moyens de communication interne

\begin{tabular}{|l|l|l|l|l|l|}
\hline & 1 & 2 & 3 & 4 & 5 \\
\hline Région de Québec & 3,1 & 3,1 & 4,1 & 7,9 & 1 \\
\hline Saguenay & 3,5 & 5 & 2 & 7,3 & 1,2 \\
\hline Manicouagan & 2,8 & 2,5 & 0,8 & 8,3 & 0,8 \\
\hline Rivière du Loup & 1,5 & 1,5 & 0,8 & 6,2 & 2,3 \\
\hline
\end{tabular}

Source: Compilation de lauteur

$1=S Y S C O M B U L=$ Bulletin interne;

$2=$ SYSCOMTN $=$ Tableau de nouvelles ;

$3=S Y S C O M I N=$ Intranet;

$4=S Y S C O M R P=$ Réunion du personnel ;

$5=S Y S C O M H C=$ Horaire de mise en commun.

En général, considérant l'ensemble des moyens de communication interne utilisés par les entreprises, on constate qu'il n'y a pas d'écart significatif entre les quatre territoires étudiés, sauf pour IIntranet. Si llntranet est plus fréquent dans la région de Québec, c'est que les entreprises y sont souvent plus importantes ou qu'elles ont plus d'une place d'affaires. 


\subsubsection{Classement de linformation}

Deux préférences ressortent du tableau 15. D'abord que certains moyens de classement de l'information sont très répandus, avec des indices significatifs; le classement par sujet et par projet : 5 dans les deux cas. Le classement informatique suit avec un indice de 3 ; alors que les autres méthodes de classement ont des indices faibles: c'est le cas, par exemple, du classement par date $(0,8)$, du classement dans une bibliothèque de l'organisation $(0,7)$. Ensuite, on observe un écart significatif entre les indices enregistrés dans les différents territoires. Dans le cas du classement de l'information par sujet, l'indice le plus élevé est observé à Manicouagan (6) comparativement à 5 à Québec, et 4 au Saguenay et 3 à Rivièredu-Loup.

Tableau 15: Classement de Information

Source: Compilation de lauteur

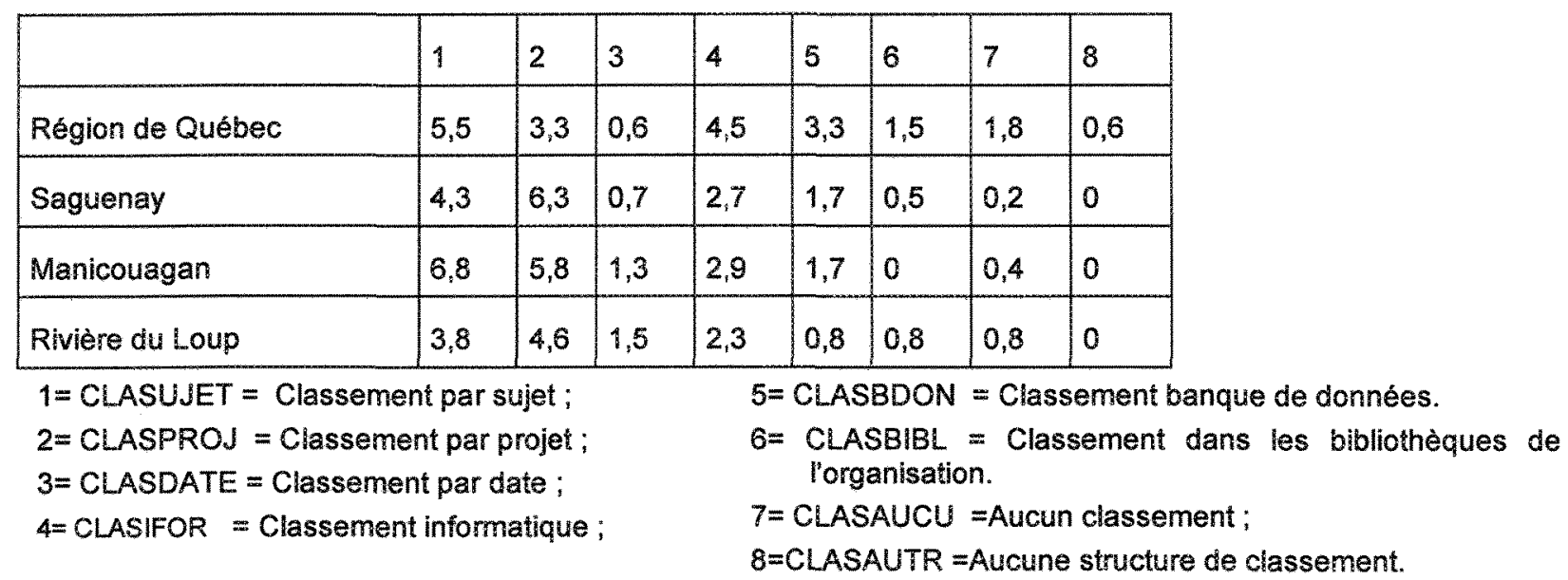

Les systèmes de classement de l'information utilisés dans les territoires périphériques se ressemblent passablement ; mais sauf pour le classement par sujet et par projet, ils diffèrent sensiblement des moyens utilisés à Québec où, par exemple, on emploie davantage le classement informatique ou dans une banque de données. 


\subsubsection{Soutien extérieur}

Le tableau 16 montre que l'on accorde une importance particulière aux conseillers et fournisseurs en matériel et en logiciel, avec un indice de 7,8 à Manicouagan; c'est l'indice le plus important, alors qu'au Saguenay il est de 6, suivi de Québec (5) puis de Rivière-du-Loup $(4,6)$. Dans le cas des foumisseurs d'accès à Internet, l'indice le plus important est observé à Québec (5), suivi de Rivière-du-Loup et de Manicouagan (4) et du Saguenay (2). C'est à Québec que l'on trouve l'indice le plus élevé en ce qui concerne l'hébergeur de sites Web (4) et de créateur de page Web (4); alors que c'est à Rivière-du-Loup que l'on observe l'indice le plus élevé en ce qui conceme les conseillers en communication (3) et à Manicouagan pour les formateurs.

Tableau 16: Soutien extérieur

\begin{tabular}{|l|l|l|l|l|l|l|l|}
\hline & 1 & 2 & 3 & 4 & 5 & 6 & 7 \\
\hline Région de Québec & 1,5 & 5,4 & 5,4 & 2,7 & 1,9 & 4,2 & 3,8 \\
\hline Saguenay & 1,4 & 6,1 & 2,2 & 2,4 & 1 & 1,4 & 2,5 \\
\hline Manicouagan & 1,3 & 7,8 & 3,5 & 1,7 & 3 & 0,4 & 0,9 \\
\hline Rivière-du- Loup & 0,8 & 4,6 & 3,8 & 3,1 & 2,3 & 2,3 & 3,1 \\
\hline
\end{tabular}

Source: Complation de lauteur

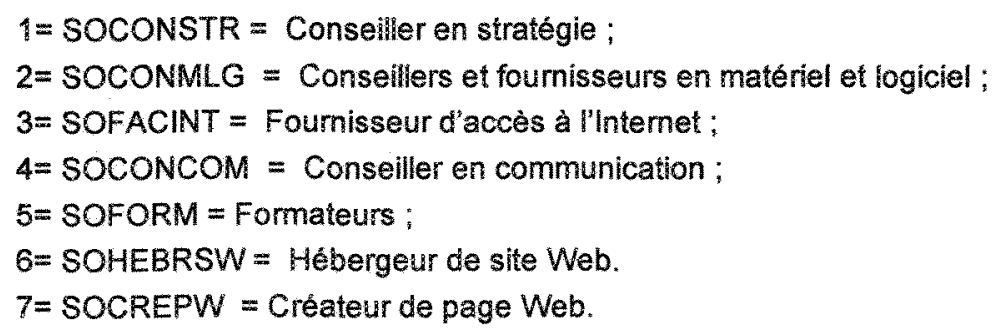

De façon générale, les conseillers en stratégie sont assez peu en demande dans l'ensemble des territoires; dans Manicouagan les conseillers en communication, les hébergeurs de site Web et les créateurs de pages Web sont peu utilisés. Au Saguenay, ce sont les formateurs qui sont peu consultés. 


\subsubsection{L'usage de l'mternet}

$\mathrm{Au}$ tableau 17, l'indice le plus élevé revient à l'envoi de messages (8); viennent ensuite l'acquisition de données stratégiques (6), liaison avec les fournisseurs (5) l'offre de produits et services et repérage de nouveaux contacts, (4). En ce qui concerne l'envoi de messages, l'indice le plus élevé revient à la région de Québec et au Manicouagan (9), puis au Saguenay (8). Dans le cas de l'acquisition de données, l'indice le plus élevé, soit 7, est observé dans la région de Québec et de Saguenay; alors que l'indice le plus élevé dans le cas des liaisons avec les fournisseurs s'applique à la région de Québec (6), suivie de Saguenay (5), de Manicouagan (4) et de Rivière-du-Loup (3).

Tableau 17: Usage de Irnternet

\begin{tabular}{|l|l|l|l|l|l|l|l|l|l|l|l|l|l|}
\hline & 1 & 2 & 3 & 4 & 5 & 6 & 7 & 8 & 9 & 10 & 11 & 12 & 13 \\
\hline Région de Québec & 9,4 & 7,6 & 4,8 & 4,8 & 6,7 & 4,2 & 4,8 & 5,8 & 2,1 & 4,5 & 3,3 & 5 & 4,1 \\
\hline Saguenay & 8,8 & 7,6 & 3,1 & 3,6 & 5,3 & 2,6 & 2,1 & 4,3 & 1,6 & 5,2 & 1 & 3,5 & 1,4 \\
\hline Manicouagan & 9,2 & 5,4 & 2,1 & 0,8 & 4,6 & 1,3 & 0,8 & 4,2 & 0 & 2,1 & 2,5 & 1,2 & 1,6 \\
\hline Rivière-du-Loup & 7,7 & 4,6 & 2,3 & 0 & 3,1 & 0,8 & 1,5 & 2,3 & 0,8 & 2,3 & 0,8 & 1,5 & 0 \\
\hline
\end{tabular}

Source: Compilation de lauteur

$1=$ UMAIL = Envoi de messages ;

$2=U C Q D O N=$ Acquisition de données ;

$3=$ URICOM $=$ Prise de commandes;

$4=$ UOMINT $=$ Communication interne-gestion ;

$5=$ UIAIF = Liaison avec les foumisseurs;

$6=$ UIVRRE $=$ Livraison et réception de produits ;

$7=U E R S O N=$ Personnel $=$ ouverture sur le monde.

\author{
$8=$ UEPRAG $=$ Repérage de nouveaux contacts \\ $9=$ UCURDO = Sécurisation de données; \\ $10=$ UEVTR $=$ Site vitrine ; \\ $11=$ UEGFIN = Règlement financier; \\ $12=$ UCHA : achats sur le web; \\ $13=$ UPOWE: usage du potentiel du Web
}

En ce qui concerne l'usage du potentiel du Web, le total des indices observés est égal à 2 . C'est à Québec que l'on a observé l'indice le plus élevé (4), contre l'indice 0, observé à Rivière-du-Loup. En fait, si les possibilités du Web pourraient être exploitées bien davantage, il s'y fait néanmoins des achats dans tous les territoires en quantité appréciable, particlièrement à Québec. Pour ce qui est de la gestion, l'Internet est utilisé modérément à Québec et au Saguenay, peu à Manicouagan et en aucun cas à Rivière-du-Loup. 
générale on ne lésine pas sur le matériel informatique. Dans tous les territoires se trouvent diverses sortes d'ordinateurs et une variété de périphériques adaptés à des tâches spécifiques. Entre les quatre territoires, on ne voit pas de disparités très accentuées.

\subsubsection{L'appropriation des logiciels}

Le tableau 19 montre que l'indice le plus élevé a été observé dans le cas des logiciels que l'on utilise pour le traitement de texte (9): presque toutes les entreprises implantées à Manicouagan et à Rivière-du-Loup qui ont complété notre questionnaire utilisent un logiciel de traitement de texte; l'indice étant de 10. La même constatation vaut quant à l'usage des logiciels de comptabilité. En ce qui concerne le chiffrier électronique, l'indice moyen obtenu est de 9 : nous avons observé l'indice le plus élevé à Rivière-du-Loup (10), suivi de Québec et de Saguenay (9) et de Manicouagan (8). Les logiciels utilisés pour la gestion de projet sont plus utilisés à Québec et à Manicouagan (7) qu'au Saguenay (5) et à Rivière-du-Loup (4).

Tableau 19: Appropriation des logiciels

\begin{tabular}{|l|l|l|l|l|l|l|l|l|l|l|}
\hline & 1 & 2 & 3 & 4 & 5 & 6 & 7 & 8 & 9 & 10 \\
\hline Région de Québec & 7,5 & 9,7 & 9,7 & 6,8 & 8,5 & 9,1 & 7,3 & 5,4 & 7,7 & 5 \\
\hline Saguenay & 3,5 & 9,3 & 9,5 & 5,9 & 8,9 & 9,5 & 5,8 & 2,5 & 6,1 & 0 \\
\hline Manicouagan & 5,6 & 10 & 10 & 7 & 7,4 & 8,6 & 7,4 & 4,3 & 4,5 & 10 \\
\hline Rivière-du-Loup & 5 & 10 & 10 & 4,5 & 9 & 10 & 4 & 4 & 8,2 & 0 \\
\hline
\end{tabular}

Source: Compilation de lauteur

$1=$ LOGEDI = Les logiciels d'échange d'information;

$2=$ LOGTRAIT = Les logicieis de traitement de texte

$3=$ LOGCOMP $=$ Les logiciels de comptabilite ;

$4=L O G E S T P R=L e s$ logiciels de gestion du personnel;

$5=$ LOGBASED $=$ Les logiciels de gestion de bases de données.

$6=$ LOGCHFEL = Les chiffriers électroniques;

$7=$ LOGETPRO = Les logiciels de gestion de projet;

= LOGSYSEX= Les systèmes experts;

$9=$ LOGESINV = Les logicieis de gestion de linventaire;

$10=$ LOGAUTRE $=$ Autres . 
Ce tableau ne montre pas de disparités importantes entre les territoires quant à l'appropriation d'une variété de logiciels.

Les tableaux dynamiques croisés qui précèdent établissent, pour chacun des thèmes considérés, des comparaisons chiffrées entre les territoires. Afin de parfaire l'analyse, il a paru utile d'examiner ces mêmes données à partir d'un autre point de vue, en les présentant sous forme de graphique. A cette fin un indice global a été calculé pour chacun des territoires, par rapport à chacun des thèmes.

Le graphique 15 montre que les entreprises implantées dans les quatre territoires utilisent presque au même degré les diverses sources d'information; les résultats obtenus varient entre 6,6 , observé dans le cas du Saguenay, et 7 obtenu à Manicouagan. On constate que l'indice obtenu à Rivière-du-Loup et dans la région de Québec est de 6,8 dans les deux cas. Pour ce qui est des sources d'information, les territoires périphériques ne semblent pas être en retard par rapport à la métropole considérée (Québec).

Figure 15: Comparaison des quatre territoires de l'étude à partir d'un indice global : Sources d'information

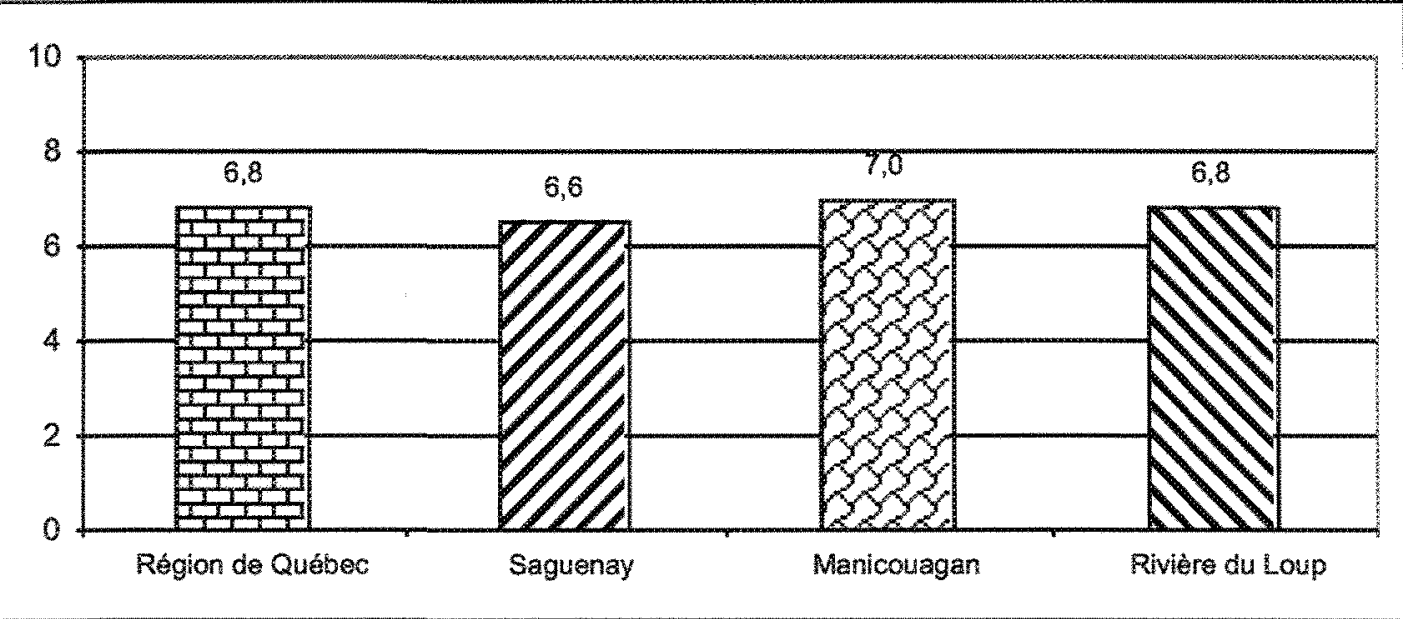


Concernant les moyens d'interaction que sont, pour les dirigeants d'entreprises, le téléphone, le télécopieur, le courriel et autres, le graphique 16 montre que le Saguenay se démarque légèrement, tandis que Québec a l'indice le plus faible, les deux autres territoires étant presque à égalité.

Figure 16: Comparaison des quatre territoires: Moyens d'interaction

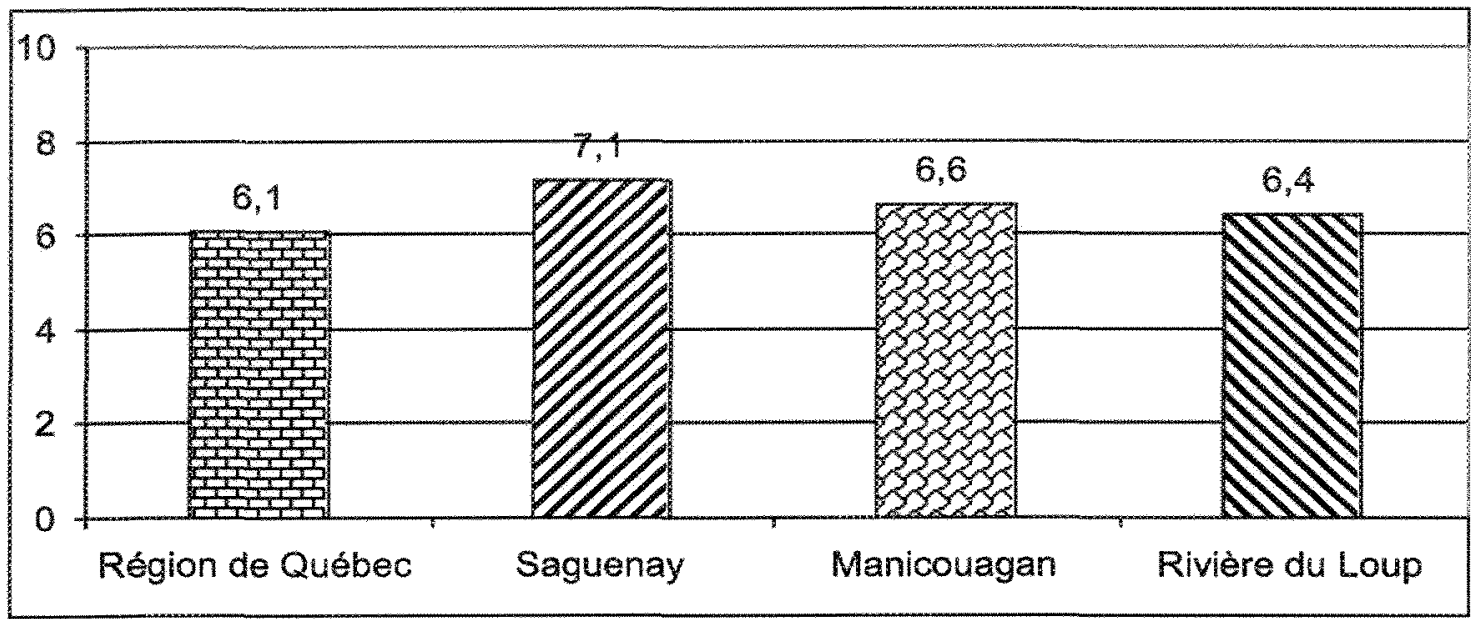

L'information recherchée se rapporte en une dizaine de catégories: concurrence, exportation, sécurité, etc. Le graphique 17 fait ressortir plusieurs sous-indices plus faibles pour Rivière-duLoup et Saguenay. L'indice le plus élevé, pour Manicouagan, témoigne de l'effort des dirigeants locaux pour bien s'informer.

Figure 17: Comparaison des quatre territoires: Types d'information

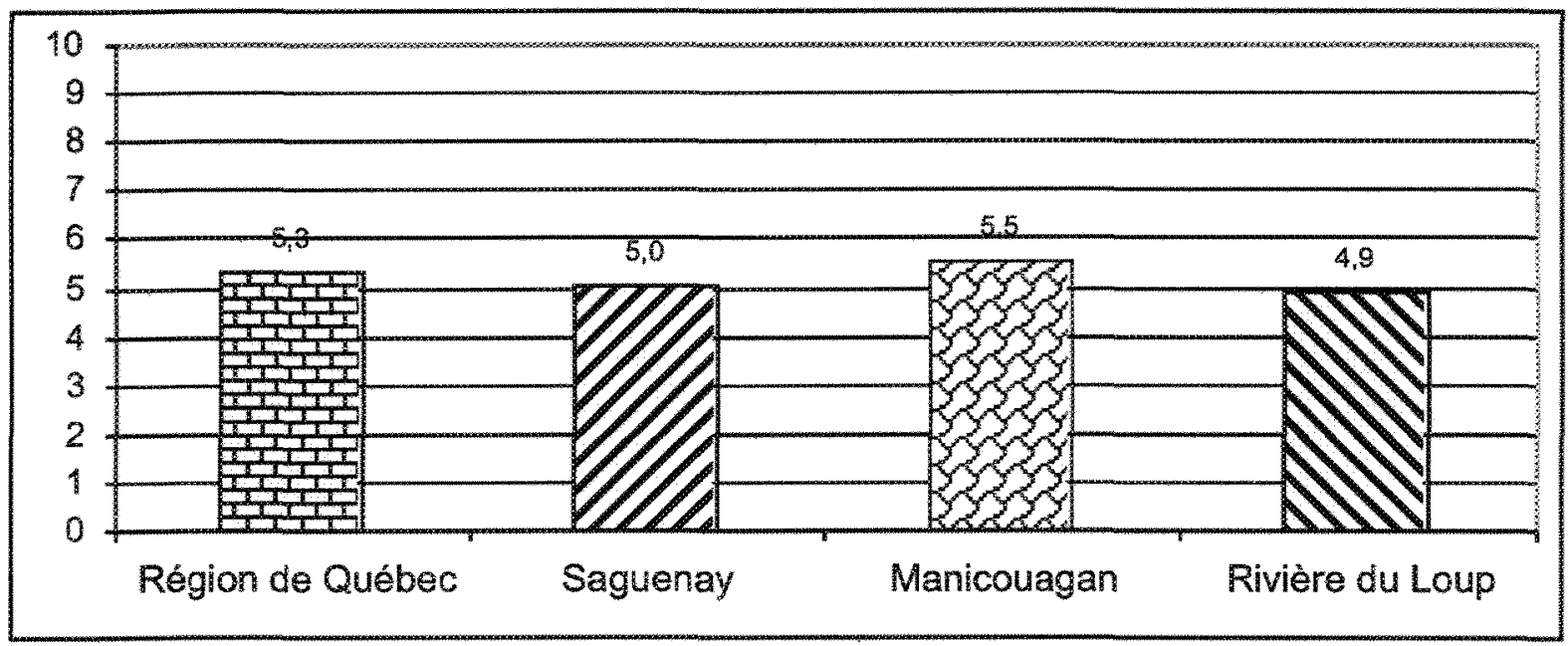


Figure 18: Comparaison des quatre territoires: Activités extérieures

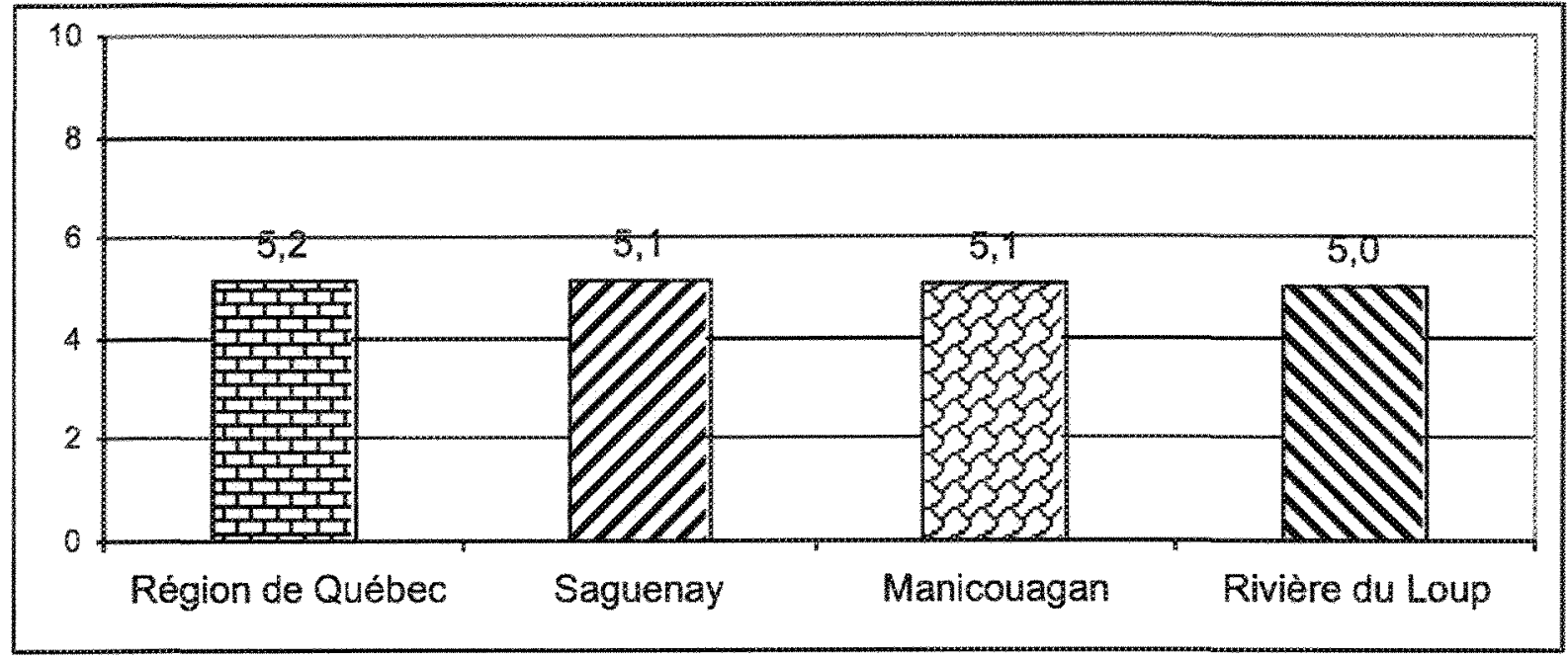

Les activités extérieures amènent les chefs d'entreprises à sortir de leurs bureaux pour se mêler à diverses activités, qui peuvent être favorables au développement, soit de leur milieu, soit de leurs affaires. Le graphique 18 ne montre pas de différences significatives entre les territoires; les dirigeants d'entreprises, indépendamment de leur lieu d'opération, ont sensiblement le même comportement.

Figure 19: Comparaison des quatre territoires: Moyens de communication interne

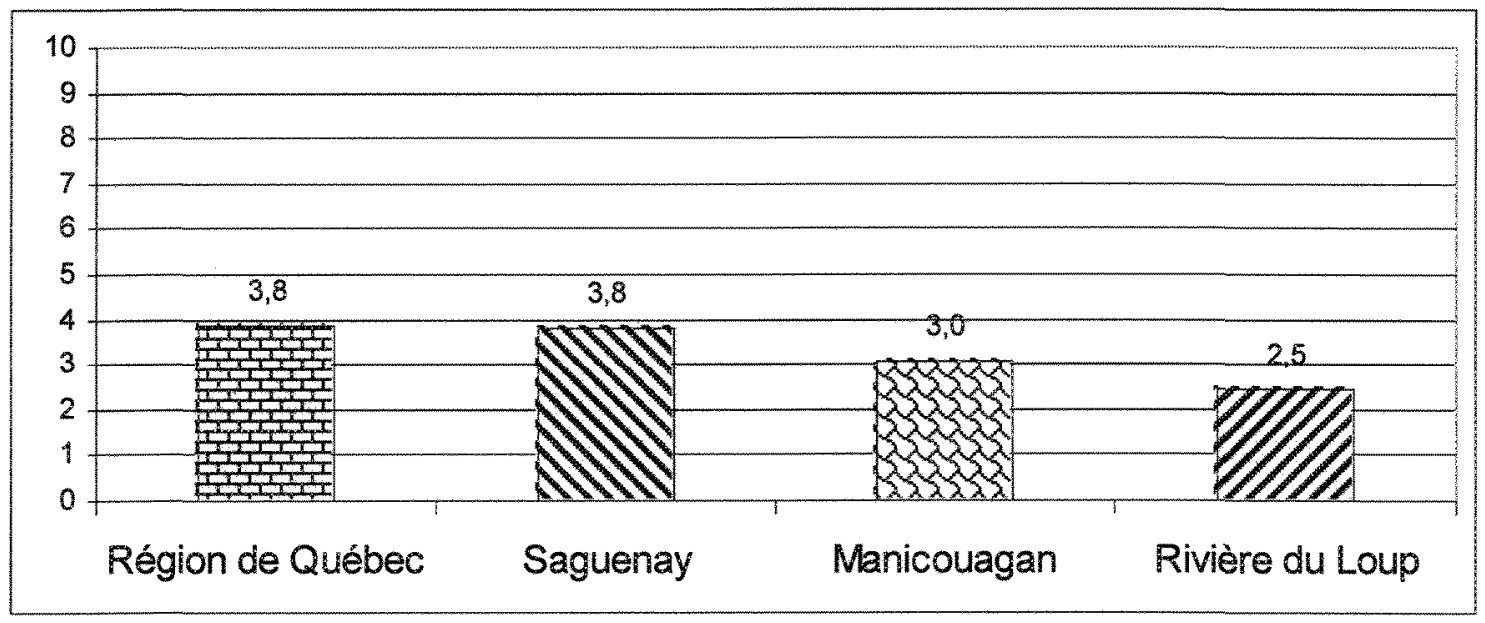

Selon ses besoins et selon sa taille, une entreprise peut avoir plus d'un système de communication interne. A cet égard, et comme le montre le graphique 19, il y a parité entre 
Québec et Saguenay. Manicouagan et surtout Rivière-du-Loup ont des systèmes de communication interne moins développés.

Figure 20: Comparaison des quatre territoires: Classement de l'information

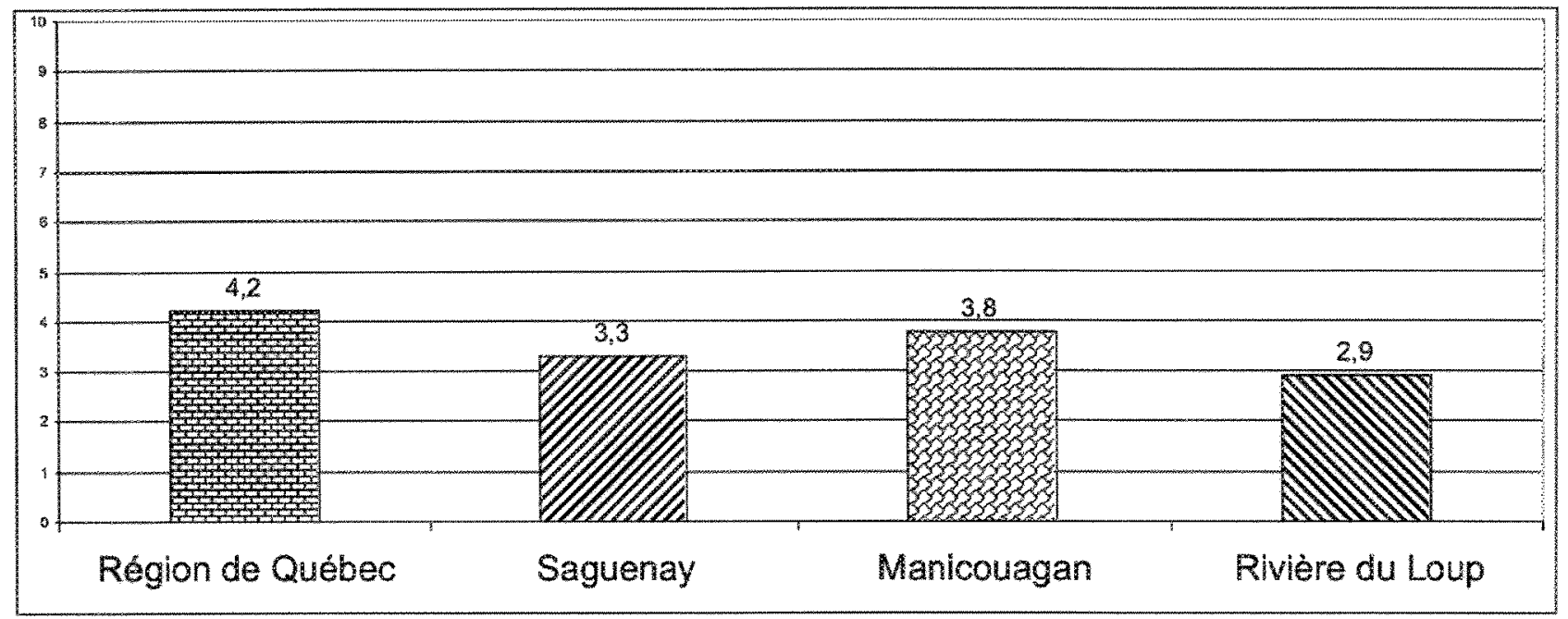

Le graphique 20 illustre les résultats concernant le classement de l'information. Des indices plus élevés pour Québec et Manicouagan reflètent plus de variété dans les systèmes de classement de l'information. Pour Saguenay et Rivière-du-Loup, un classement apparemment moins sophistiqué correspond à des besoins différents.

Figure 21: Comparaison des quatre territoires: Soutien extérieur

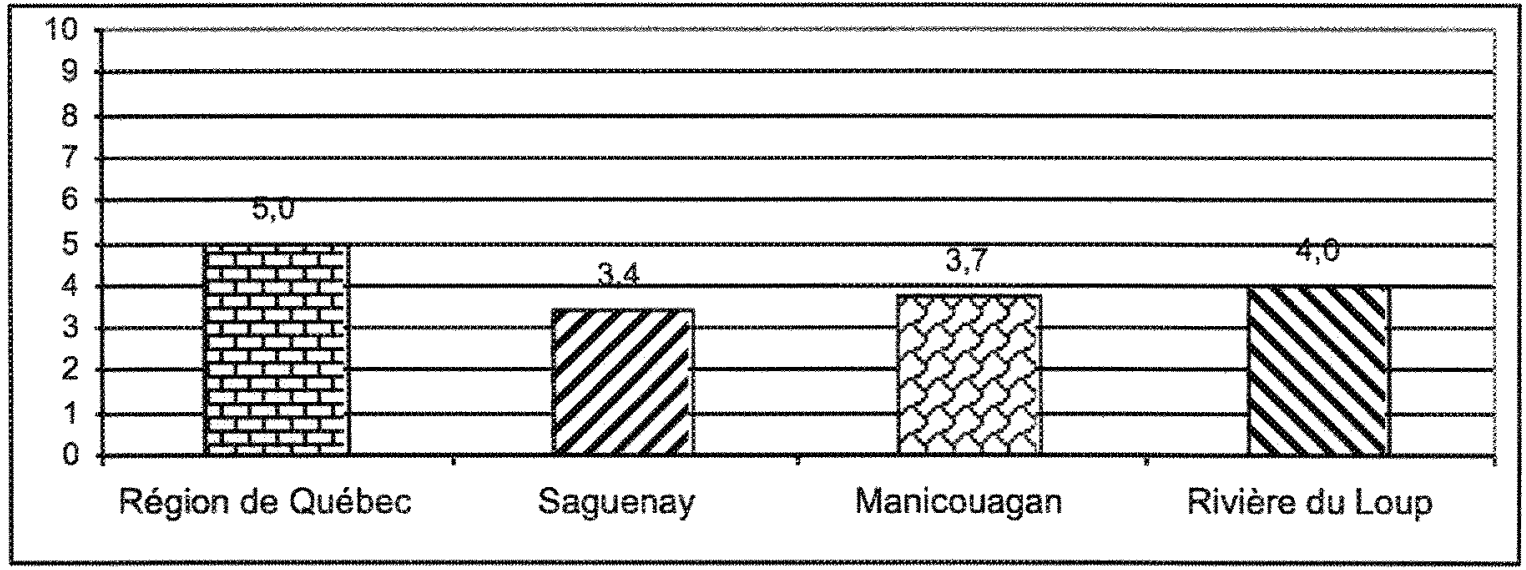


Le graphique 21 représente l'intensité avec laquelle les entreprises font appel à des ressources extérieures pour la résolution de problèmes et la planification stratégique. Manicouagan et davantage Saguenay tirent un peu de l'arrière.

Figure 22: Comparaison des quatre territolres: L'usage de l'Internet

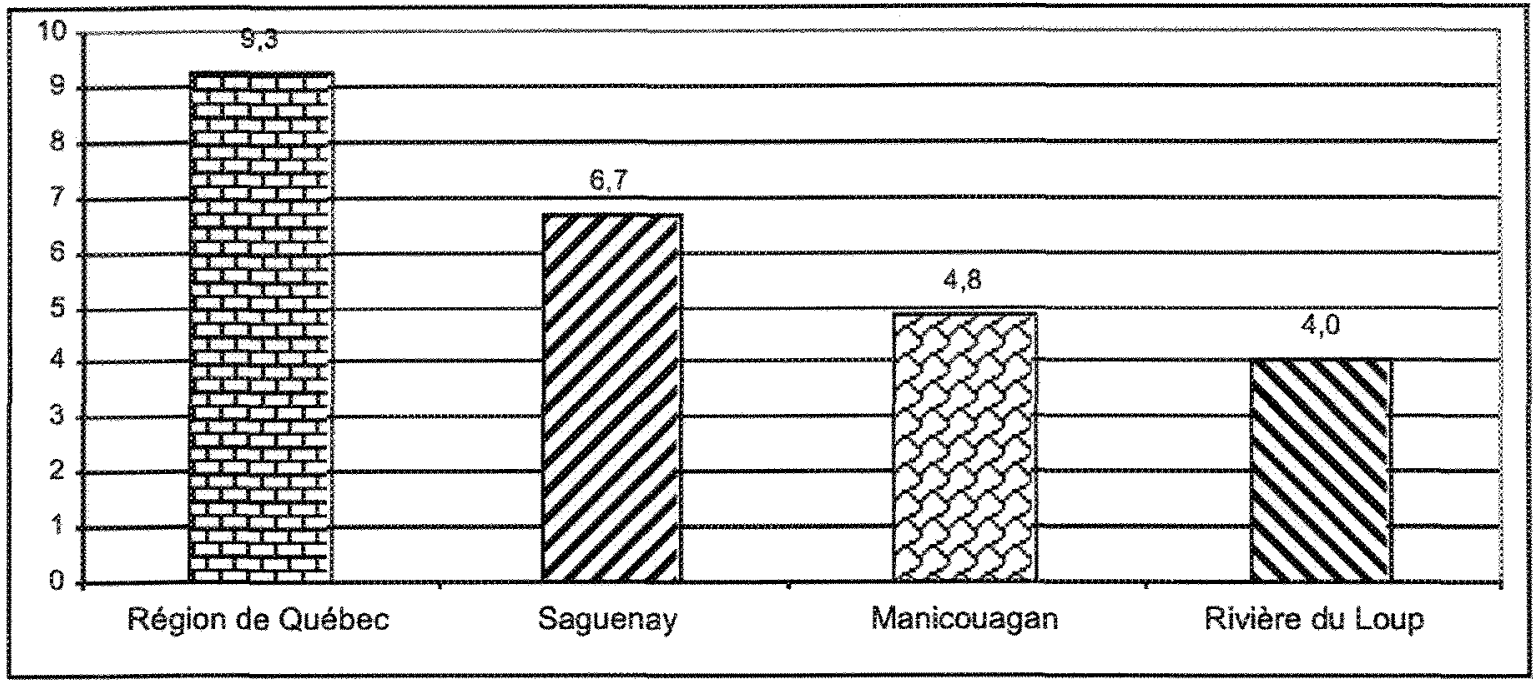

Le graphique 22 montre que la région de Québec se démarque considérablement quant à l'intensité et à la variété des usages d'Internet. Saguenay fait assez bien mais les deux autres territoires ont droit à du rattrapage.

Figure 23: Comparaison des quatre territoires: Appropriation du matériel informatique et des logiciels

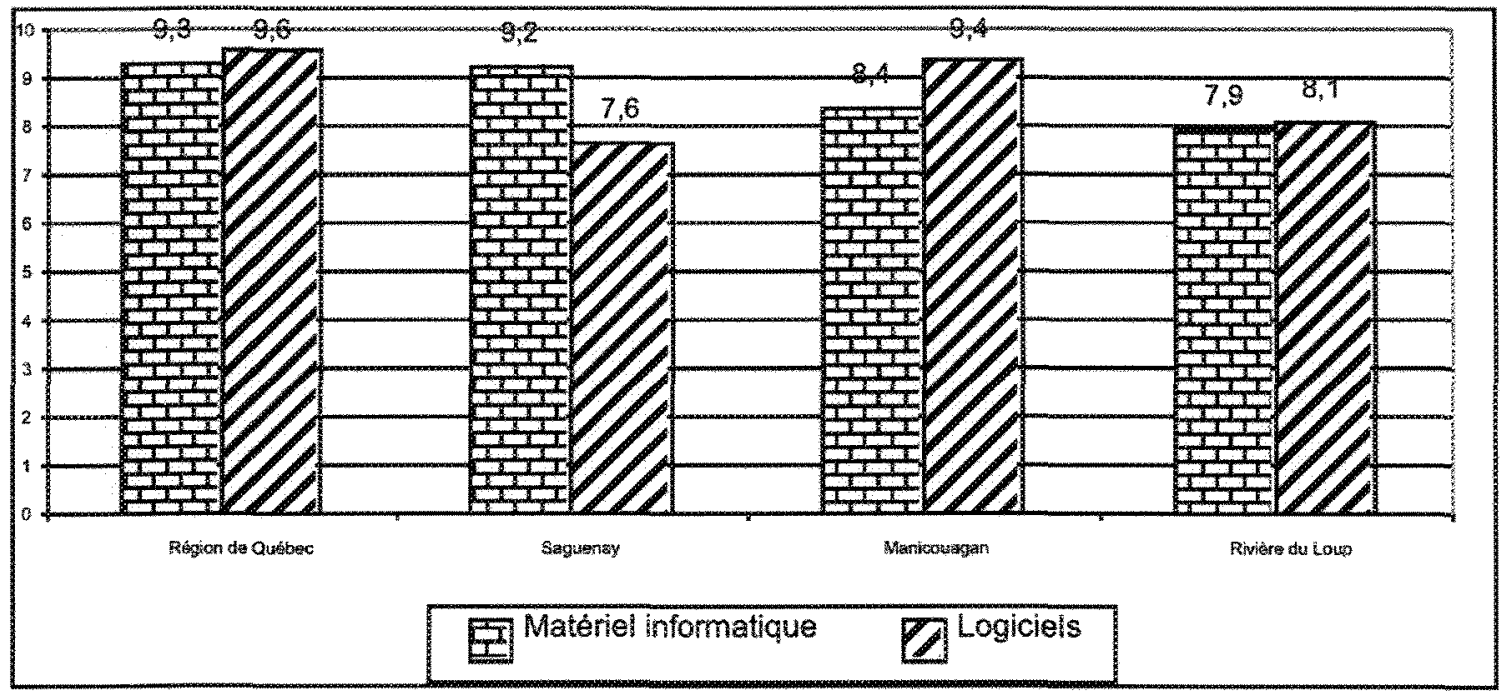


Le graphique 23 permet de comparer les quatre territoires en ce qui concerne l'appropriation du matériel informatique et des logiciels. Les indices élevés de ce graphique indiquent que l'appropriation de l'informatique est raisonnablement bonne partout, bien que des progrès soient souhaitables, particulièrement à Rivière-du-Loup. Manicouagan retarde un peu du côté des matériels et Saguenay quant aux logiciels.

\section{Conclusion}

Il est remarquable que, dans l'ensemble, on recherche peu linformation scientifique, l'information sur la sécurité des procédés et des données, et qu'on consulte assez peu les centres de recherche et les universités. Par ailleurs on pourrait aller plus loin dans l'appropriation des nouvelles technologies et surtout exploiter davantage les possibilités du Web.

$\mathrm{Au}$ départ de cette recherche, les territoires sous observation différaient sous plusieurs aspects, notamment par le caractère central ou périphérique, le nombre et la taille des entreprises, le secteur d'activité, etc. L'examen de nos données a montré néanmoins que le facteur régional joue très peu quant au fonctionnement des entreprises. Ce que nous appelons facteur régional réfère à l'importance de l'effet d'une même variable dans des régions différenciées.

La manipulation de nos données a fait ressortir par exemple que l'effet de la taille d'une entreprise est le même dans tous les quatre territoires; que les effets des variables explicatives sur le choix de diverses sources d'information est le même pour tous les territoires étudiés.

En somme, l'appartenance à un territoire ou l'autre a très peu d'influence sur les attitudes et les comportements, sur l'usage que font les acteurs de développement des objets technologiques et de l'information. Les écarts entre les territoires sont cependant évidents, mais ne $s^{\prime}$ expliquent guère par les actions et les pratiques des gens d'affaires qui y travaillent. Il appert que les sources du retard des territoires périphériques sont à chercher à d'autres niveaux et que l'appropriation maximale des TIC peut avoir une influence décisive pour corriger la situation. 
Ce chapitre fait voir que l'arrivée massive des TIC et la généralisation d'une économie de linformation font que les dirigeants adoptent presque les mêmes pratiques et les mêmes comportements et que le même savoir-faire se généralise partout. Par conséquent, tout donne à penser que le facteur régional ne peut justifier à lui seul le retard des territoires périphériques par rapport aux métropolitains. Cette réflexion sera poussée davantage dans le chapitre qui suit. 


\section{CHAPITRE 8.}

\section{LEÇONS CONCURRENTES TIRÉES DE L'ANALYSE ET DES OBSERVATIONS SUR LE TERRAIN}

L'analyse faite au chapitre précédent a mis en relief certains aspects importants du fonctionnement des entreprises, qu'il convient d'interpréter à la lumière des pratiques observées sur le terrain. C'est le propos du présent chapitre, qui comprend deux sections. La première est consacrée à quelques caractéristiques du vécu entrepreneurial. La deuxième section porte sur les enseignements qu'il nous parât possible de tirer de cette étude.

\subsection{Le vécu entrepreneurial}

Cette section est réservée aux leçons que nous tirons des pratiques d'affaires observées dans les quatre territoires retenus pour les fins de cette thèse. Ces leçons concernent notamment l'appropriation du matériel informatique et des logiciels, les effets de la taille des entreprises, l'importance des affaires électroniques.

\subsubsection{Le matériel informatique et les logiciels}

La prise de décision par le dirigeant pour la conduite de son entreprise est un processus continu qui requiert une information pertinente et à jour dans la gestion quotidienne comme 
pour la planification stratégique. Il a constamment besoin de données sur le marché, la concurrence, les fournisseurs, les clients, les programmes gouvernementaux, les techniques et technologies nouvelles qui apparaissent dans son champ d'activité. La possession de ces renseignements est toujours utile, parfois vitale pour l'entreprise. Elle fournit des éléments de comparaison, met en lumière des changements à considérer et fait voir des solutions possibles.

Accompagnant l'acquisition de données, l'identification des besoins et le choix des solutions appropriées, interviennent l'expérience et le savoir-faire du dirigeant, et l'ensemble des compétences offertes par ses adjoints et par ses réseaux de soutien. La diversité de ces appuis l'aide à mieux cerner ses besoins, à les hiérarchiser selon ses priorités et à prendre des décisions opportunes.

L'expérience, c'est l'accumulation des réussites, et aussi (parfois) des échecs. Aujourd'hui toutefois la seule expérience n'est pas garante de réussite. L'évolution difficilement prévisible de la conjoncture impose des réactions rapides et appropriées. L'utilisateur du Web a plus de possibilité de "voir venir" et de se préparer. Et si, dans l'entreprise, on est déjà familiarisé avec un certain équipement informatique, on cherchera à en augmenter l'efficacité en même temps que la compétence des utilisateurs. Le dirigeant constatera assez souvent l'insuffisance de ses systèmes d'information, de gestion ou de communication, et ce seront alors les composantes de ces systèmes indispensables à l'efficience des activités quotidiennes, qui seront d'abord renforcées.

Le dirigeant d'entreprise voit ordinairement l'intérêt d'évaluer régulièrement la pertinence et l'efficacité de ses systèmes de communication, interne et externe, et de gestion. Cette évaluation peut mener à la recherche de renseignements en vue d'introduire de nouveaux modes de fonctionnement, qu'un matériel informatique plus puissant, des logiciels diversifiés et plus performants rendent possibles. Les processus liés à la gestion quotidienne des opérations tendent alors à se systématiser et la productivité à s'accroître.

En définitive, l'intuition de l'entrepreneur a beaucoup à voir avec ses décisions et la conduite de son organisation. Mais pour se prémunir contre les aléas inhérents au monde des affaires, plutôt que de les subir, l'intuition doit s'appuyer sur des données fiables, actuelles, fournies 
par un système informationnel et communicationnel efficace. $\grave{A}$ cet égard notre analyse montre que l'utilisation de la Toile encourage l'appropriation des outils informatiques de pointe, qu'inversement cette appropriation favorise l'usage du Web.

Nous avons constaté que l'introduction d'un Intranet dans une entreprise invite à la mise en œuvre de technologies avancées. Implanter un Intranet, c'est aussi profiter d'un ensemble de réseaux de communication, de nouveaux procédés qui permettent de recevoir l'information, de la stocker et de la diffuser en temps opportun au sein de l'organisation.

Il y a aussi la culture numérique, qui grandît avec l'implantation d'un Intranet et se reflète sur l'attitude et le comportement du personnel. L'usage d'un Intranet témoigne de l'existence d'une culture managériale tournée vers l'avenir; ce système de communication correspond en effet à un ensemble de processus de gestion axés sur l'emploi d'outils informatiques.

$\mathrm{Si}$, pour toute entreprise relativement importante, l'Intranet s'avère très utile, il est devenu pratiquement indispensable pour celle qui a plus d'une place d'affaires. L'une des fonctions d'un Intranet est de faciliter les communications au sein de l'entreprise : pour la circulation de l'information, la discussion et l'échange des idées, etc. De diverses façons il contribue à hausser la productivité des équipes de travail : il évite des déplacements, raccourcit les délais de consultation entre les échelons de l'organisation, offre des fonctions de messagerie et permet le travail en groupe, même entre participants éloignés.

Pour le dirigeant, l'Intranet est un outil inégalé de communication, d'information, de gestion, parfois à distance, des documents, du savoir et de la production de l'entreprise. $L$ 'Intranet met les données sensibles de l'entreprise à l'abri de la curiosité des concurrents. L'effort (pécuniaire et autre) nécessaire pour actualiser, par l'adoption d'un Intranet, l'infrastructure numérique de la firme, dote les usagers d'un système efficace qui permet, notamment, de sécuriser le serveur du réseau, d'optimiser la performance de l'équipement informatique et d'en assurer la gérance au quotidien.

Un dirigeant qui a vu les avantages d'un Intranet voudra s'en pourvoir dès que possible. Il verra en même temps la nécessité de modifier ế de renforcer son parc informatique. Survient alors un choix à faire: s'équiper pour les besoins présents ou, considérant l'évolution 
constante des TIC, investir davantage maintenant dans un système puissant capable de répondre à des besoins futurs prévisibles, ou qui pourra être rehaussé éventuellement selon les circonstances.

Après consultations des pairs, des foumisseurs, cette décision revient au dirigeant (avec la petite équipe qui l'entoure), qui a à évaluer les cô̂ts et les avantages, et à supputer le retour sur son investissement. Quoi qu'il en soit, l'installation d'un Intranet est un pas décisif pour une entreprise, qui y voit, outre les avantages propres à ce réseau, l'occasion de hausser les performances de ses matériels informatiques et, en bout de ligne, d'améliorer sa gestion et sa productivité.

Par ailleurs, les entreprises qui comptent sur les services de conseillers et de fournisseurs en materiel et en logiciels ont tendance à accélérer l'implantation d'un matériel informatique sophistiqué; alors que le recours aux fournisseurs d'Internet freine plutôt cette implantation.

La réticence des dirigeants d'entreprises envers les fournisseurs d'Internet peut s'expliquer par la contradiction qui semble exister, dans le cas des territoires périphériques de notre étude, entre le discours de ces fournisseurs et la réalité vécue par les entreprises. Le discours des fournisseurs d'Internet vante les avantages de ce média, tel qu'il est offert dans les métropoles, qui profitent de l'Internet à haute vitesse; alors que ce service n'est pas encore disponible dans tous les territoires périphériques. Cette contradiction infirme les arguments des fournisseurs d'Internet. Par contre, le soutien des conseillers et des fournisseurs en matériel informatique et logiciels permet aux dirigeants de trouver les solutions recherchées, car ces conseillers adaptent les produits technologiques aux besoins de leurs clients et aux infrastructures en place. Leur soutien semble, en fait, essentiel pour mieux identifier les besoins technologiques de l'entreprise et pour choisir le matériel et les logiciels qui répondent adéquatement aux besoins.

Il ressort des multiples visites que nous avons faites sur le terrain que les chefs d'entreprises sont généralement conscients de l'importance de disposer d'outils informatiques technologiquement avancés, mais qu'ils n'ont ni le temps ni le goût de se plonger dans l'étude et la comparaison des divers systèmes offerts sur le marché. C'est pourquoi ìs s'en remettent à des conseillers expérimentés et à des foumisseurs de confiance. Ceux-ci par contre ont 
intérêt à proposer des solutions qui répondent non seulement aux besoins présents, mais adaptables à des conditions qui peuvent changer. 11 importe aussi que ces solutions soient conviviales et facilitent les rapports entre l'entreprise et ses interlocuteurs. Par exemple, lorsqu'il s'agit de passer une commande, de spécifier un article chez un grossiste ou un marchand de matière première, il faut que les équipements informatiques soient compatibles, que les codes d'articles soient identiques, etc.

Grâce aux TIC, notamment l'ordinateur portable et le téléphone cellulaire, le dirigeant n'est plus rivé à son bureau; il peut consacrer du temps à l'entretien de relations avec ses pairs, parfois à quelque loisir. Bref, pour la suite de l'entreprise, ces personnes qui gravitent autour du secteur numérique ont une grande influence.

Par ailleurs, notre analyse a montré que la participation des dirigeants d'entreprises à des activités extérieures et leur affiliation à un réseau d'entreprises semblent avoir peu d'incidence sur l'appropriation du matériel informatique, mais encouragent fortement l'appropriation des logiciels. Ce constat peut s'expliquer par l'écart qui s'établit dans une économie d'information entre l'action individuelle et ses dimensions collectives. C'est qu'un dirigeant prend seul la décision d'implanter ou non les composantes d'un système informatique. Mais une fois cette étape franchie, c'est le cadre général dans lequel il agit qui lui suggère les utilisations pratiques, donc logicielles, qui lui sont offertes. C'est dire que l'appropriation des logiciels se définit souvent comme une réponse rapide et précise à un besoin immédiat.

L'affiliation à un réseau semble donc devenir un choix important, qui permet au dirigeant qui a déjà implanté son matériel informatique de s'informer des caractéristiques de divers logiciels et de leur capacité à améliorer le fonctionnement de son entreprise. Le cadre délimité par les réseaux d'entreprises et par les diverses activités sociales sert donc à catalyser l'appropriation des logiciels. Comme nous l'avons constaté chez certaines entreprises des territoires périphériques, la rationalisation des opérations est d'autant plus intéressante lorsque les systèmes logiciels, et les systèmes d'information en particulier, permettent de réaliser des gains de productivité et d'améliorer la qualité globale des systèmes de gestion. Les modèles de gestion ainsi constitués apportent en effet des solutions inédites à de nombreux problèmes structuraux. Cependant, et par la nature même des systèmes informatiques et d'information, 
les solutions apportées ne peuvent être ni définitives ni radicales. Le dirigeant est donc constamment invité à les modifier, à les améliorer, en même temps qu'il améliore ses systèmes informatiques et qu'il s'adapte aux circonstances. Il doit donc, chaque fois, établir ses véritables besoins, juger, après consultations s'il y a lieu, de l'opportunité d'y pourvoir, puis décider.

Jusqu'ici et dans toutes les situations analysées, le facteur régional ne semble pas être important, car le territoire d'implantation, qu'il soit métropolitain ou périphérique, n'influence pas pour autant les pratiques d'une entreprise se dotant d'une stratégie technologique. Il faut plutôt voir là une situation nouvelle, où les entreprises des territoires périphériques peuvent semblablement tirer profit des technologies de pointe puisque, sous ce rapport, elles ont le même accès aux avancées technologiques que leurs homologues d'un territoire métropolizain.

En somme, quand on considère selon diverses variables l'appropriation du matériel informatique et des logiciels, il apparait que les possibilités d'accès sont semblables dans nos quatre territoires, sauf pour Internet à haute vitesse dans les territoires périphériques. Toutefois cette situation est en voie d'être corrigée. Il apparaît également que cette appropriation est plus avancée dans la région de Québec, bien que des efforts importants soient faits dans les territoires périphériques. Ce retard a sans doute plus d'une cause, et l'on comprend que les conditions nécessaires au rattrapage de ces territoires ne dépendent pas uniquement des acteurs qui y sont installés; elles les dépassent.

\subsubsection{Les effets de la taille d'une entreprise}

Il importait de chercher à comprendre les effets de la taille d'une entreprise sur ses pratiques, sur son aptitude à s'approprier des objets et des outils technologiques, de même que sur le fonctionnement d'une économie d'information. Dans la région de Québec, une sorte de stagnation a été constatée chez un grand nombre d'entreprises. Généralement, celles-ci ont des relations d'affaires bien établies, soit avec des entreprises implantées dans une autre métropole, généralement à Montréal, soit avec des instances gouvernementales ou des grandes entreprises locales avec qui les dirigeants de ces PME maintiennent des relations privilégiées. Il s'en suit qu'un grand nombre de ces PME ne font que de la sous-traitance, 
n'exécutant que des tâches conçues par leurs partenaires d'affaires, ce qui paralyse linnovation.

Principalement dans ce territoire (Québec), les entreprises qui sont bien établies et qui jouent souvent un rôle important dans les dynamiques en place semblent peu inquiètes de l'avenir et peu enclines à s'efforcer d'innover. Les structures en place, de même que l'appui qu'elles trouvent chez leurs partenaires traditionnels (les CLD, les chambres de commerce et les autres organismes publics et parapublics) et dans leurs réseaux d'affaires, les confortent dans leur position. En fait, les discussions que nous avons eues dans ce territoire donnent à penser que les rapports sécurisants que des entreprises entretiennent avec leurs clients et leurs fournisseurs auraient une influence plutôt négative sur leur souci de diversifier leurs pratiques. Les entreprises se préoccupent d'abord de stabilité. Elles se soucient davantage de la fiabilité de leurs partenaires, canaux pour la commercialisation de leurs productions, que des ouvertures et des fluctuations des marchés. La plupart des entreprises, petites ou moyennes, confient leur production à des intermédiaires bien installés dans une métropole, qui se chargent de l'écouler sur les marchés national et international. On comprend donc pourquoi les entreprises se préoccupent insuffisamment de productivité et de compétitivité et d'innovation.

Par contre, certaines entreprises de petite taille implantées dans les territoires périphériques se distinguent par une synergie qui favorise l'échange intensif de l'information, des connaissances et des points de vue, au sein de l'entreprise et entre elles. Les échanges amènent le dirigeant à mieux apprécier l'utilité, même la nécessité d'actualiser ses installations de communication, de gestion et d'information. Les rapports humains et sociaux traduits par des liens de confiance et de solidarité qui se nouent entre les employés et souvent avec les dirigeants, sont davantage enracinés et constituent une énergie convergeant vers le progrès de l'entreprise. Pour cette raison, nous avons constaté que les petites entreprises, en raison de leur volonté d'automatiser la gestion et le fonctionnement de leurs systèmes d'information, sont davantage intéressées à adopter des procédés de traitement et de diffusion de l'information par de nouveaux moyens.

Les dirigeants de ces entreprises montrent en effet un enthousiasme extraordinaire, qui les incite à évaluer régulièrement les techniques et les applications déjà en place. C'est pourquoi 
les petites entreprises tendent à agir non seulement sur le fonctionnement de leurs propres systèmes, mais aussi à diversifier leurs sources d'information. Elles s'emploient à alléger les contraintes, à faire évoluer les attitudes, dans un environnement dominé par des entreprises bien établies, mais moins motivées à se remettre en question.

Par ailleurs, les entreprises des territoires étudiés exploitent presque de la même manière le courriel; ce moyen de communication rendant partout les mêmes services, il s'avère utile pour tout le monde. Cette popularité montre bien l'engagement des entreprises dans l'ère numérique. Toutefois, lorsqu'un dirigeant utilise le courriel, il a à considérer ses aptitudes à informer et à communiquer par écrit. Contrairement à la communication orale, l'écrit suppose la maitrise de la grammaire et de l'orthographe, pour la clarté et la compréhension du message, autant pour convaincre que pour donner une bonne image de soi et de l'organisation. D'autant plus que le courriel demeure un document qui peut être archivé, utilisé à différentes occasions et comme preuve officielle. Ce sont donc de nouvelles exigences qui modifient les tâches de direction, mais qui, à terme, enrichissent les communications.

L'évolution des pratiques entrepreneuriales montre donc une amélioration notable du savoir et du savoir-faire des dirigeants. L'amélioration touche les entreprises de tous les territoires. Ainsi, quand nous nous sommes intéressé aux effets de la taille d'une entreprise sur le choix des moyens internes de communication, nous n'avons rapporté aucune différence significative entre les territoires étudiés, sauf pour l'Intranet, qui est plus utilisé à Québec. Les entreprises d'aujourd'hui, quelle que soit leur taille et leur milieu d'implantation, savent l'utilité des communications internes et optent pour le moyen le plus apte à remplir cette fonction. Qu'il soit basé sur une technologie de pointe ou non, l'essentiel est que l'information puisse circuler entre la direction et le personnel et, au besoin, entre les employés.

Quant à l'Intranet, son usage est légèrement plus répondu en territoire métropolitain, mais cette différence n'est pas suffisamment importante pour justifier un écart dans les dynamiques économiques. Pour une firme de petite taille, il n'est pas dit qu'un tel réseau interne soit toujours nécessaire. Par ailleurs, comparativement aux autres moyens de communication interne, l'implantation d'un Intranet est souvent coûteuse, et sa gestion exige des ressources financières et techniques qui ne sont pas toujours à la portée des PME implantées dans un 
territoire périphérique. Alors que dans une métropole, où une main-d'œuvre qualifiée se trouve plus facilement, les entreprises semblent prendre plus de risque et d'initiative. Le fonctionnement même d'un Intranet est une retombée d'une culture davantage ouverte, propre aux centres urbains, où l'entrepreneur doit protéger l'information qui circule et les directives qu'il adresse à son personnel et à ses équipes de travail. L'utilisation d'un Intranet n'exige-telle pas une authentification, un mot de passe ainsi que des coupe-feu ? Au-delà de la commodité qu'apporte à l'entreprise un Intranet, le dirigeant y trouve un excellent moyen de contrôler son espace, de démarquer les zones, quant à la régie interne de l'entreprise et quant aux communications extérieures. Les employés d'une telle organisation peuvent se sentir privilégiés car ils reçoivent une information personnalisée et dont la diffusion est limitée, ce qui tend à développer le sentiment d'appartenance à cette organisation. Celle-ci toutefois peut se trouver dans une situation quil l'empêche de s'ouvrir convenablement sur son milieu.

Ce que nous avons constaté, c'est que'en général, les effets des TIC sur les pratiques entrepreneuriales ne varient pas en fonction des territoires et de leurs dynamiques, mais en fonction des aptitudes des entrepreneurs à les intégrer à leurs pratiques quotidiennes. Il est évident que, dans une économie d'information, les dirigeants adoptent de la même façon le courriel, l'Intranet, etc., et se voient dans l'obligation d'adopter de nouvelles façons de gérer les archives et de traiter l'information. Certaines entreprises, surtout en milieu urbain, vont adopter une stratégie globale et efficace de gestion électronique de documents et d'information. Par exemple, parmi les entreprises que nous avons visitées à Québec, certaines ont déjà implanté des solutions informatiques novatrices, mais pour plusieurs le chemin à parcourir est encore long et nécessite l'acquisition d'un nouveau savoir-faire.

Il semble donc que, indépendamment de son territoire d'implantation ou de sa taille, une entreprise réagit généralement de la même façon par rapport aux technologies. On voit là une stratégie de rattrapage des territoires périphériques, car les entreprises y adoptent les mêmes pratiques que celles de leurs homologues implantées dans un territoire métropolitain. Le changement technologique ambiant ne peut qu'influencer les dirigeants d'entreprises, quel que soit leur lieu d'implantation, et les mêmes comportements tendent à se généraliser. 


\subsubsection{Le commerce électronique ế l'exploitation du potentiel du Web}

L'appropriation et l'usage des TIC contribuent au rattrapage des territoires périphériques en y apportant des pratiques et des attitudes naguère caractéristiques des seuls territoires métropolitains, surtout en ce qui conceme le commerce électronique et l'exploitation du potentiel du Web. Ainsi, nous avons constaté que le degré d'utilisation d'Internet par les entreprises implantées dans les quatre territoires a des effets importants sur le recours des entreprises au commerce électronique. Partout, l'usage de l'Internet pour établir ou maintenir des liens avec les foumisseurs, et son usage à des fins personnelles, favorisent le recours au commerce électronique. II n'existe pas à cet égard de différence significative entre les comportements des entreprises implantées dans les quatre territoires concernés. Un dirigeant d'entreprise qui utilise le Web pour s'ouvrir sur le monde élargit ses horizons et tend à lui faire confiance pour y faire des transactions. S'ouvrir sur le monde, c'est aussi s'aventurer, être disposé à faire autrement.

Il apparaît de plus que l'usage de l'Internet aux fins de communication interne et de gestion favorise l'exploitation du potentiel du Web, cet usage menant à la découverte de méthodes nouvelles de s'informer, d'administrer et de transiger. C'est dans les entreprises de la CUQ, par rapport aux trois autres territoires, que l'on exploite le mieux les possibilités de la Toile, mais partout on pourrait faire davantage.

Les TIC offrent aux entrepreneurs un champ d'exploration illimité. Quel que soit leur territoire d'opération, les entreprises ont jusqu'ici convenablement intégré les systèmes informationnels, mais abordent avec circonspection les transactions électroniques et les autres possibilités qu'offre le Web.

\subsubsection{Des aspects négatifs à l'informatisation?}

La littérature afférente à l'utilisation des outils informatiques voit généralement d'un oeil approbateur, souvent émerveillé, les avantages qui en découlent, et a peu à dire sur les inconvénients possibles. Le présent texte ne fait pas exception. Pourtant, il est rare que l'introduction d'une technologie nouvelle ne comporte pas un quelconque aspect négatif. Notre analyse en a relevé quelques uns, qui accompagnent à l'occasion l'implantation ou 
l'usage des TIC. Il y a d'abord les coûts d'implantation à considérer. Une petite entreprise peut ordinairement se permettre les outils de base : ordinateur, quelques logiciels, courriel, etc. Mais pour la technologie de pointe, les coûts d'achat, d'installation et de maintenance technique peuvent être prohibitifs et l'accès réservé de facto aux firmes de taille assez considérable. L'appropriation d'une nouvelle technique ou d'une technologie requiert de plus un effort intellectuel qui peut être important et repose ordinairement sur d'autres compétences acquises préalablement.

Il faut compter également sur un phénomène normal dans toutes les organisations: la résistance au changement. Le changement n'est pas nécessairement bon partout; c'est pourquoi il peut susciter de l'insécurité et de la résistance, non seulement chez les employés, mais aussi chez les cadres, qui peuvent entrevoir le risque d'être dépossédés de leur utilité. II importe en conséquence de prendre le temps d'établir une stratégie adéquate. Parfois, quand le fonctionnement de l'entreprise s'y prête, une expérience pilote peut être tentée.

\subsubsection{L'entreprise et son milieu}

Une entreprise existe dans un lieu physique, le territoire; les dirigeants et le personnel de cette entreprise font partie d'une communauté, d'un groupe social. Notre analyse a fait ressortir quelques constatations sur l'influence que pourrait avoir le territoire sur l'appropriation des nouvelles technologies par l'entreprise; des constatations également sur l'effet social de l'appropriation des TIC.

\subsubsection{Le facteur régional}

Sous tous les aspects considérés, notre étude montre que des différences significatives, autres que celles relatives aux ressources et aux infrastructures, sont à peu près inexistantes entre les territoires. Néanmoins la géographie demeure la même; un territoire périphérique va le demeurer, mais il n'est pas de ce fait acculé à la stagnation. Nous avons vu plus haut dans quelle direction certains aspects de la pratique entrepreneuriale pourraient être infléchis et tonifiés. Les technologies numériques sont désormais l'atout majeur dont disposent les territoires périphériques pour opérer leur rattrapage. Pour un nombre toujours plus grand de fonctions: interventions, négociations d'affaires, besoins de tout genre, qui exigeaient naguère temps et déplacement, les nouvelles technologies abolissent le temps et l'espace et accroissent 
la productivité. En fait même les petites entreprises ont réussi à réduire l'écart qui les séparait des grandes, quant aux technologies de base. Mais très souvent le manque de ressources financières empêche d'aller plus loin.

La situation géographiquement excentrique d'un territoire n'est pas nécessairement un obstacle prohibitif au développement. La Finlande en donne un exemple instructif, où on a réussi à obvier en même temps à deux obstacles majeurs: la distance et le froid. Dans ce pays, la Laponie se trouve au cercle polaire, plus éloignée de la capitale Helsinki que l'est Manicouagan de Montréal. Avec de la vision, de la concertation et de la volonté politique, on a procuré à cette région un développement équitable et un dynamisme économique comparable à celui des autres parties du pays, en la dotant d'infrastructures de transport et de télécommunications à la hauteur des besoins, et en y rendant les mêmes services qu'ailleurs. C'est dire que la présence d'infrastructures et de services de qualité dans un territoire périphérique est une condition de sa compétitivité.

\subsubsection{Les TIC induisent des changements sociaux}

L'influence croissance des TIC dans tous les domaines sollicite non seulement les acteurs de développement, mais la société tout entière. Bien que notre étude n'ait pas eu pour but d'évaluer l'effet social de la technologie numérique, il ne parait pas inopportun de s'attarder un moment sur cette question. Une sorte de dialectique s'est établie quant à l'utilisation des outils informatiques. Elle a fait évoluer la logique mercantile de la production et du profit, vers une logique à contenu social, qui amène les utilisateurs à élargir leurs champs d'interaction et à s'avancer dans des formes nouvelles de sociabilité.

Nées modestement dans des laboratoires de recherche, les TIC se sont étendues comme un halo autour d'une brillante source lumineuse. En moins de quinze ans, elles sont devenues indispensables à la science, au commerce, à l'industrie et à combien d'autres domaines. En même temps, irréversiblement elles ont pénétré toutes les couches de la société et ont influencé les pratiques individuelles et collectives. Les attitudes évoluent, de nouveaux comportements apparaissent, indices de l'intégration des TIC à la vie quotidienne. Parmi la multiplicité des usages, les plus voyants sont sans doute le courriel, le clavardage, la 
navigation dans le cyberespace à la recherche de renseignements sur à peu près tous sujets concevables.

Parce que le monde du travail est le plus directement touché, lordre social qui se met en place tend à valoriser les métiers et les professions qui exigent un haut degré de savoir, conscient que le savoir est source de progrès et de richesse. C'est ce que suggère notre analyse: plus les acteurs recourent à un matériel informatique varié et à des logiciels astucieux, plus ils sont habiles à exploiter des savoirs diversifiés et complexes.

Il semble que le recours à une technologie nouvelle a sensiblement les mêmes effets partout; il y a inévitablement un certain débordement technique et des retombées sociales qui se concrétisent en applications inédites et en comportements nouveaux. Les interactions entre acteurs et avec leur milieu, leur implication dans la vie communautaire, les invitent à mettre le développement technique non seulement au service de leurs activités professionnelles et sociales, mais aussi à en faire profiter la collectivité de façon ou d'autre. La technologie contribue de la sorte à dessiner la matrice d'une régulation institutionnelle et donc sociale toujours en construction.

En somme, l'appropriation des TIC ne profite pas qu'aux entreprises; la société peut également en tirer avantage. Des liens de communication et d'interaction se tissent, indépendants de la distance, que les moyens usuels n'auraient pas permis. C'est donc le substrat d'une certaine identité territoriale, soutien de la singularité d'une communauté, qui tend à s'agrandir. Il semble qu'une meilleure connaissance de l'Autre, de l'Ailleurs, devrait favoriser plus de collaboration et moins de confrontation. L'avenir montrera sans doute des effets positifs de cette évolution et, il faut l'espérer, peu de conséquences indésirées.

\subsection{Perspectives}

S'il est juste de considérer que la mâtrise des outils informatiques est désormais indispensable au succès d'une entreprise, la présente étude montre que cette acquisition est bien enclenchée. Malgré l'inquiétude et les pertes, entrainées par l'effondrement de la bulle technologique qui survenait au moment de la collecte de nos données, l'acquisition et l'usage des technologies de linformation et de la communication ont paru grandissants, notamment dans deux domaines 
vitaux de l'entreprise: la circulation de l'information et linnovation technologique. Notre étude montre que les TIC fournissent aux entreprises un apport important, particulièrement pour certains usages. Elle révèle aussi des possibilités à explorer davantage.

\subsubsection{Apport des TIC à l'entreprise}

La puissance et la commodité des outils informatiques, et les avantages qu'une entreprise diligente peut en retirer, ont été décrits précédemment. Notre analyse indique que ces mêmes avantages ne sont pas inaccessibles, même en territoire périphérique; que les TIC s'intègrent aisément dans tous les territoires; que partout on les utilise, bien qu'à divers degrés, à des fins variées.

\subsubsection{Les besoins informationnels}

Par rapport à l'usage des TIC, les entreprises cherchent surtout à se renseigner sur l'appropriation des diverses composantes et sur les banques de données. L'information de caractère général recherchée concerne principalement les nouveaux produits, la concurrence, Ia technologie et la gestion. Quant aux moyens d'interaction servant à recevoir ou transmettre l'information, les moyens classiques (notamment le téléphone et le télécopieur) continuent de jouer un rôle important, en même temps que le courriel gagne du terrain, de même que les rencontres personnelles.

Par ailleurs, la réunion du personnel, le tableau de nouvelles et le bulletin interne sont des moyens de communication interne qui influencent toujours les pratiques entrepreneuriales. On note avec intérêt toutefois ce que l'analyse a fait ressortir: les entreprises qui utilisent le bulletin interne et l'Intranet comme moyens de communication s'approprient davantage les outils informatiques.

Parmi les activités extérieures, les repas, les activités caritatives et les activités de reconnaissance accaparent la plus grande partie des activités des dirigeants hors de leur milieu de travail. Les occasions de repas sont, par nature, beaucoup plus fréquentes que, par exemple, la tenue d'expositions. La participation à des réseaux d'entreprises ou interorganisationnels est un autre aspect, plus formel, de l'activité extérieure, qui semble favoriser 
l'appropriation du matériel informatique et des logiciels. Les entreprises qui sont affiliées à un réseau ont tendance à mieux s'approprier un plus grand nombre de logiciels.

\subsubsection{L'innovation technologique}

Certaines pratiques sont de nature à encourager l'adoption de technologies innovantes. L'une d'elles est la recherche de soutien extérieur pour renforcer la capacité de l'entreprise en technologie de l'information et de la communication. De plus, les conseillers et fournisseurs de composantes informatiques, de même que les foumisseurs d'accès à Internet et les Webmestres, influencent largement l'appropriation du matériel informatique et des logiciels. Le recours aux conseillers et fournisseurs se traduit par une implantation plus rapide et mieux réussie du matériel informatique et des logiciels.

Le branchement à Internet est un premier pas en vue de s'ouvrir au monde extérieur. L'utlisation d'Internet varie selon les entreprises, mais de façon générale ce réseau sert d'abord pour le courriel. Viennent ensuite l'acquisition de données stratégiques, les liaisons avec les fournisseurs, le repérage de nouveaux contacts. Cet usage semble encourager les entreprises à s'approprier le matériel informatique et les logiciels. Pour l'entrepreneur, la fréquentation d'Internet est une invitation à utiliser le potentiel considérable du Web pour fins commerciales. Les entreprises qui y font des achats pourraient exploiter davantage le potentiel de la Toile. Nous avons constaté que l'usage de la Toile s'accroît avec les communications internes et de gestion, les liaisons avec les fournisseurs et l'usage personnel. Il est aussi important pour l'acquisition de données.

Dans pratiquement toutes les entreprises, au moins les éléments de base des TIC sont

disponibles et utilisés, que ce soit les matériels ou les logiciels. Pour les entreprises qui ne possèdent pas d'équipement très sophistiqué, il s'agit peut-être d'une question de coût ou que leur type d'activité ne le requiert pas.

Le matériel informatique est fortement standardisé, ce qui encourage naturellement l'uniformisation des utilisations. Les logiciels toutefois sont beaucoup plus diversifiés et spécialisés, surtout dans la région de Québec. C'est sans doute que la nature même de certains logiciels spécialisés, conçus pour répondre à des besoins bien circonscrits, correspond bien à 
la réalisation de tâches associées au commerce, traditionnellement exercées surtout dans les villes.

\subsubsection{Des possibilités à explorer davantage}

Sous plusieurs aspects les territoires périphériques ont du rattrapage à faire, et apparaissent des avenues de développement qu'il y aurait lieu d'explorer attentivement. Chacun à leur manière, les entrepreneurs et les pouvoirs publics auraient intérêt à s'y engager, si l'on veut offrir à ces territoires des possibilités de progresser qui puissent se comparer avec celles des territoires métropolitains.

\subsubsection{Pour l'entreprise: des orientations à considérer}

Comme ce fut le cas pour les innovations du passé qui ont duré, celles d'aujourd'hui ne sont pas adoptées inconsidérément. Elles doivent avoir une fonction clairement perceptible, que ce soit pour le divertissement, le transport, le travail, etc. et améliorer ce qui existait déjà. Pour l'acteur de développement, une nouvelle technologie s'imposera et sera adoptée si elle lui parait avantageuse pour ses affaires, si le rapport coût-avantage s'annonce favorable et si, le cas échéant, elle s'est avérée profitable ailleurs.

C'est pourquoi il importe que le dirigeant d'entreprise se tienne constamment au fait de la conjoncture, de la concurrence, de la réglementation, de l'évolution technologique. Notre analyse laisse voir, à cet égard, que le dirigeant aurait tout avantage à diversifier ses sources d'information. Les centres de recherche, les universités, les sociétés de développement et autres organismes détenteurs de savoir doivent être consultés davantage. Les entrepreneurs doivent miser plus sur les visites d'entreprises canadiennes et étrangères et la consultation des services gouvernementaux, surtout fédéraux, qui sont négligés. Les publications, spécialisées ou non, pourraient servir davantage. Également, un entrepreneur a intérêt à diversifier ses activités extérieures, particulièrement à intensifier sa participation à des réseaux d'entreprises. L'appropriation des outils informatiques encourage l'affiliation et celle-ci, une meilleure appropriation, les deux contribuant à mettre en valeur le potentiel collectif dont disposent les acteurs. 
Dans les territoires étudiés, on utilise les TIC à des fins variées, mais de façon inégale. Les acteurs pourraient les utiliser davantage et de façon plus profitable; l'appropriation de la technologie numérique encourage la multiplication de ses applications et inversement les applications favorisent l'appropriation.

\subsubsection{L'appui des pouvoirs publics}

II est́ évidemment souhaitable que le Québec dispose de pôles économiques importants et dynamiques. Pour de multiples raisons, d'ordre économique, démographique, social, les pouvoirs publics ont la responsabilité de veiller aussi au développement des territoires dits excentriques et de rendre possible à leurs populations un niveau de vie convenable. Les entreprises, de toutes tailles, sont le moteur économique et social des régions. Elles ont des besoins, que le discours officiel reconnait, trop souvent sans accompagner ce discours de mesures concrètes. Ces besoins sont variés et appellent à la concertation de différents paliers gouvernementaux.

Pour un pays, l'occupation du territoire est un impératif économique autant que politique, ce qui exige des pouvoirs publics de tenir compte des changements, technologiques et autres, qui tendent à déstabiliser les territoires périphériques et portent la population à quitter vers les grands centres. Les entreprises des territoires métropolitains profitent de structures déjà en place, qu'on entretient et améliore au besoin; le cadre social diversifié favorise la circulation de l'information et l'interaction. Dans les territoires périphériques, les desiderata sont visibles et perdurent; le seul sentiment d'appartenance ne saurait y satisfaire.

Dans chaque territoire il serait à propos de renforcer un cadre institutionnel qui aide les acteurs à mieux s'informer, à profiter de linformation stratégique porteuse de productivité, à rechercher la concertation. L'acteur isolé se prive d'appuis précieux, particulièrement du soutien de ses pairs. C'est pourquoi l'affiliation à des réseaux devrait être vivement encouragée. Il faudrait de plus convaincre les entreprises des avantages de l'usage du Web pour le commerce électronique et comme vitrine pour leur production.

De nos contacts avec les dirigeants d'entreprise, nous avons acquis l'impression d'une certaine méconnaissance des possibilités des $\mathrm{TIC}$, non seulement chez certains gestionnaires, mais aussi au sein des organismes ayant mandat de les aider. Des entrepreneurs se sont 
équipés d'ordinateurs, d'imprimantes ou d'autres composantes, qu'on trouve bien commodes pour le traitement de texte, la comptabilité, éventuellement le courriel, sans chercher à exploiter davantage les possibilités de l'informatique. On est industrieux, débrouillard, mais il arrive qu'on soit tellement appliqué à la gestion des problèmes quotidiens, que le temps qu'il faudrait pour prendre du recul, étudier de meilleures façons de faire, innover, ne vient jamais. Il faut aussi, bien sûr, pour tirer pleinement parti des outils informatiques, que l'infrastructure technologique soit en place et que les ressources intellectuelles nécessaires soient disponibles.

Quant aux organismes d'aide à l'entreprise, des entrepreneurs sont d'avis que leur utilité, non négligeable, pourrait s'accrôtre. Dans un territoire existent divers organismes d'aide, de niveau local, provincial ou fédéral. On considère que trop souvent ces organismes ne réussissent pas à dépasser leurs enjeux politico-administratifs; qu'ils pourraient se concentrer moins sur le soutien technique et financier et davantage à l'étude des véritables besoins de l'entreprise, sur leur rôle de décodeurs entre des sources d'information complexe et une réceptivité inégale chez les entrepreneurs. On voudrait aussi que ces organismes se fassent plus rassembleurs de façon à favoriser le réseautage. Bref, on souhaite que ces organismes se "parlent" davantage, qu'ils se concertent, que les intervenants sortent plus souvent de leurs bureaux pour aller sur le terrain rencontrer ceux qui font tourner la roue économique d'un territoire. Idéalement, les chefs d'entreprises voudraient bien pouvoir s'adresser à un guichet unique pour leurs besoins, car parmi des organismes aux vocations similaires, l'effort nécessaire pour trouver le service approprié en rebute plusieurs. Pour nous, il parait indispensable que ce guichet unique soit orienté vers la promotion des technologies de pointe.

\subsubsection{Horizons technologiques des territoires étudiés}

Nos résultats montrent que l'on utilise les TIC pour diverses fins: entretenir des rapports sociaux, s'informer, acheter, vendre, s'afficher, etc. Cet usage enrichit les dynamiques de la vie d'une collectivité, métropolitaine ou périphérique, et ouvre de nouveaux horizons devant les acteurs individuels et devant les territoires.

La tranjectoire est presque identique dans les territoires étudiés et cette similitude s'expliquerait, selon notre analyse, par une aptitude semblable des acteurs à apprécier les avantages des TIC et à les intégrer à leur savoir-faire. Cela a des conséquences significatives 
sur les dynamiques économiques et sociales, tributaires de l'aptitude des acteurs et des territoires à se doter des stratégies idoines de développement.

La participation des composantes technologiques à ces dynamiques dépend du talent des acteurs à bien les intégrer à leurs stratégies d'action. On peut donc postuler que l'appropriation des technologies contribue à façonner l'avenir des territoires, mais que l'héritage de ces territoires peut freiner le développement. Le tableau suivant, que nous avons élaboré à la lumière des résultats de notre analyse, permet de cerner la participation de diverses variables à l'enrichissement et à la diversification des dynamiques d'un territoire.

Tableau 10 : Caractéristiques liées à l'usage des IIC

\begin{tabular}{|l|l|l|l|l|}
\hline & Standardisation & Diversitéé & Valeur ajoutée & Ressources \\
\hline Matériel & Forte & Faible & Variable & Complémentaires \\
\hline Logiciel & Faible & Forte & Haute & Puissantes \\
\hline Réseaux & Variable & Forte & Variable & Puissantes \\
\hline Organisations & Faible & Forte & Forte & Supplémentaires \\
\hline Société & Variable & Forte & Variable & Variables \\
\hline
\end{tabular}

Cette classification montre que le matériel informatique, qui comprend les technologies classiques (le téléphone, la télécopie) et les technologies de pointe (ordinateur, Internet, etc.), se distingue par sa grande standardisation, les objets qui constituent ce matériel n'encourageant pas une utilisation diversifiée. Bien que ce matériel ajoute de la valeur aux différentes activités économiques et sociales, cette valeur dépend largement du contexte économique et social ambiant, de l'héritage, mais aussi de la capacité des acteurs individuels et collectifs à en profiter pour travailler à des initiatives innovantes. Comme le montre notre analyse, un usage convenable du matériel informatique favorise l'exploitation complémentaire des objets traditionnels et d'autres qui relèvent de la haute technologie. Cette tendance est commune aux territoires étudiés. On ne trouve aucune différence significative entre les territoires.

Il en va autrement pour les logiciels. Ils sont utilisés davantage en territoire métropolitain que dans les territoires périphériques; c'est le cas principalement de l'Intranet. Cette situation peut 
s'expliquer par la nature même des logiciels, qui servent à la réalisation de tâches d'origine surtout urbaine: vente, achats, commerce, etc. Dans le contexte actuel, la conception et le développement des logiciels se font au rythme des innovations dans les activités économiques et sociales. Celles-ci étant plus denses en un milieu métropolitain, les logiciels varient beaucoup et cette diversité restreint la standardisation des pratiques. Vu qu'ils sont ordinairement conçus pour répondre à des besoins immédiats et bien précis, l'usage de ces logiciels ajoute une valeur inestimable aux activités qu'ils intègrent et constituent ainsi des ressources supplémentaires et puissantes pour qui sait s'en servir convenablement.

Notre analyse indique que l'informatisation des entreprises encourage le réseautage, qui devient alors un instrument de mise en valeur collective des ressources dont disposent les acteurs d'un territoire. Comme on peut voir au tableau qui précède, les circonstances, les objectifs des acteurs amènent une grande diversité dans les réseaux, mais pour leurs participants, tous offrent un potentiel de progrès qui peut être considérable.

Le tableau montre par ailleurs que l'instauration des TIC s'est accompagnée d'une faible standardisation des configurations organisationnelles. En effet les PME s'approprient la technologie chacune à sa façon et selon ses besoins; elles adoptent diverses stratégies dont les effets dépendent de l'adéquation entre la structure adoptée pour l'organisation et les objectifs poursuivis. Les TIC n'ajoutent donc de la valeur aux activités d'une PME que dans la mesure où les solutions technologiques constituent une ressource supplémentaire. Bien encadrée, cette ressource, et les changements organisationnels qu'elle induit, peut s'avérer de grande valeur pour l'entrperise.

En définitive, c'est la société qui se trouve influencée par l'usage des TIC. Cette influence est évidemment variable, comme l'indique notre tableau. Elle varie selon le moment, le milieu. Elle peut être d'autant plus considérable que l'appropriation est mieux réussie et que des effets favorables se font sentir sur les conditions de vie et de travail. On assiste donc à un renouvellement des pratiques sociales, non seulement dans le monde des affaires, mais dans tous les champs d'activité.

L'arrivée massive des TIC, et les changements dans la conjoncture qui l'accompagnent, imposent l'exploration de nouveau repères sociaux, des adaptations individuelles et des 
ajustements structurels, au regard de la diversité des horizons technologiques ouverts devant les acteurs et les territoires. Cette diversité n'offre pas pour autant une démarche particulière et uniforme à suivre. Les dynamiques économiques et sociales de développement d'un territoire sont constamment confrontées à des contraintes de tous ordres. La généralisation du savoir, des connaissances scientifiques et de l'information stratégique oppose les anciennes pratiques aux exigences des structures qui s'universalisent. L'ouverture des territoires périphériques sur un nouveau monde technologique engendre des perspectives nouvelles devant les communautés qui veulent se prendre en charge, en fondant leurs initiatives de développement sur des bases scientifiques.

On considère généralement que la vitalité économique et sociale d'une région dépend avant tout du dynamisme des entreprises qui s'y trouvent. Au cours de cette recherche, nous avons examiné et tenté de jauger, selon certains critères, le dynamisme économique des entreprises de quelques territoires du Québec. L'appropriation des TIC, les effets de la taille d'une entreprise et son aptitude à intégrer l'économie d'information ont principalement orienté notre réflexion. Et nous est apparue une conclusion que nous croyons importante: que vu sous l'angle d'une économie d'information, l'écart entre le dynamisme d'un territoire périphérique par rapport à celui d'une métropole tend à se rétrécir.

Il apparaît par ailleurs que si un écart persiste et continue de ralentir l'atteinte d'un développement équilibré entre les régions, ce n'est pas que les entrepreneurs des territoires périphériques soient incapables, timorés ou peu actifs. C'est plutôt que trop souvent l'ambiance économique et sociale se trouve encore alourdie d'un héritage sociohistorique qui n'encourage pas l'innovation. C'est le désintérêt chronique des pouvoirs publics, qui n'ont pas $\mathrm{vu}$, pour assurer l'occupation du territoire et son développement équilibré, la nécessité de mettre en place les infrastructures matérielles et didactiques dont les populations éloignées des grands centres rêvaient.

Ces dernières années, les entreprises des territoires périphériques ont acquis, grâce aux TIC, une liberté de manœuvre que diverses contraintes rendaient naguère impossible et qui de ce fait freinaient leur développement. L'assurance des entrepreneurs s'en trouve renforcée, de 
nouvelles possibilités apparaissent qui, si cette liberté s'accompagnait de conditions favorables, permettraient aux acteurs des territoires périphériques d'adopter des pratiques semblables à celles qui font la réussite des territoires métropolitains.

Pour les entreprises implantées en territoire périphérique, qui doivent faire face aux mêmes aléas que leurs semblables actives dans une métropole, l'adoption des outils technologiques appropriés et leur implication dans l'économie d'information sont les meilleurs moyens d'affronter la concurrence et de se faire une place dans le marché. Pour le territoire périphérique, la prospérité des entreprises qui y sont installées est l'une des conditions, peutêtre la plus importante, de son rattrapage et de sa propre prospérité.

Il est par ailleurs éminemment souhaitable que les acteurs d'accompagnement s'impliquent davantage en adaptant leurs interventions auprès des entreprises aux nouvelles façons de faire introduites par l'usage des TIC. De plus des efforts importants doivent venir de la part des pouvoirs publics. Il leur incombe de mettre en place les infrastructures nécessaires à l'entrée des économies périphériques dans l'ère de l'information.

S'il est essentiel, pour le rattrapage des territoires périphériques, qu'un minimum de conditions favorables soient réunies, il importe aussi, pour les chercheurs et les décideurs, qu'un monitorage factuel de l'évolution de la situation et des facteurs de progrès soit possible. L'étude des processus de développement et du rôle de l'innovation dans ces processus est à l'origine de théories, de concepts, dont le "système d'innovation" est l'un des plus récents avatars. Tour à tour sont apparus les systèmes: national, régional, puis spatial d'innovation, qui ont été passés en revue au chapitre 4 . Chacun de ces systèmes a ses avantages et son utilité; chacun a été conceptualisé pour répondre à un besoin particulier à un moment donné.

Pour cerner correctement les facteurs propices à linnovation et au développement dans un territoire, le choix du cadre spatial le plus approprié est difficile et délicat. Dans le cas des territoires de notre étude, l'observation, les données recueillies ainsi que les échanges de vues avec plusieurs acteurs locaux ont mené à la conclusion qu'aucun de ces concepts ne rendait compte, de façon satisfaisante, de la situation sur le terrain. Cest pourquoi il a paru utile de pousser plus loin la réflexion pour arriver à mieux refléter, à la fois le contexte actuel 
particulier de nos territoires périphériques au Québec, et limportance qu'y occupe désormais l'usage des technologies de pointe.

A cet égard, l'exemple de la région de Rivière-du-Loup illustre le pouvoir des nouvelles technologies pour activer l'économie d'un territoire. Cette région qui, lors de la cueillette (quand même récente) de nos données, trânait de l'arrière selon plusieurs indicateurs, s'est pour ainsi dire métamorphosée en guère plus de deux années, pour «devenir la capitale des PME à l'est de Québec».

S'il est vrai, comme nous sommes porté à le croire, que pour les territoires périphériques une ère de développement s'annonce, portée par les TIC et une apparente prise de conscience par les pouvoirs publics, le moment semble opportun pour la formulation d'un concept correspondant davantage aux nouvelles possibilités de nos régions. C'est pourquoi nous proposons une formule qui pourrait s'appeler "système périphérique d'action (ou d'innovation)".

Ce système serait un ensemble d'alliances et de réseaux, formé d'entreprises, d'organismes d'accompagnement, d'agences sociales et d'acteurs publics de divers paliers. Considérant la mondialisation croissante, linstantanéité de la communication et la contraction de la distance, le système ainsi créé serait à géométrie variable, les collaborations ou non se faisant selon les besoins et les contingences, mais la base opérationnelle demeurant à l'échelle d'un territoire donné. Il serait intéressant, croyons-nous, d'approfondir cette idée. 


\section{Conclusion}

On peut difficilement nier limportance du rôle des composantes technologiques, notamment des TIC, dans la vie quotidienne des individus et dans le fonctionnement des entreprises, Bien que d'apparition encore récente, le changement technologique dû à la numérisation et les outils qu'il a générés sont devenus pratiquement indispensables dans le monde des affaires et influencent de plus en plus la vie sociale.

Le présent travail avait pour but d'étudier comment l'appropriation et l'usage des TIC dans l'entreprise peuvent contribuer au développement régional. Selon notre analyse les TIC ne semblent pas avoir d'effets structurants démontrés sur le développement régional. Mais contrairement à l'historien, qui peut profiter d'un certain recul pour situer dans leur contexte et juger des événements qu'il analyse, le chercheur qui veut cemer la contribution présente des TIC au développement de l'économie d'un territoire se trouve devant un objet mouvant, dont l'évolution rapide est inhérente à la nature même de la technologie considérée.

Le matériel informatique, les logiciels et les objets qu'on peut y greffer changent constamment. Les connaissances et le savoir-faire qui servent à leur conception s'améliorent et se transforment, créant des objets plus complexes et performants. Même le vocabulaire n'y échappe pas et devient parfois source de confusion. Par exemple on utilise communément le vocable 'téléphone', oubliant que ce moyen de communication peut dériver d'une technologie autre que celle connue depuis un siècle. En effet la téléphonie IP (Internet Protocol), association du téléphone et d'Internet, permet de transmettre la voix de la même façon qu'on transporte les données. Le présent travail rapporte donc l'observation d'une situation à un moment donné - et les enseignements qu'on peut en tirer - une sorte d'instantané plutôt qu'une étude étalée dans le temps.

Ce travail conceme le développement régional et présente les résultats d'une étude réalisée dans quatre MRC : Rivière-du-Loup, Manicouagan, Saguenay et la CUQ. Ces territoires ont des vocations différentes; chacun a son propre parcours historique et social, et présente de 
nettes caractéristiques géographiques, économiques, politiques et culturelles. Si la CUQ est à la fois une métropole et une capitale où domine le secteur tertiaire, les MRC de Rivière-duLoup et de Manicouagan sont des territoires périphériques, où divers obstacles freinent le développement. On peut considérer le territoire de Saguenay comme étant dans une situation intermédiaire entre les deux premières catégories. Le secteur secondaire prédomine dans ces trois derniers territoires.

L'étude avait pour objet de connaître la pénétration des TIC dans les entreprises de ces territoires: appropriation, usage, effets, et d'établir des comparaisons entre les territoires. Afin de connaitre l'état des connaissances sur notre sujet de recherche, nous avons consulté une abondante littérature, tant nord-américaine qu'européenne. Cette revue nous a fait constater une nette évolution, depuis quelques décennies, dans la façon d'appréhender le développement régional; des opinions convergentes souvent, mais aussi divergentes, parmi les chercheurs.

Cet exercice nous a mené à formuler notre hypothèse relativement aux facteurs qui favorisent l'appropriation des TIC par les entreprises; l'usage qu'en font les entreprises pour la communication, l'information et la gestion; l'usage d'Internet et le commerce électronique; l'influence de la taille d'une firme sur l'utilisation des TIC et l'innovation, etc.

Comme le montrent les données descriptives, les territoires étudiés diffèrent sous plusieurs aspects: caractère urbain ou semi-rural, nombre d'entreprises, secteur principal d'emploi, etc. Les résultats de notre enquête dégagent cependant des tendances intéressantes chez les entreprises qui s'y trouvent: la modernité n'inquiète pas outre mesure et le changement technologique correspond généralement davantage aux besoins qu'à des contraintes de distance.

L'appropriation de la nouvelle technologie est estimée à l'aide des indicateurs suivants: l'usage de matériel informatique et de logiciels, le branchement et l'utilisation de l'Internet et du Web, et le soutien extérieur.

Dans le cas du matériel informatique, pratiquement toutes les entreprises de notre échantillon utilisent le télécopieur, lordinateur et l'imprimante. D'autres composantes plus spécialisées, telles que les robots, les lecteurs optiques, sont beaucoup moins présentes. Toutes les 
entreprises qui possèdent des ordinateurs possèdent au moins les logiciels de base: traitement de texte, comptabilité, etc. D'autres types de logiciels, bien que moins souvent mentionnés, parce que adaptés à des usages particuliers, sont aussi utilisés pour bases de données, gestion d'inventaire, gestion du personnel. Au moment de l'enquête, plus de la moitié des entreprises de l'échantillon étaient branchées à Internet depuis plus de deux ans.

On utilise l'Internet à des fins très variées, en premier lieu pour le courriel. Viennent ensuite l'acquisition de données stratégiques, les liaisons avec les fournisseurs, le repérage de nouveaux contacts, l'offre de produits et services, les sites vitrines, la communication interne, etc. C'est dans la région de Québec que l'Internet est le plus utilisé, par exemple pour la prise de commandes et l'ouverture sur le monde.

Le commerce électronique est assez peu développé. Seulement $32 \%$ des entreprises utilisent le Web pour faire des achats, la région de Québec arrivant en tête, suivie de Saguenay. Dans la plupart des entreprises, on est conscient que la Toile offre des possibilités qu'on pourrait exploiter bien davantage.

Pour s'adapter au changement technologique, les entreprises doivent assez souvent avoir recours à des compétences extérieures. Ce sont les conseillers et fournisseurs de matériels et de logiciels, dont les services sont le plus souvent requis par la majorité des firmes. Viennent ensuite les fournisseurs d'accès à Internet, les webmestres et les conseillers en communication.

Les sources d'information sont diversifiées. En général, les sources plus souvent utilisées sont les clients, les contacts personnels et les fournisseurs, les journaux et revues spécialisés. Les centres de recherche, les universités, les sociétés de développement et les services fédéraux sont rarement consultés.

Parmi les moyens d'interaction utilisés pour émettre ou recevoir l'information stratégique, le téléphone occupe la première place, suivi du télécopieur et du courriel. Par ailleurs les rencontres à l'extérieur de l'entreprise se font le plus souvent à l'occasion de repas, d'activités caritatives ou d'événements spéciaux. 
L'information recherchée est diversifiée. Elle concerne le plus souvent les nouveaux produits, la concurrence, les technologies et la gestion. Plus du tiers des entreprises ne semblent pas tenter de se renseigner sur les questions de sécurité, et autour de $20 \%$ sur les questions scientifiques ou juridiques.

Les réseaux inter-organisationnels ou réseaux d'entreprises sont généralement considérés comme d'importants lieux d'échange d'information et de concertation. Pourtant 6 entreprises sur 10 de notre échantillon n'y participent pas.

Pour leurs communications internes, les entreprises tablent essentiellement sur quatre systèmes. La réunion du personnel est de loin le moyen le plus courant. Suivent le tableau de nouvelles et le bulletin interne et d'intranet. Il y a lieu de noter qu'une entreprise peut utiliser à la fois plus d'un système et que les moyens choisis dépendent en bonne partie de sa taille.

On note aussi que l'usage du Web influe sur l'appropriation de l'informatique; que cette influence est particulièrement visible quand il s'agit de l'acquisition de données, de communication interne ou de gestion.

L'existence d'un système interne de communication est également favorable à l'appropriation de l'informatique, surtout l'usage d'un Intranet, qui amène la firme à se doter d'un équipement informatique performant. Par ailleurs la publication d'un bulletin interne influe sur la variété du matériel informatique mais moins sur la variété des logiciels.

Lorsqu'une entreprise sollicite un soutien extérieur, ce sont surtout les conseillers et fournisseurs d'équipement informatique qui sont appelés et ils influent davantage sur le choix du matériel que des logiciels. Les fournisseurs d'accès à Internet semblent avoir une influence plutôt négative.

Intuitivement, on présume que les activités extérieures, telles que les repas, les événements spéciaux, sont globalement bénéfiques pour l'entreprise. Pour ce qui est de l'appropriation et de l'usage des TIC toutefois, ces activités n'ont pas de signification statistique. De même l'affiliation à un réseau semble avoir moins d'effet que prévu; elle favorise cependant l'appropriation des logiciels. 
La taille d'une entreprise n'a pas d'effets très marquants sur son mode de fonctionnement; peu de différences attribuables à la taille sont significatives. On note que plus le nombre d'employés est important, moins la firme tend à diversifier ses sources d'information; mais que les moyens d'interaction (activités de face à face, de la place publique) y sont plus utilisés.

Une entreprise qui a plus d'une place d'affaires emploie moins le téléphone et le télécopieur, mais davantage I'Intranet. Quant aux moyens de communication interne, des variables telles que le bulletin interne, le tableau de nouvelles, les réunions du personnel, lorsque reliées à la taille de l'entreprise, ne sont pas significatives.

Les données brutes de notre étude montrent certains écarts entre les quatre territoires, quant à diverses variables considérées, par exemple l'appropriation et l'usage d'un équipement informatique, le branchement à l'Internet, etc.

L'ensemble de nos données trace un portrait nuancé des PME de quatre territoires et laisse entrevoir l'importance socio-économique qu'y occupent ces entreprises. Si une idée maitresse se dégage de notre étude, c'est sans doute l'emprise des TIC dans l'activité économique contemporaine. Nous avons souligné certains aspects très positifs, mais relevé aussi un retard assez généralisé quant à l'usage optimal des possibilités de linformatisation. Nous avons noté dans plusieurs cas la lenteur de l'appropriation des outils informatiques et l'usage relativement faible qu'on en fait. On ne semble pas se rendre suffisamment compte que les technologies nouvelles touchent désormais tous les aspects de la vie d'une entreprise; que les gains de productivité et la résistance à la concurrence en dépendent.

De façon générale, les entreprises auraient avantage à diversifier leurs sources d'information. Les collèges, les universités, les centres de recherche, sont des sources de savoir et d'innovation qu'on consulte trop peu. Les réseaux inter-organisationnels, les réseaux d'affaires, offrent des occasions de partage d'expérience et de savoir, et des lieux de concertation; une participation plus active et soutenue à ces organisations parait hautement souhaitable. Dans la plupart des entreprises, on ne semble pas se préoccuper suffisamment de sécurité: des données, des procédés, etc. De plus, les questions d'ordre scientifique et juridique ne suscitent pas beaucoup d'intérêt. 
Particulièrement dans les régions périphériques, il semble plus efficace de s'approprier une nouvelle technologie qui intègre en même temps les objets existants, plutôt que de s'en défaire d'un coup par un changement radical. Il n'y a pas lieu de chercher à remplacer les savoir-faire propres aux territoires périphériques, mais plutôt de les adapter aux exigences des nouvelles conjonctures, en misant sur les attitudes des acteurs et sur leur capacité à maintenir vivantes les normes et valeurs sociales propres à leur parcours socioculturel.

L'usage des TIC s'avère désormais indispensable au développement des régions, mais il ne s'accompagnera pas nécessairement de l'élimination de toute disparité entre territoires métropolitains et périphériques. L'objectif serait plutôt de permettre aux entreprises des territoires excentriques un rattrapage technologique satisfaisant, et aux régions, un développement économique et social qui assure aux populations un cadre de vie qui leur convienne et les incite à vouloir continuer à y habiter. À cet égard, une action des pouvoirs publics mieux coordonnée et plus soutenue s'avère indispensable.

Les employés d'entreprises et l'ensemble des citoyens peuvent également contribuer au développement en s'intéressant davantage au formidable monde de l'informatique. Particulièrement pour les usages personnels, l'équipement nécessaire est devenu plus convivial et l'initiation plus facile pour le nouvel utilisateur. L'usage personnel d'Internet, que l'on peut considérer au centre de l'innovation sociale, ouvre sur des horizons sans limites et amène souvent à s'approprier des composantes pouvant servir à toutes sortes d'autres fins. Encourager l'usage d'Internet dans les rapports sociaux est de nature à rendre un milieu favorable à l'innovation et au progrès. 


\section{Bibliographie}

- Acemoglu, Daron (2001), «Good Jobs versus Bad Jobs», Journal of Labor Economics, Vol. 19 , No. 1, pp. 1-21;

- Acs, Z. (2000), Regional Innovation, Knowledge and Global Change, London, Thomson Learning, $288 \mathrm{p}$.

- Acs, Zoltan J. et Audretsch David B. (1987), «Innovation, Market Structure, and Firm Size», Review of Economics and Statistics, 69, pp. 567-575;

- Acs, Zoltan J. et Audretsch David B. (1988), «Innovation in Large and Small Firms : An Empirical Analysis», American Economic Review, 78, pp. 678-690;

- Agre, Philip E. (2003), Information and Institutional Change: The case of digital libraries, to appear in Ann P. Bishop, Barbara P. Butterfield, and Nancy Van House, eds, Digital Library Use: Social Practice in Design and Evaluation, MIT Press, 341 p.

- Agre, Philip E. et Rotenberg, Marc (eds) (1997), Technology and Privacy: The New Landscape, Cambridge, MIT Press, 336 p.

- Agre, Philip E. et Schuler, Douglas (eds) (1997), Reinventing Technology, Rediscovering Community: Critical Explorations of Computing as a Social Practice, Greenwich, CT: Ablex, 272 p.

- Allen, Steven G. (2001), «Technology and the Wage Structure», Journal of Labor Economics, Vol. 19, No. 2. (Apr., 2001), pp. 440-483;

- Amable, Bruno, Barré, Rémi et Boyer, Robert (1997), Les systèmes d'innovation à l'ère de la globalisation, Paris, Économica, 401p.

- Amin, A., et Cohendet, P. (2000), «Organizational Learning and Governance through Embedded Practices», Journal of Management and Governance, Vol. 4 , pp. 93-116;

- Archibugi, D. et Pianta, M. (1992), The Technological Specialization of Advanced Countries; A report to the economic in international science and Technology Activities, Kluwer Academic Publishers, 184 p.

- Archibugi, Daniele, Howells, Jeremy et Michie, Jonathan (1999), Innovation Policy in Global Economy, Cambridge, England, Cambridge, University Press, $278 \mathrm{p}$.

- Asheim, Bjørn, T., and Philip Cooke (1999), «Local Learning and Interactive Innovation Networks in a Global Economy», pp. 145-178, in Malecki, Edward J., and Päivi Oinas (eds.). 1999, Making Connections. Technological 
Learning and Regional Economic Change. Ashgate Publishing Ltd., Aldershot Hants, England, 298 p.

- Augier, M., Cohen M., Dosi, G. et Levinthal, D. (2003), «Institutions and Organization : introduction to the special issue in honor of James G. March", Industrial and Corporate Change, Vol. 12, no 4, pp. 647-652.

- Aydalot, Philippe (1986), Milieux innovateurs en Europe = Innovative Environments in Europe, Paris, GREMI, 361 p.

- Aydalot, Philippe et David Keeble (1988), High Technology Industry and Innovative Environments : the European Experience, Routledge, London, 241 p.

- Bac, Mehmet (2000), «On-the-Job Specific Training and Efficient Screening», Journal of Labor Economics, Vol. 18, No. 4, pp. 681-701;

- Bailly S., Antoine (1992), "Représentation et analyse des territoires : une épistémologie de la science régionale", in, P.-H, Derycke, dir., Espace et dynamiques territoriales, Paris, Economica, 336 p.

- Bartel, Ann P. et Sicheman, Nachum (1993), «The Technological Change and Retirement Decisions of Older Workers», Journal of Labor Economics, Vol 11, (January 1993), pp. 162-183;

- Bartel, Ann P. et Sicheman, Nachum (1999), «The Technological Change and Wages: An Inter-Industry Analysis», Journal of Political Economy, vol. 107, (April 1999), pp. 285-325;

- Bartoli, Annie (1990), Communication et Organisation: pour une politique générale cohérente, Paris, Les éditions d'Organisation, 175 p.

- Benko, Georges et Lipietz, Alain (1992), Les Régions qui gagnent : districts et réseaux: les nouveaux paradigmes de la géographie économique, Paris, Presses universitaires de France, 424 p.

- Benko, Georges, Lipietz, Alain et al. (2000), La richesse des régions : la nouvelle géographie socio-économique, Paris, Presses universitaires de France, 564 pages;

- Blumenthal, Marjory S. (1998), «Federal Government Initiatives and the Foundations of the Information Technology Revolution: Lessons from History», The American Economic Review, Vol. 88, No. 2, Papers and Proceedings of the Hundred and Tenth Annual Meeting of the American Economic Association. (May, 1998), pp. 34-39;

- Blundell, Richard, Griffith, Rachel et Reenen, John Van (1999), «Market Share, Market Value and Innovation in a Panel of British Manufacturing Firms», The Review of Economic Studies, Vol. 66, No. 3, pp. 529-554. 
- Bontis, Nick et Choo, Chun Wei (2002), The Strategic Management of Intellectual Capital and Organizational Knowledge, Oxford University Press, New York;

- Borgman, Christine L. (2000), From Gutenberg to the Global Information Infrastructure. Access to Information in the Networked World, The MIT Press, Cambridge, $324 \mathrm{p}$.

- Borgman, Christine L. (2002), «Digital Libraries: People, Knowledge, and Technology: 5th International Conference on Asian Digital Libraries», Lecture Notes in Computer Science, Singapore Volume 2555, p. 1-13;

- Boschma, Ron (2004), «Proximité et innovation», Économie rurale, no 280 ;

- Bouchy, Serge (1994), L'ingénierie des systèmes d'information évolutifs, Paris, Eyrolles, $329 \mathrm{p}$.

- Bound, J., Cummins, C., Griliches, Z., Hall, B. H. et Jaffe, A. (1984), «Who does R\&D and Who Patents?», pp. 21-34; in, Z. Grilliches, (ed.), R\&D Patents and Productivity, Chicago, University of Chicago Press for the NBER, $512 \mathrm{p}$.

- Boyer, Robert (2001), «Le couple privé-public est fait pour durer», http://www.lemonde.fr/article/0,5987,3234-5411-229753,00.html (Consulté le 9 octobre 2001);

- Braczyk, Hans-Joachim, Cooke, Philip and Heidenreich, Martin (1998), Regional Innovation Systems: The Role of Governances in a Globalized World, London, UCL Press, 400 p.

- Breschi, S. Malerba, F. et Orsenigo, L. (2000), «Technological Regimes and Schumpeterian Patterns of Innovation», Economic Journal, vol. 110 (463), April 2000, pp. 388-410;

- Brousseau, Éric (c1993), L'économie des contrats : technologies de l'information et coordination interentreprises, Paris, Presses universitaires de France, $368 \mathrm{p}$.

- Caccomo, Jean-Louis (1996), Les défis économiques de l'information. La numérisation, Montréal et Paris, L'Harmattan inc. et Éditions L'Harmattan, $126 \mathrm{p}$.

- Callon, Michel (1991), «Réseaux techno-économiques et irréversibilités», pp. 195-230, in, Robert, Boyer, Chavance, Bernard et Godard, Olivier, Les figures de l'irréversibilité en économie, Paris, Éditions de l'École des hautes études en sciences sociales, 393 p.

- Camagni, Roberto (1991), Innovation Networks : Spatial Perspectives, Belhaven Press GREMI, London, 224 p. 
- Camagni, Roberto et Quévit, Michel (1992), Politique d'innovation technologique au niveau local -Innovation Policies at the Local Level, GREMI Università di Padova ;

- Castells, Manuel (1998), La société en réseaux : l'ère de l'information, (traduit de l'anglais par Philippe Delamare), Paris, Fayard, 671 p.

- Castells, Manuel (2002), La galaxie Internet, Paris, Fayard, 365 p.

- Chang, Wen-Ya, Tsai, Hsueh-Fang et Lai, Ching-Chong (1998), «Government Spending and Capital Accumulation with Endogenous Time Preference», The Canadian Journal of Economics, Vol. 31, No. 3. (Aug., 1998), pp. 624-645;

- Charbit, Claire, et Zimmermann, Jean Benoit (1998), "Systèmes d'information et entreprises : convergence ou incertitude ?», in terminal, technologie de l'information, Culture et Société, no 78 ;

- Choo, Chun Wei (1999), The Knowing Organization: How Organizations Use Information To Construct Meaning, Create Knowledge, and Make Decisions, Oxford University Press, New York, 320 p.

- Cohen, W. and Klepper, S. (1996b), «A Reprise of Size and R\&D», The Economics Journal, 106, 1996, pp. 925-951;

- Cohen, W. and Klepper, S., (1996a), «Firm Size and the Nature of Innovation with Industries: The Case of Process and Product R\&D, The Review of Economics and Statistics, 78, May 1996, pp. 323-243;

- Cohen, W. M., Levin, C. et Mowery, D.C. (1987), «Firm Size and R\&D Intensity: A Reexamination», Journal of Industrial Economics, vol. 35, no 4, pp. 543565

- Cohen, Wesley M. et Klepper, Steven (1996), "A Reprise of Size and R \& D», The Economic Journal, Vol. 106, No. 437. (Jul., 1996), pp. 925-951;

- Conti, Sergio (1993), «The Network Perspective in Industrial Geography: Towards a Model», Geografiska Annaler. Series B, Human Geography, Vol. 75, No. 3. (1993), pp. 115-130.

- Cooke, P. (2000), «Business Processes in Regional Innovation Systems in the European Union», pp.53-71, in, Acs, Z. (ed.), Regional Innovation, Knowledge and Global Change, London, Pinter, $224 \mathrm{p}$.

- Cooke, P. (2001), «From Technopoles to Regional Innovation Systems : The Evolution of Localised Technology Development Policy», Revue Canadienne des Sciences Régionales, V. XXIV, printemps 2001, pp. 21-40;

- Cooke, P. et Morgan, K. (1998), The Associational Economy: Firms, Regions, and Innovation, Oxford, Oxford University Press, $260 \mathrm{p}$. 
- Cooke, P., Bpekholt, P. et Todtling, F. (2000), The Governance of Innovation in Europe. Regional Perspectives on Global Competitiveness, London, Pinter, 192p.

- Cooke, P., M. Gomez Uranga et G. Extebarria (1997), Regional Innovation Systems: Institutional and Organizational Dimensions, Research Policy 26, pp. 475-491;

- Cornish, Susan L. (1997), "Product Innovation and the Spatial Dynamics of Market Intelligence: Does Proximity to Markets Matter?», Economic Geography, Vol. 73, No. 2. (Apr., 1997), pp. 143-165;

- Côté, Serge, Klein, Juan-Luis, et Proulx, Marc-Urbain (1995), Et les régions qui perdent-- ? : Actes du Colloque de la Section Développement régional de l'ACFAS 1994 tenu à l'UQAM les 17 et 18 mai 1994, Rimouski, Québec, GRIDEQ, 382 p.

- Couclelis, Helen (2004), «Pizza over the Internet: e-commerce, the Fragmentation of Activity and the Tyranny of the Region», Entrepreneurship \& Regional Development, Jan 2004, Vol. 16, no 1, pp. 41-54.

- Courlet, Claude (2001), Territoires et régions: les grands oubliés du développement économique, Paris, L'Harmattan, 133 pages.

- Crevoisier, Olivier et Camagni, Roberto, éds (2000), Les milieux urbains: innovation, systèmes de production et ancrage, IRER, EDES, Neuchâtel ;

- de LA Mothe, John et Paquet, Gilles (1998), Local and Regional Systems of Innovation, Economics of Science, Technology, and Innovation, Vol 14), $360 \mathrm{p}$.

- de la Mothe, John et Paquet, Gilles (2000), Information, Innovation and Impacts, Economics of Science, Technology and Innovation, Vol. 17, Norwell, Mass., Kluwer Academic, 360 p.

- de Terssac, G. et Tremblay, D.-G. (2000), Où va le temps de travail, Toulouse, Octarès Editions, 284 p.

- Dosi, G., Levinthal, D. A. et Marengo, L. (2003), «Bridging Contested Terrain : linking incentive-based and learning perspectives on organizational evolution», Industrial and Corporate Change, Vol. 12, no 2, pp. 413436.

- osi, G., Malerba, F. et Teece, D. (2003), «Twenty Years after Nelson and Winter's. An Evolutionary Theory of Economic Change: a preface on knowledge, the nature of organizational changes», Industrial and Corporate Change, Vol. 12, no 2, pp. 147-148.

- Dosi, Giovanni (1982), «Technical Paradigms and Technological Trajectories: a Suggested Interpretation of the Determinants and Direction of Technical Change», Research Policy, vol. 11, pp. 147-162; 
- Dosi, Giovanni (1997), «Opportunities, Incentives and the Collective Patterns of Technological Change», The Economic Journal, Vol. 107, No. 444. (Sep. 1997), pp. 1530-1547;

- Dupuy, Claude et Filippi, Maryline (2000), «Marchés, organisation, territoires et confiance: le cas des réseaux de producteurs dans le domaine alimentaire», Revue d'Économie Régionale et Urbaine, no 3, pp. 519-534.

- Durkheim, E., (1985), Les formes élémentaires de la vie religieuse: le système totémique en Australie, Paris, Alcan, 198, ( $7^{\mathrm{e}}$ édi.), (1912), 647 pages.

- Edquist, C. (Ed.) (1997), Systems of Innovation, Technologies, Institutions and Organizations, London et Washington, Pinter, $320 \mathrm{p}$.

- Edquist, C. et Lundvall, B.-A. (1993) «Comparing the Danish and Swedish Systems of Innovations», In: Nelson, R.R. (Ed.), National Innovation Systems, Oxford University Press, New York, 560 p.

- Evans, Philip et Wurster, Thomas S. (2000), Blown to Bits : how the new economics of information transforms strategy, Boston, Mass., Harvard Business School Press, 261 p.

- Florida, Richard (2000), «La destruction créatrice à l'échelle régionale : l'organisation de la production, la mondialisation et les transformations du Midwest», pp. 359-389; in, Benko, Georges et Lipietz, Alain, (dir.), (2000) La richesse des régions : la nouvelle géographie socio-économique, Paris, Presses universitaires de France, $564 \mathrm{p}$.

- Foray, D. et Steinmueller,E (2003), «The Economics of Knowledge Reproduction by inscription», Industrial and Corporate Change, vol. 12, no 2, pp. 299319.

- Foray, Dominique et Freeman, Christopher (1992), Technologie et richesse des nations, Économica, Paris, $517 \mathrm{p}$.

- Fortin, A. et Sanderson, D. (2004), Espaces et identités en construction. Le Web et les régions du Québec, Québec, Nota Bene, 157 pages.

- Godin, B., Archambault, E. et Vallières, F. (2000), «La production scientifique québécoise : mesure basée sur la BRSQ, Argus, Vol., 29, no 1, pp. 1523.

- Gosselin, Maurice et Simon, Samuel (2000), De meilleurs services aux entreprises grâce aux nouvelles technologies : des outils d'aide à la décision, Montréal, CEFRIO, $97 \mathrm{p}$.

- Granovette, Mark (1994), «La construction économique des institutions», in, Orléan, André, Analyse économique des conventions, Paris, PUF, 403 p. 
- Greenaway, David (1994), «Policy Forum: The Diffusion of New Technology: Editorial Note», The Economic Journal, Vol. 104, No. 425. (Jul., 1994), pp. 916-917;

- Habermas, Jürgen (1987), Théorie de l'agir communicationnel, traduit de l'allemand par Jean-Marc Ferry, Paris, Fayard ;

- Hamberg, Daniel (1964) «Size of Firm, Oligopoly and Research: The Evidence», Canadian Journal of Economics and Political Science, Vol. 30, pp. 62-75;

- Harrison, Bennett, Kelley, Maryellen R. et Gant, Jon (1996), Innovative Firm Behavior and Local Milieu: Exploring the Intersection of Agglomeration, Firm Effects, and Technological Change, Economic Geography, Vol. 72, No. 3. (Jul., 1996), pp. 233-258;

- Holemans, B., et L. Sleuwaegen (1988), «Innovation Expenditures and the Role of Government in Belgium), Research Policy, 17, pp. 375-379;

- Isaksen, A. (2001), «Building Regional Innovation Systems: Is Endogenous Industrial Development Possible in the Global Economy?», Revue canadienne des sciences régionales, XXIV, 1, pp. 101-120;

- Jones, Robert et Newman, Geoffrey (1995), «Adaptive Capital, Information Depreciation and Schumpeterian Growth», The Economic Journal, Vol. 105, No. 431, pp. 897-915;

- Jorgenson, Dale W. et Stiroh, Kevin J. (1999), «Information Technology and Growth» The American Economic Review, Vol. 89, No. 2, Papers and Proceedings of the One Hundred Eleventh Annual Meeting of the American Economic Association, pp. 109-115;

- Julien, P.-A. (2001), «Le modèle québécois simplifié et vilipendé de nouveau par les ultralibéraux», Le Devoir, 28 mars 2001, p. A6.

- Julien, Pierre-André (1996) «Entrepreneuriat, développement régional et appropriation de l'information», dans Proulx, Marc-Urbain, (dir.), Le phénomène régional au Québec, Québec, Presses de l'Université du Québec, $317 \mathrm{p}$.

- Kahneman, D., and Frederick, S. (2002), «Representativeness Revisited: Attribute Substitution in Intuitive Judgment», pp. 49-81, In T. Gilovich, D. Griffin and D. Khneman (Eds.) (2002), Heuristics and Biases: The Psychology of Intuitive Judgment, New York, Cambridge University Press, $874 \mathrm{p}$.

- Klepper; S. et Simons K. L. (2000), «The Making of an Oligopoly: Firm Survival and Technological Change in the Evolution of the U.S. Tire Industry", The Journal of Political Economy, Vol. 108, No. 4. (Aug., 2000), pp. 728-760; 
- Krueger, Alan B.. (1993), «How Computers Have Changed the Wage Structure : Evidence from Microdata, 1984-1989», Quarterly Journal of Economics, Vol 108, (February 1993), pp. 33-60;

- Lagacé, M. et Prévost, P. (2003), «Démocratie, e-démocratie et gouvernance locale : réflxion sur les nouvelles voies de solution aux enjeux actuels de développement collectify, Rencontres internationales : Démocratie et Management local, 15 pages.

- Lall, S., (1999), "Technological Change in Industrialization in the Asian NIEs: Achievements and Challenges», pp. 13-68, in: A. Szirmai, J. Halman and B. Verspagen (eds), Innovation in Theory and Practice, Eindhoven University of Technology;

- Landry, Réjean, Amara, Nabil et Lamari, Moktar (2001), «Capital social, innovation et politiques publiques», ISUMA, Canadian Journal of Policy Research/Revue canadiemne de recherche sur les politiques, Vol. 2 no 1, pp. 63-71;

- Landry, Réjean, Lamari, Moktar et Nimijean, Richard (1999), «Stimuler l'innovation par le développement de milieux créateurs : un examen des politiques et pratiques émergentes, réseaux du Québec pour la promotion des systèmes d'innovation (RQSI)», Rapport de veille présenté à l'Observatoire de développement économique du Canada, DÉC, Montréal, $88 \mathrm{p}$.

- Laramée, A. (2001), La communication dans les organisations : une introduction théorique et pragmatique, Sainte-Foy, Télé-université, $266 \mathrm{p}$.

- Laramée, A. et Vallée, B (1991), La recherche en communication: éléments de méthodologie, Presses de l'Université du Québec, Sainte-Foy, 377 p.

- Lehr, Bill et Lichtenberg, Frank (1999), «Information Technology and Its Impact on Productivity: Firm-Level Evidence from Government and Private Data Sources, 1977-1993», The Canadian Journal of Economics, Vol. 32, No. 2, Special Issue on Service Sector Productivity and the Productivity Paradox, pp. 335-362;

- Lévy, Pierre (1992), De la programmation considérée comme un des beaux-arts, Paris, La Découverte, 245 pages.

- Licht, Georg et Moch, Dietmar (1999), «Innovation and Information Technology in Services», The Canadian Journal of Economics, Vol. 32, No. 2, Special Issue on Service Sector Productivity and the Productivity Paradox, pp. 363-383;

- Link, Albert N. (1982), Firm Size and Efficient Entrepreneurial Activity: A Reformulation of the Schumpeter Hypothesis», Journal of Political Economy, 88: 41, pp. 771-782; 
- Lovallo, D., and Kahneman, D. (2000), «Living with Uncertainty: Attractiveness and Resolution Timing», Journal of Behavioral Decision Making, no 13, pp. 179-190.

- Lundvall, B. - A. (ed.) (1992), National Systems of Innovation: Towards a Theory of Innovation and Interactive Learning, London, Pinter Publishers, 556 p.

- Lundvall, B.-A. (1992a), "Relations entre utilisateurs et producteurs, systèmes nationaux d'innovation et internationalisation", pp. $355-388$, in Foray, D. et Freeman, C., (eds.), Technologie et richesse des nations, Economica, Paris, $517 \mathrm{p}$.

- Lundvall, B.-A. (2004), «Introduction to 'Technological Infrastructure and International Competitiveness' by Chrisopher Freeman", Industrial and Corporation Change, Vol. 13, no 3, pp. 531-539.

- Lundvall, B.-A. and Johnson, B. (1994), 'The Learning Economy', Journal of Industry Studies, vol. 1. no.2, December 1994, pp. 23-42;

- Lundvall, B-A. (1988) «Innovation as an Interactive Process: from User-product Interaction to the National System of Innovation», pp. 349-69, in: Dosi, G., Freeman, C., Nelson, R. Silverberg, G. et Soete, L., Technical Change and Economic Theory, London, Frances Pinter, $656 \mathrm{p}$.

- Maillat, Denis et Perrin, Jean-Claude (1992), Entreprises innovatrices et développement territorial, GREMI-EDES, Neuchâtel, 260 p.

- Maillat, Denis, Quévit, Michel et Senn, Lanfranco (1993), Réseaux d'innovation et milieux innovateurs : un pari pour le développement régional, GREMI-EDES, Neuchâtel, 388 p.

- Malecki, E. et al. (2004), «Entrepreneurship and Space in the Network Age», Editorial, Entrepreneurship \& Regional Development, Jan. 2004, Vol. 16, nol, pp. 1-3.

- Malecki, E. et al. (2004a), «Fibre Tracks: explaining investment in fibre optic backbones», Entrepreneurship \& Regional Development, Jan. 2004, Vol. 16, no1, pp. 21-39.

- Malecki, E. J. (1991), Technology and Economic Development : the dynamics of local, regional, and national change, Harlow, Longman Scientific \& Technical; New York : J. Wiley \& Sons, 495 p.

- Malecki, E. J. (1998), Entrepreneurs, Networks, and Economic Development: a review of recent research, pp. 57-118, in J.A. Katz (ed.) Advances in Entrepreneurship, Firm Emergence and Growth, vol. 3. Greenwich, CT: JAl Press, $250 \mathrm{p}$.

- Malecki, E. J. (2000) «Creating and Sustaining Competitiveness: local knowledge and economic geography», pp. 103-119, in P. Daniels, J. Bryson, N. 
Henry, and J. Pollard (eds.) Knowledge, Space, Economy, London, Routledge, $328 \mathrm{p}$.

- Malecki, E. J. er Gormain, S. P. (2001), «Maybe the Death of Distance, But Not the End of Geography: The Internet as a Network», pp. 87-105, In Leinbach, T. R. et Brumn, S. D., eds., (2001), World of Electronic Commerce: Economic, Geographical and Social Dimensions, New York, John Wiley, $372 \mathrm{p}$.

- Malecki, E. J. et Veldhoen, M. E. (1993), «Network Activities, Information and Competitiveness in Small Firms», Geografiska Annaler, Series B, Human Geography, Vol. 75, No. 3. (1993), pp. 131-147;

- Malecki, E.J. et Oinas, Päivi (1999), "Spatial Innovation Systems», in Malecki, Edward J., and Päivi Oinas (eds.) (1999), Making Connections. Technological Learning and Regional Economic Change. Ashgate Publishing Ltd. Aldershot Hants, England, 298 p.

- Malecki, Edward J., Oinas, Päivi et Ock Park, Sam (1999), «On Technology and Development» in Malecki, Edward J., and Päivi Oinas (eds), (1999) Making Connections, Technological Learning and Regional Economic Change, Ashgate Publishing Ltd. Aldershot Hants, England, 298 p.

- Malerba, F. et Orsenigo, L. (1995), «Schumpeterian Patterns of Innovation Country Specific, Technology Specific, or Both?», Cambridge Journal of Economics, vol. 19 (1), February 1995, pp. 47-65;

- Malmberg, Anders (1996), Industrial Geography: Agglomeration and Local Milieu. In: Progress in Human Geography, 20(3), pp. 392-403.

- Malmberg, Anders (1997), Industrial Geography: Location and Learning. In: Progress in Human Geography, 21(4), pp. 573-582.

- Malmberg, Anders et Maskell, Peter (1999), «Localised Learning and Industrial Competitiveness) In: Cambridge Journal of Economics, 23, pp. 167185.

- Malmberg, Anders; Maskell, Peter (1999), "The Competitiveness of Firms and Regions» In: European Urban and Regional Studies, 1(6), pp. 9-25.

- Malmberg, Anders; Svlvell, Vrjan; Zander, Ivo (1996), «Spatial Clustering, Local Accumulation of Knowledge and Firm Competitiveness», In: Geografiska Annaler, 78 B (2).(1996), pp. 85-97.

- Marx, Karl (c1966), Lidéologie allemande, traduction de Renée Cartelle et Gilbert Badia, Paris, Editions sociales, (col. Classiques du marxisme), 150 p.

- Mayère, Anne (1990), Pour une économie de l'information, Paris, Édition du CNRS, 316 p. 
- Mayère, Anne (1997), La société informationnelle : enjeux sociaux et approches économiques, Paris et Montréal, Éditions L'Harmattan et L'Harmattan inc., 239 p.

- Metcalfe, S. (1995), «The Economic Foundations of Technology Policy», in : Stoneman, P. (ed), Handbook of the Economics of Innovation and Technical Change, Oxford, Blackwell, $583 \mathrm{p}$.

- Miguelez, Roberto, Les règles de l'interaction : essai en philosophie sociologique, Québec et Paris, Collection Zêtésis, Les Presses de l'Université Laval et L'Harmattan, 2001, 344 p.

- Mitchell, William J. Inouye, Alan S. et Blumenthal, Marjory S. (2003), Beyond Productivity: Information, Technology, Innovation, and Creativity, Washington, DC, The National Academies Press, 268 pages.

- Nelson, R. R. et Rosenberg, N. (1993), «Technical Innovation and National Systems», In Nelson, R.R. (Ed.), National Innovation Systems: A comparative Analysis, Oxford University, Press, New York, $556 \mathrm{p}$.

- Nelson, Richard R. et Winter, Sidney G. (1982), An Evolutionary Theory of Technical Change, Cambridge, MA, Harvard University Press, $437 \mathrm{p}$.

- Nelson, Richard R. et Wright, Gavin (1992), «The Rise and Fall of American Technological Leadership: The Postwar Era in Historical Perspective», Journal of Economic Literature, Vol. 30, No. 4. (Dec., 1992), pp. 1931-1964.

- Niosi, J. (2002), «National Systems of Innovation are " $x$-efficient" (and $x$-effective) Why Some are Slow Learners "), Research Policy 31 (2002), pp. 291-302;

- Niosi, J., Saviotti, P.P., Bellon, B. et Crow, M. (1993), National Systems of Innovation: in Search of a Workable Concept, Technology in Society 15, pp. 207227 ;

- Niosi, J. (2001), "Alliances are not enough Explaining rapid Growth in Biotechnology Firms» Research Policy, 32 (2003), 737-750; http://www.er.uqam.ca/nobel/r21010/document/alliances\%20are\%20n ot $\% 20$ enough.pdf

- Nooteboom, Bart (2000), «Learning by Interaction: Absorptive Capacity, Cognitive Distance and Governance», Journal of Management and Governance, Vol. 4 , pp. 69-92.

- OCDE (1999), Tableau de bord de l'OCDE de la science, de la technologie et de l'industrie 1999: mesurer les économies fondées sur le savoir, Division de l'Analyse économique et de statistiques (AES) de la Direction de la science, de la technologie et de l'industrie (DSTI) Paris, $178 \mathrm{p}$. 
- OCDE (2000b), Société du savoir et gestion des connaissances, OCDE, Centre pour la recherche et l'innovation dans l'enseignement, (Col. : Enseignement et compétences) Paris, OCDE, $288 \mathrm{p}$.

- OCDE (2001), Perspectives territoriales de l'OCDE. Économie territoriale, OCDE, 310 p.

- OCDE (2001), Mesurer la productivité. Manuel de l'OCDE. Mesurer la croissance de la productivité par secteur et pour l'ensemble de l'économie, Publications de l'OCDE, p. 162.

- OCDE (2004), «Promouvoir l'entrepreneuriat et les PME innovantes dans une économie mondiale: Vers une mondialisation plus responsable et mieux partagée», $2^{\mathrm{e}}$ Conférence de l'OCDE des ministres en charge des petites et moyennes entreprises (PME), Istanbul, Turquie, 3-5 juin 2004, 53 pages.

- Oinas, P. (1999), «Activity-specificity in Organizational Learning : Implications for Analyzing the Role of Proximity», GeoJournal, 49, pp. 363-372;

- Oinas, P. (2000), «Distance and Learning: Does Proximity Matter ?», In: Rutten, R., Bakkers, S., Morgan, K. et Boekema, F. (2000), Knowledge, Innovation and Economic Growth: Theory and Practice of the Learning Region, Cheltenham, UK, Edward Elgar, 288 p.

- Oinas, P. et Malecki, E. J. (2002), «The Evolution of Technologies in Time and Space: From National and Regional to Spatial Innovation Systems», International Regional Science Review, Vol., 25, no 1, pp. 102-131;

- Pavitt, K. (1996), National Policy for Technical Change : Where are the Increasing Returns to Economic Research ? (http://www.pubmedcentral.gov/articlerender.fcgi?tool=pmcentrez\&ar tid=34124, consulté le 20 décembre 2003).

- Pavitt, K., M. Robenson et J. Townsend (1987), «The Size of Innovating Firms in the UK : 1945 - 1983», Journal of Industrial Economics, 35, pp. 297-316;

- Perroux F. (1935), La pensée économique de Joseph Schumpeter ; introduction à La théorie de l'évolution économique. Recherches sur le profit, le crédit, l'intérêt et le cycle de la conjoncture. J. Schumpeter, Dalloz, 1935, (traduction de la seconde édition en anglais 1926$), 589 \mathrm{p}$.

- Petit, Pascal (1998), L'économie de l'information : les enseignements des théories économiques, Paris, La Découverte, $405 \mathrm{p}$.

- Piveteau, Jean-Luc (1995), Temps du territoire : continuité et rupture de la relation de l'homme et l'espace, Carouge-Genève, Éditions Zoé ;

- Planque, B. (1991), «Note sur la notion de réseau d'innovation», Revue d'Économie Régionale et Urbaine, no 3-4, pp. 295-320. 
- Polèse, Mario et Shearmur, R., (et al.) (2002), La périphérie face à l'économie du savoir. La dynamique spatiale de l'économie canadienne et l'avenir des régions non métropolitaines du Québec et des provinces de l'Atlantique, Montréal, INRS Urbanisation, Culture et Société et I'Institut canadien de recherche sur le développement régional, $237 \mathrm{p}$.

- Porter, M. (1993), L'avantage concurrentiel des Nations, (traduit de l'américain par Pierre Miraillès, Catherine Barthélémy et Eve Dayre-Mielcarski), SaintLaurent, Éditions du Renouveau pédagogique ; Paris, Inter-éditions, 883 p.

- Porter, M. (1999), La concurrence selon Porter, Paris, Village mondial, 475 p.

- Porter, M. (2003a), Regional Competitiveness: Towards an Agenda for London, (20 juin 2003) http://www.one-london.com/News/MPorterCommentary.pdf : consulté le 20 juillet 2003)

- Porter, M. (2003b), «The Competitive Advantage of South Africa», Johannesburg, South Africa, (9 juin 2003), http://www.isc.hbs.edu/pdf/CAON_South\%20Africa_2003.06.09_CK .pdf : consulté le 20 juillet 2003)

- Prévost, P. (2002), «Les dimensions et processus du développement des collectivités locales», Cahier de recherche IREC 01-05, IRECUS, Université de Sherbrooke, 32 pages.

- Proulx, Marc-Urbain (1995), Réseaux d'information et dynamique locale, Chicoutimi, GRIR, Université du Québec à Chicoutimi ;

- Proulx, Marc-Urbain (1998), L'organisation des territoires au Québec, Tiré à part, L'Action Nationale, février-mars;

- Proulx, Marc-Urbain (2002), «Bilan de la politique des pôles de croissance. Relancer l'idéal d'un Québec polycentrique», in, Roch, Côté, (dir.),. Annuaire politique, social, économique et culturel, FEDES, Québec;

- Proulx, Marc-Urbain et Mévellic, Anne (2002), "La longue marche de la consolidation au Saguenay", in, Roch, Côté, (dir.), Québec 2002. Annuaire politique, social, économique et culturel, FEDES, Québec, p. 357-358.

- Proulx, Marc-Urbain, (dir.) (1996), Le phénomène régional au Québec, Québec, Presses de l'Université du Québec ;

- Proulx, Marc-Urbain, et Riverin, Nathalie (1998), "Dynamiques spatiales de l'entrepreneuriat: le cas du Québec", Communication présentée au XXXIVe colloque annuel de l'ASRDLF, villes, régions et développement, tenu à Benemérita Universidad Autonoma de Puebla, Mexique, septembre ; 
- Proux, Marc-Urbain (1995), "Les petits milieux innovateurs", in l'Action Nationale, Vol, LXXXV, numéro 7 ;

- Proux, Marc-Urbain (1995), «Trois échelons territoriaux» in l'Action Nationale;

- Rallet, A. (2000), "Les deux économies de l'information", Réseaux, no 100;

- Rallet, A. et Torre, A. (2004), «Proximité et localisation», Économie rurale, no 280 ;

- Rammert, Werner (1997), New Rules of Sociological Method: Rethinking Technology Studies, The British Journal of Sociology, Vol. 48, No. 2. (Jun., 1997), pp. 171-191;

- Ratti, R. (1992), Innovation technologique et développement régional : base théorique et étude de cas, Bellinzona, Lausanne, Méta-Editions, $148 \mathrm{p}$.

- Ratti, Remigio, Gordon R. et Bramanti, A. (1997), The Dynamics of Innovative Regions : The GREMI Approach, Aldershot, Ashgate, 391 p.

- Robillard, Jean (2004), Communications électroniques et dynamique organisationnelle, Presses de l'Université du Québec, (Communication organisationnelle), 304 pages.

- Ruttan, Vernon W. (1997), «Induced Innovation, Evolutionary Theory and Path Dependence: sources of technical change», The Economic Journal, Vol. 107 , No. 444, pp. 1520-1529;

- Ruttan, Vernon W. (2000), Technology, Growth and Development: An Induced Innovation Perspective, Oxford University Press Inc., 672 pages.

- Ruttan, Vernon W. (2003), Social Science Knowledge and Economic Development. An Institutional Design Perspective, University of Michigan Press, 364 pages.

- Salais, Robert (1994), «Incertitude et interactions de travail : des produits aux conventions», pp.371-403, in, Orléan, A., Analyse économique des conventions, Paris, PUF, $403 \mathrm{p}$.

- Scherer, F. M. et Ross, D. (1990), «Industrial Market Structure and Economic Performance», Boston, Houghton Mifflin Company, 713 p.

- Schumpeter, J.-A. (1935, c 1912), Théorie de l'évolution économique. Recherches sur le profit, le crédit, lintérêt et le cycle de la conjoncture, Dalloz, $589 \mathrm{p}$.

- Schumpeter, J.-A. (1939), Business Cycles, a Theorical, Historical and Statistical Analysis of The Capitalist Process, New-York, London, Mcgraw Hill Book Company, 448 p.

- Schumpeter, J.-A. (1979, c1947), Capitalisme, socialisme et démocratie, (traduit de l'anglais par Gaël Fain), Paris, Payot, (1942), 1979, 417 pages. 
- Sfez, L. (1999), «L'idéologie des nouvelles technologies», Révolution dans la communication, Paris, Le monde diplomatique, 98p.

- Shearmur, R. (2004a), «Migration des emplois en SFTU : ce que Montréal peut apprendre du passé», Recherche effectuée dans le cadre de la conférence Migration des emplois en TI vers des économies émergentes: avantages et défis pour le Québec, Organisée par TECHNOCompétences.

- Sicard, Marie-Noël et Mesnier, Jean-Michel, (dir) (1998), Les Technologies de IInformation et de la Communication : pour quelle société? : actes du colloque, 28, 29 et 30 avril 1997, Compiègne [Oise], Université de Technologie de Compiègne, $271 \mathrm{p}$.

- Simmie, James (1997), Innovation, Networks and Learning Regions ?, London, Bristol, PA, Jessica Kingsley; London, Regional Studies Association, 250 p.

- Singh, Kulwant (1997), "The Impact of Technological Complexity and Inter-firm Cooperation on Business Survival», The Academy of Management Journal, Vol. 40, No. 2, Special Research Forum on Alliances and Networks, pp. 339-367;

- Sole, Deborah et Edmondson, Amy (2002), «Bridging Knowledge Gaps: Learning in Geographically Dispersed Cross-functional Development Teams", In: Bontis, Nick et Choo, Chun Wei (2002), The Strategic Management of Intellectual Capital and Organizational Knowledge, Oxford University Press, New York, 768p.

- Stein, Jeremy C. (1997), Waves of Creative Destruction: Firm-Specific Learning-by-Doing and the Dynamics of Innovation, The Review of Economic Studies, Vol. 64, No. 2. pp. 265-288.

- Stoneman, P. (1995), Handbook of the Economics of Innovation and Technological Change, Oxford et Cambridge, Blackwell, 1995, 583p.

- Storper, M. (1996), «Innovation as Collective Action : Conventions, Products and Technologies», Industrial and Corporate change, Vol. 5, 1996, pp. 761-790;

- Storper, Michael (1999), «Technologie, stratégies des firmes et ordre territorial», Sciences de la Société, no 48, Presses Universitaires de Mirail;

- Storper, Michael (c1997), The Regional World : Territorial Development in a Global Economy, New York, Guilford Press, 338 p.

- Storper, Michael et Salais, Robert (1993), Les mondes de production : enquête sur l'identité économique de la France, Cambridge, Mass., Harvard University Press, $467 \mathrm{p}$. 
- Triplett, Jack E. (1999), «The Slow Productivity Paradox: What do Computers do to Productivity?», The Canadian Journal of Economics, Vol. 32, No. 2, Special Issue on Service Sector Productivity and the Productivity Paradox. (Apr., 1999), pp. 309-334;

- Villard, Henry, H. (1958), "Competition, Oligopoly and Research», Joumal Of Political Economy, 1958, volume 66, pp. 483-497;

- Wolf, Edward N. (1999), «The Productivity Paradox: Evidence from Indirect Indicators of Service Sector Productivity Growth», The Canadian Journal of Economics, Vol. 32, No. 2, Special Issue on Service Sector Productivity and the Productivity Paradox. (Apr., 1999), pp. 281-308;

- Worley, J. S. (1961), «Industrial Research and New Competition», Journal Of Political Economy, volume 69, pp. 183-186;

- Wright, Gavin (1997), «Towards a More Historical Approach to Technological Change», The Economic Journal, Vol. 107, No. 444, pp. 1560-1566;

- Zimmermann, Jean-Benoit (1995), «L'industrie du logiciel : de la protection à la normalisation», pp. 195-207, in, Basle, Maurice et al., Changement institutionnel et changement technologique : évaluation, droits de propriété intellectuelle, système national d'innovation, CNRS Édition, $333 \mathrm{p}$. 